\title{
Base Induced Chiral Substituted Furans and Imidazoles from
}

\section{Carbohydrate-Derived 2-Haloenones}

\author{
Kanchan Mal and Indrajit Das* \\ Organic and Medicinal Chemistry Division, CSIR-Indian Institute of Chemical Biology, 4, \\ Raja S. C. Mullick Road, Jadavpur, Kolkata 700 032, India. E-mail: id@ csiriicb.in
}

\section{Table of Contents:}

1. X-ray Crystal Structure. $\mathrm{S} 2-\mathrm{S} 3$

2. Copy of $1 \mathrm{D}$ and $2 \mathrm{D}$ NMR Spectra. S4-S118 


\section{CRTYSTAL STRUCTURE}

Single crystals were grown by slow evaporation of the compounds from acetone solvent.

Data were collected at $293 \mathrm{~K}$ on a X-ray diffractometer. Preliminary lattice parameters and orientation matrices were obtained from three sets of frames. Then full data were collected using the $\omega$ and $\phi$ scan method with the frame width of $0.5^{\circ}$. Multiscan absorption corrections were applied by using the SADABS program for area detector. ${ }^{1}$ All structures were solved with SHELXT ${ }^{2}$ and refined with SHELXL $^{3}$ using Olex2 program. ${ }^{4}$

References:

(1) Sheldrick, G. M. SADABS, Program for empirical absorption correction of area-detector data; Universität Göttingen: Göttingen, Germany, 2008. (2) Sheldrick, G. M. Acta Cryst., 2015, A71, 3-8. (3) Sheldrick, G. M. Acta Cryst. 2015, C71, 3-8. (4) Dolomanov, O. V.; Bourhis, L. J.; Gildea, R. J.; Howard, J. A. K.; Puschmann, H. J. Appl. Cryst. 2009, 42, 339341

\section{ORTEP diagram}


Figure S1. X-ray determined molecular structure of 5a. The thermal ellipsoids are shown in 50\% probability level. (CCDC 1430657)

\section{Datablock: 5a}

\begin{tabular}{llll}
\hline Bond precision: $\quad \mathrm{C}-\mathrm{C}=0.0019 \mathrm{~A}$ & Wavelength=0.71073 \\
Cell: & $\mathrm{a}=9.0301(11) \quad \mathrm{b}=9.6899(11)$ & $\mathrm{C}=11.5773(14)$ \\
$\mathrm{alpha}=89.237(5)$ beta=88.378(6) & gamma=70.942(4) \\
Temperature: $293 \mathrm{~K}$ &
\end{tabular}




\begin{tabular}{|c|c|c|}
\hline & Calculated & Reported \\
\hline Volume & $957.1(2)$ & $957.1(2)$ \\
\hline Space group & $P-1$ & $P-1$ \\
\hline Hall group & $\begin{array}{ll}-\mathrm{P} & 1\end{array}$ & -P 1 \\
\hline Moiety formula & C23 H20 O5 & C23 H20 O5 \\
\hline Sum formula & C23 H20 O5 & C23 H20 O5 \\
\hline $\mathrm{Mr}$ & 376.39 & 376.39 \\
\hline $\mathrm{Dx}, \mathrm{g} \mathrm{cm}-3$ & 1.306 & 1.306 \\
\hline Z & 2 & 2 \\
\hline $\mathrm{Mu} \quad(\mathrm{mm}-1)$ & 0.092 & 0.092 \\
\hline F000 & 396.0 & 396.0 \\
\hline F000' & 396.21 & \\
\hline $\mathrm{h}, \mathrm{k}, \mathrm{lmax}$ & $11,12,15$ & $11,12,15$ \\
\hline Nref & 4442 & 4422 \\
\hline Tmin, Tmax & $0.973,0.982$ & $0.694,0.746$ \\
\hline Tmin' & 0.973 & \\
\hline $\begin{array}{l}\text { Correction method= } \\
\text { Tmax }=0.746 \text { AbsCorr }\end{array}$ & $\begin{array}{l}\text { \# Reported T Limits: Tmin=0.694 } \\
=\text { MULTI-SCAN }\end{array}$ & \\
\hline Data completeness $=$ & $0.995 \quad$ Theta $(\max )=27.602$ & \\
\hline$R($ reflections $)=0.0$ & wR2 (reflections) & $=0.1169(4422)$ \\
\hline$S=1.055$ & Npar $=254$ & \\
\hline
\end{tabular}




\section{Copy of 1D and 2D NMR Spectra}

\section{${ }^{1} \mathrm{H},{ }^{13} \mathrm{C}$, DEPT-135 NMR spectra of $1 \mathrm{f}$ :}

ID-0915.017.001.1r.esp_KM-45-G-1H.esp<smiles>CCCCOC[C@H]1OC=C(I)C(=O)[C@H]1O</smiles>



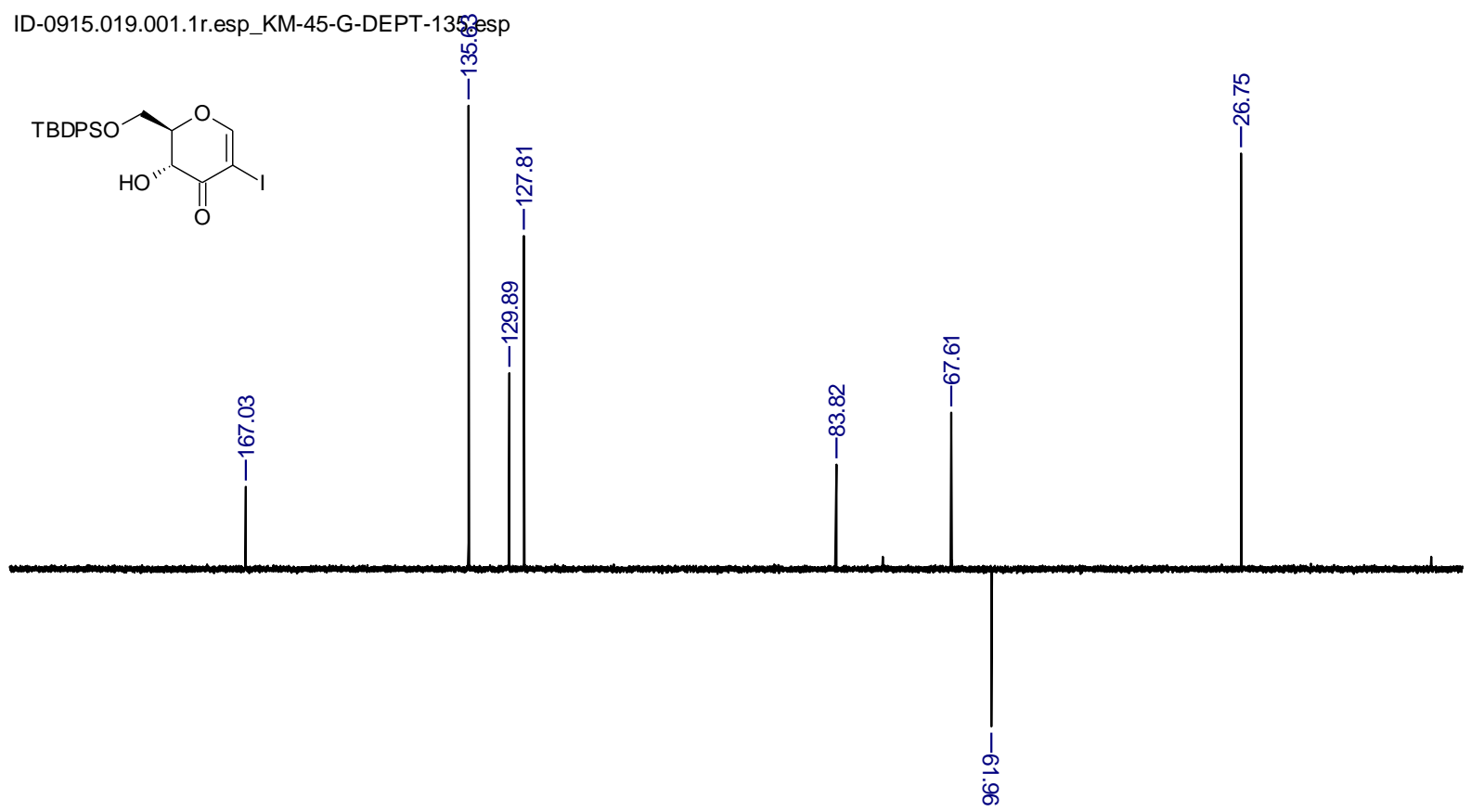



\section{${ }^{1} \mathrm{H},{ }^{13} \mathrm{C}$, DEPT-135 NMR spectra of $1 \mathrm{~g}$ :}

NMR data.041.001.1r.esp_FH-186-1H.esp<smiles>O=C1C(Br)=CO[C@H](COc2ccccc2)[C@@H]1Br</smiles>








\section{${ }^{1} \mathrm{H},{ }^{13} \mathrm{C}$, DEPT-135, DEPT-90 NMR spectra of 2a:}

ID-0715.031.001.1R_FH-98-1H_600.ESP<smiles>O=C(COCc1ccccc1)O[C@@H](C(=O)c1c[nH]c(-c2ccccc2)n1)[C@H](O)Cc1ccccc1</smiles>



FH-GA-1H-C.001.001.1r.esp<smiles>O=C(COCc1ccccc1)O[C@@H](C(=O)c1c[nH]c(-c2ccccc2)n1)[C@H](Cc1ccccc1)OCc1ccccc1</smiles>

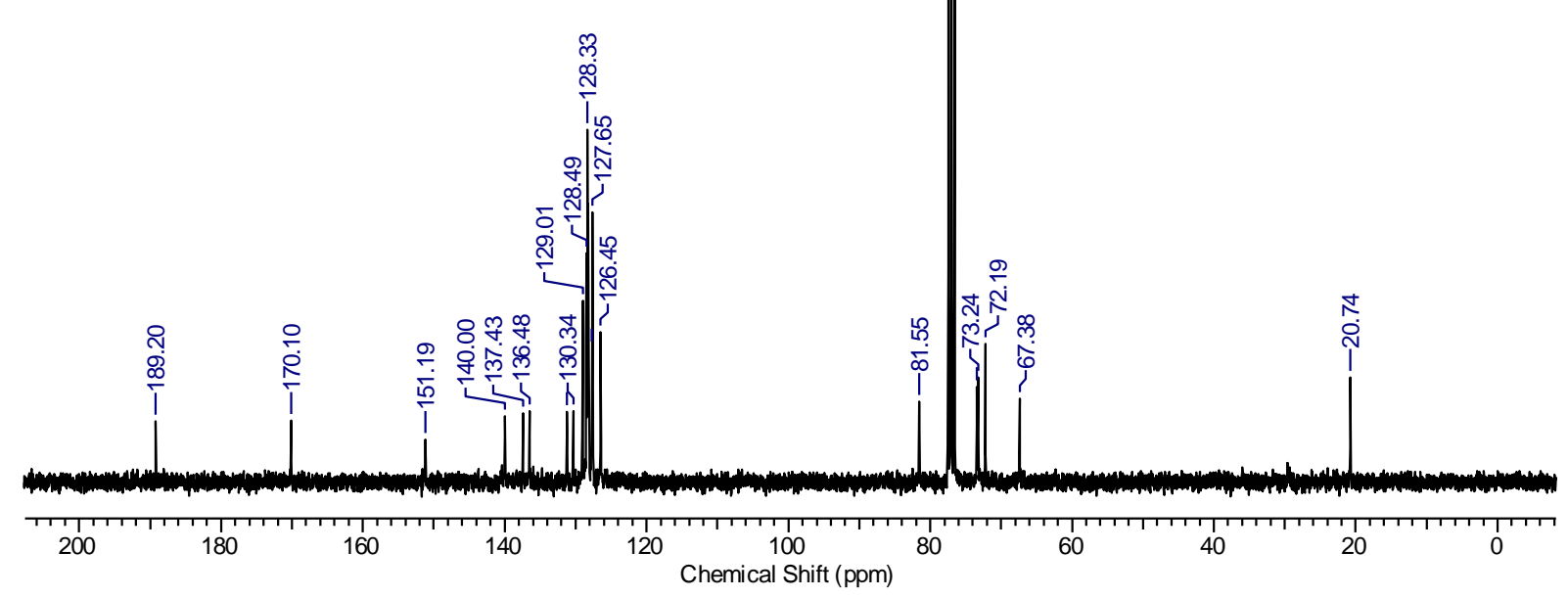




2D (COSY, HMBC, HSQC, and NOESY) NMR spectra of 2a:


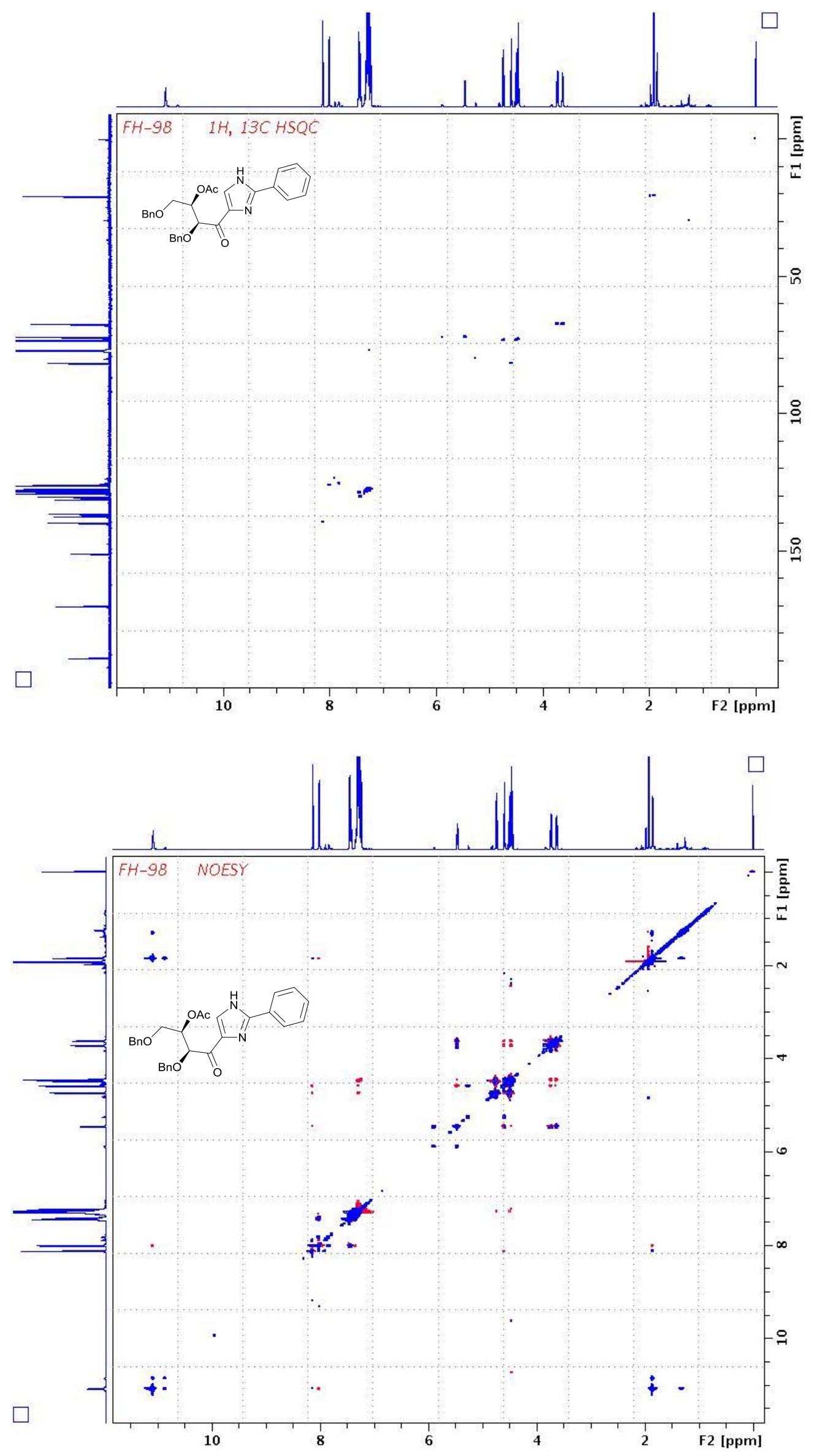


\section{${ }^{1} \mathrm{H},{ }^{13} \mathrm{C}$, DEPT-135, DEPT-90 NMR spectra of $2 \mathrm{~b}$ :}

FH-GL-1A-1H.001.001.1r.esp



FH-GL-1B-C.001.001.1r.esp<smiles>CO[C@H](COCc1ccccc1)[C@@H](OCc1ccccc1)C(=O)c1c[nH]c(-c2ccccc2)n1</smiles>

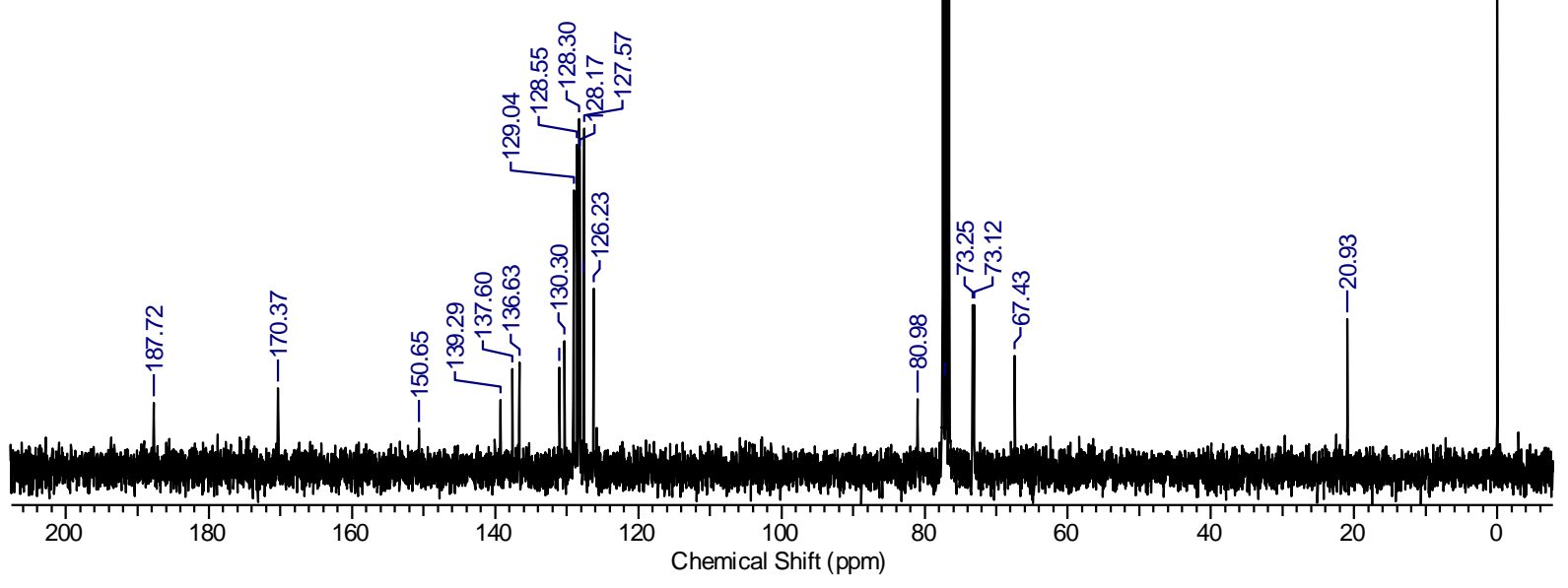


FH-GL-1B-C.002.001.1r.esp



FH-GL-1B-C.003.001.1r.esp



\section{${ }^{1} \mathrm{H},{ }^{13} \mathrm{C}$, DEPT-135, DEPT-90 NMR spectra of 2c:}

ID-0715.025.001.1R FH-152-XY-1H.ESP<smiles>O=C1c2nc(-c3ccccc3)[nH]c2OCC1[18O]</smiles>

<smiles>O=C(c1c[nH]c(-c2ccccc2)n1)C1COCC(O)C1=O</smiles>




ID-0715.027.001.1R_FH-152-XY-DEPT-135.ESP



ID-0715.028.001.1R_FH-152-XY-DEPT-90.ESP


S14 


\section{${ }^{1} \mathrm{H},{ }^{13} \mathrm{C}$, DEPT-135, DEPT-90 NMR spectra of 2d:}

FH-RH-1A-1H.001.001.1r.esp


FH-RH-1B-C.001.001.1r.esp





FH-RH-1B-C.003.001.1r.esp






\section{${ }^{1} \mathrm{H},{ }^{13} \mathrm{C}$, DEPT-135, DEPT-90 NMR spectra of 2e:}

FH-FU-1A-1H.001.001.1r.esp


FH-FU-1B-C.001.001.1r.esp




FH-FU-1B-C.003.001.1r.esp




${ }^{1} \mathrm{H},{ }^{13} \mathrm{C}$, DEPT-135, DEPT-90 NMR spectra of $2 \mathrm{f}$ :

FH-162B-1H.001.001.1R.ESP<smiles>COc1ccc(-c2nc(C(=O)C(OCc3ccccc3)C(COc3ccccc3)OC(=O)c3ccccc3)c[nH]2)cc1</smiles>



FH-162-C.001.001.1r.esp<smiles>COc1ccc(-c2nc(C(=O)C(OCc3ccccc3)C(COCc3ccccc3)OC(=O)c3ccccc3)c[nH]2)cc1</smiles>








FH-162-C.003.001.1r.esp


S20 
${ }^{1} \mathrm{H},{ }^{13} \mathrm{C}$, DEPT-135, DEPT-90 NMR spectra of 2g:

.045.001.1R_FH-163-1H.ESP<smiles>COc1ccc(-c2nc(C(=O)C(OCc3ccccc3)C(COc3ccccc3)OC(C)=O)c[nH]2)cc1</smiles>



.046.001.1R_FH-163-13C.ESP<smiles>COc1ccc(-c2nc(C(=O)C(OCc3ccccc3)C(Cc3ccccc3)OCc3ccccc3)c[nH]2)cc1</smiles>








.048.001.1R FH-163-DEPT-90.ESP<smiles>COc1ccc(-c2nc(C(=O)[C@@H](OCc3ccccc3)[C@@H](COCc3ccccc3)OC(C)=O)c[nH]2)cc1</smiles>

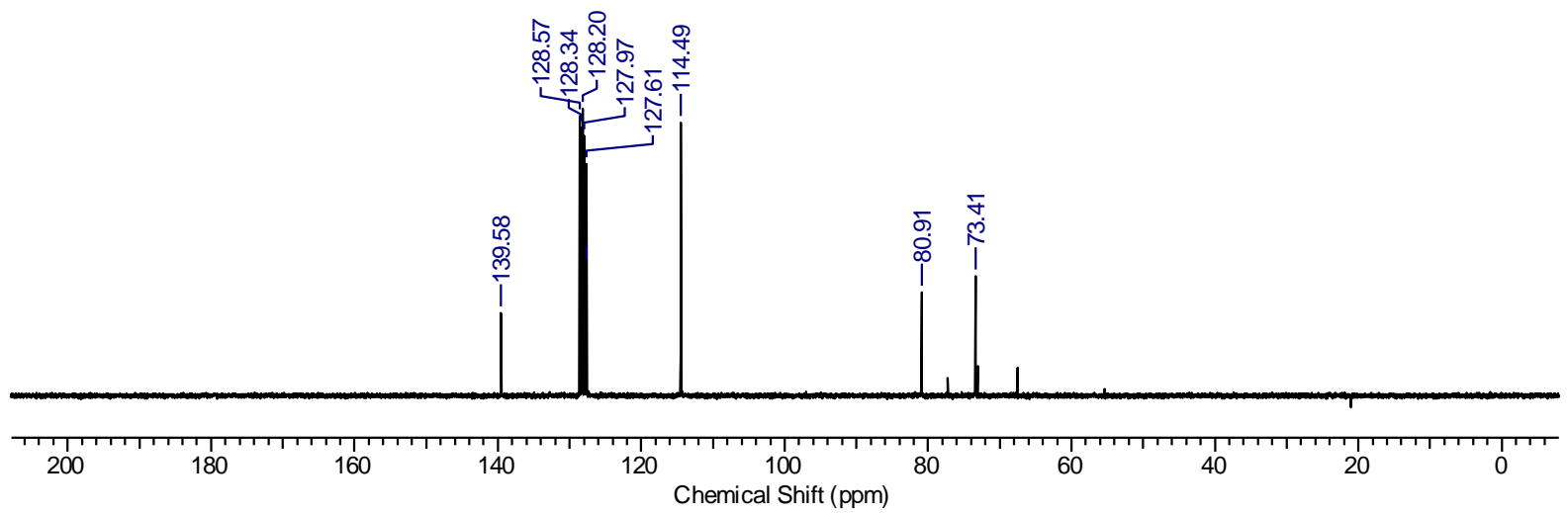


2D (COSY, HMBC, HSQC, and NOESY) NMR spectra of 2g:



${ }^{1} \mathrm{H},{ }^{13} \mathrm{C}$, DEPT-135, DEPT-90 NMR spectra of $2 \mathrm{~h}$ :

FH-164-1H.001.001.1r.esp


FH-164B-C.001.001.1r.esp<smiles>COc1ccc(-c2nc(C(=O)[C@@H](C)O)c[nH]2)cc1</smiles>






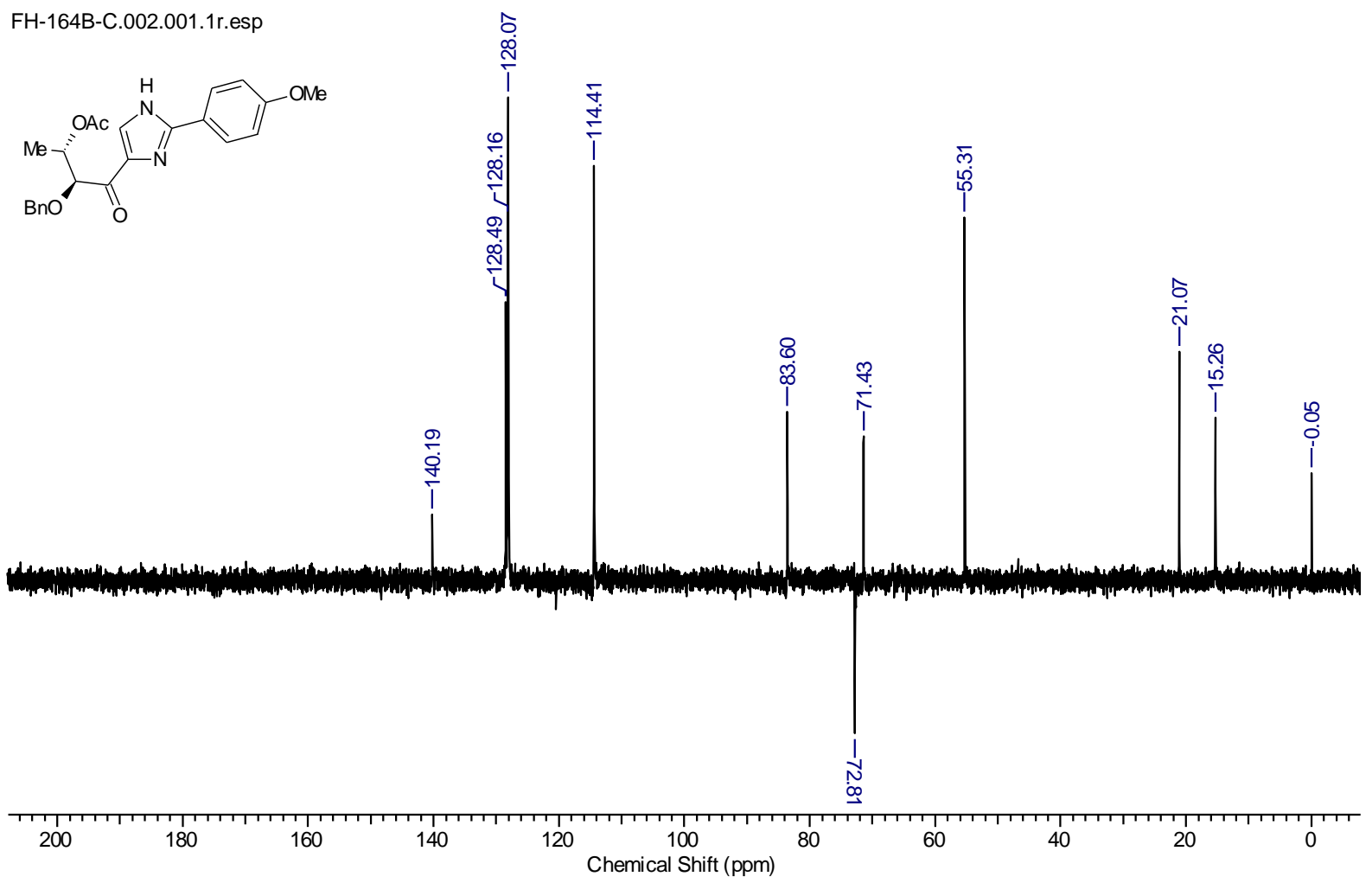

FH-164B-C.003.001.1r.esp

${ }^{1} \mathrm{H},{ }^{13} \mathrm{C}$, DEPT-135 NMR spectra of $2 \mathrm{i}$ :

.061.001.1r_KM-95-1H.esp
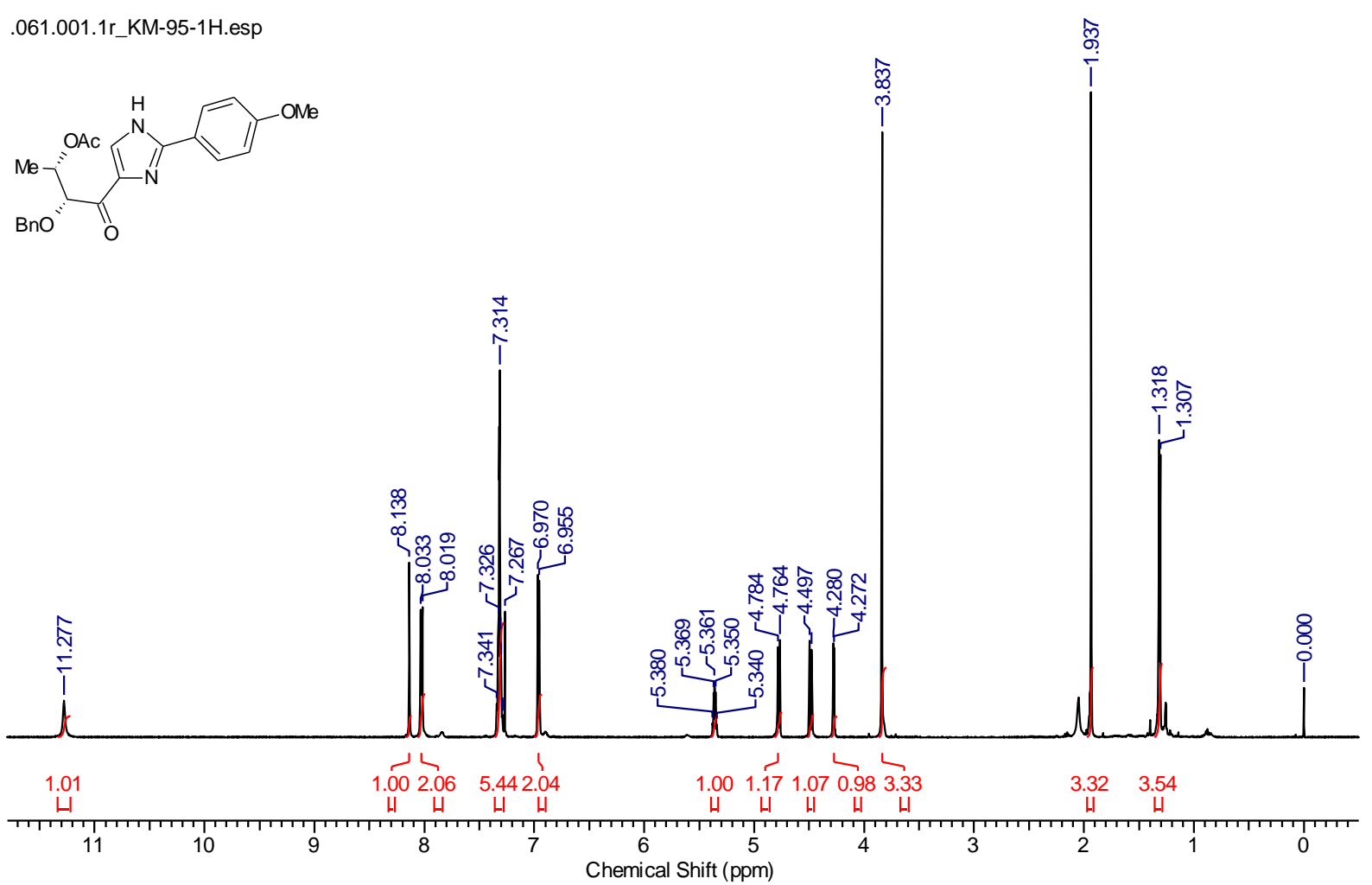

.062.001.1r_KM-95-13C.esp
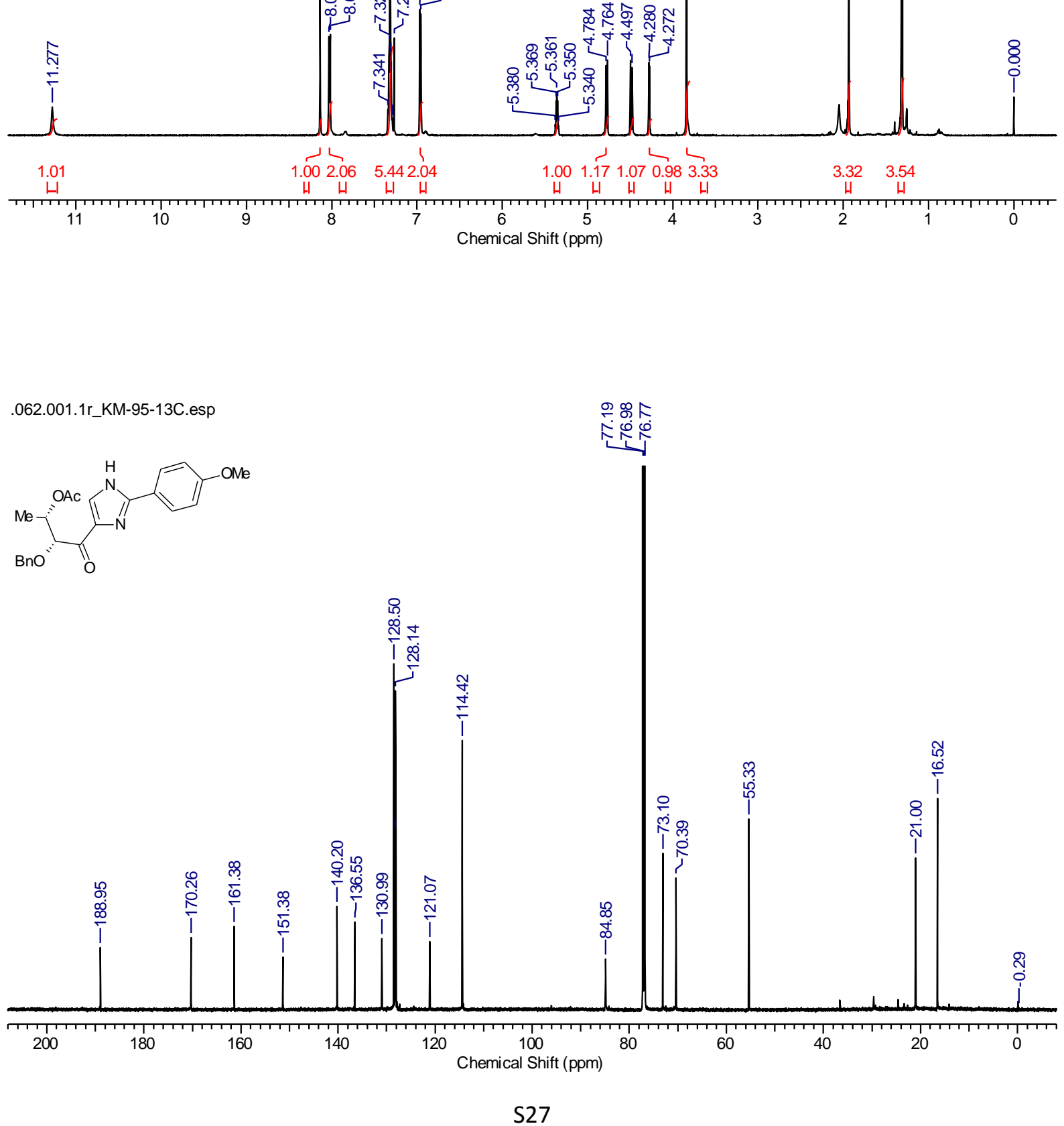
.063.001.1r_KM-95-DEPT-135.esp

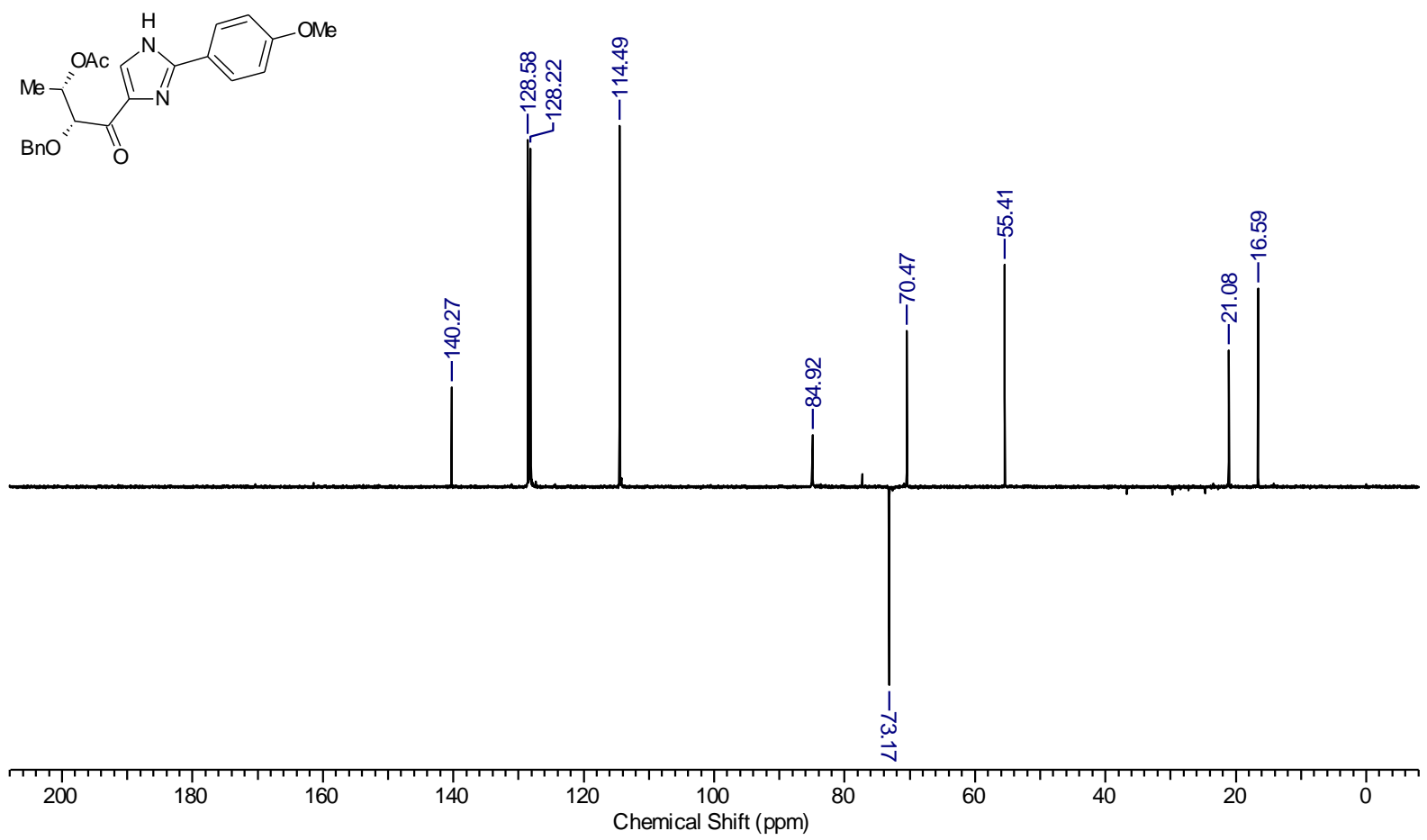

2D (COSY, HMBC, HSQC, and NOESY) NMR spectra of 2i:

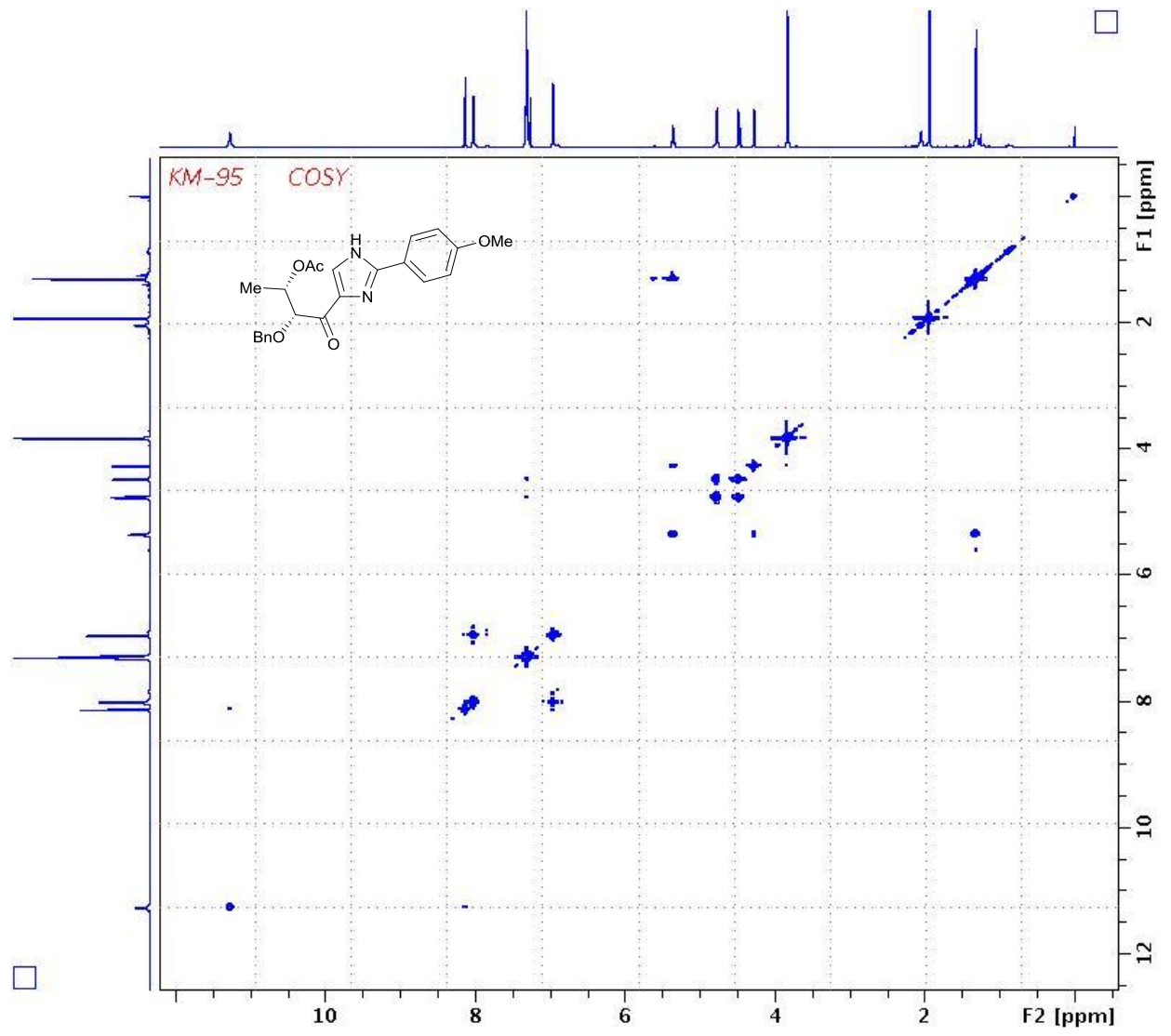



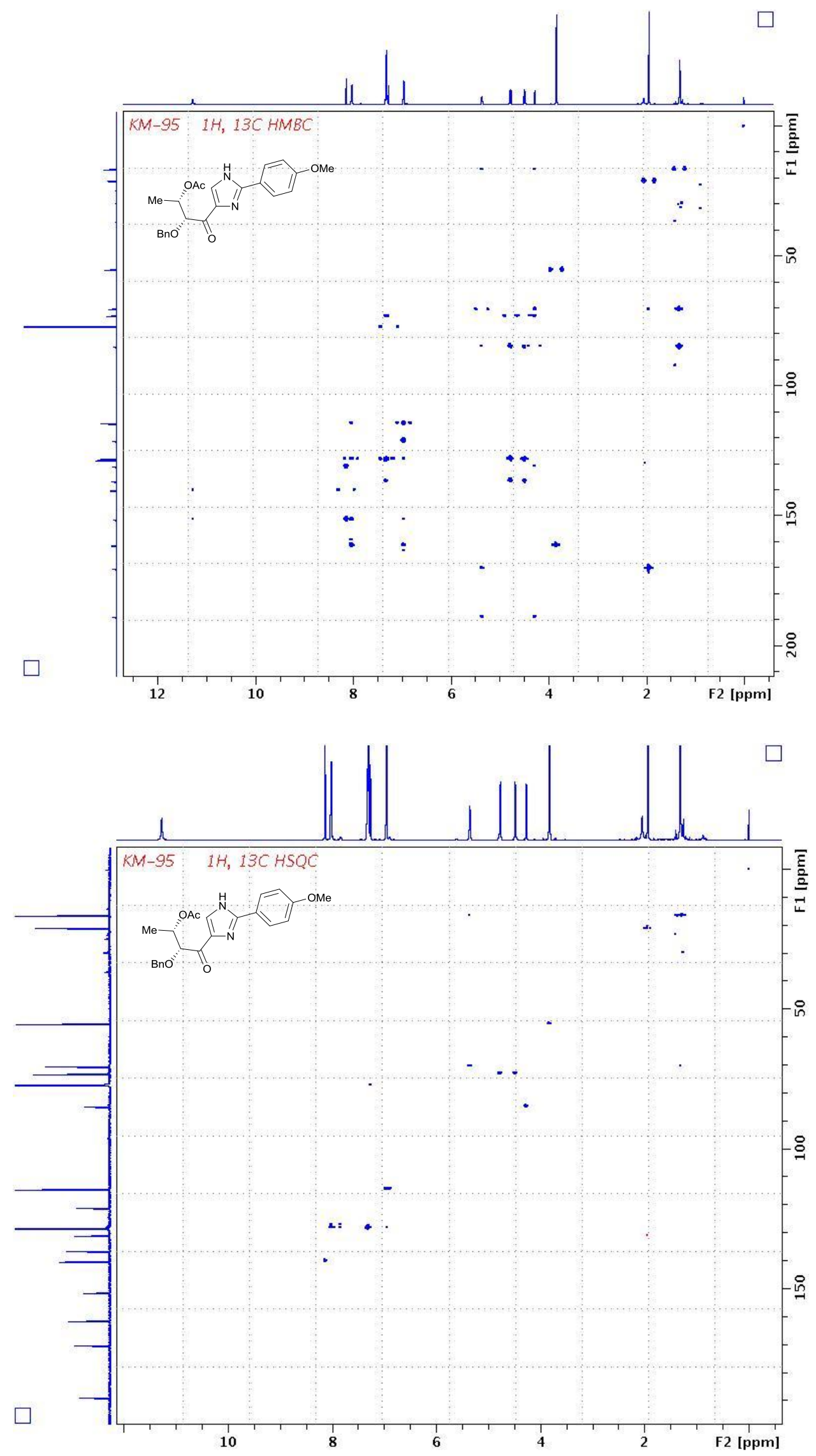


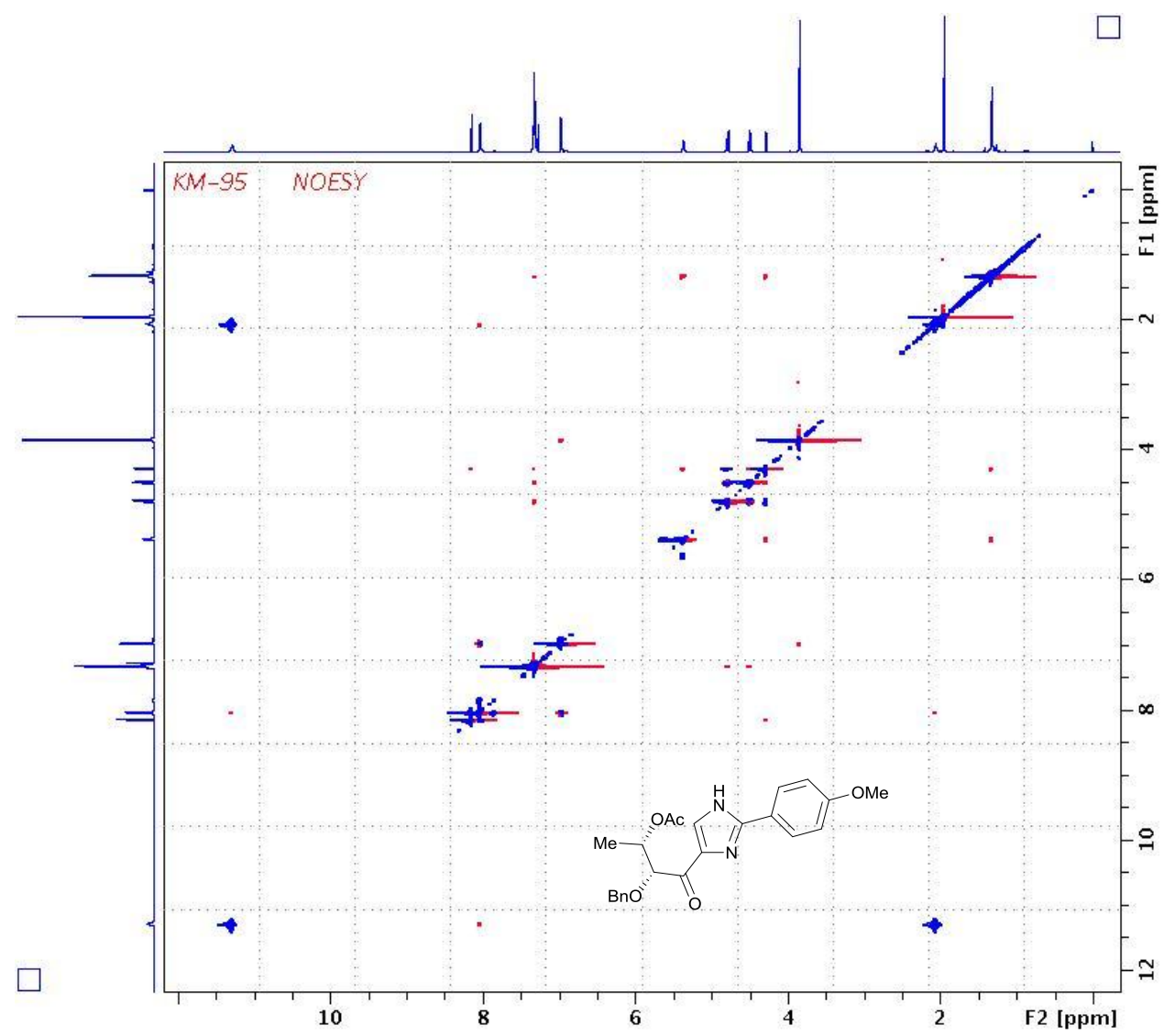

${ }^{1} \mathrm{H},{ }^{13} \mathrm{C}$, DEPT-135, DEPT-90 NMR spectra of $2 \mathrm{j}$ :

FH-GA-1A-1H.002.001.1r.esp

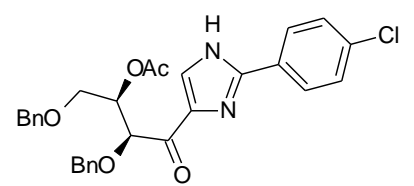

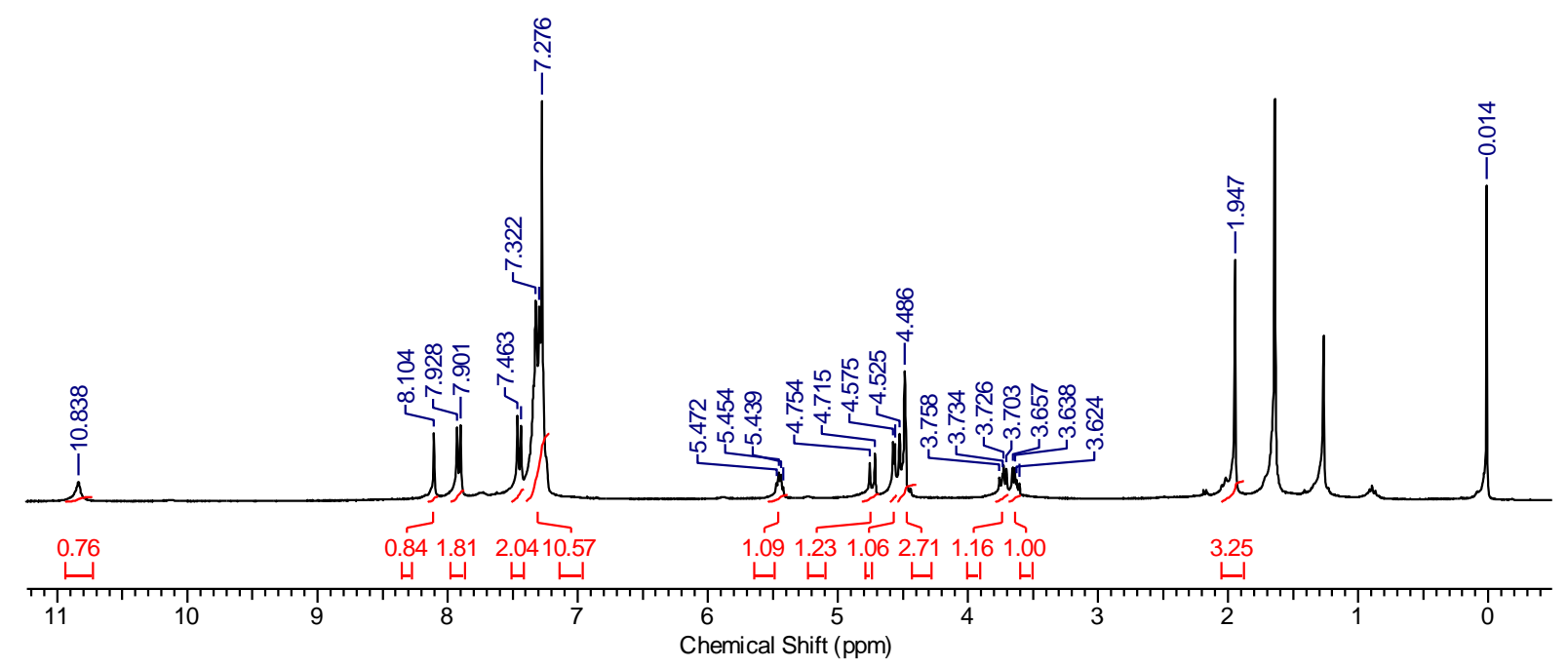




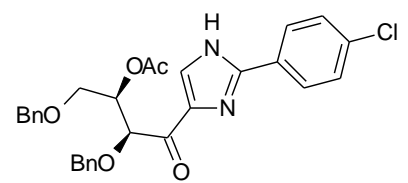
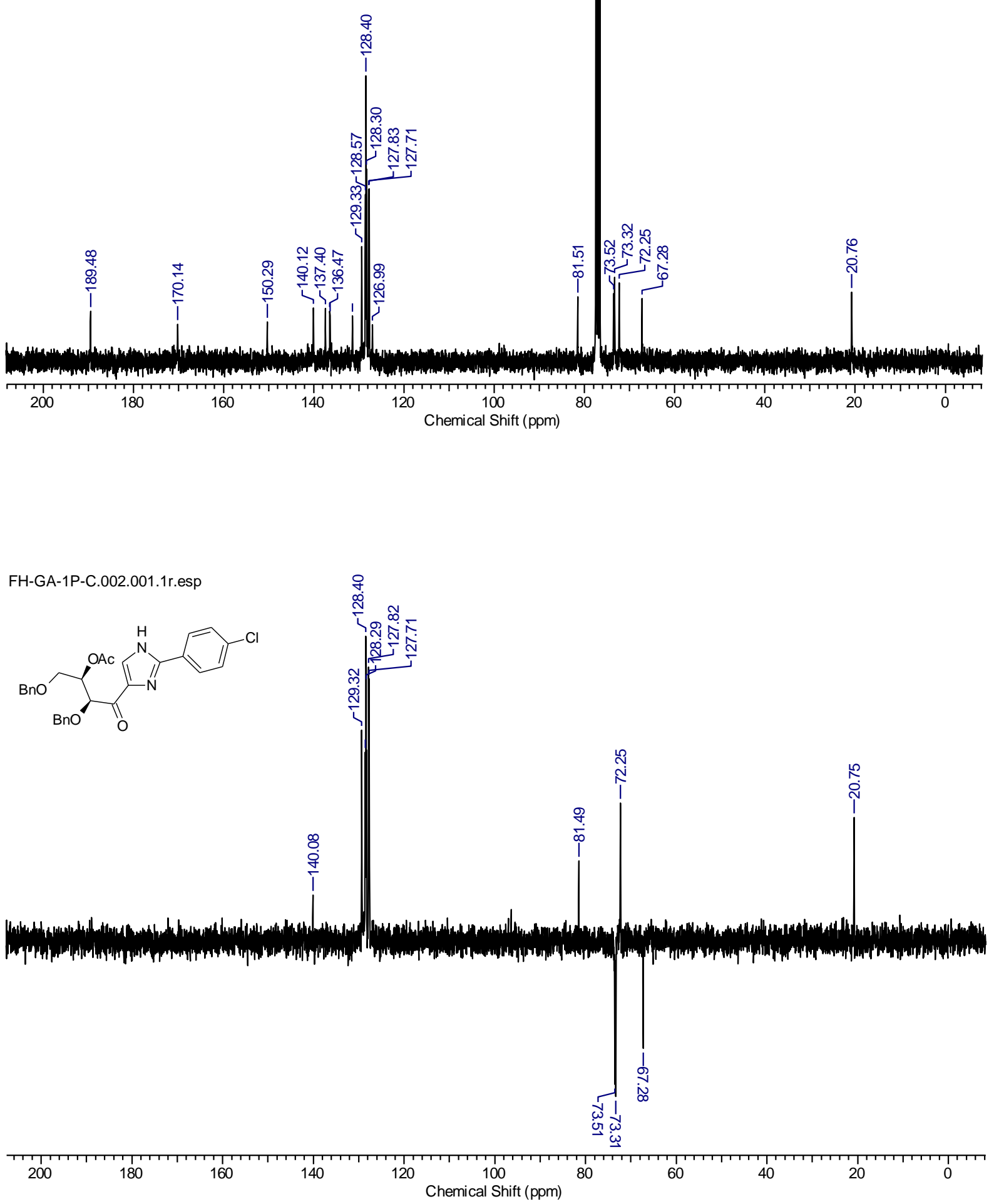
FH-GA-1P-C.003.001.1r.esp
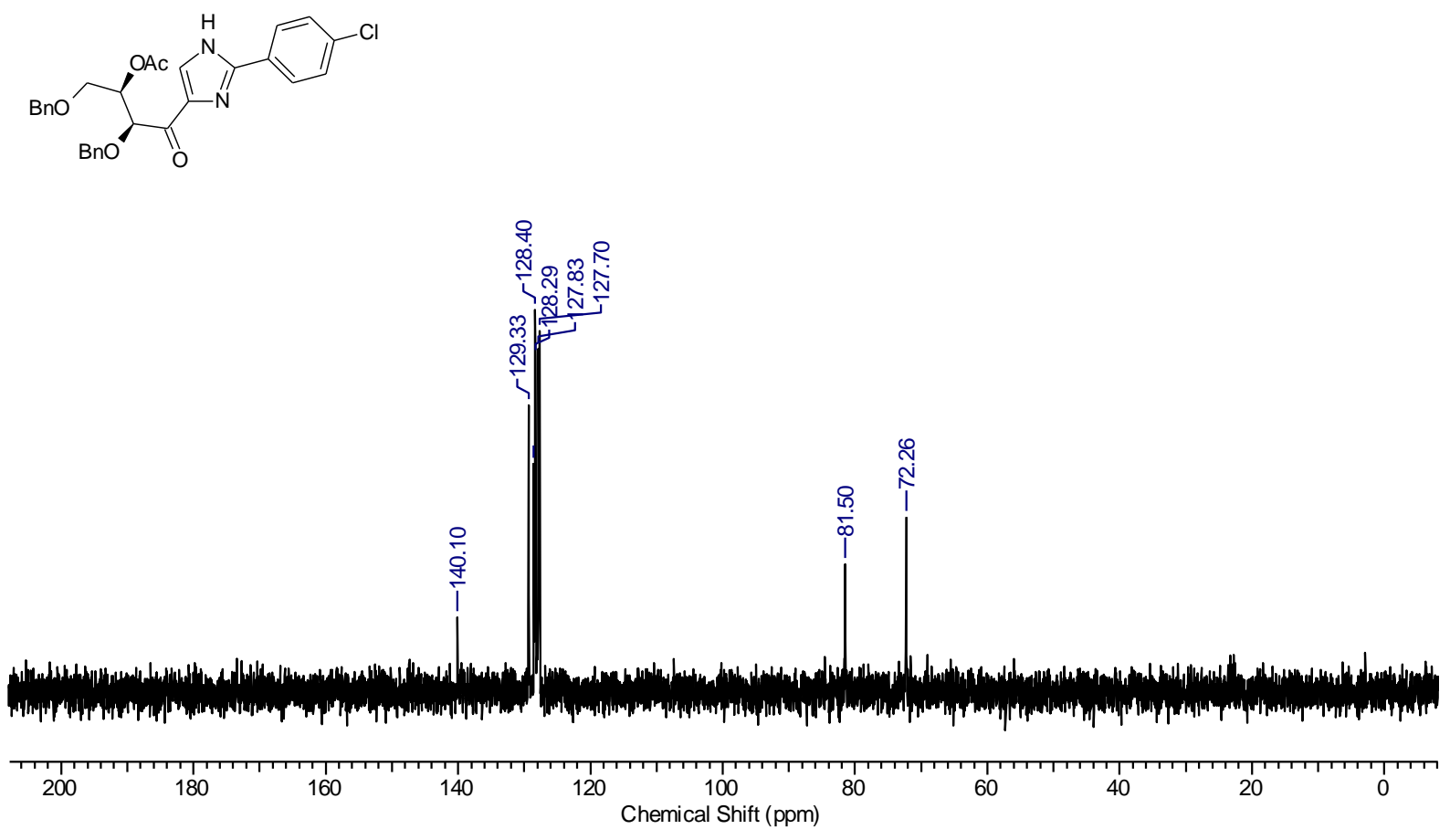

${ }^{1} \mathrm{H},{ }^{13} \mathrm{C}$, DEPT-135, DEPT-90 NMR spectra of $2 \mathrm{k}$ :

FH-GL-2A-1H.001.001.1r.esp
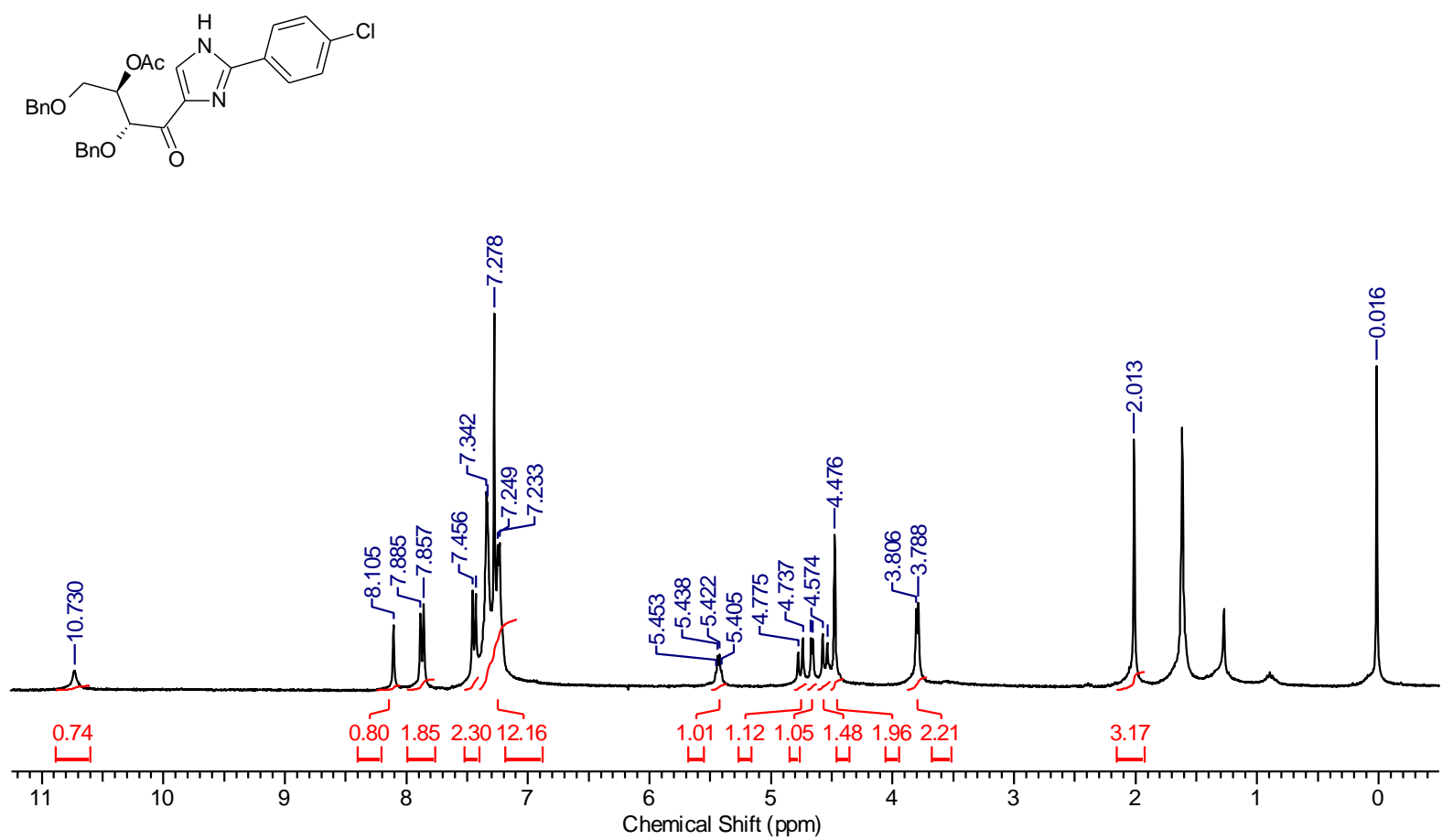

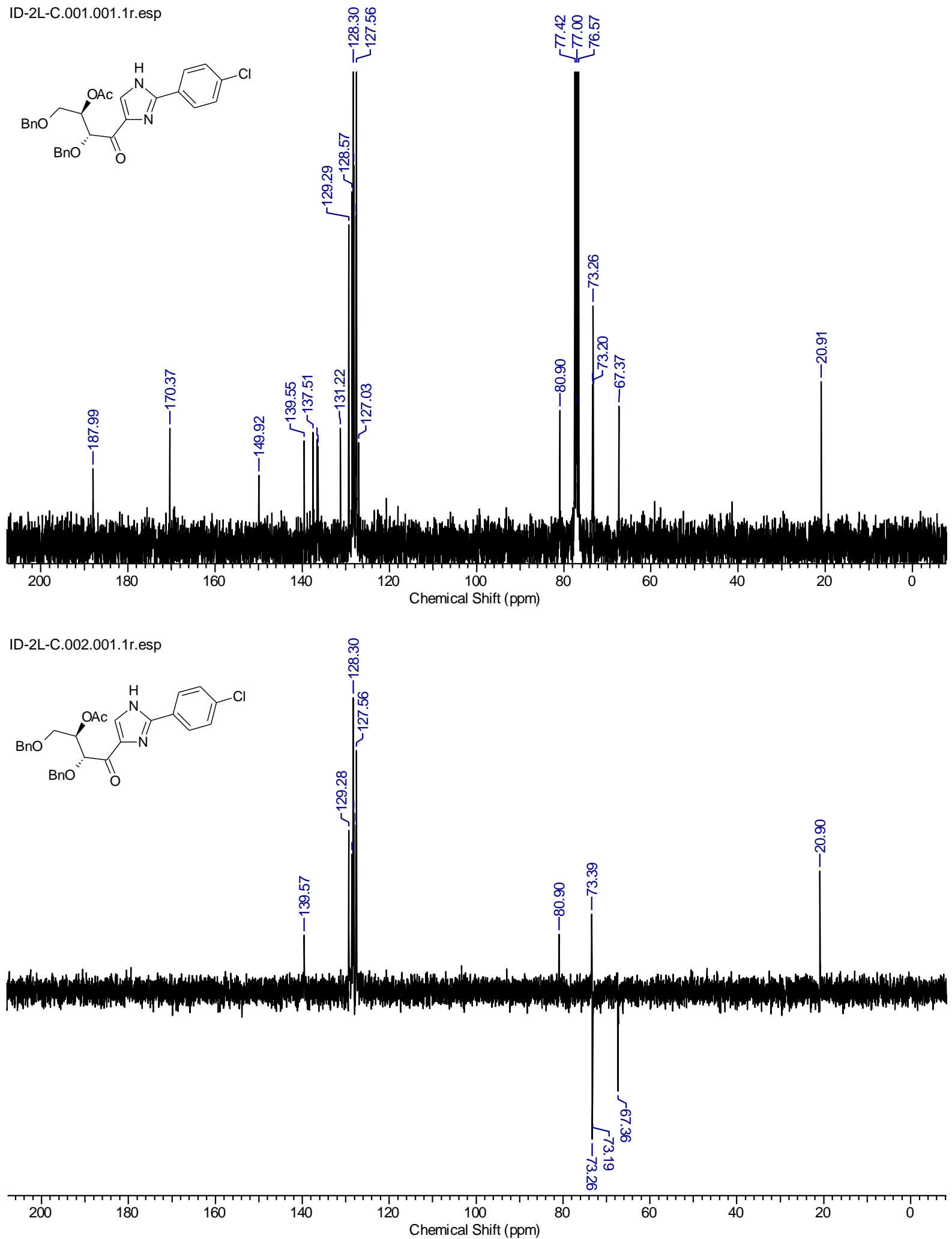


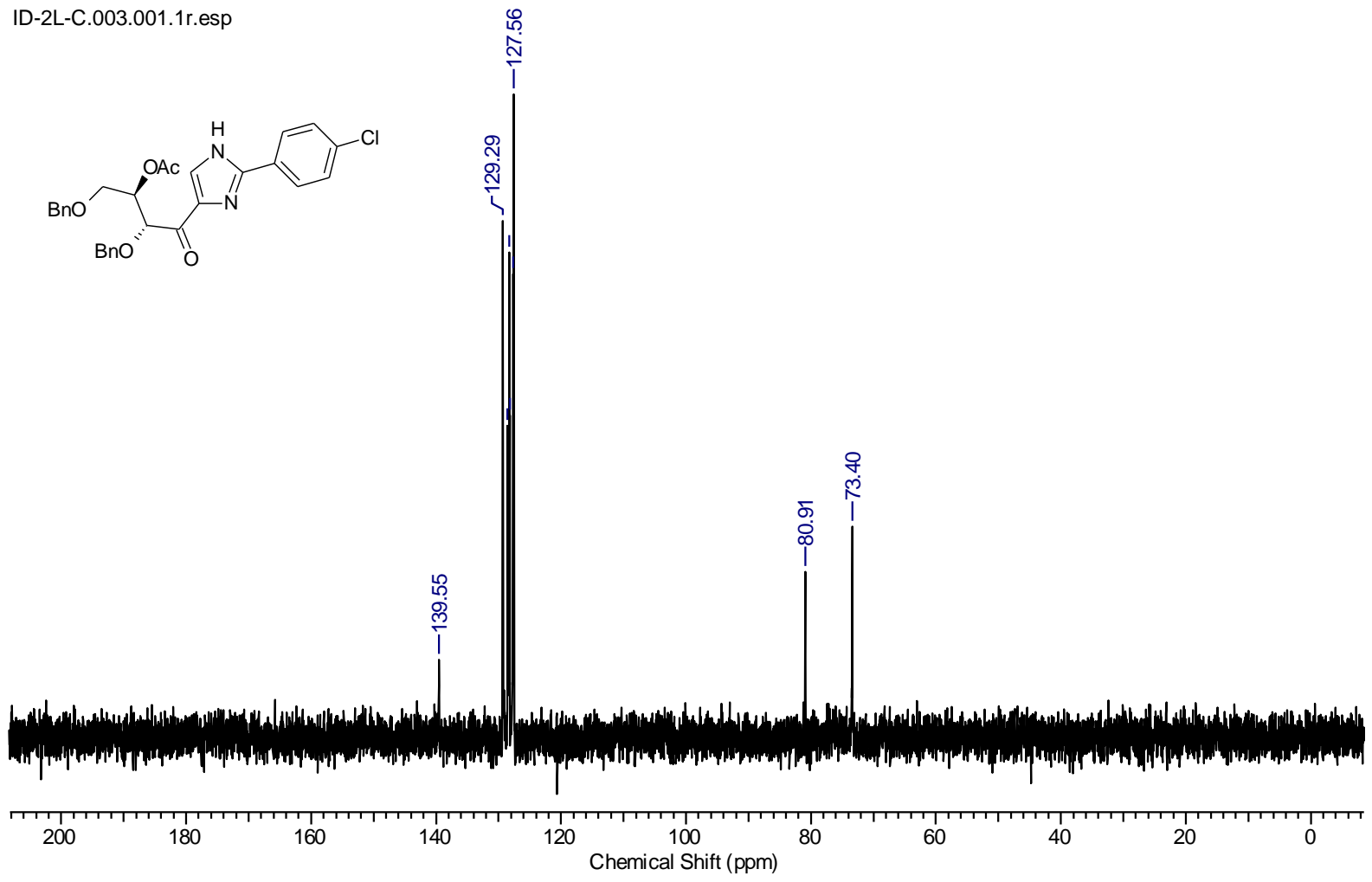

\section{${ }^{1} \mathrm{H},{ }^{13} \mathrm{C}$, DEPT-135, DEPT-90 NMR spectra of 2I:}

FH-RH-2A-1H.001.001.1r.esp

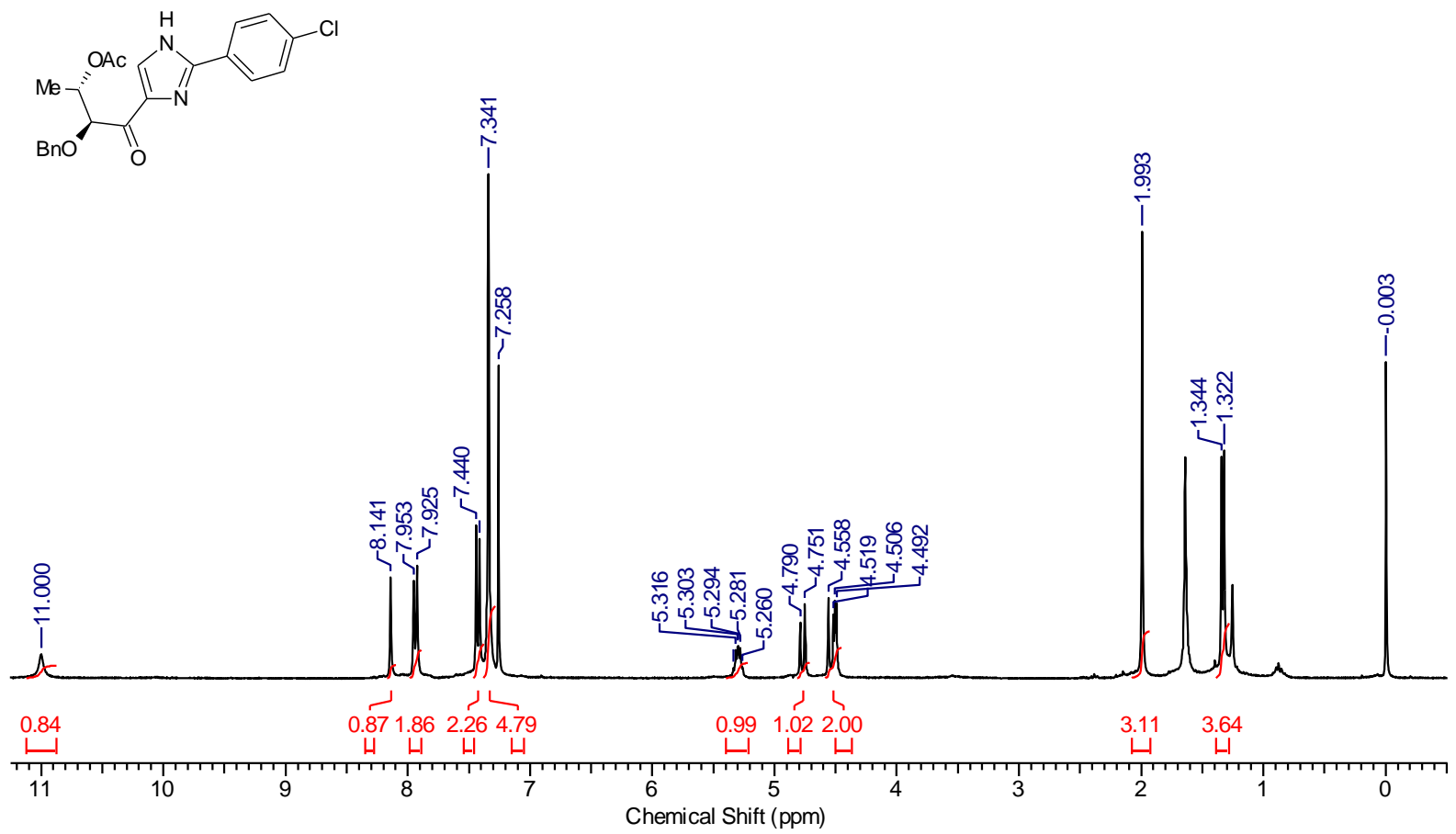



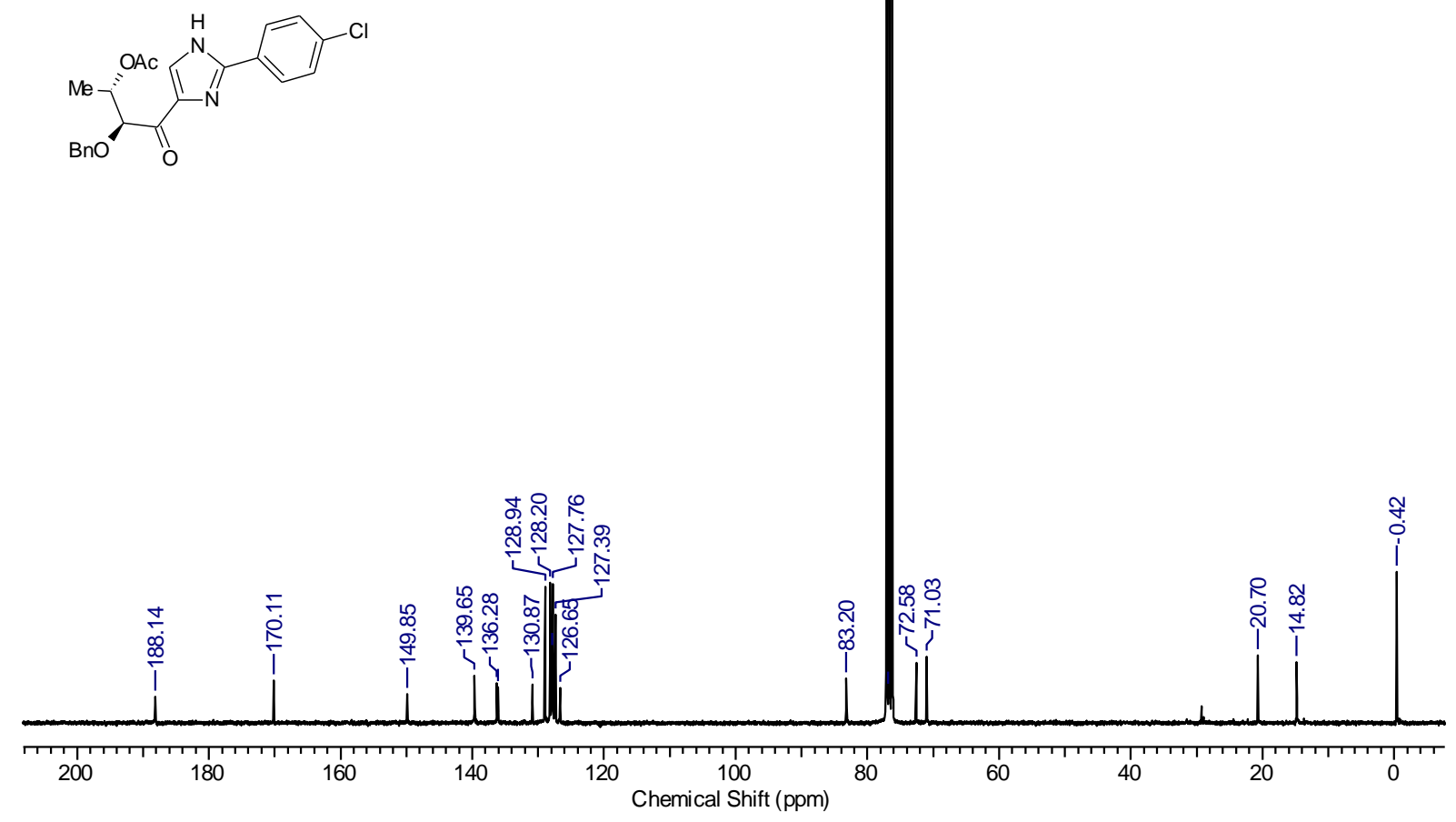

FH-RH-2B-C.002.001.1r.esp

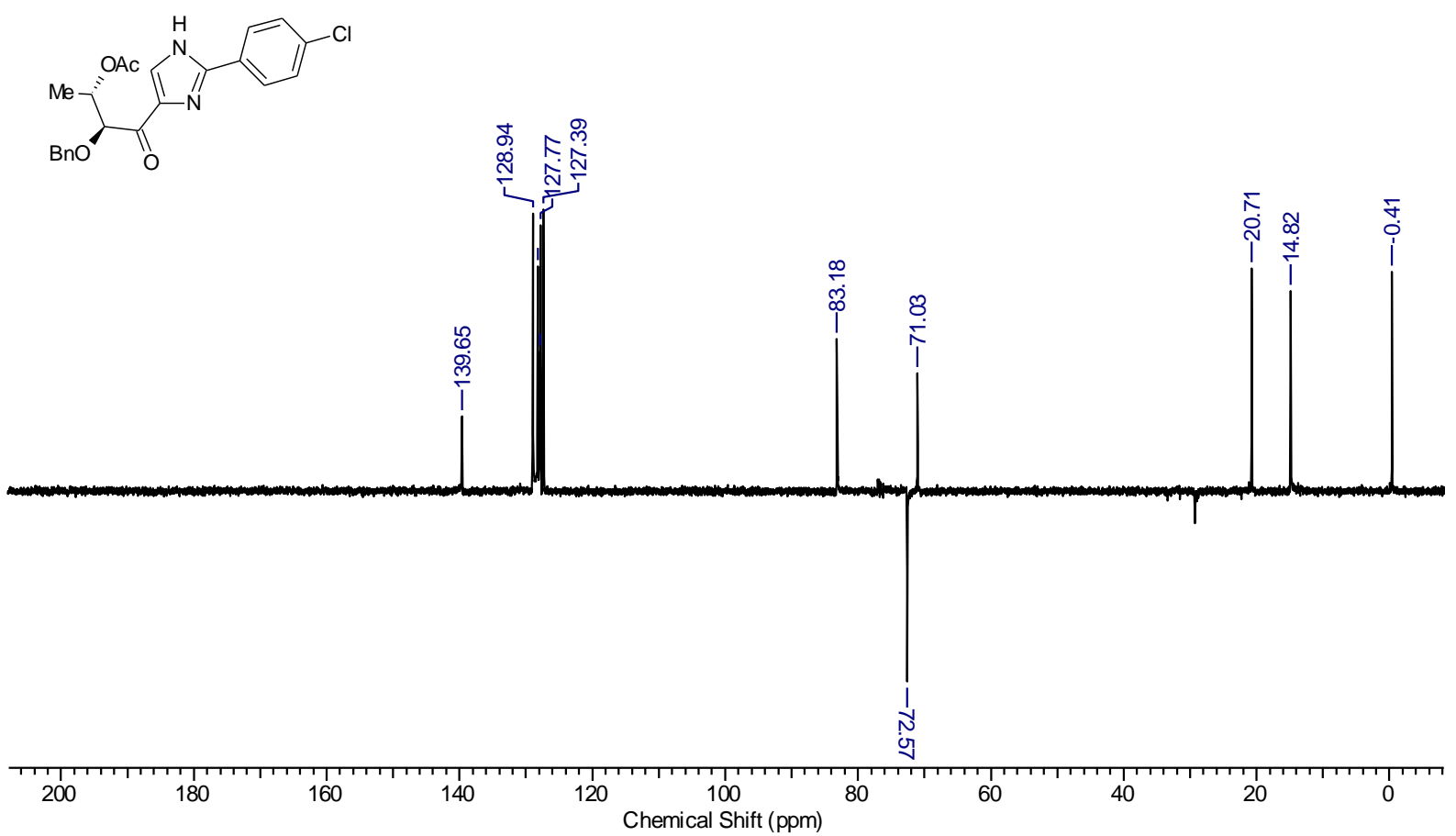


FH-RH-2B-C.003.001.1r.esp<smiles>C[C@H](OCc1ccccc1)[C@@H](O)C(=O)c1c[nH]c(-c2ccc(Cl)cc2)n1</smiles>

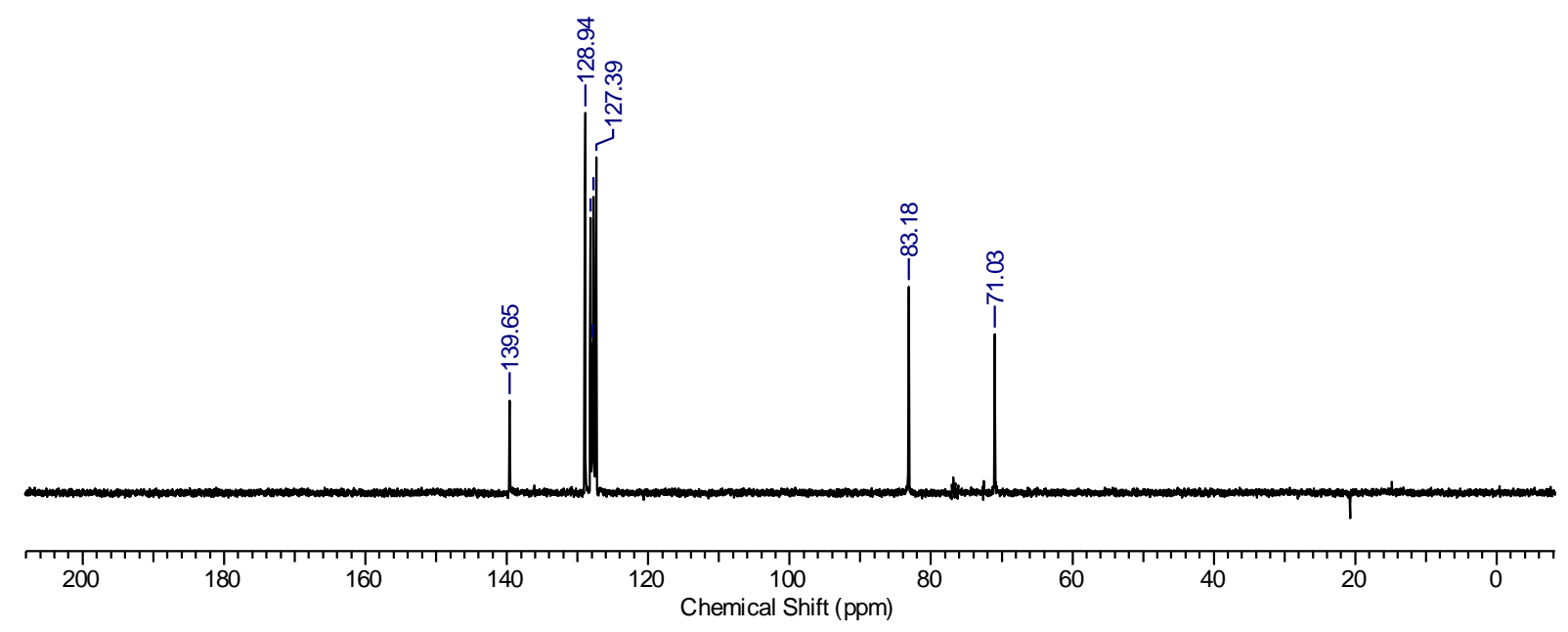

${ }^{1} \mathrm{H},{ }^{13} \mathrm{C}$, DEPT-135, DEPT-90 NMR spectra of $2 \mathrm{~m}$ :

FH-FU-2A-1H.001.001.1r.esp
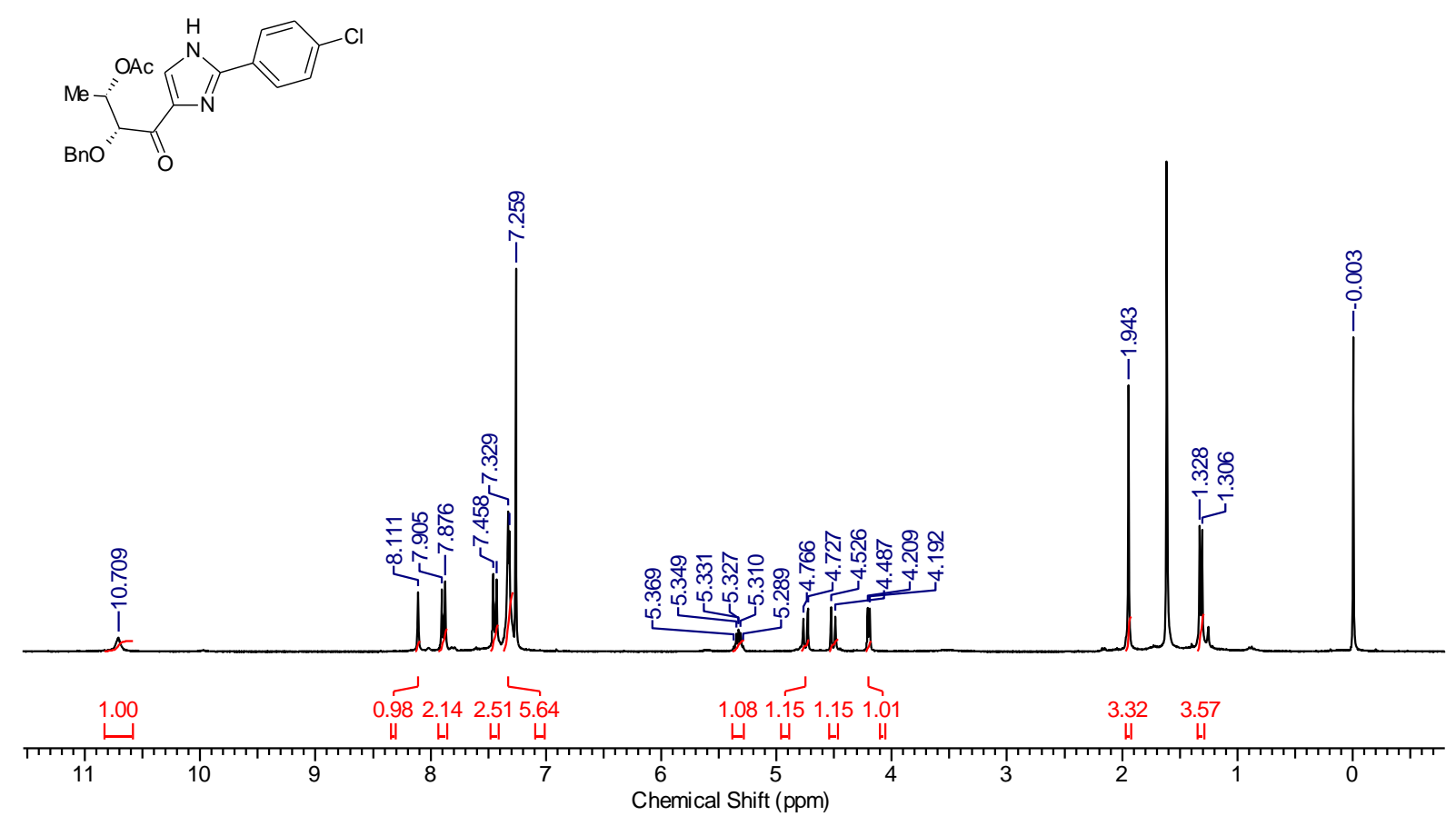

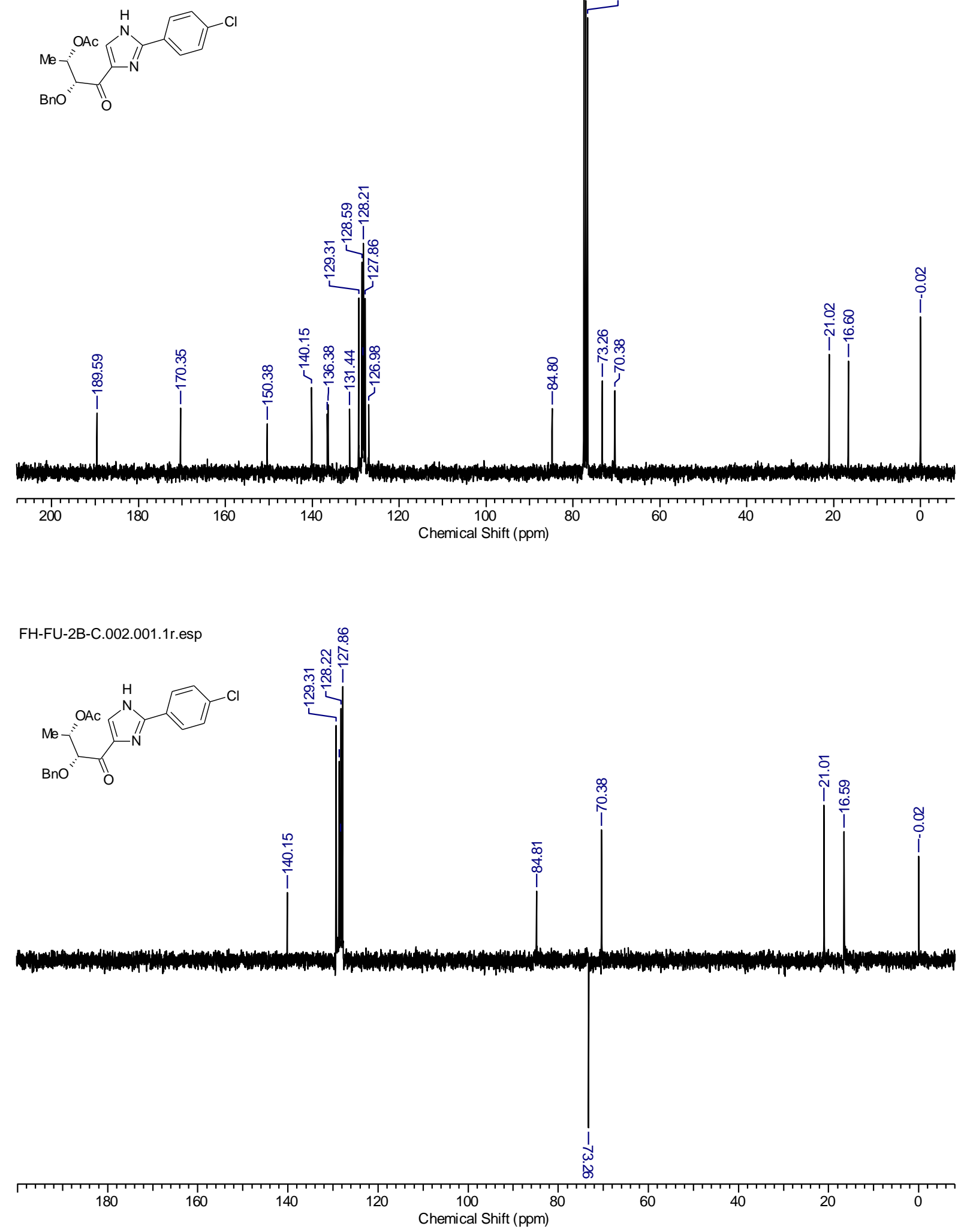

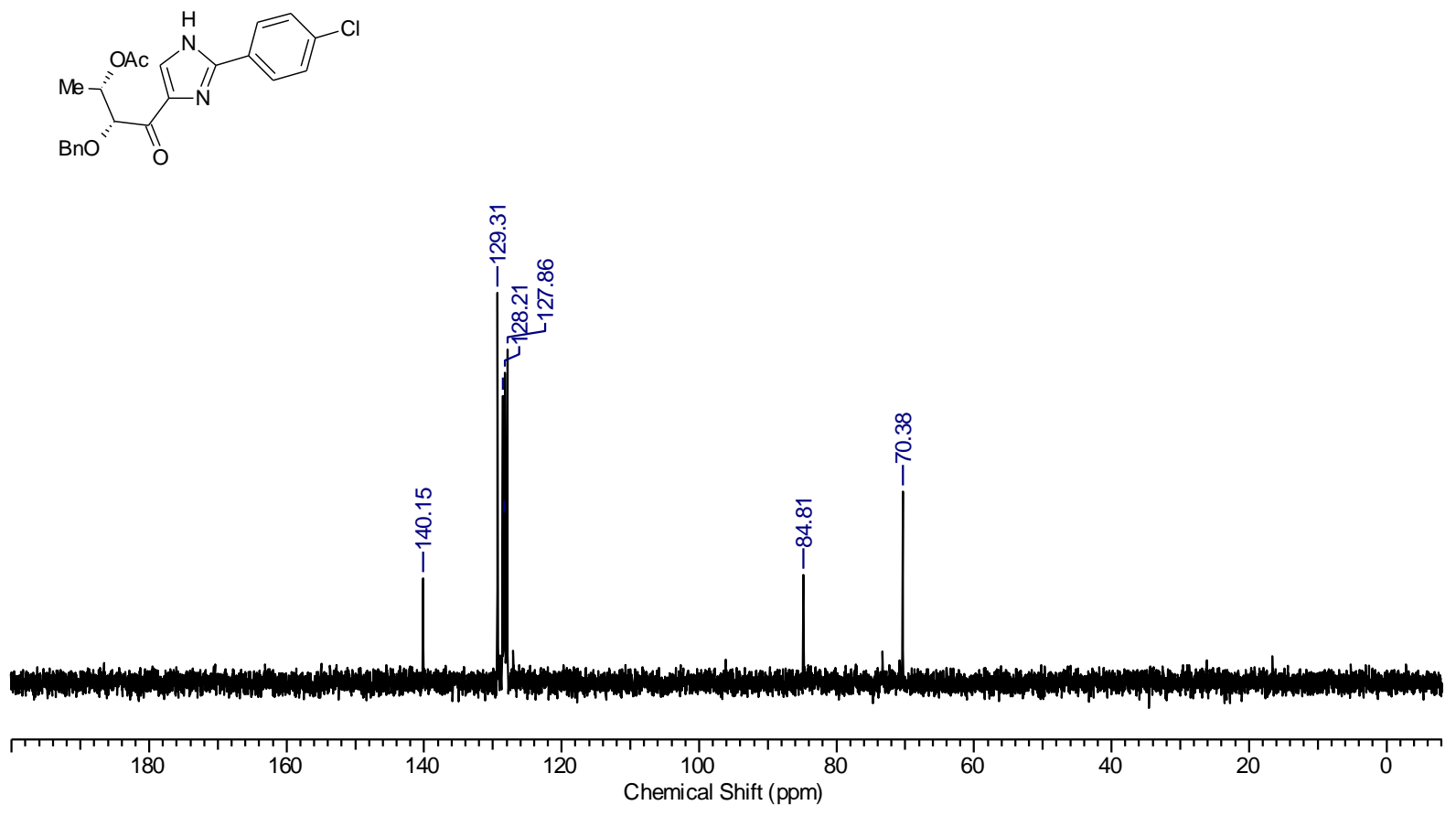

\section{${ }^{1} \mathrm{H},{ }^{13} \mathrm{C}$, DEPT-135, DEPT-90 NMR spectra of 2n:}

FH-GA-5A-1H.001.001.1r.esp
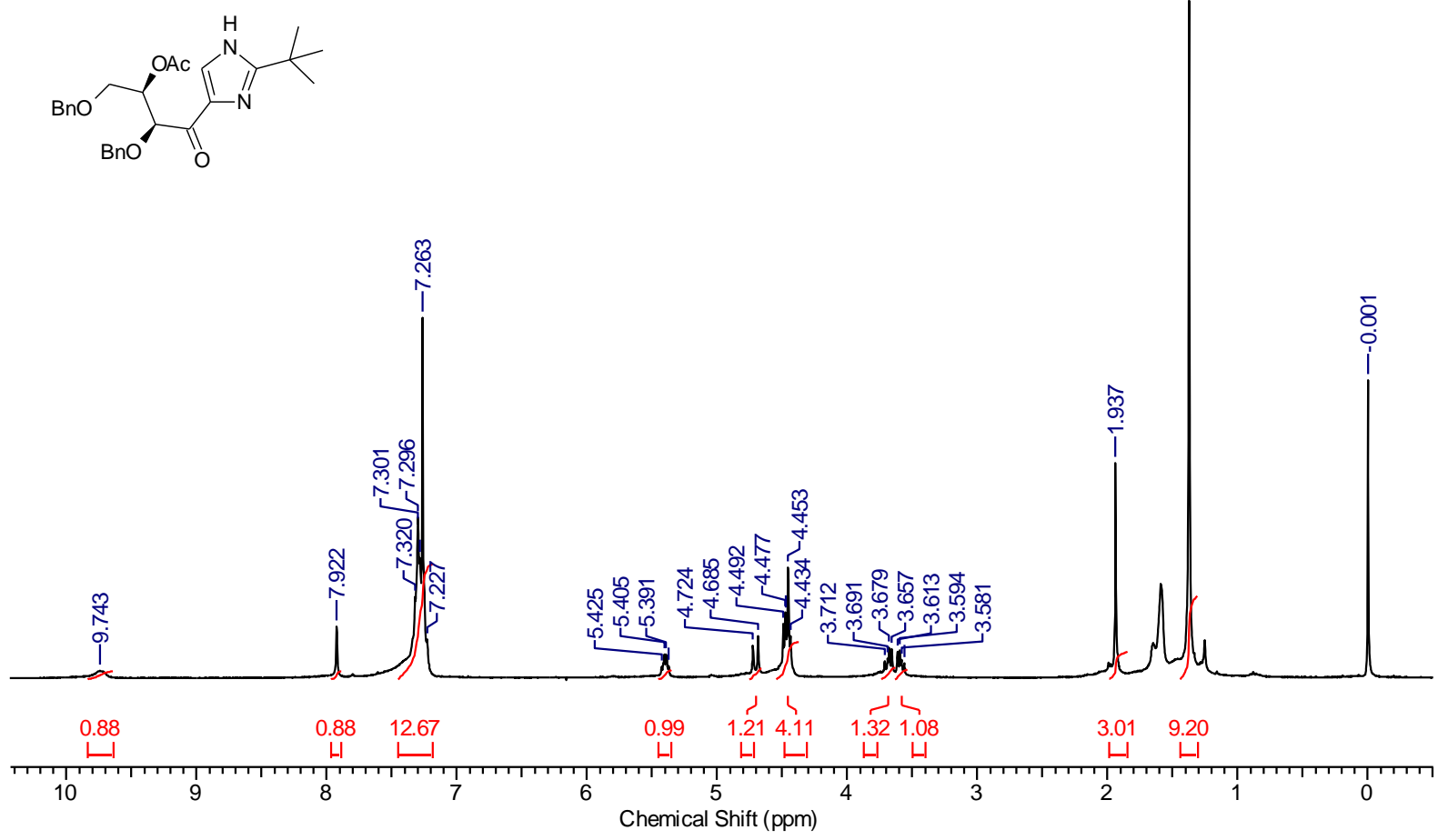
<smiles>CC(=O)O[C@H](COCc1ccccc1)[C@@H](OCc1ccccc1)C(=O)c1c[nH]c(C(C)(C)C)n1</smiles>

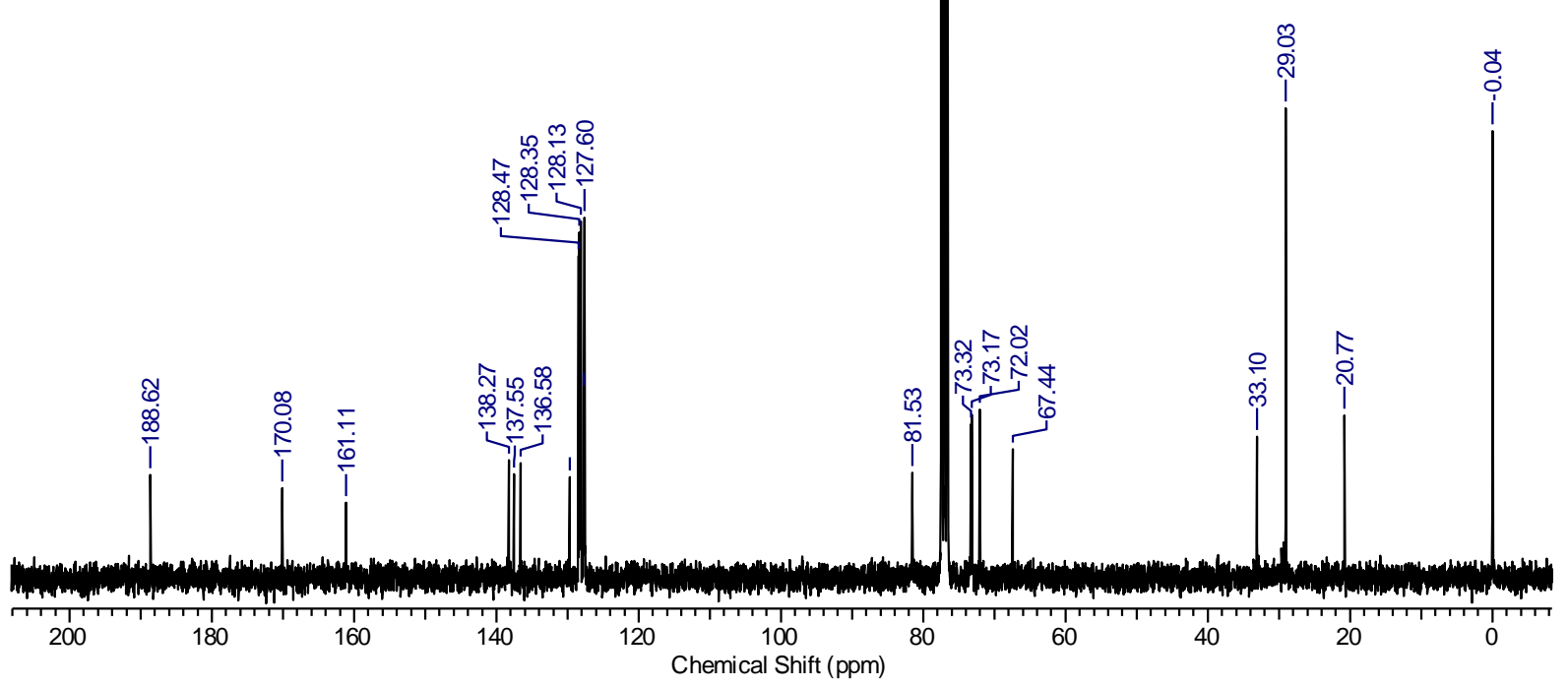

FH-GA-5B-C.002.001.1r.esp<smiles>CC(=O)O[C@@H](COCc1ccccc1)[C@@H](OCc1ccccc1)C(=O)c1c[nH]c(C(C)(C)C)n1</smiles>

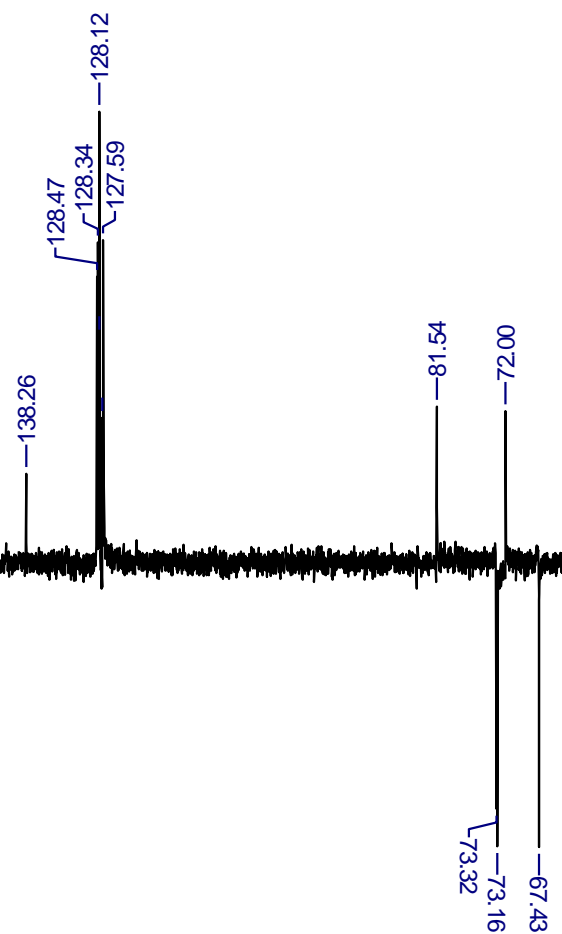






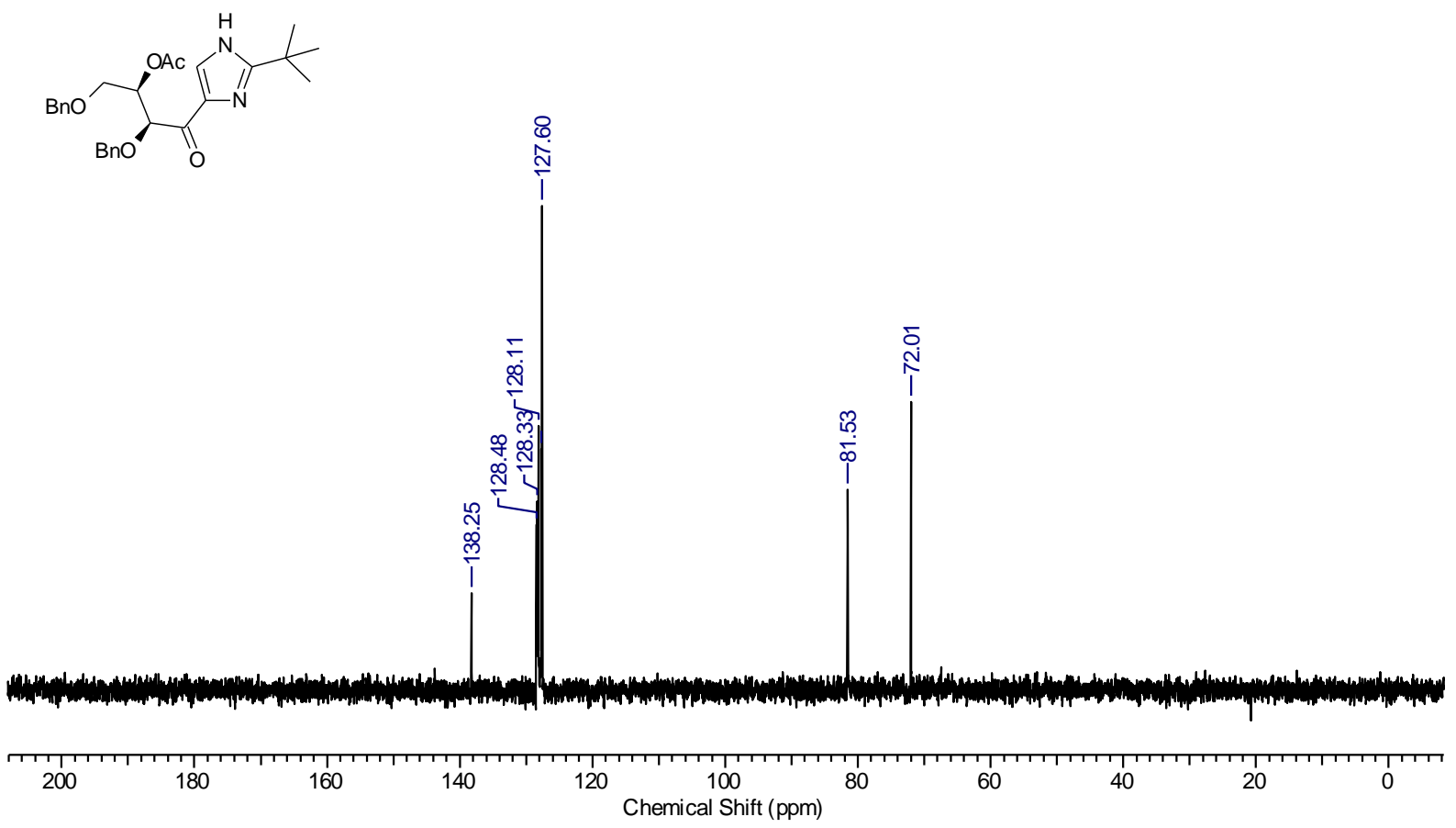

\section{${ }^{1} \mathrm{H},{ }^{13} \mathrm{C}$, DEPT-90 NMR spectra of 20:}

FH-GL-4A-1H.001.001.1r.esp
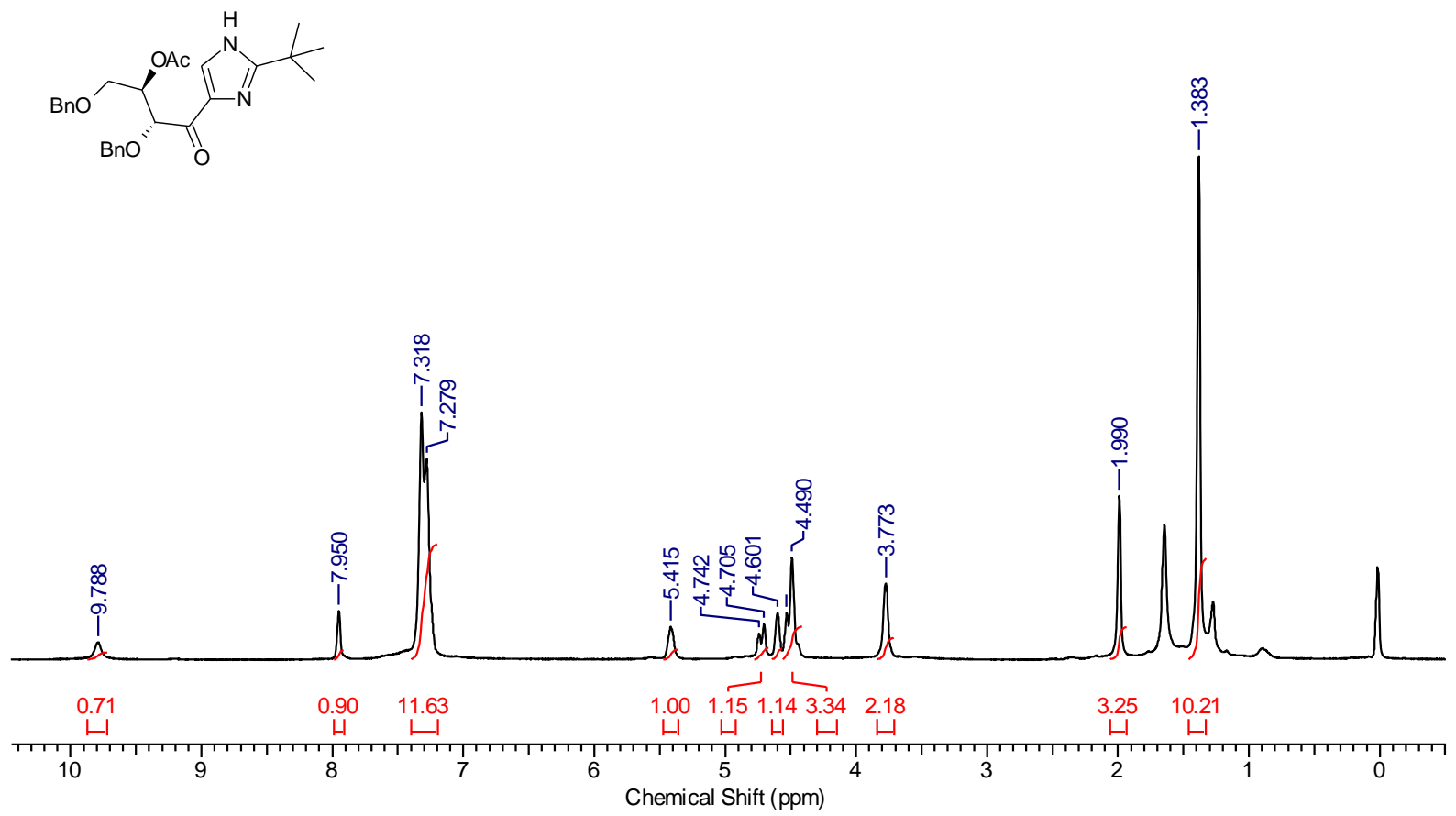
<smiles>CC(C)(C)c1nc(C(=O)[C@H](OCc2ccccc2)C(O)Cc2ccccc2)c[nH]1</smiles>
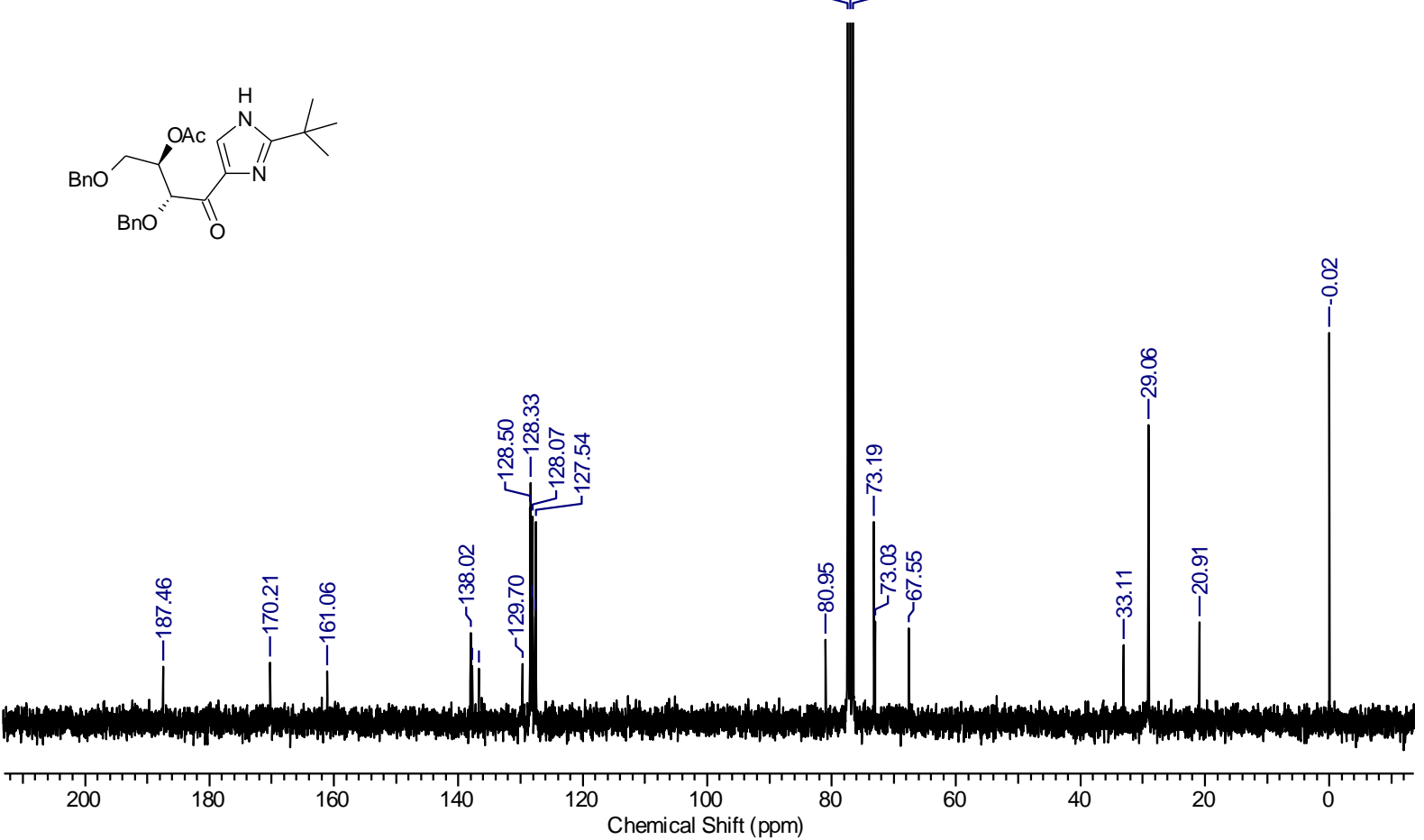

FH-GL-4B-C.003.001.1r.esp<smiles>CC(=O)O[C@@H](COc1ccccc1)[C@@H](OCc1ccccc1)C(=O)c1c[nH]c(C(C)(C)C)n1</smiles>

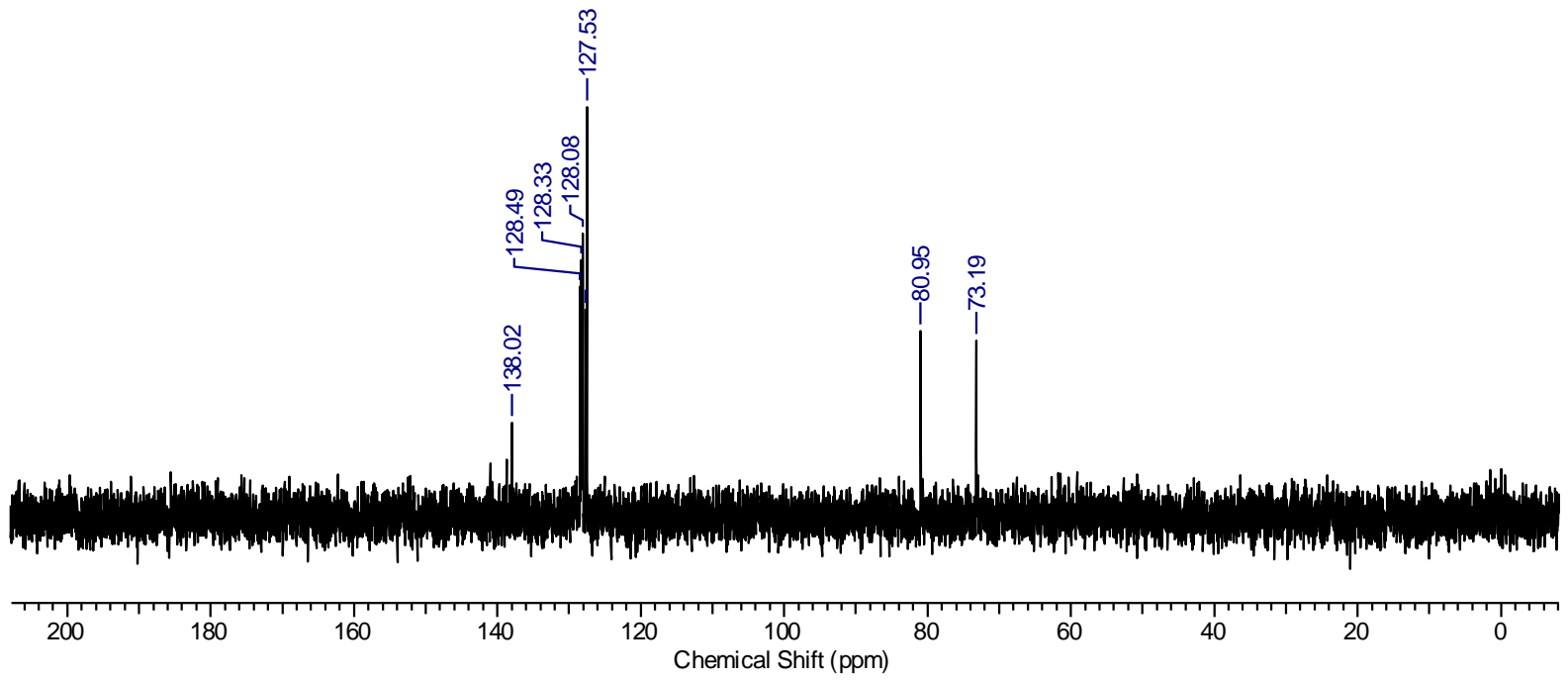


${ }^{1} \mathrm{H},{ }^{13} \mathrm{C}$, DEPT-135 NMR spectra of $2 \mathrm{p}$ :

ID-0715.021.001.1R_FH-153-XY-1H.ESP
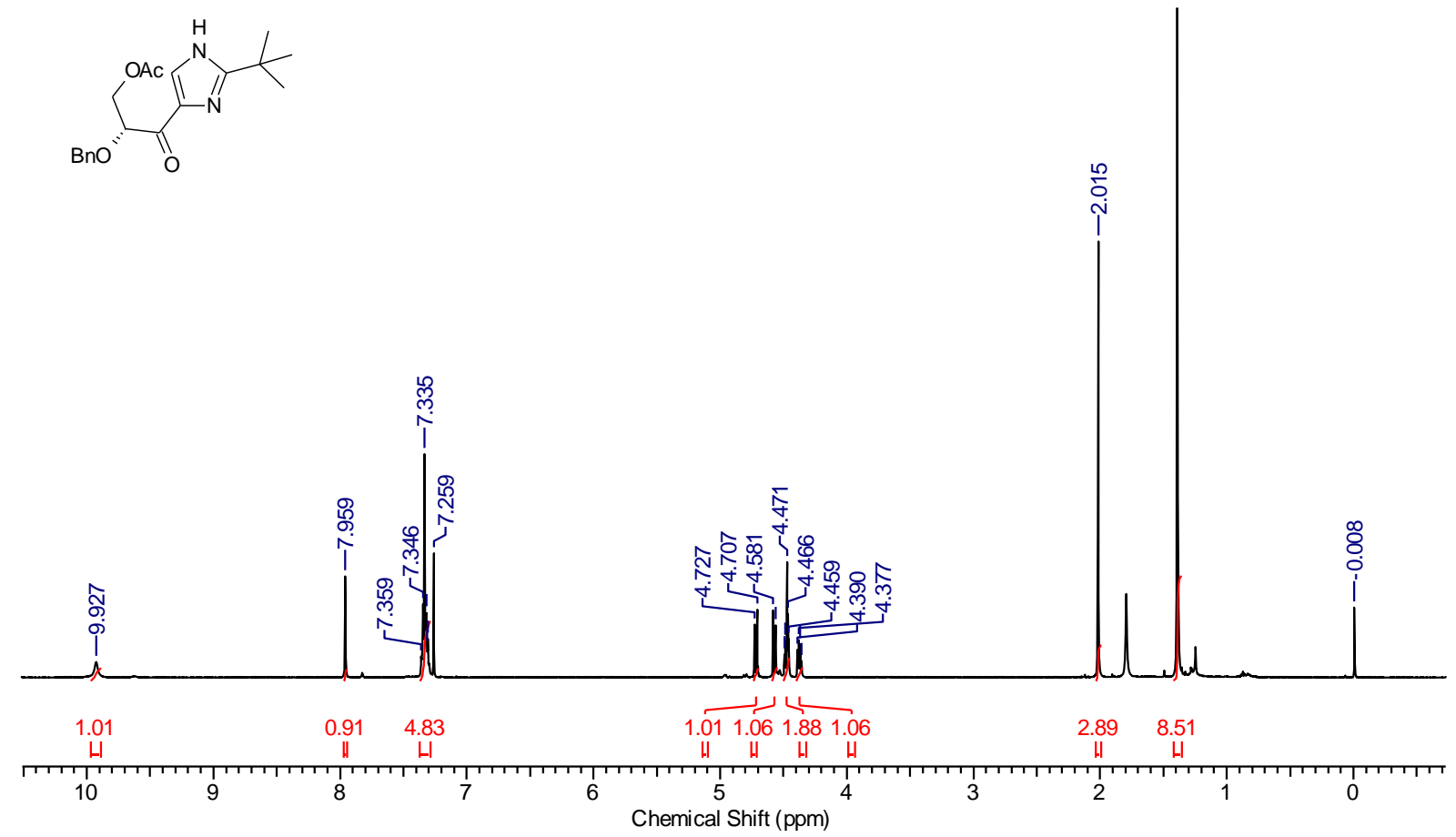

ID-0715.022.001.1R_FH-153-XY-13C.ESP

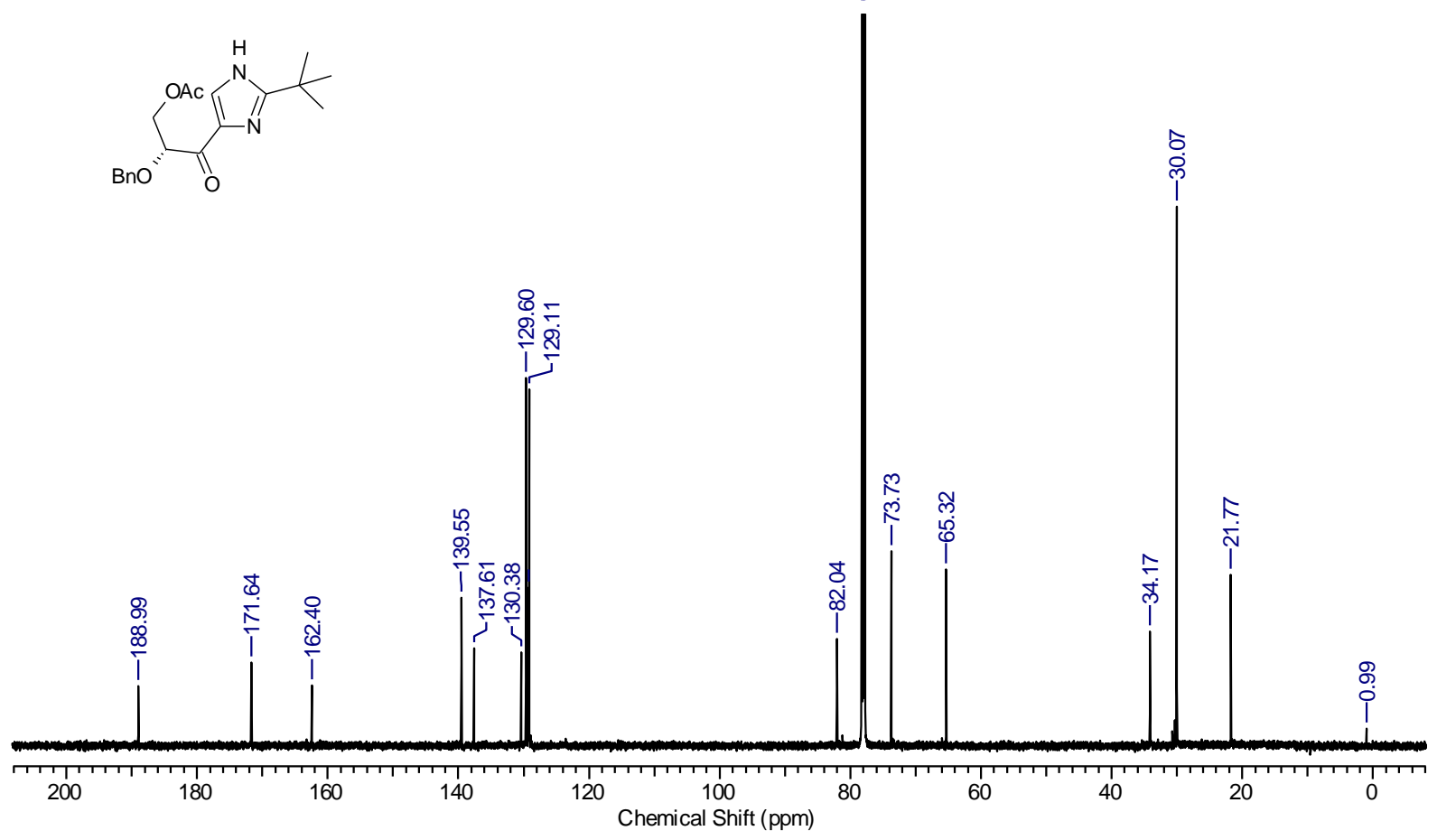



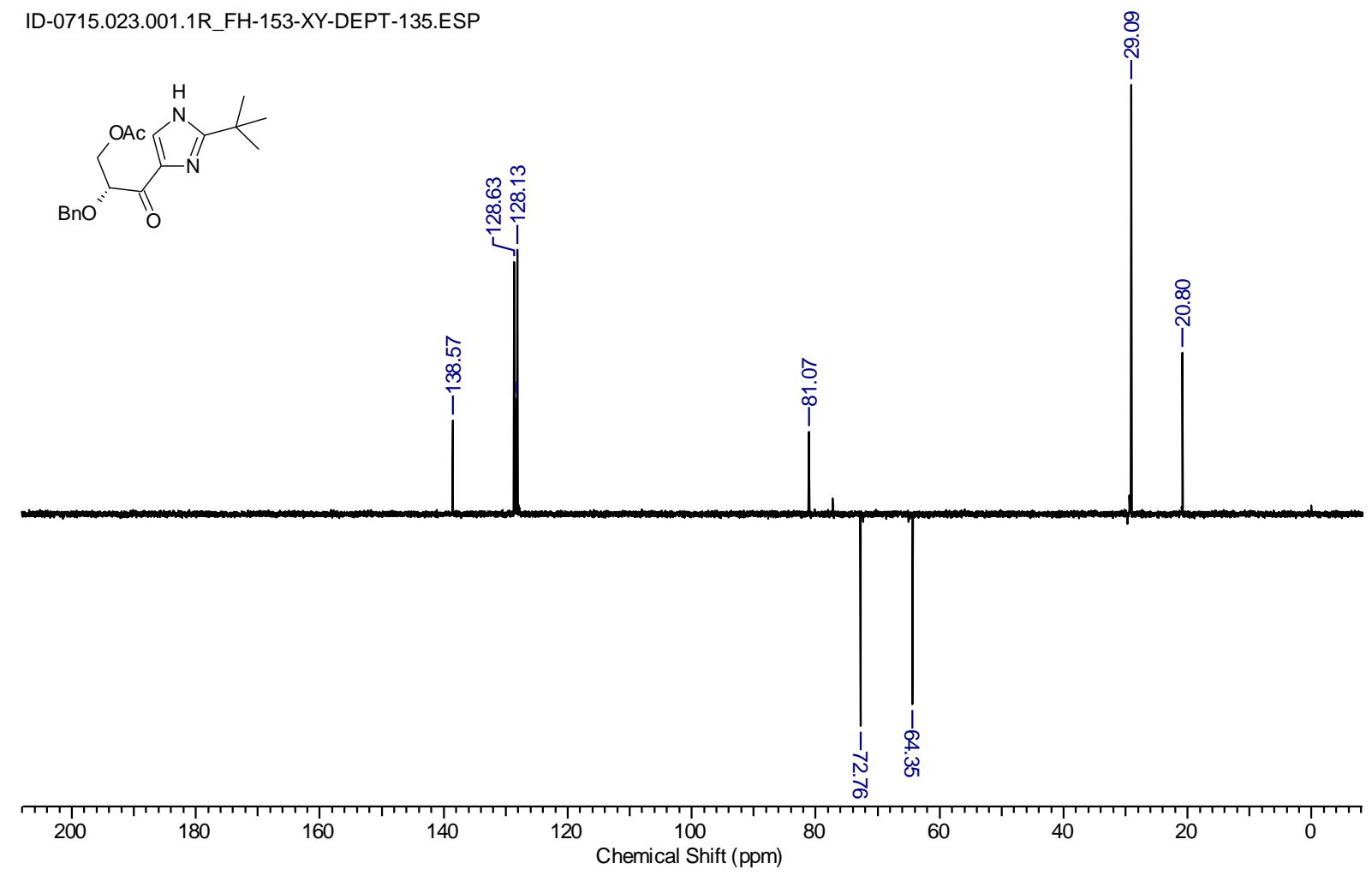

\section{${ }^{1} \mathrm{H},{ }^{13} \mathrm{C}$, DEPT-135, DEPT-90 NMR spectra of 2q:}

ID-0815.001.001.1r.esp_FH-127-1H.esp
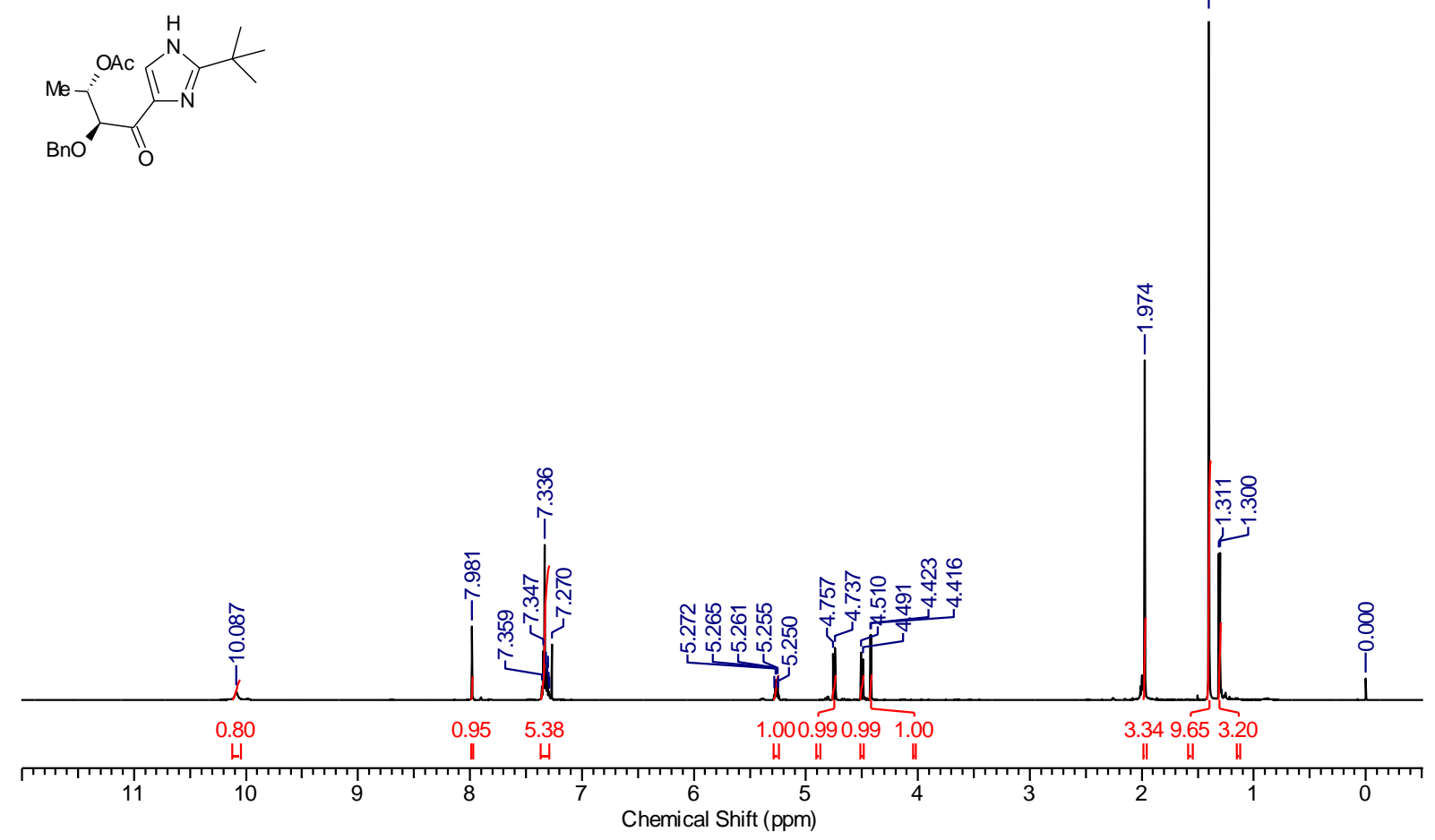


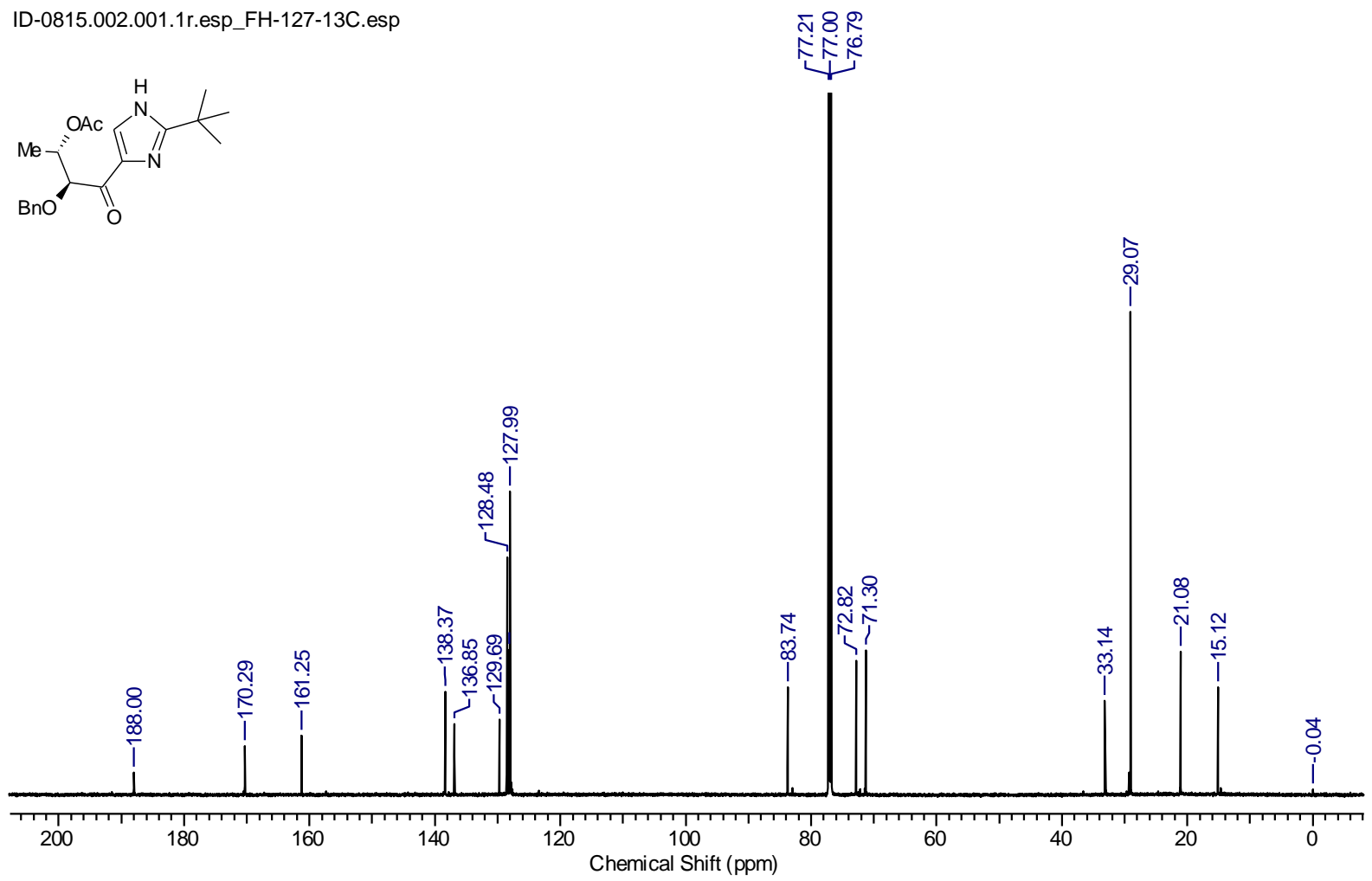

FH-RH-4B-C.002.001.1resp

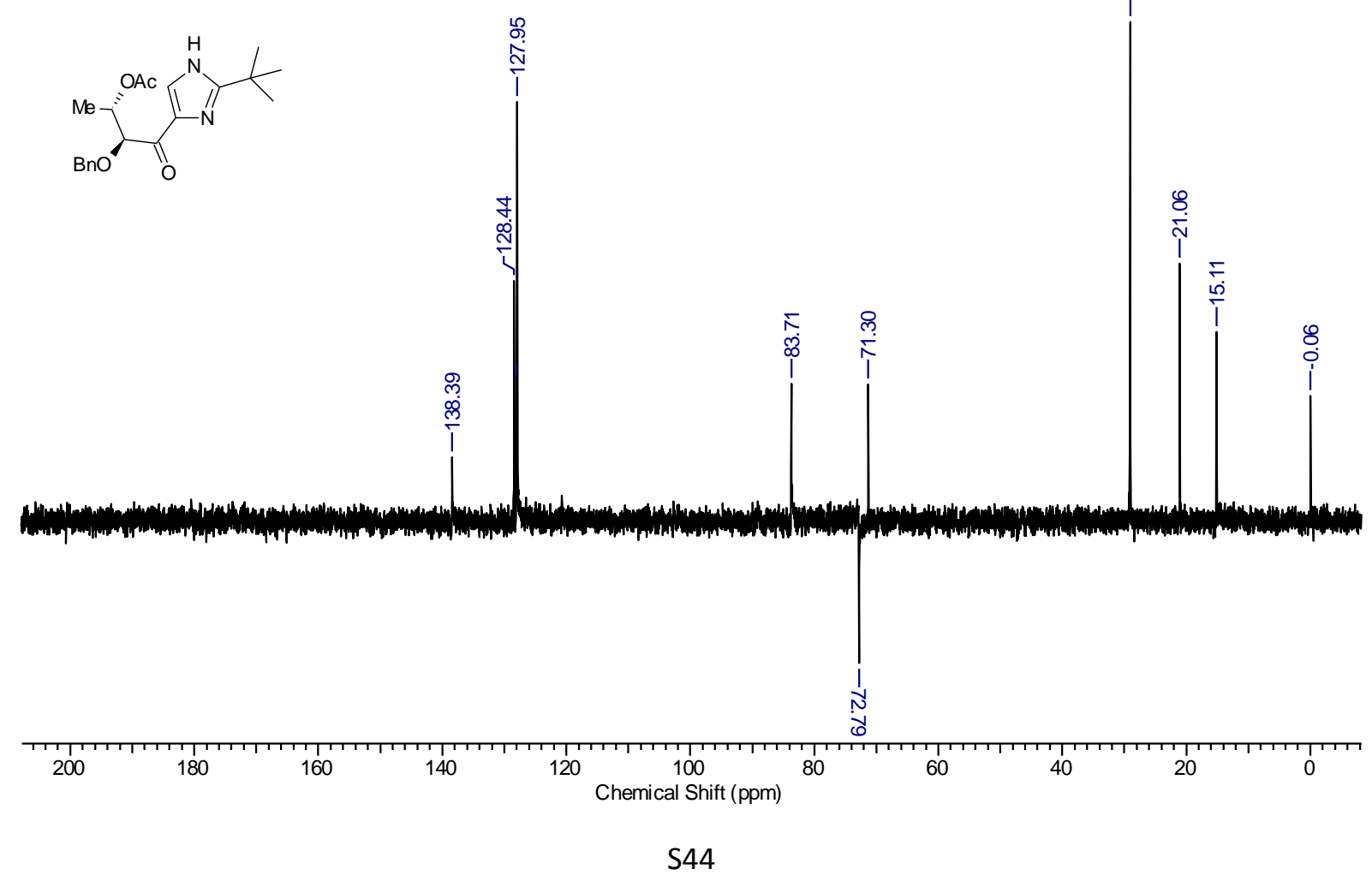


FH-RH-4B-C.003.001.1r.esp<smiles>CC(=O)O[C@@H](C)[C@H](OCc1ccccc1)C(=O)c1c[nH]c(C(C)(C)C)n1</smiles>

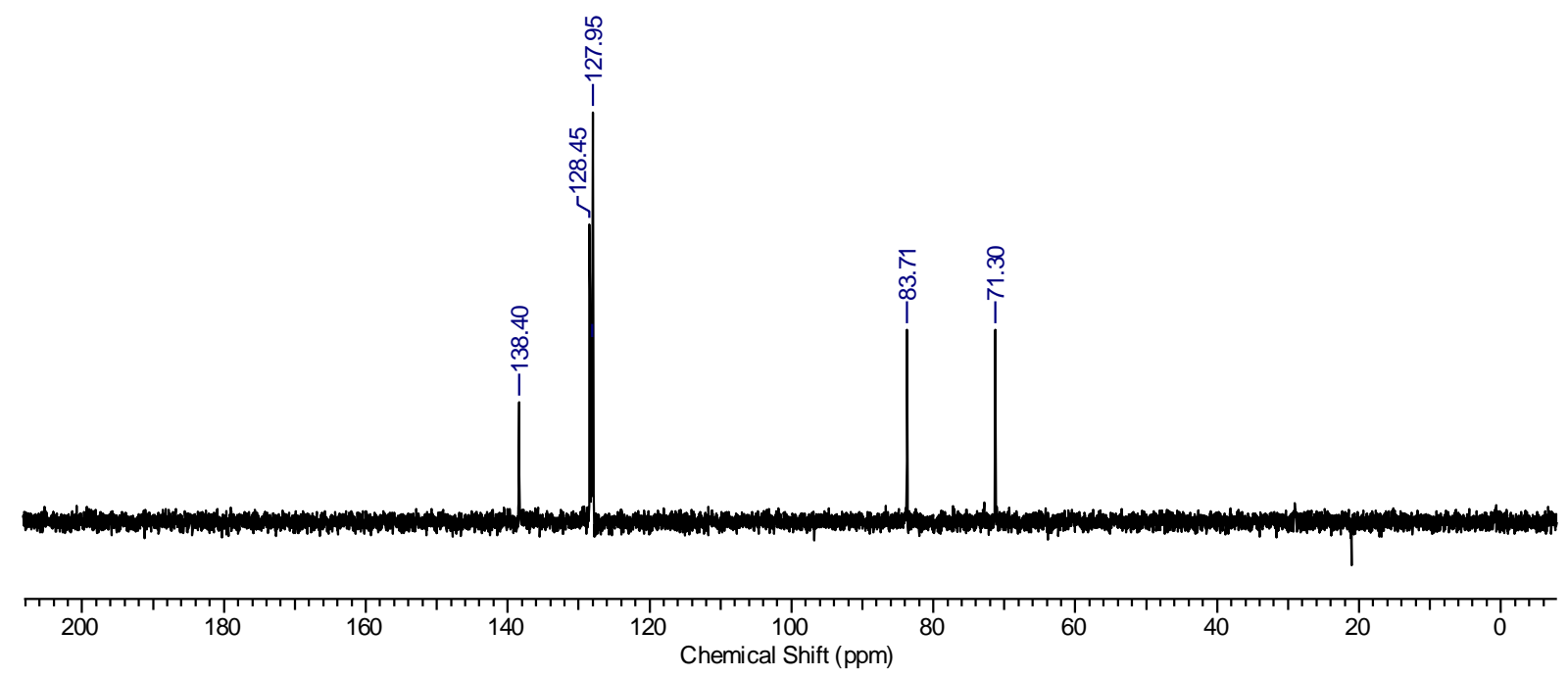

2D (COSY, HMBC, HSQC, H2BC, and NOESY) NMR spectra of 2q:

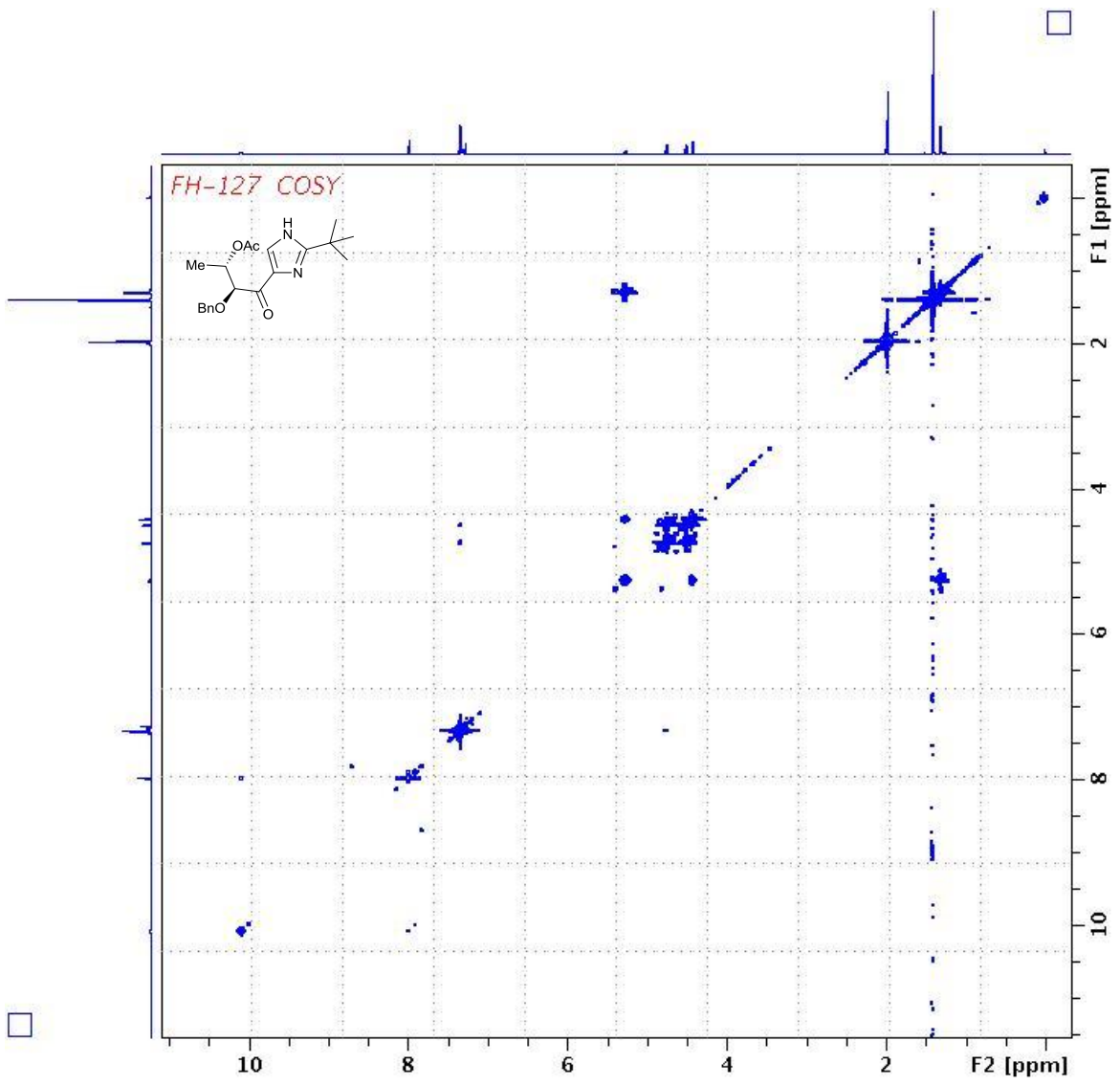



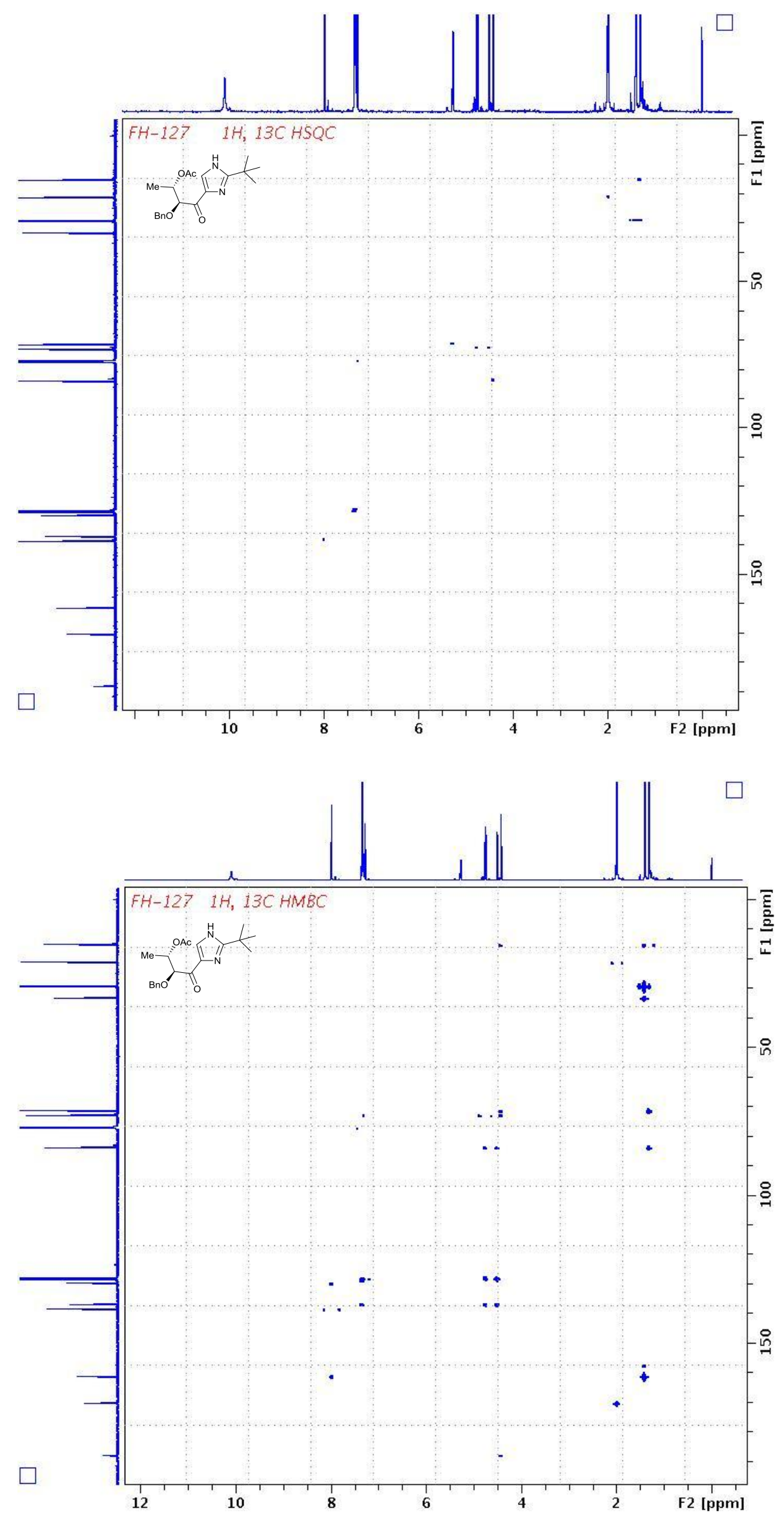

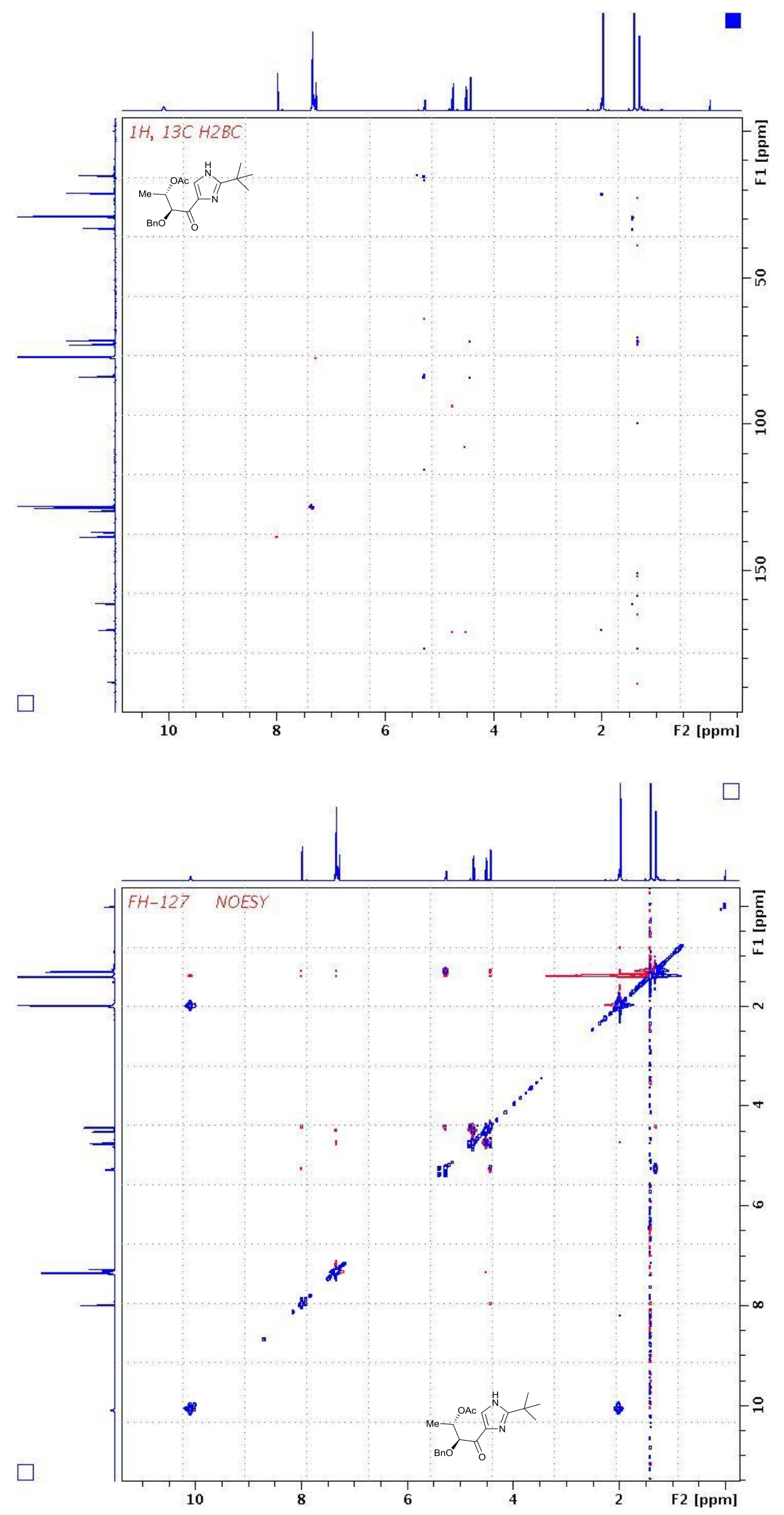
${ }^{1} \mathrm{H},{ }^{13} \mathrm{C}$, DEPT-135, DEPT-90 NMR spectra of 2r:

FH-FU-4A-1H.001.001.1r.esp
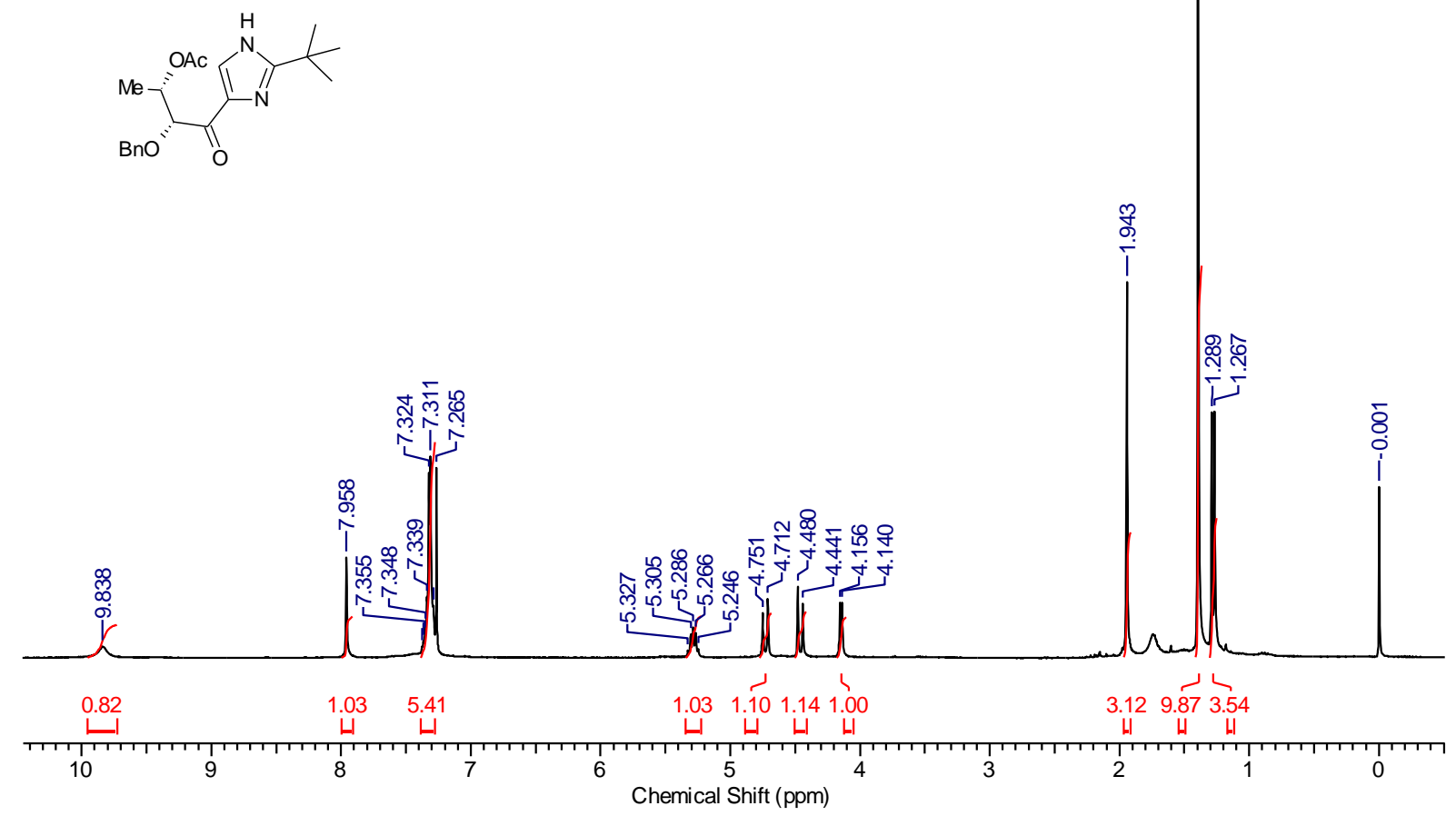

FH-131-C.001.001.1r.esp
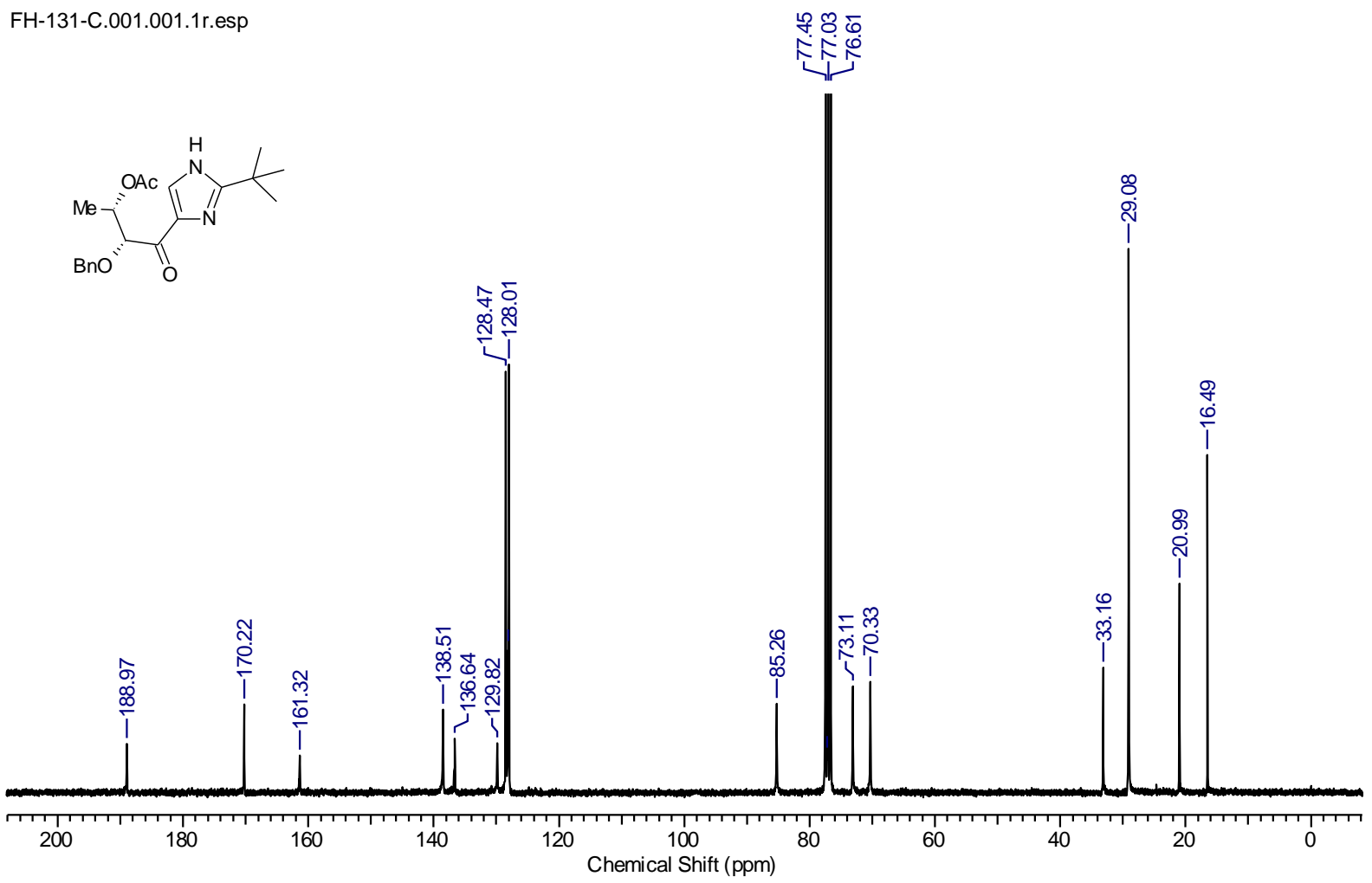

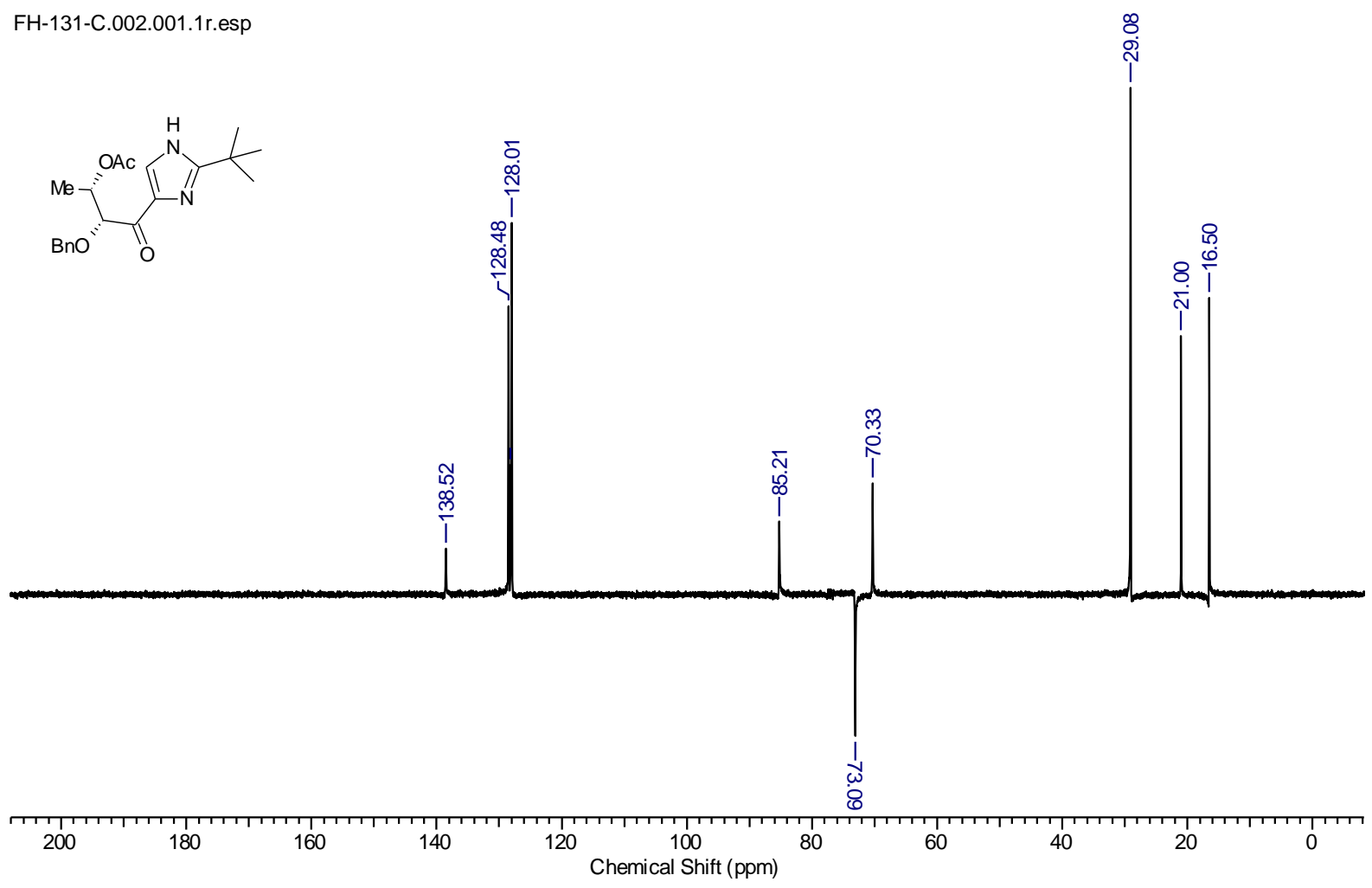

FH-131-C.003.001.1r.esp<smiles>CC(=O)O[C@@H](Cc1ccccc1)C(C(=O)O)C(=O)O</smiles>

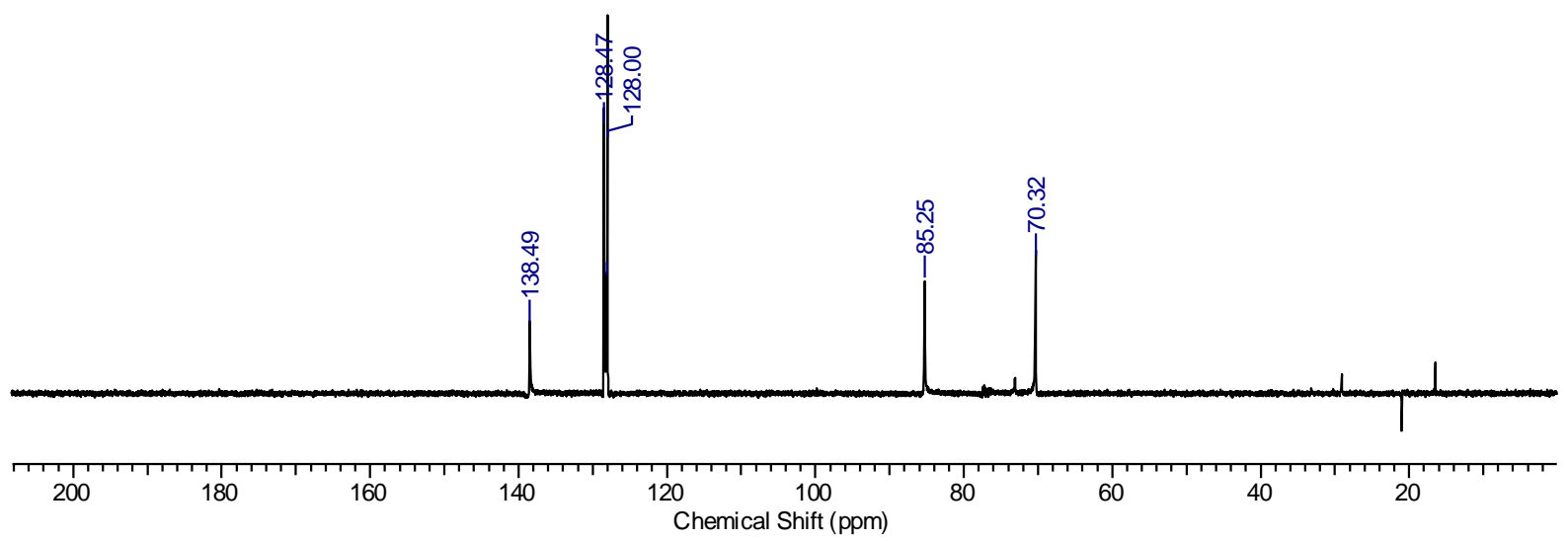


${ }^{1} \mathrm{H},{ }^{13} \mathrm{C}$, DEPT-135, DEPT-90 NMR spectra of 2s:

FH-GA-4B.029.001.1r.esp
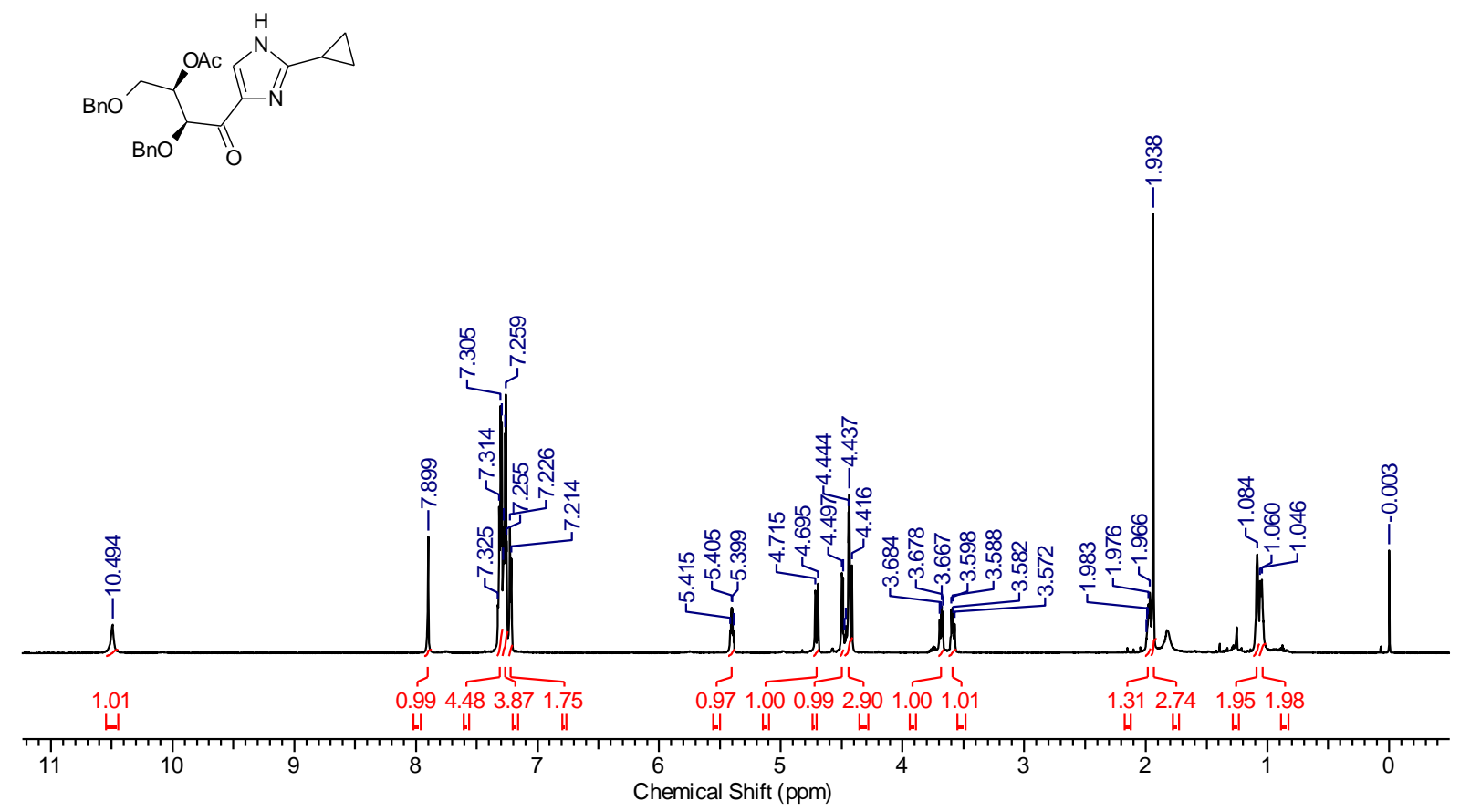

FH-GA-4B.030.001.1R.ESP<smiles>CC(C)O[C@@H](COCc1ccccc1)[C@@H](O)C(=O)c1c[nH]c(C2CC2)n1</smiles>

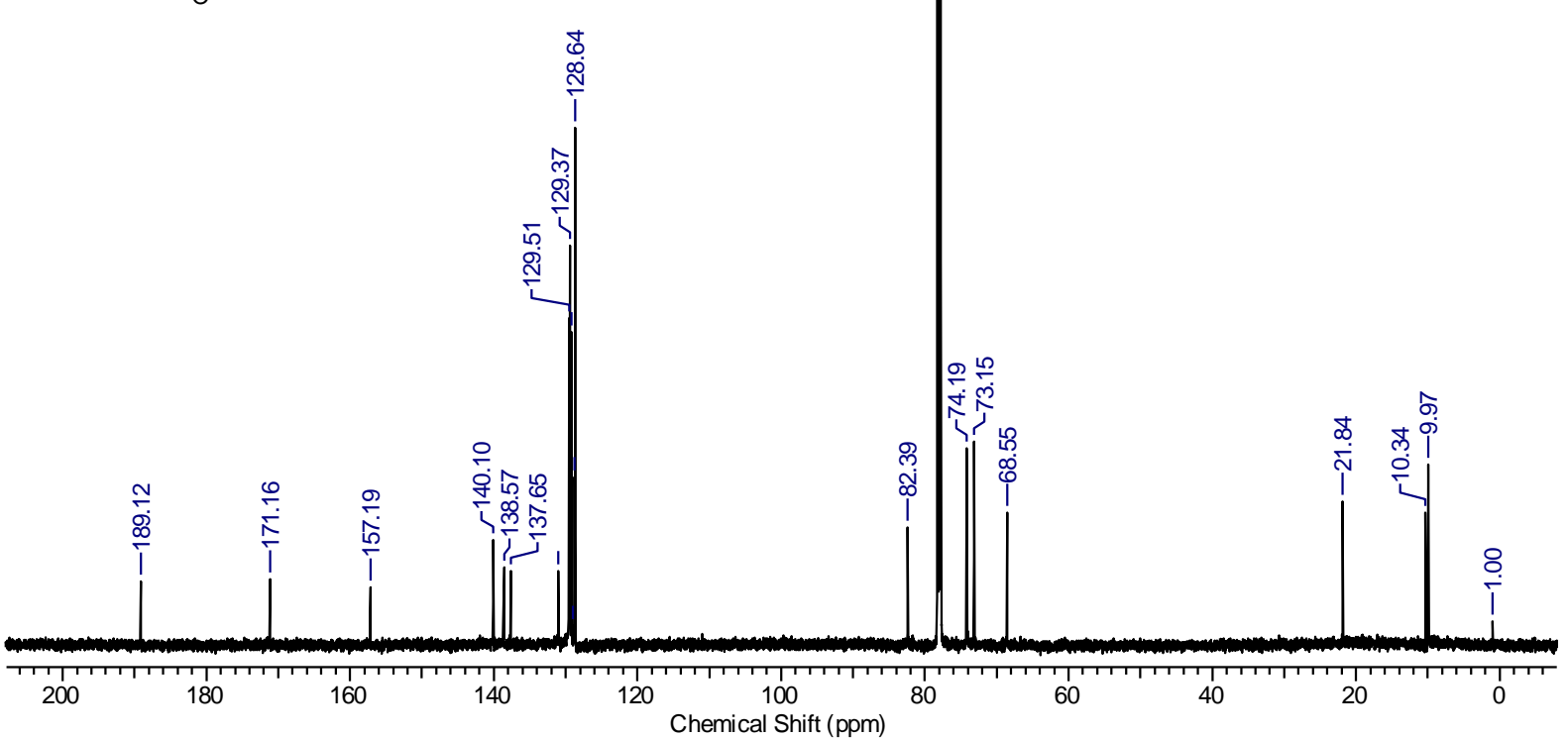




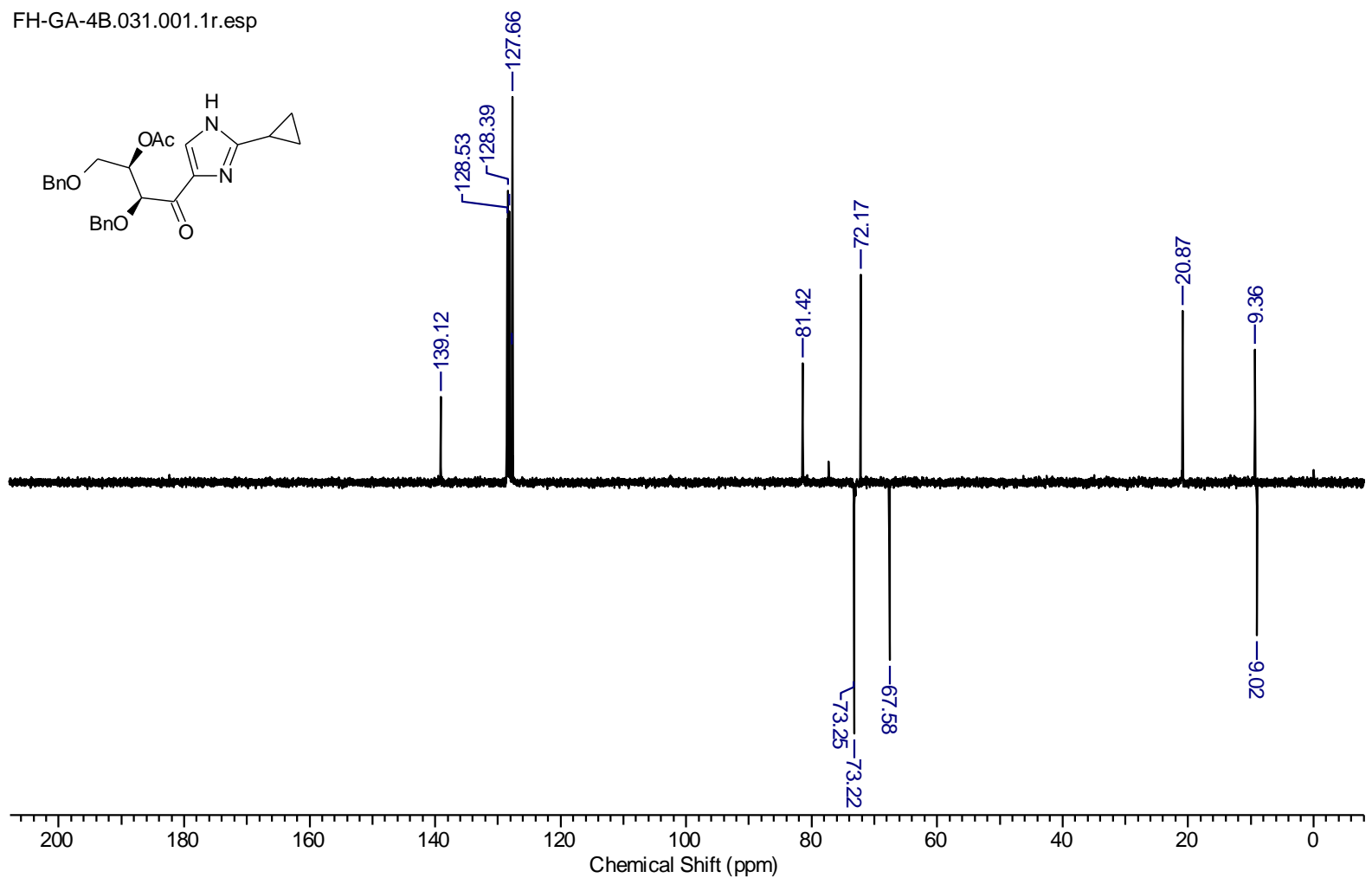

FH-GA-4B.032.001.1r.esp<smiles>O=C(c1ccccc1)c1nc(C2CC2)[nH]c1C(=O)C(O)COCc1ccccc1</smiles>

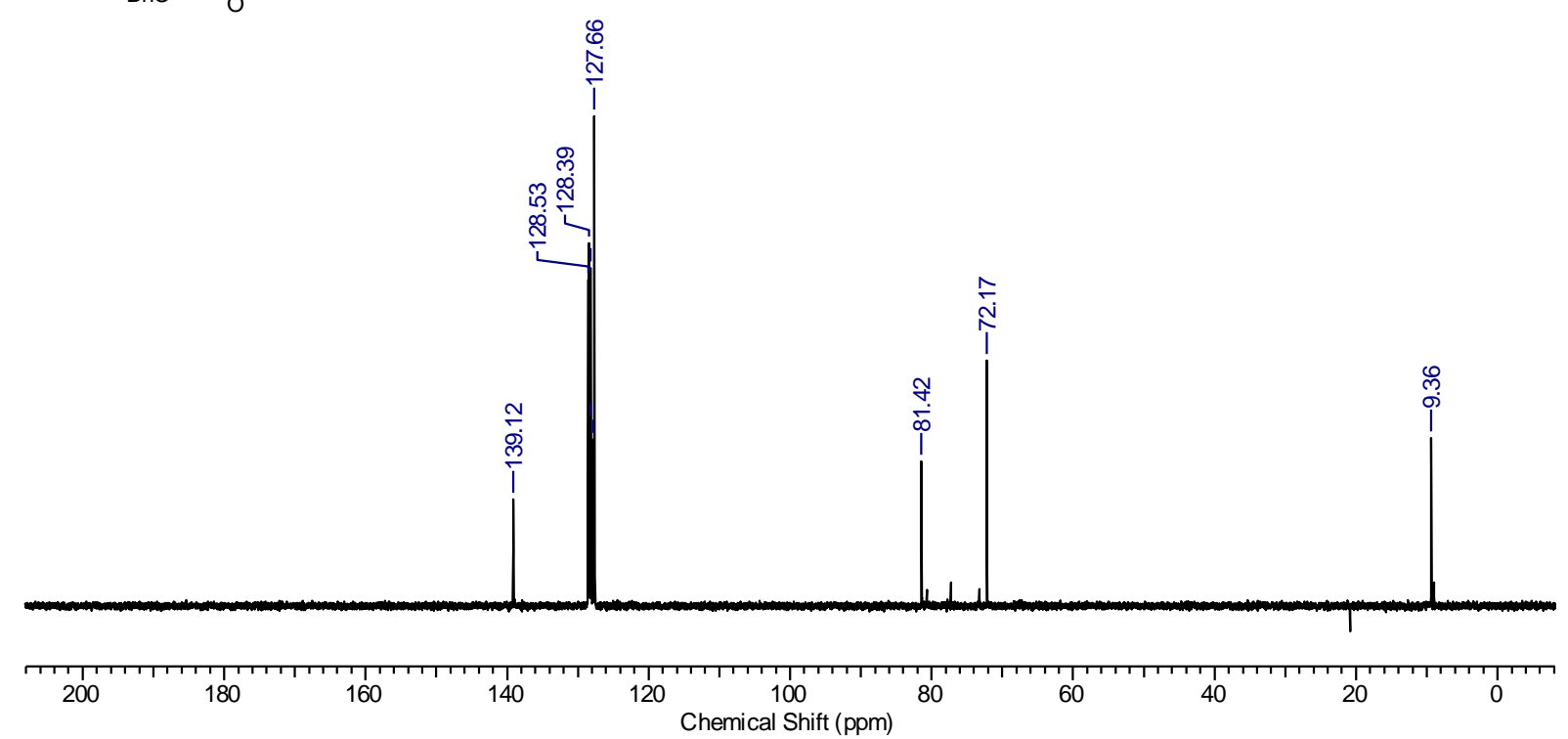


${ }^{1} \mathrm{H},{ }^{13} \mathrm{C}$, DEPT-135, DEPT-90 NMR spectra of 2t:

FH-GL-3A-1H.001.001.1r.esp<smiles>O=C(COCc1ccccc1)O[C@H](C(=O)c1c[nH]c(C2CC2)n1)[C@H](O)Cc1ccccc1</smiles>

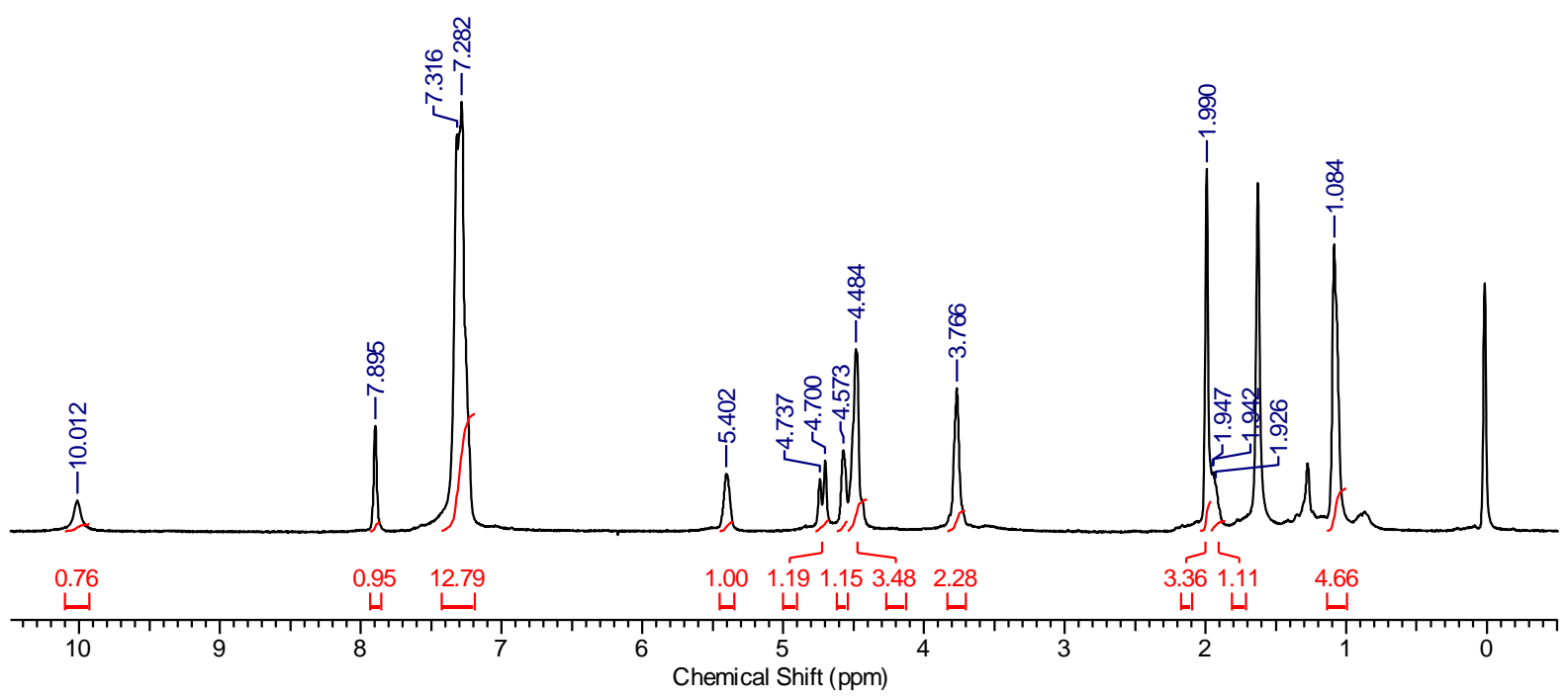

FH-GL-3B-C.001.001.1R.ESP

ษ 8

손<smiles>O=C(C(=O)C(Cc1ccccc1)OCc1ccccc1)c1c[nH]c(C2CC2)n1</smiles>

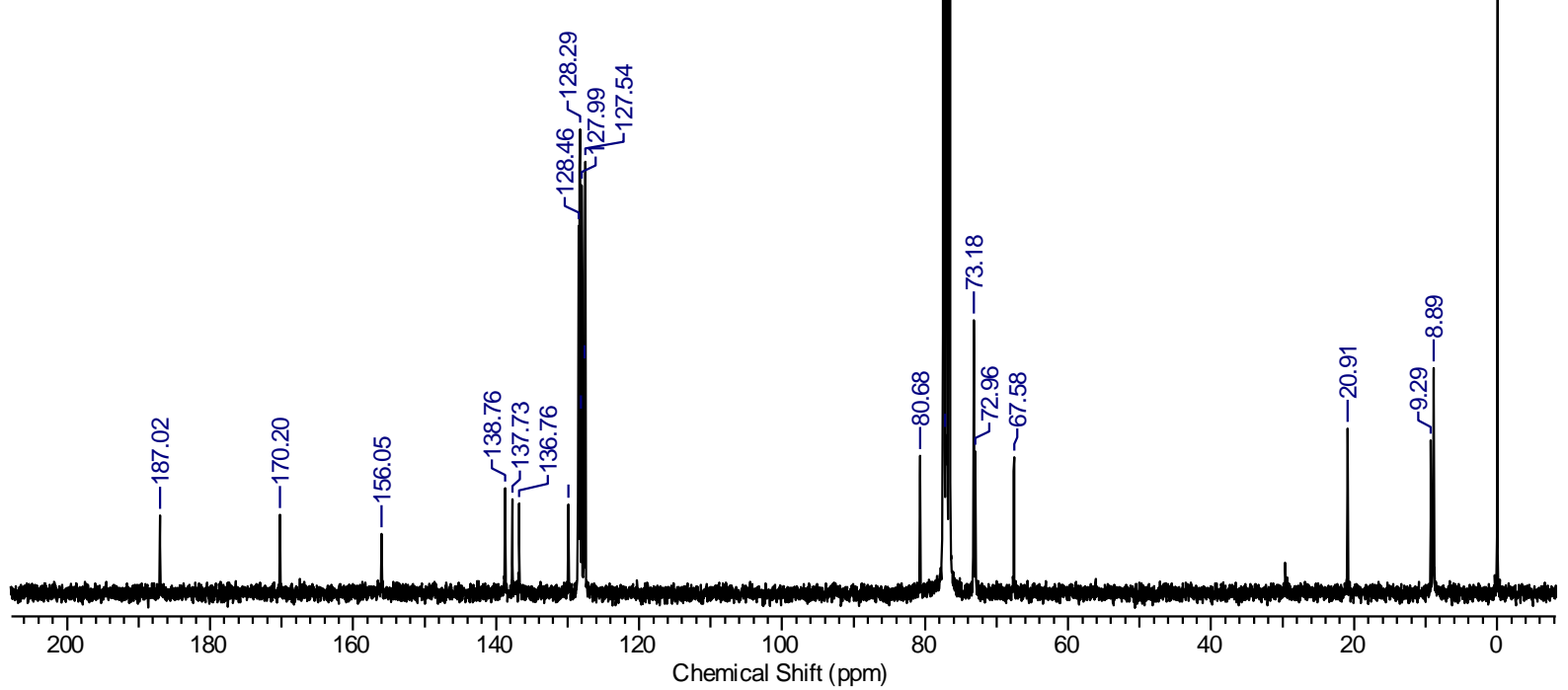


FH-GL-3B-C.002.001.1R.ESP

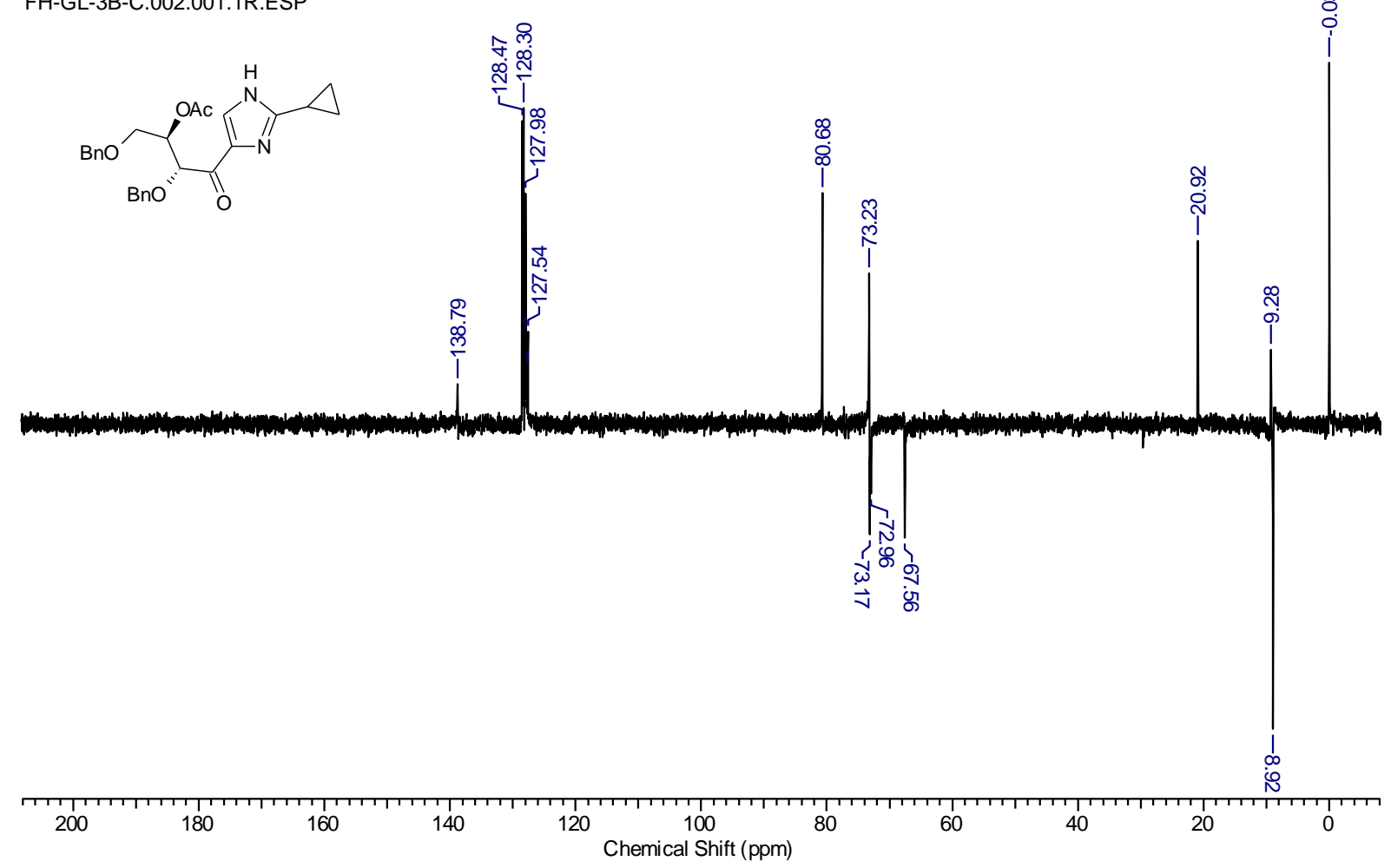

FH-GL-3B-C.003.001.1r.esp
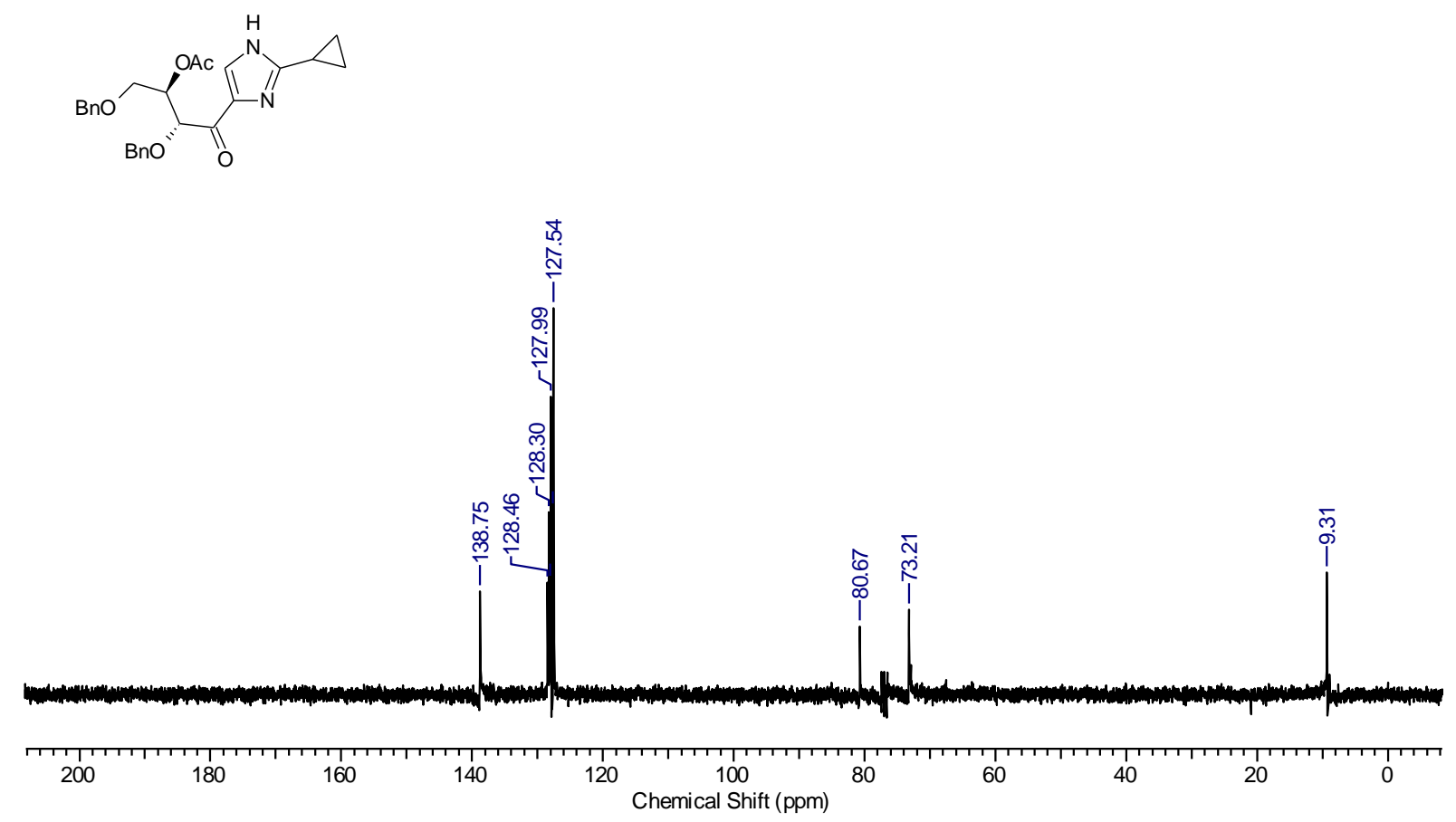


\section{${ }^{1} \mathrm{H},{ }^{13} \mathrm{C}$, DEPT-135, DEPT-90 NMR spectra of 2u:}

FH-RH-3A-1H.001.001.1r.esp

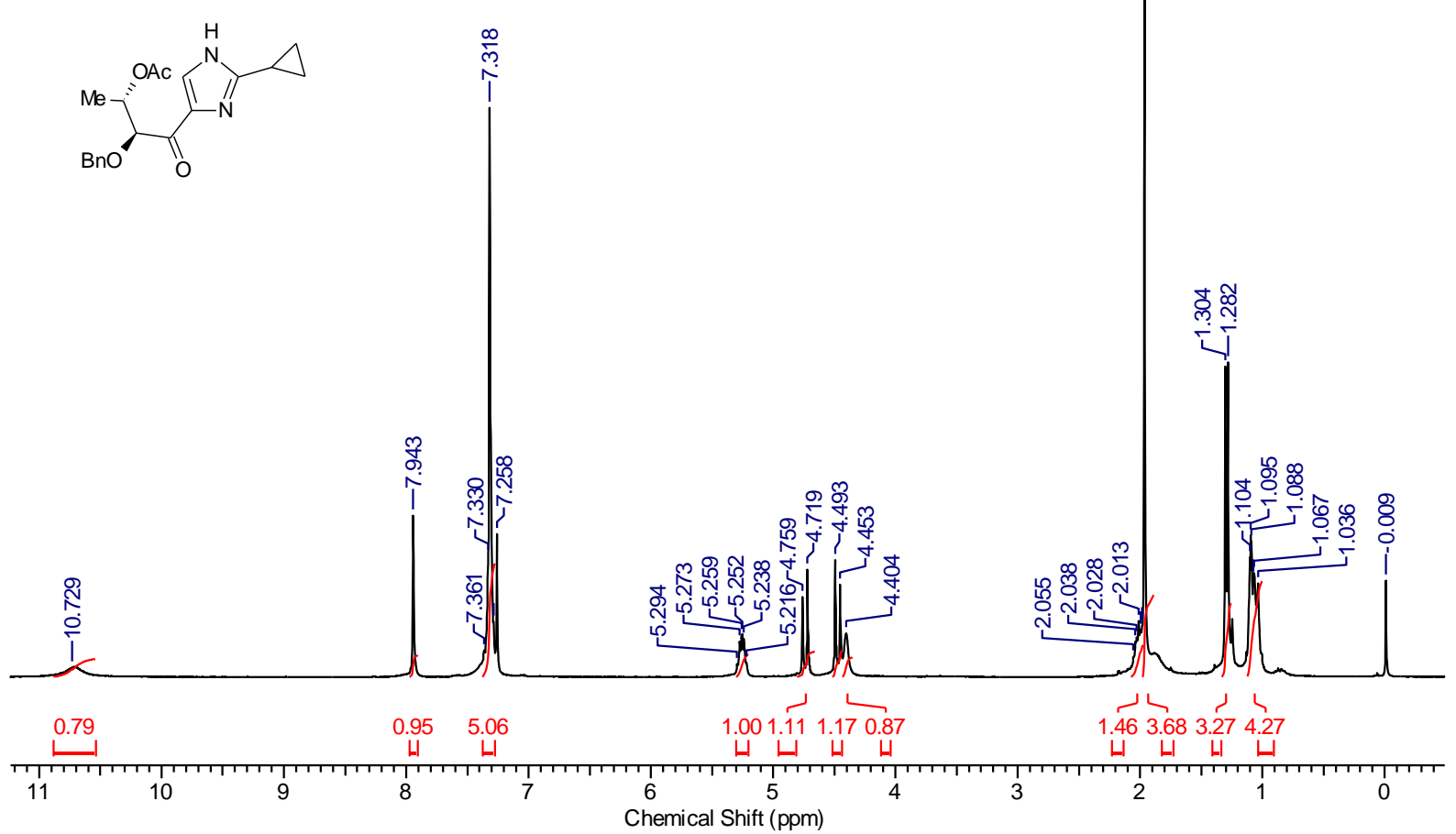

ID-0615.010.001.1R_FH-RH-3A-13C.ESP
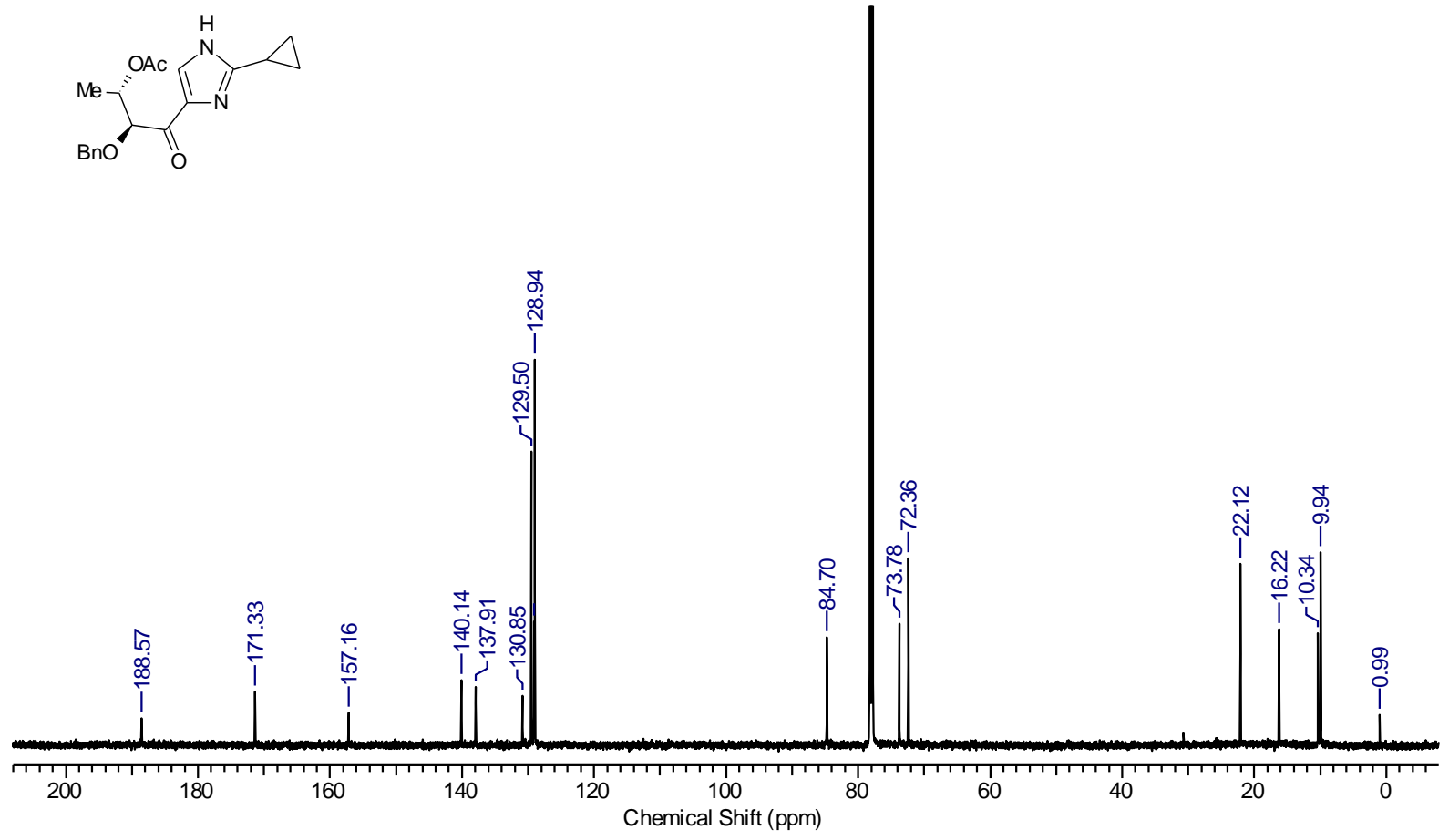


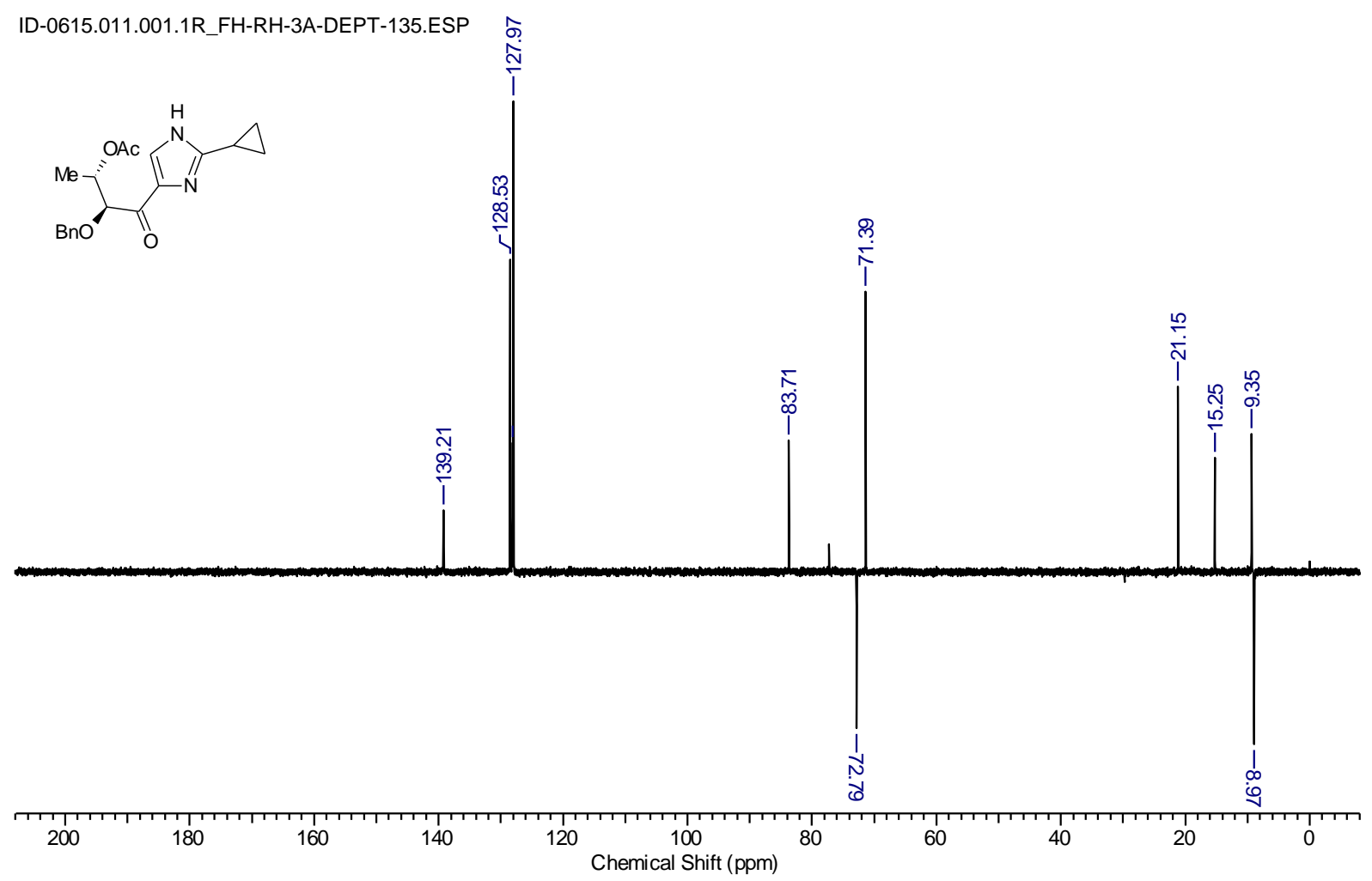

ID-0615.012.001.1R_FH-RH-3A-DEPT-90.ESP
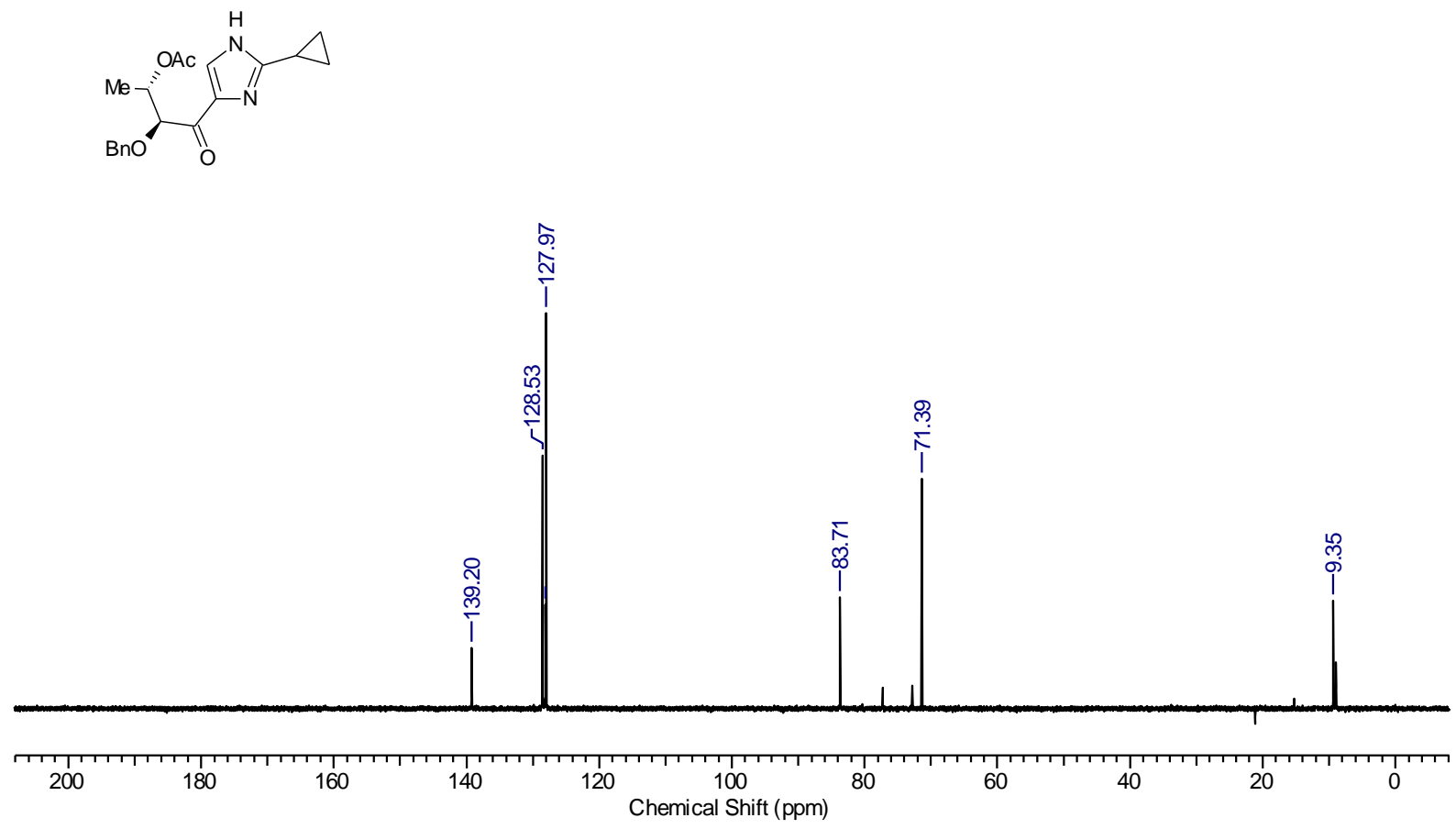

S55 
${ }^{1} \mathrm{H},{ }^{13} \mathrm{C}$, DEPT-135, DEPT-90 NMR spectra of 2v:

FH-FU-3A-1H.001.001.1r.esp
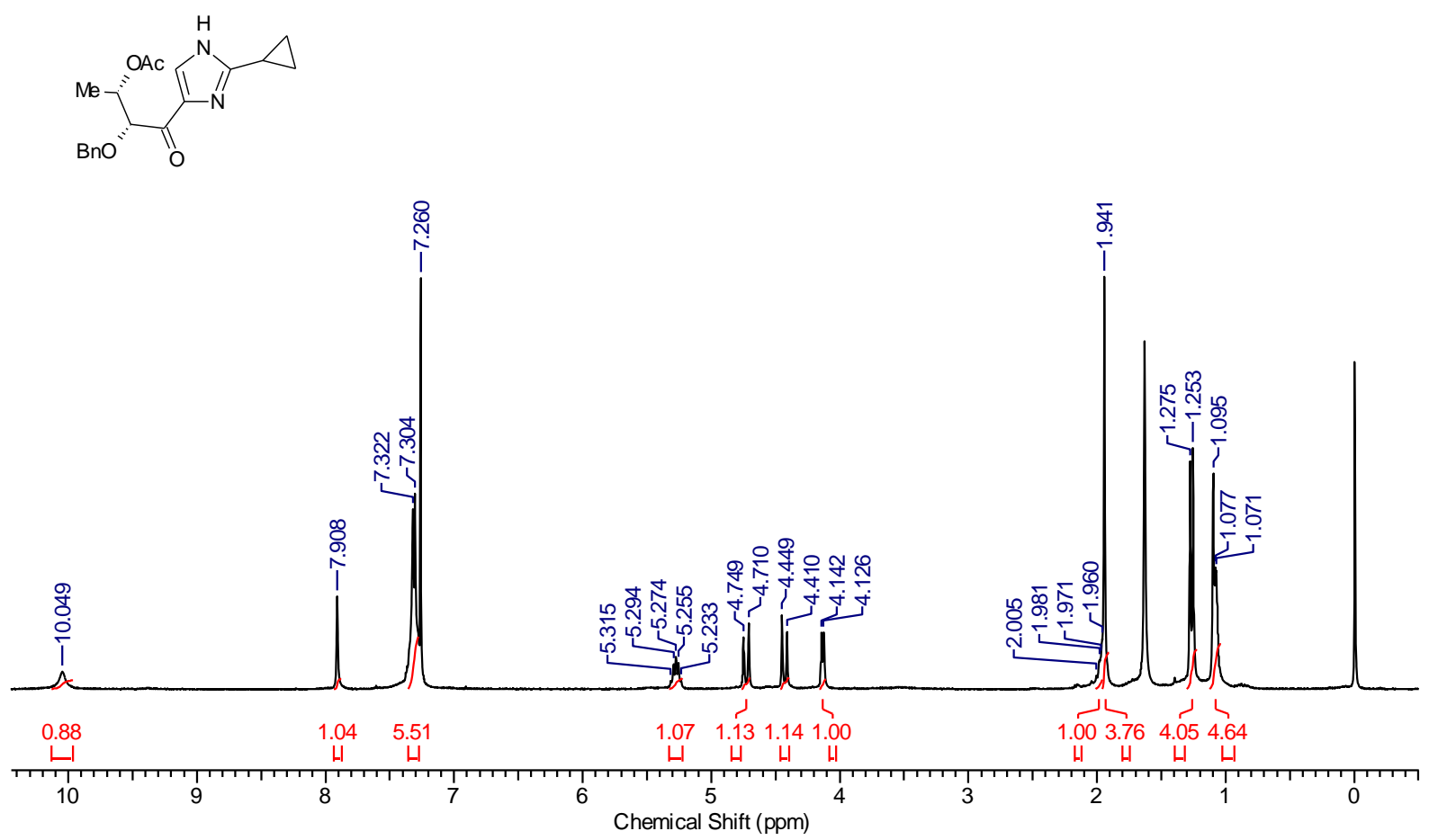

FH-FU-3B-C.001.001.1r.esp
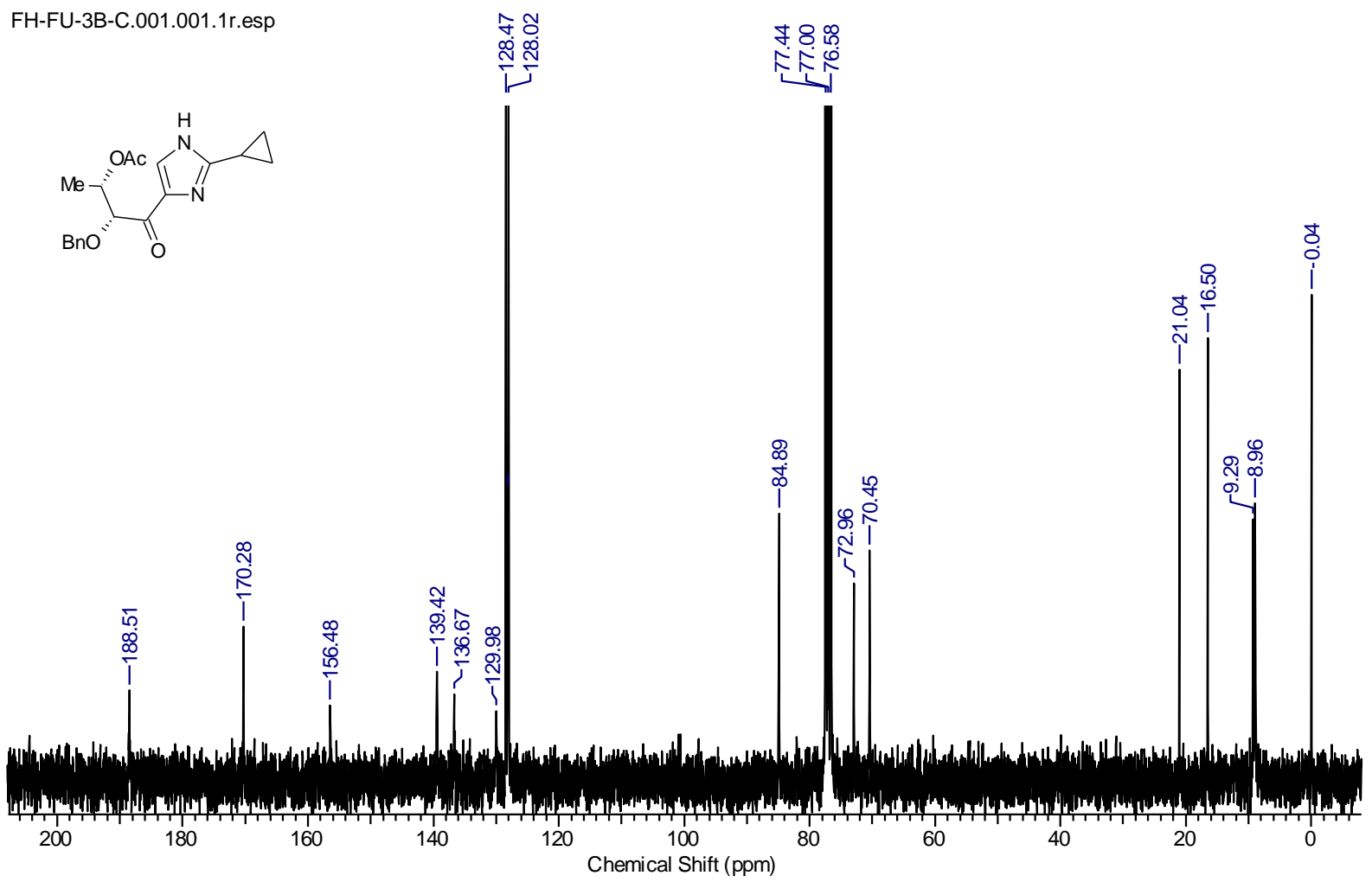


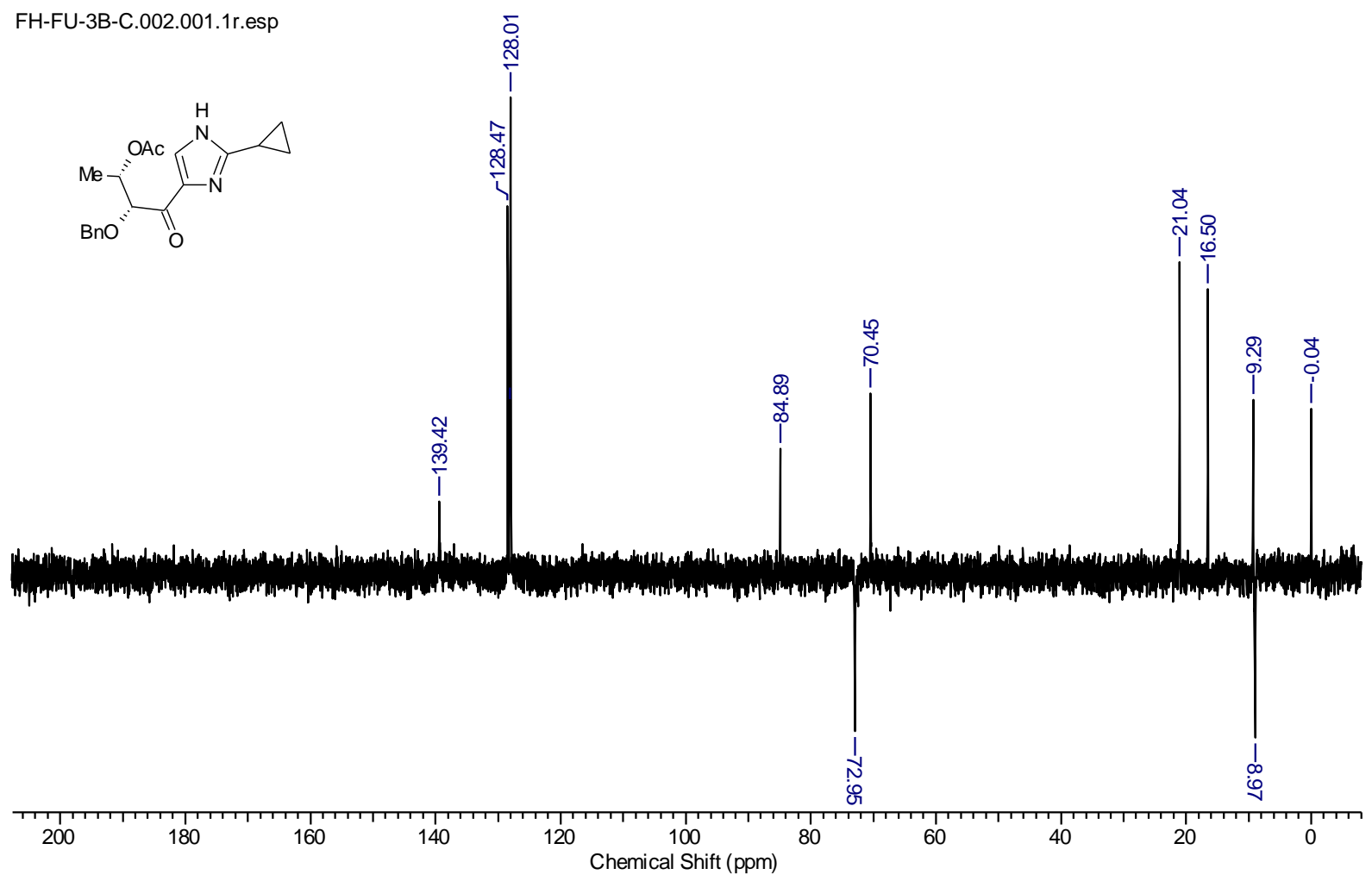

FH-FU-3B-C.003.001.1r.esp

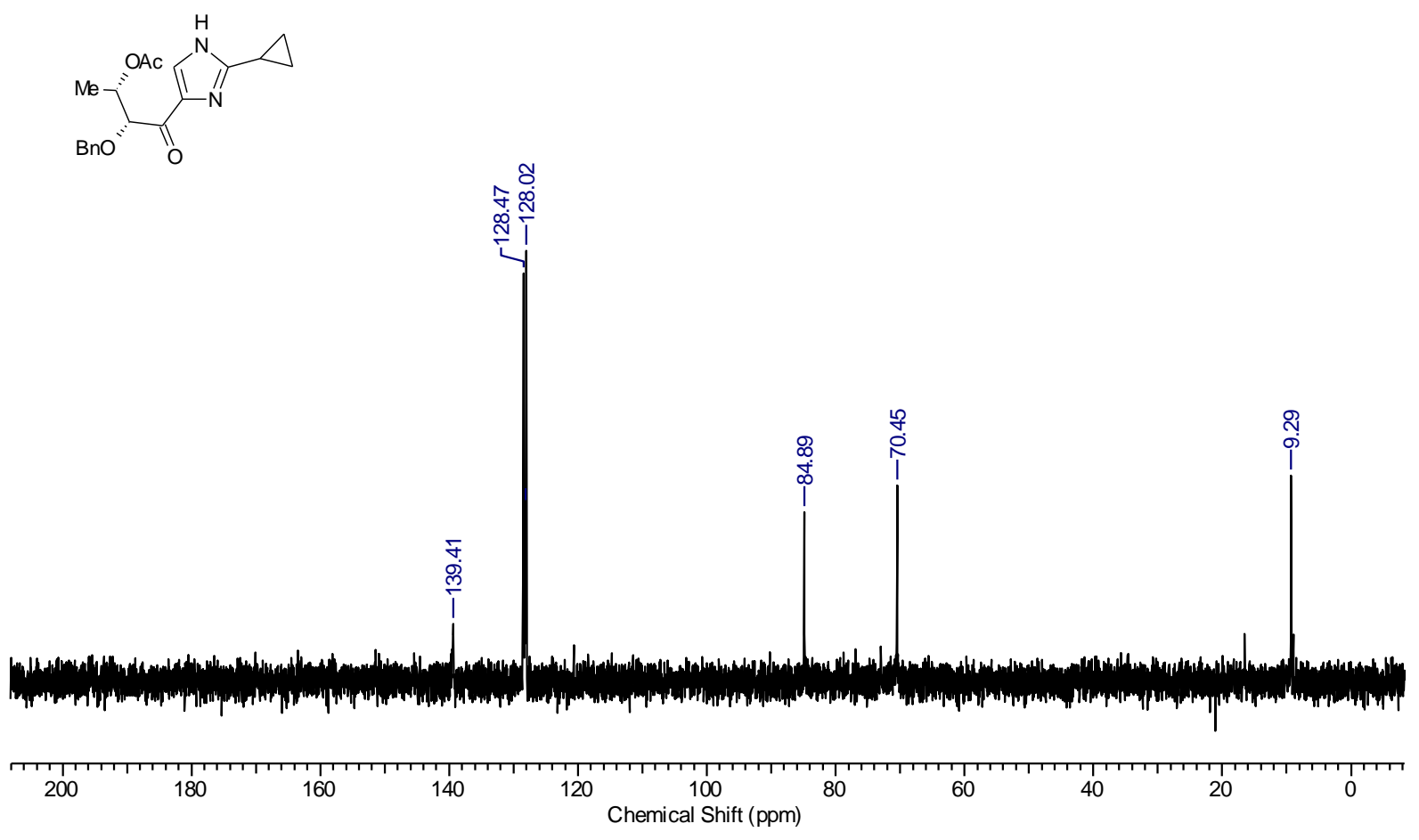




\section{${ }^{1} \mathrm{H},{ }^{13} \mathrm{C}$, DEPT-135 NMR spectra of $2 \mathrm{w}$ :}

NMR FILES_HAQUE.041.001.1R_FH-158-1H.ESP<smiles>O=C(c1nc(-c2ccccc2)[nH]c1I)[C@H](O)[C@@H](O)COc1ccccc1</smiles>
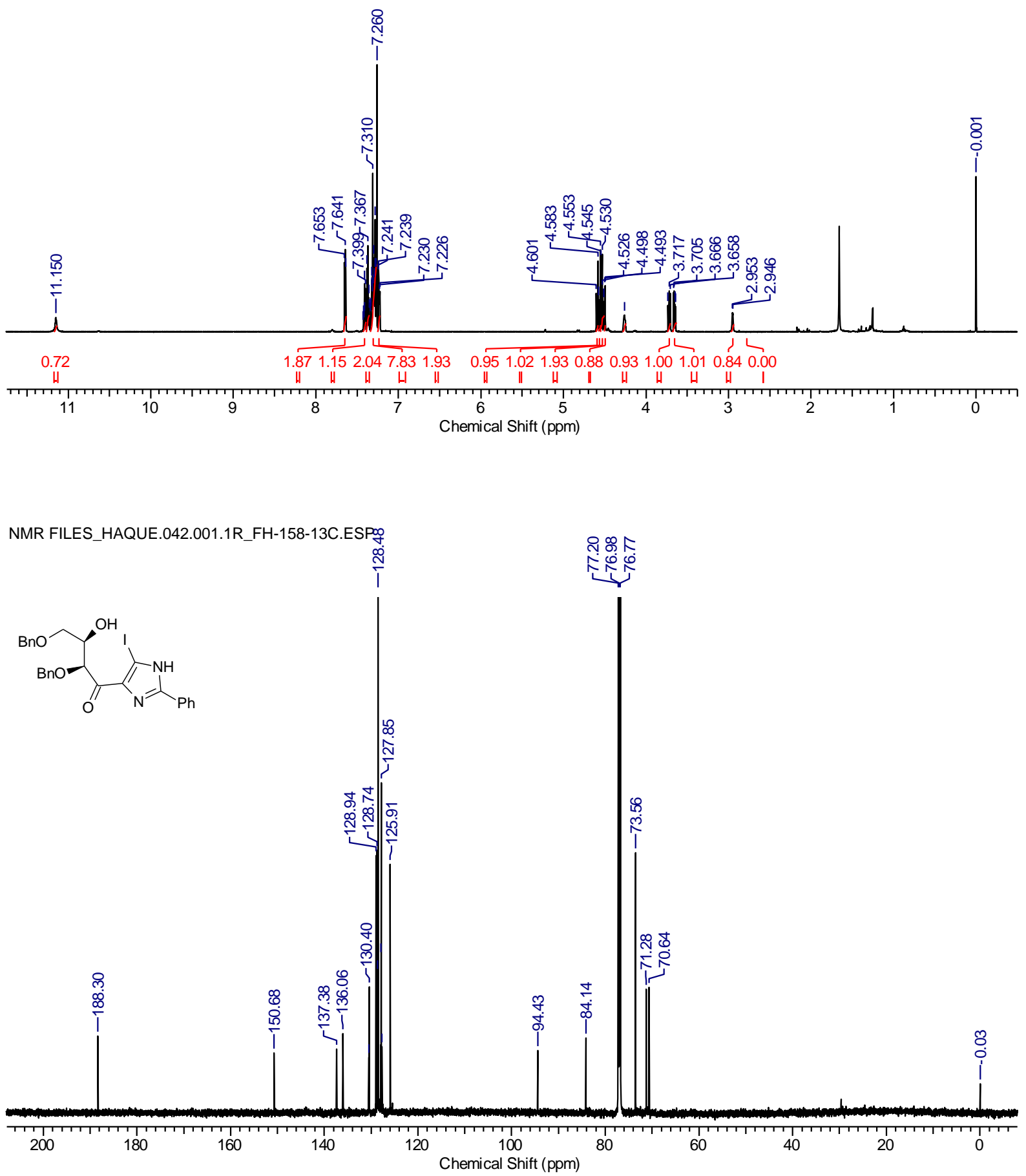


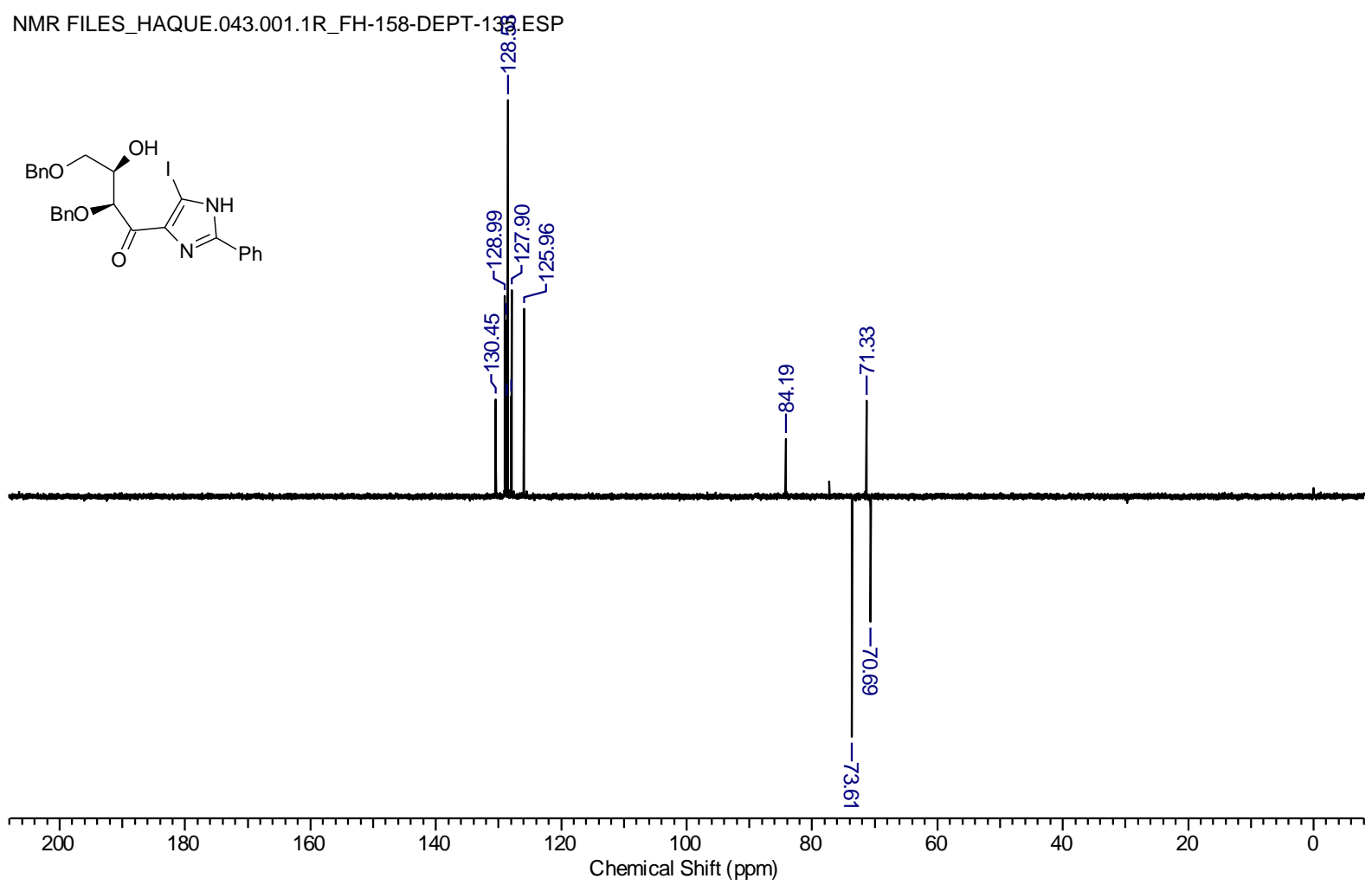

${ }^{1} \mathrm{H},{ }^{13} \mathrm{C}$, DEPT-135 NMR spectra of $2 \mathrm{x}$ :

081.001.1r_FH-180-1H.esp<smiles>O=C(c1nc(-c2ccccc2)[nH]c1I)[C@H](O)[C@H](O)COCc1ccccc1</smiles>

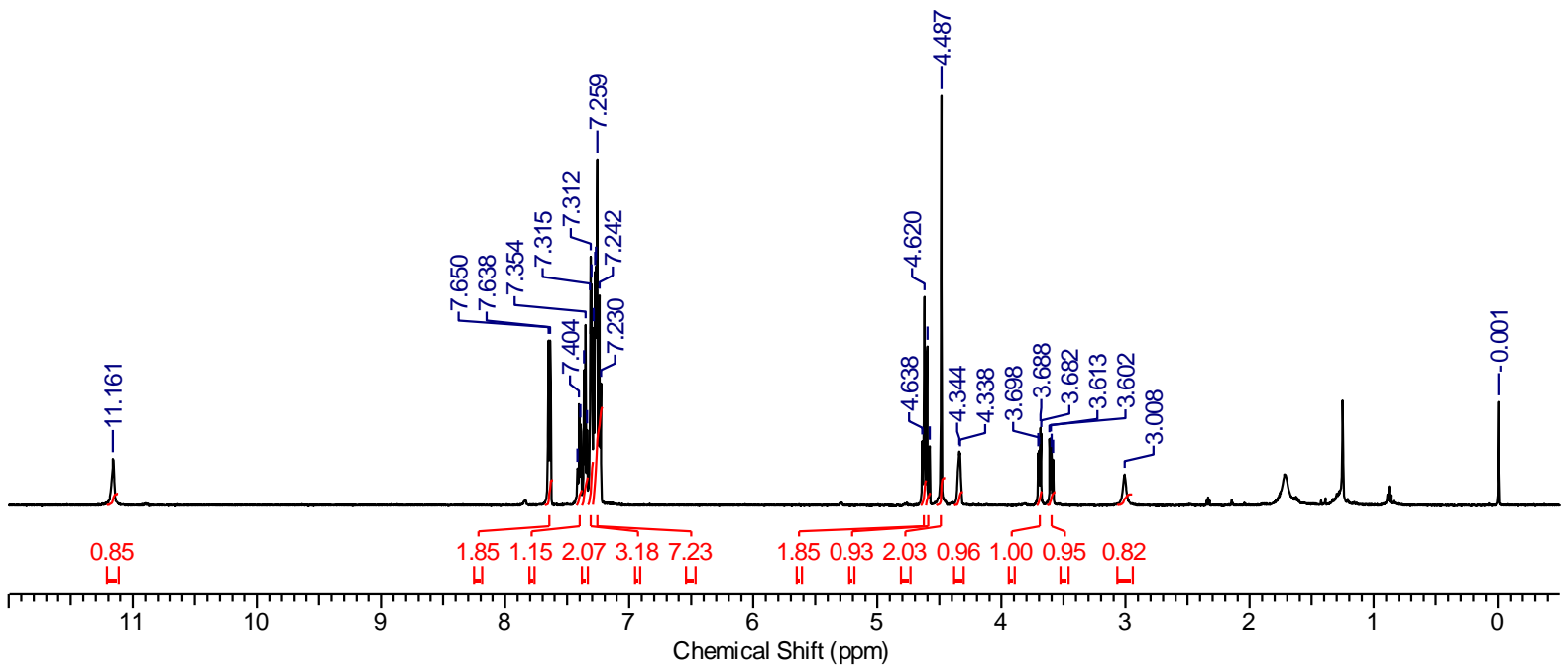


082.001.1r_FH-180-13C.esp<smiles>O=C(c1nc(-c2ccccc2)[nH]c1I)[C@H](O)[C@H](O)COc1ccccc1</smiles>

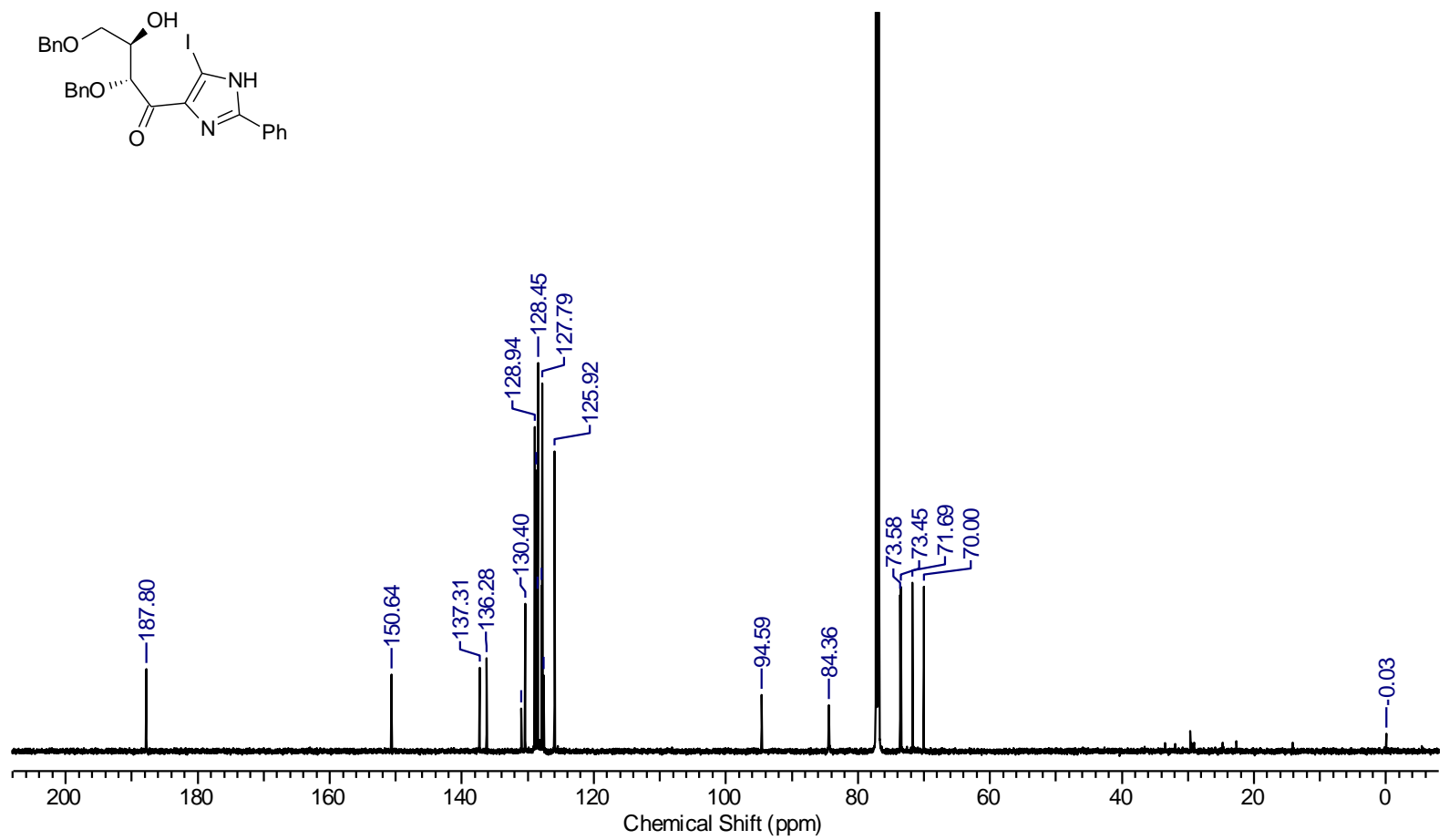

.083.001.1r FH-180-DEPT-135.esp<smiles>O=C(c1nc(-c2ccccc2)[nH]c1I)[C@H](O)[C@H](O)COCc1ccccc1</smiles> 


\section{${ }^{1} \mathrm{H},{ }^{13} \mathrm{C}$, DEPT-135 NMR spectra of 3a:}

ID-0615.055.001.1r_KM-443-1H.esp<smiles>CCOC(=O)c1cc(C(=O)[C@@H](Br)[C@H](C)O)oc1C</smiles>
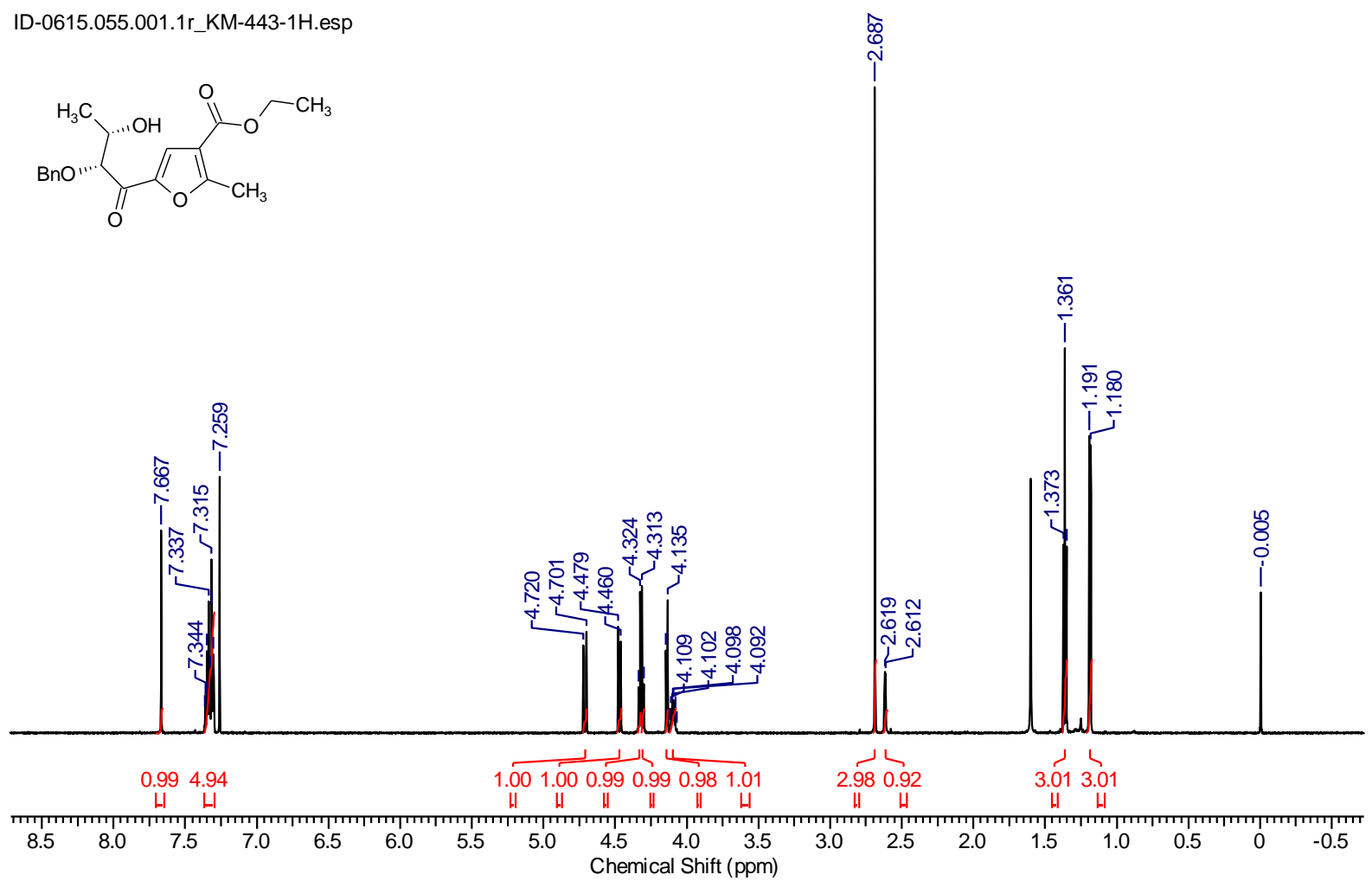

ID-0615.056.001.1r_KM-443-13C.esp<smiles>CCOC(=O)c1cc(C(=O)[C@@H](OCc2ccccc2)[C@@H](C)O)oc1C</smiles>

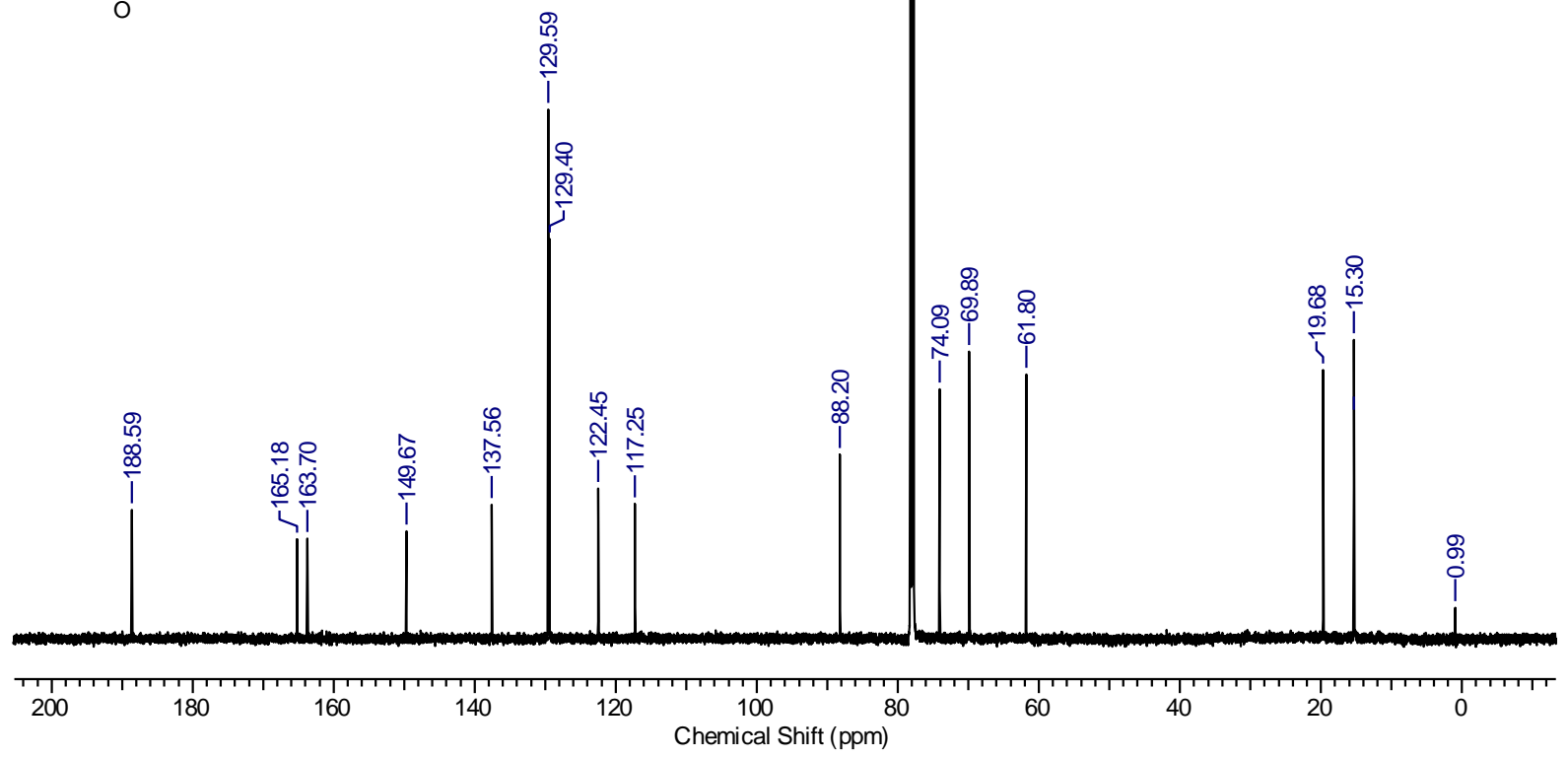




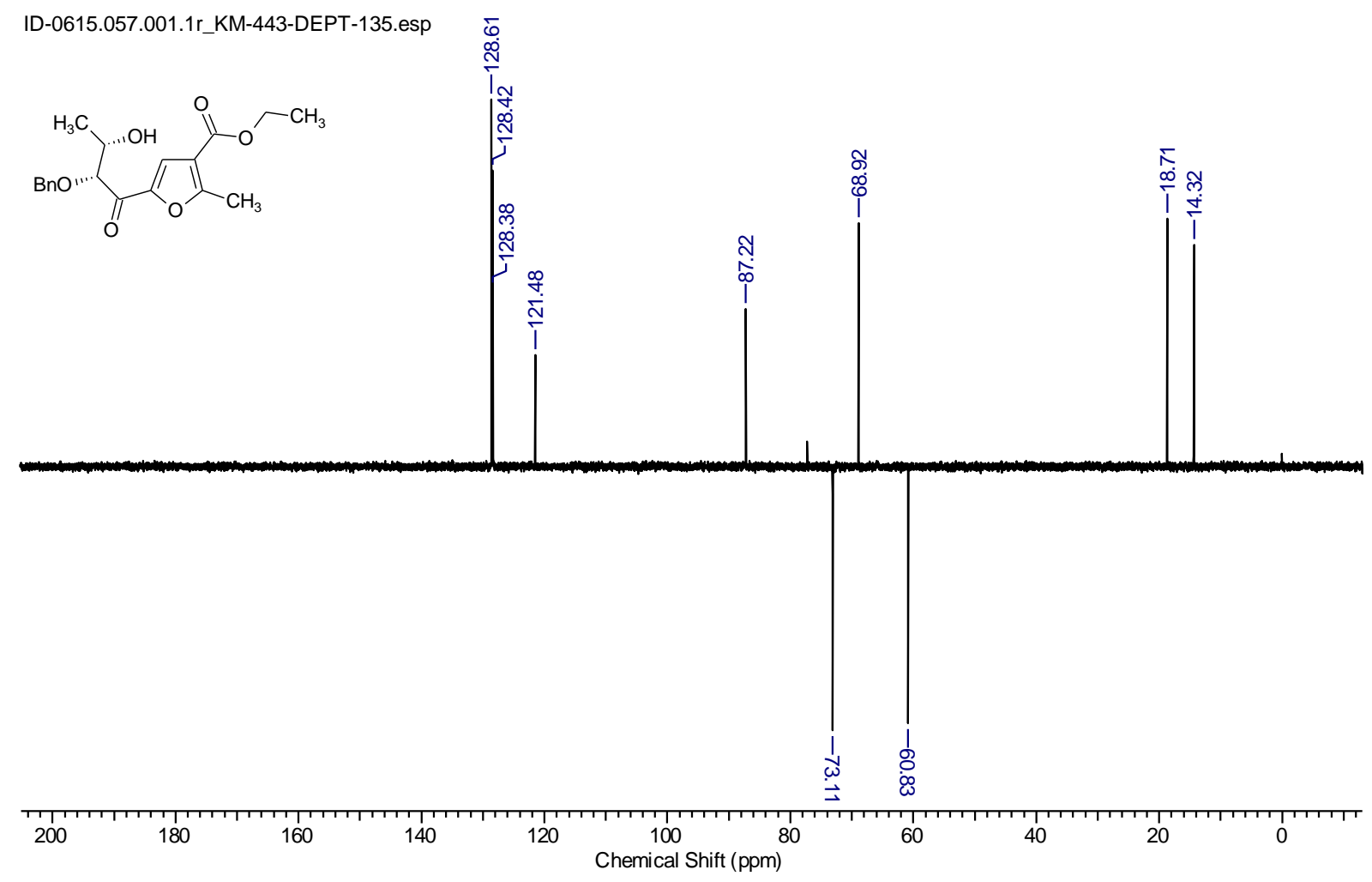

2D (COSY, HMBC, HSQC, and NOESY) NMR spectra of 3a:

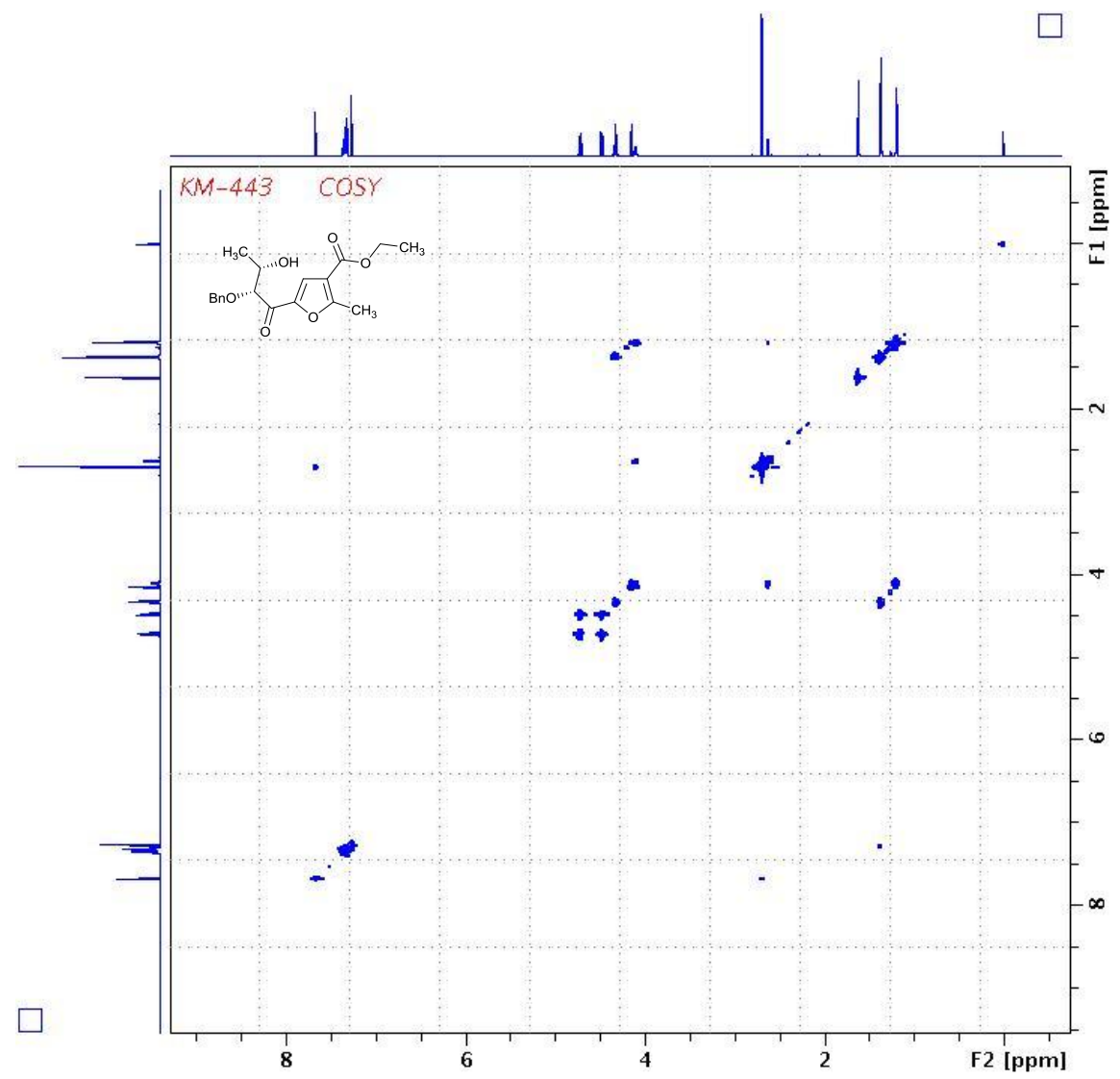




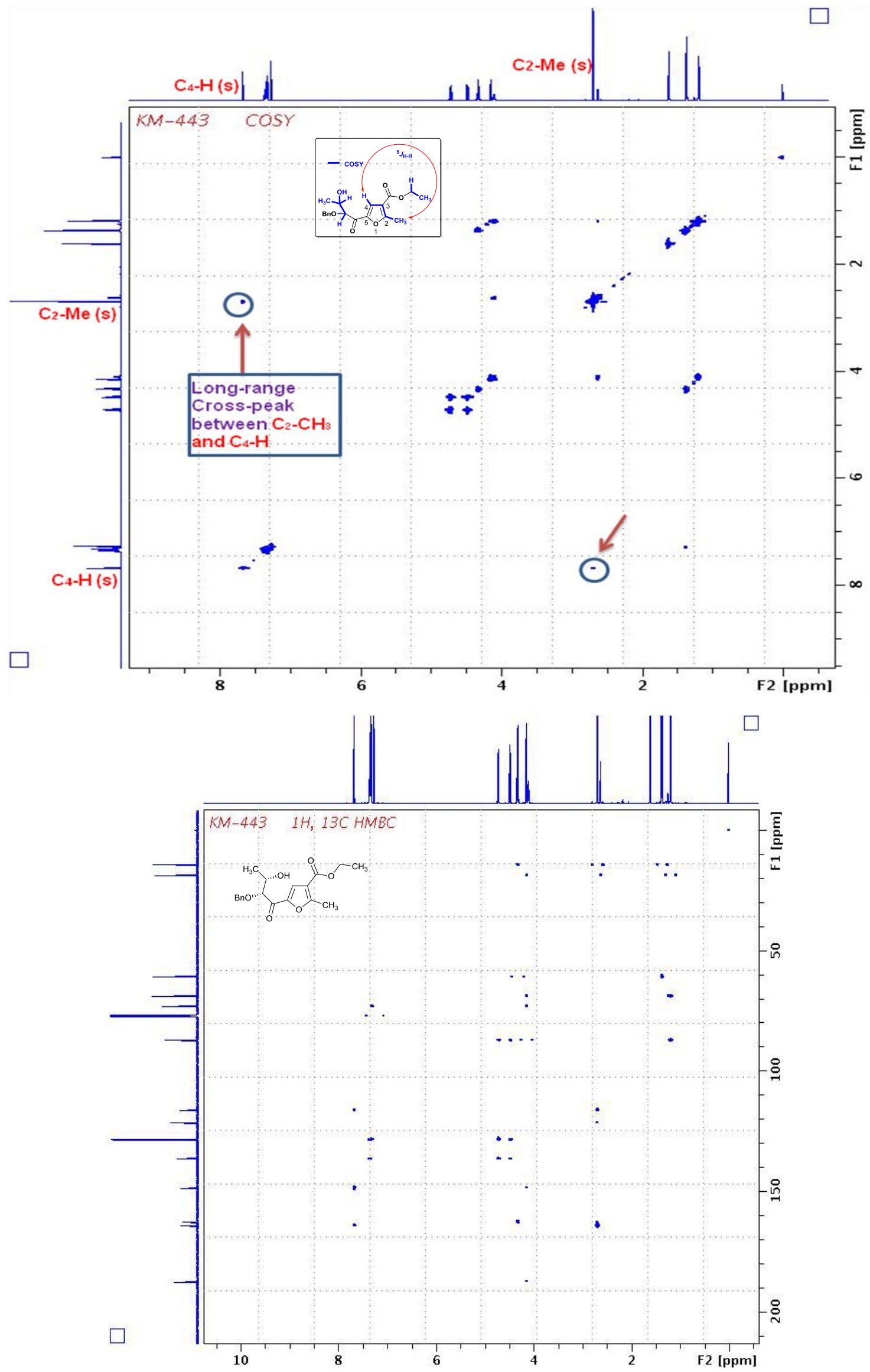



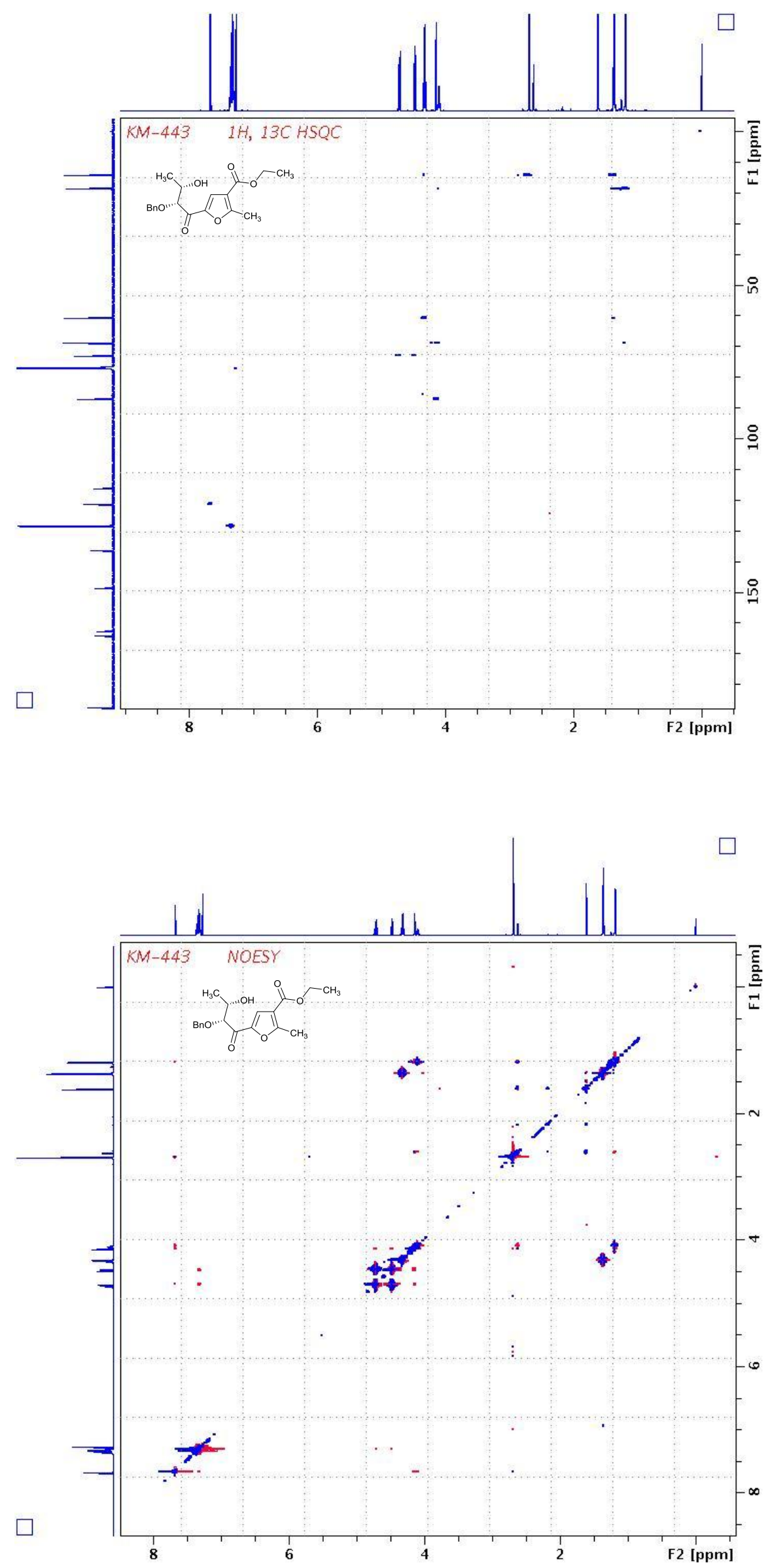


\section{${ }^{1} \mathrm{H},{ }^{13} \mathrm{C}$, DEPT-135 NMR spectra of $3 \mathrm{~b}$ :}

KM-455-1H.001.001.1r.esp_1H.esp

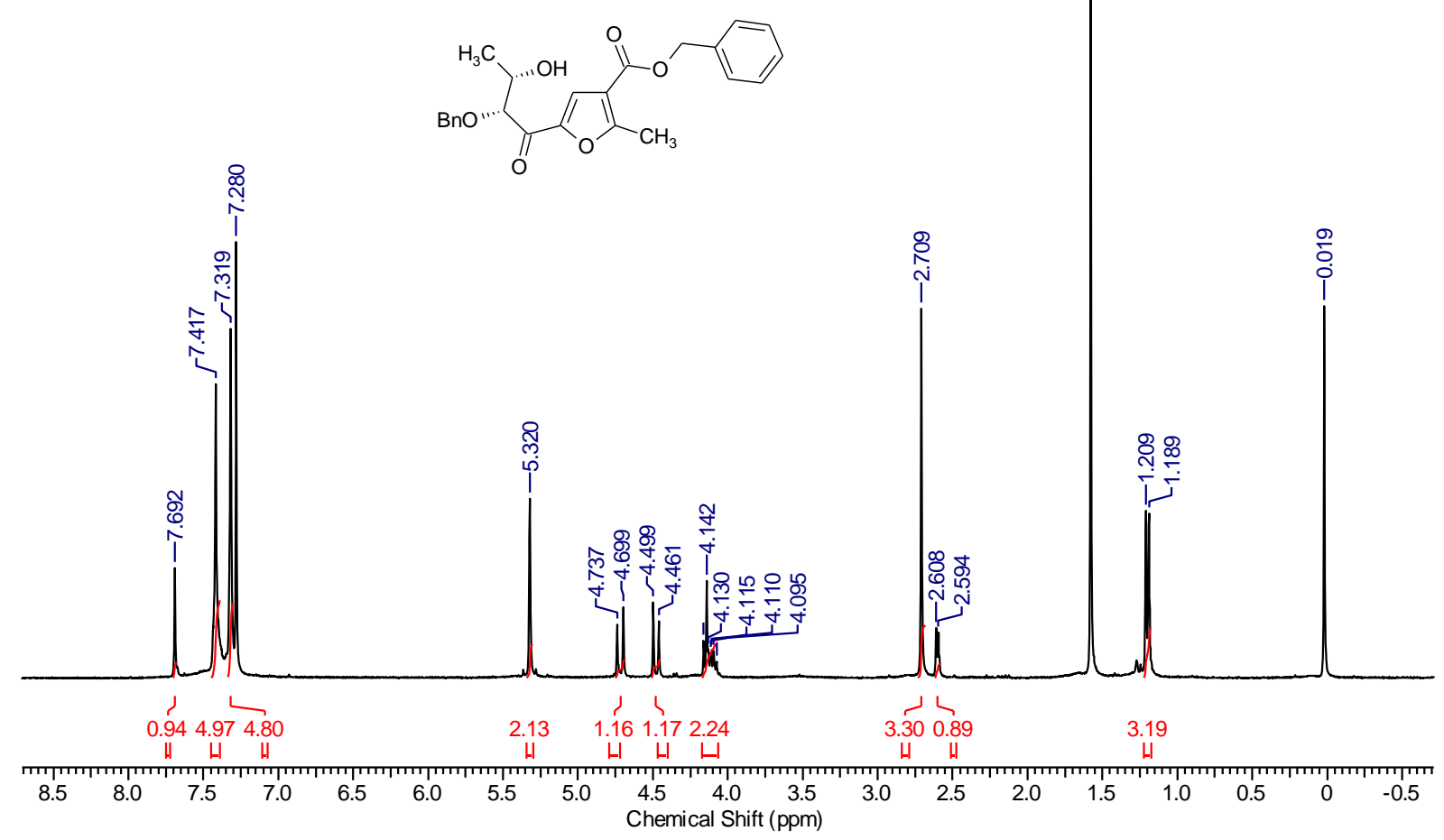

KM-455-C.001.001.1r.esp_13C.esp<smiles>Cc1oc(C(=O)[C@@H](OCc2ccccc2)[C@@H](C)O)cc1C(=O)OCc1ccccc1</smiles>

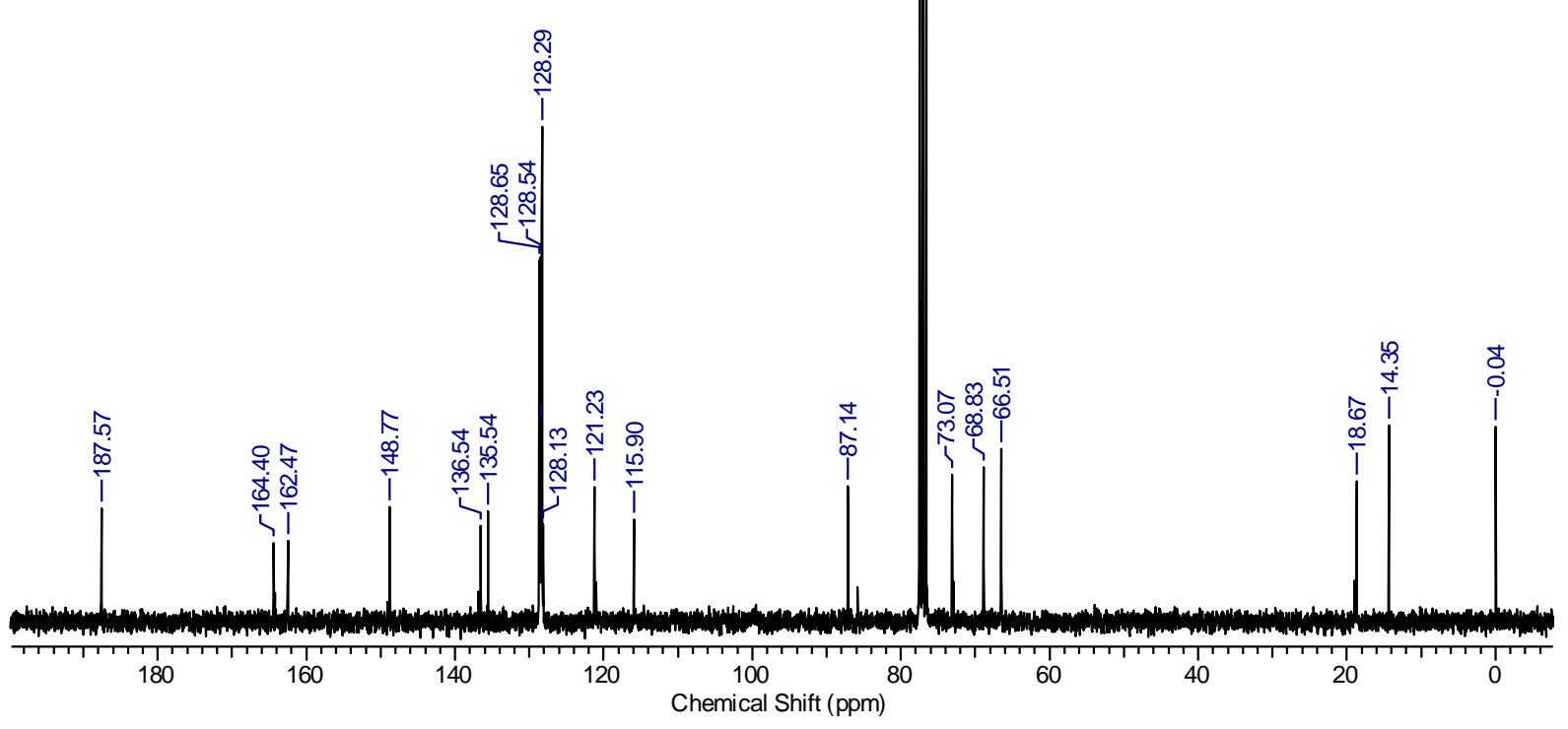




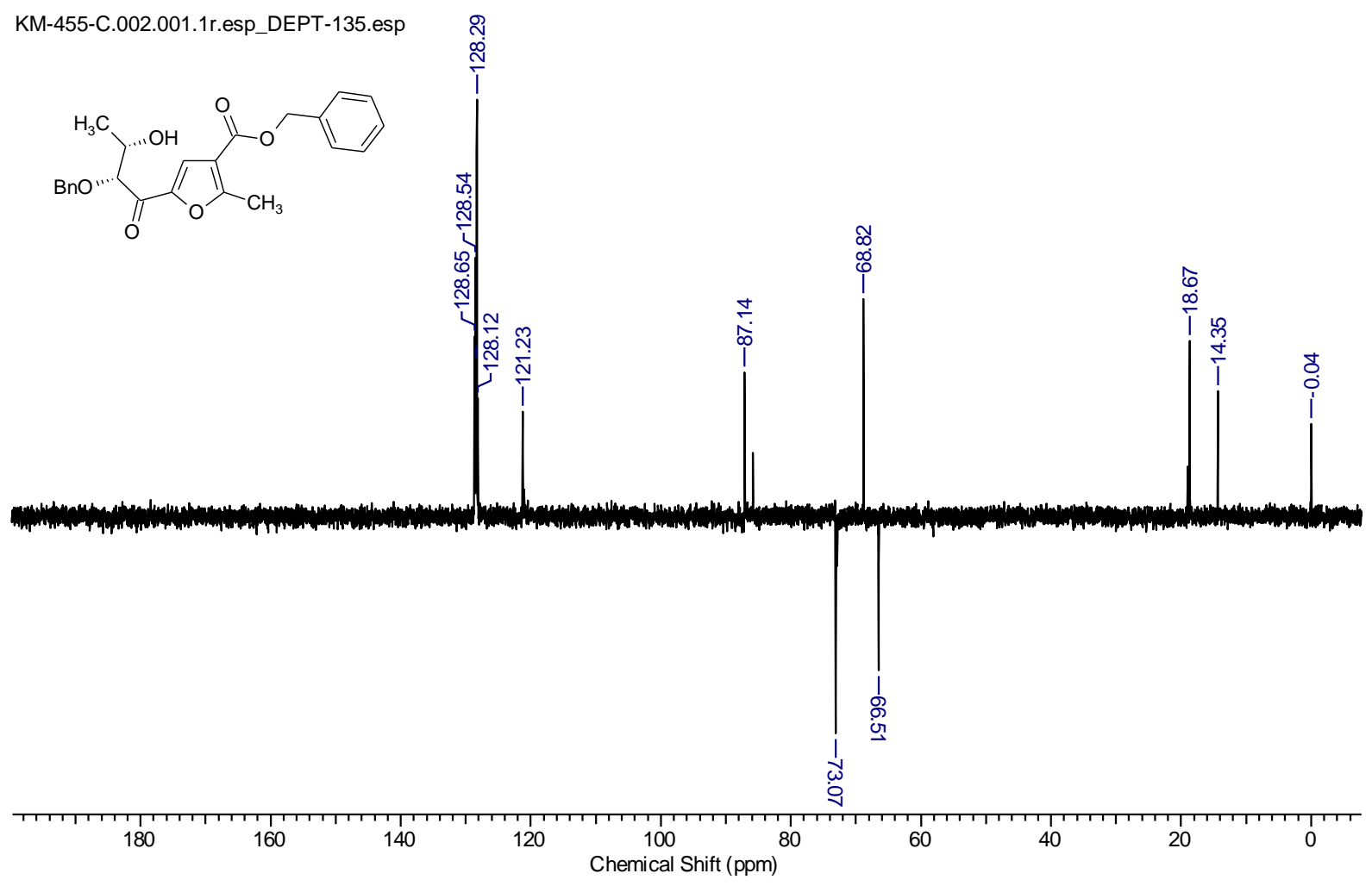

${ }^{1} \mathrm{H},{ }^{13} \mathrm{C}$, DEPT-135 NMR spectra of 3c:

ID-0815.037.001.1r_KM-456-1H.esp

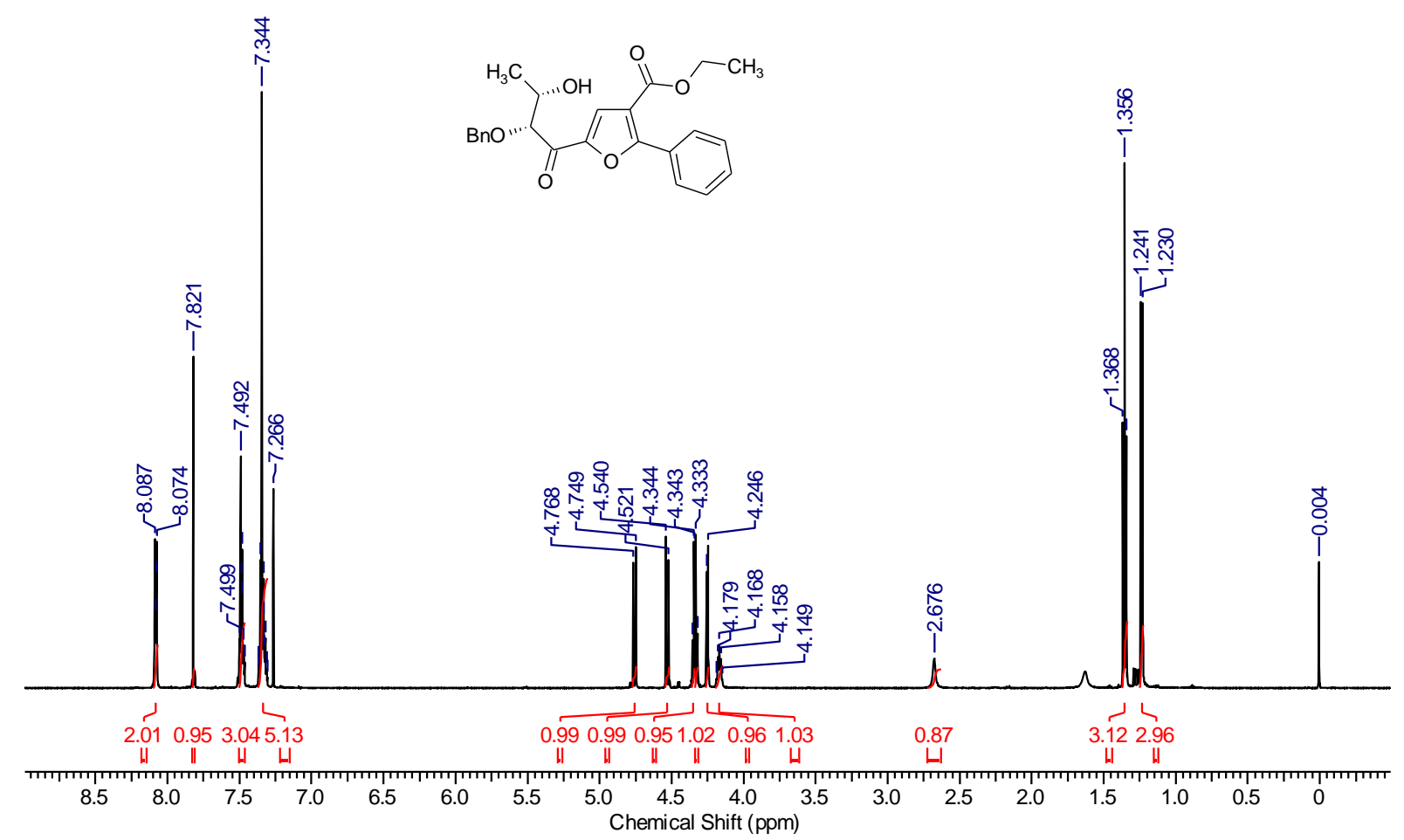



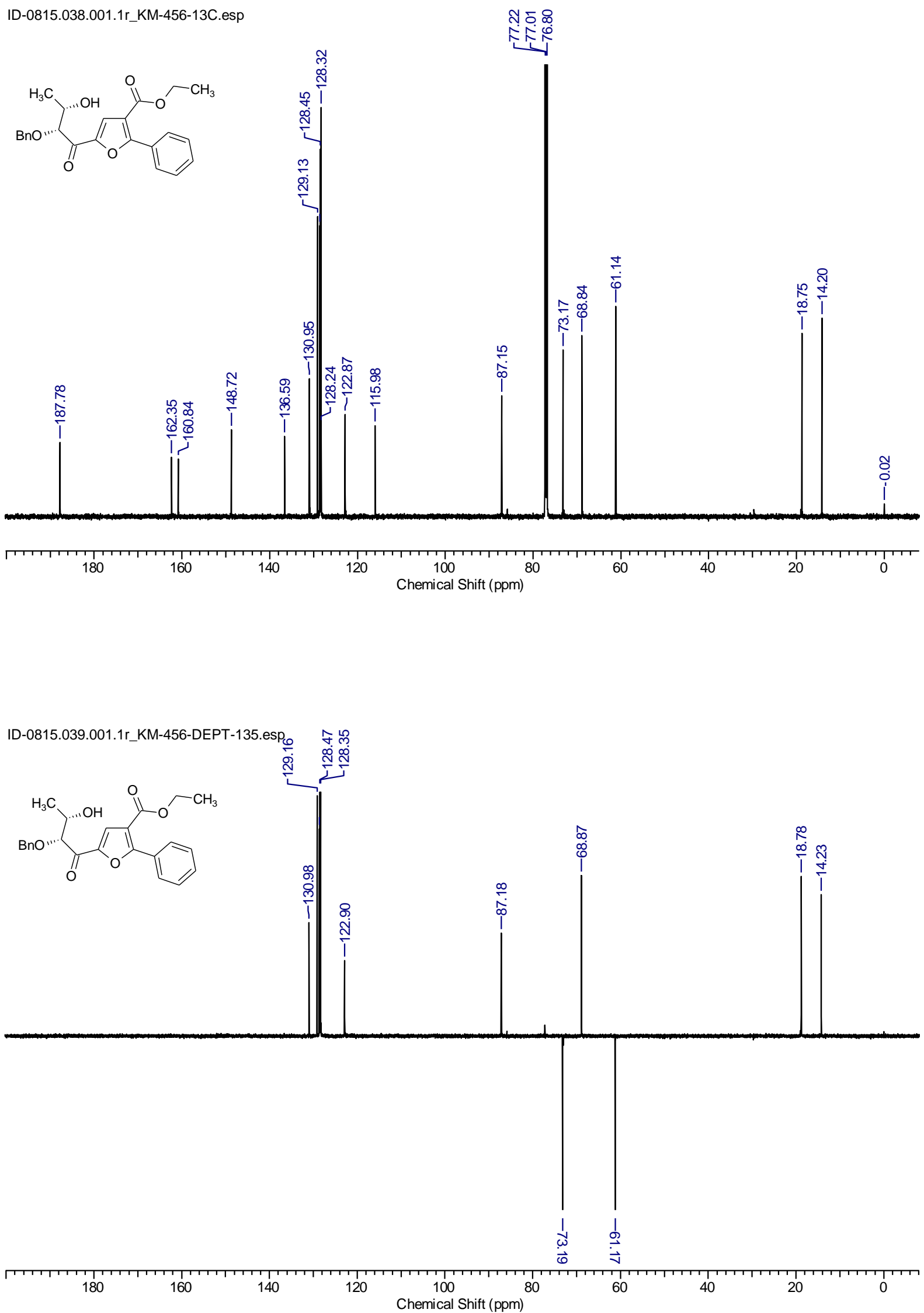
2D (COSY, HMBC, HSQC, and NOESY) NMR spectra of 3c:
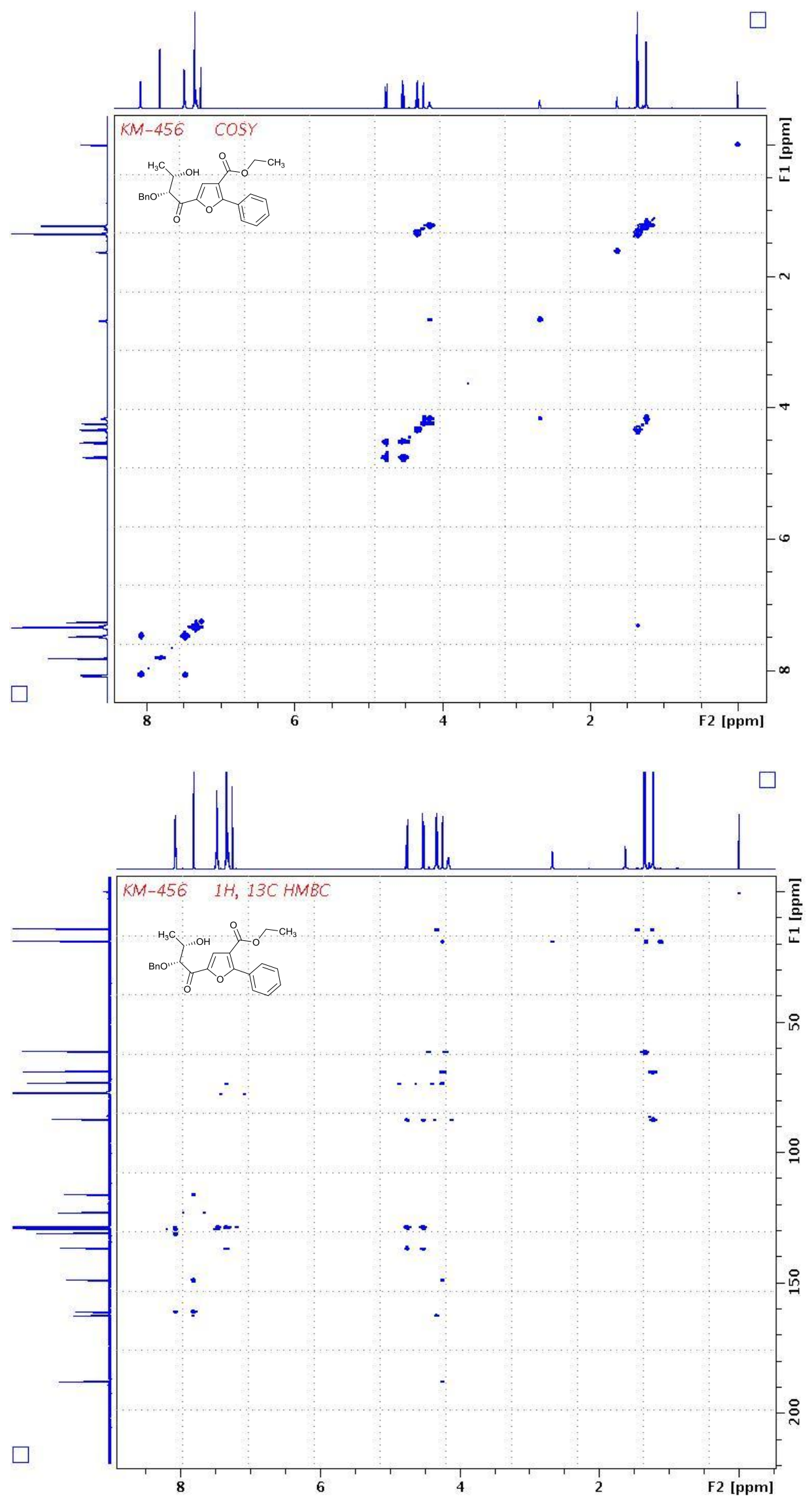

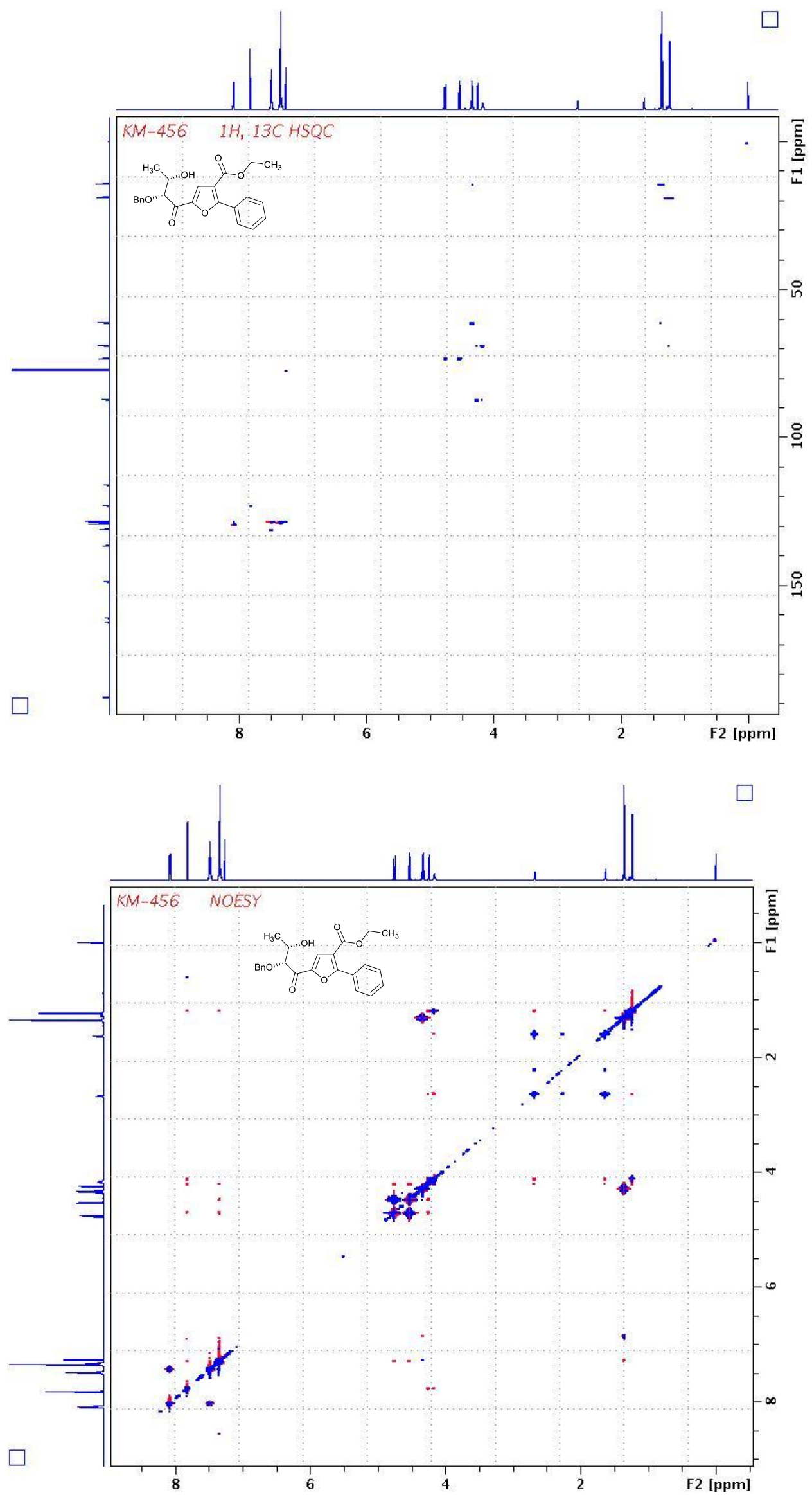

S69 


\section{${ }^{1} \mathrm{H},{ }^{13} \mathrm{C}$, DEPT-135 NMR spectra of 3d:}

ID-0915.013.001.1r.esp_KM-466-1H.esp

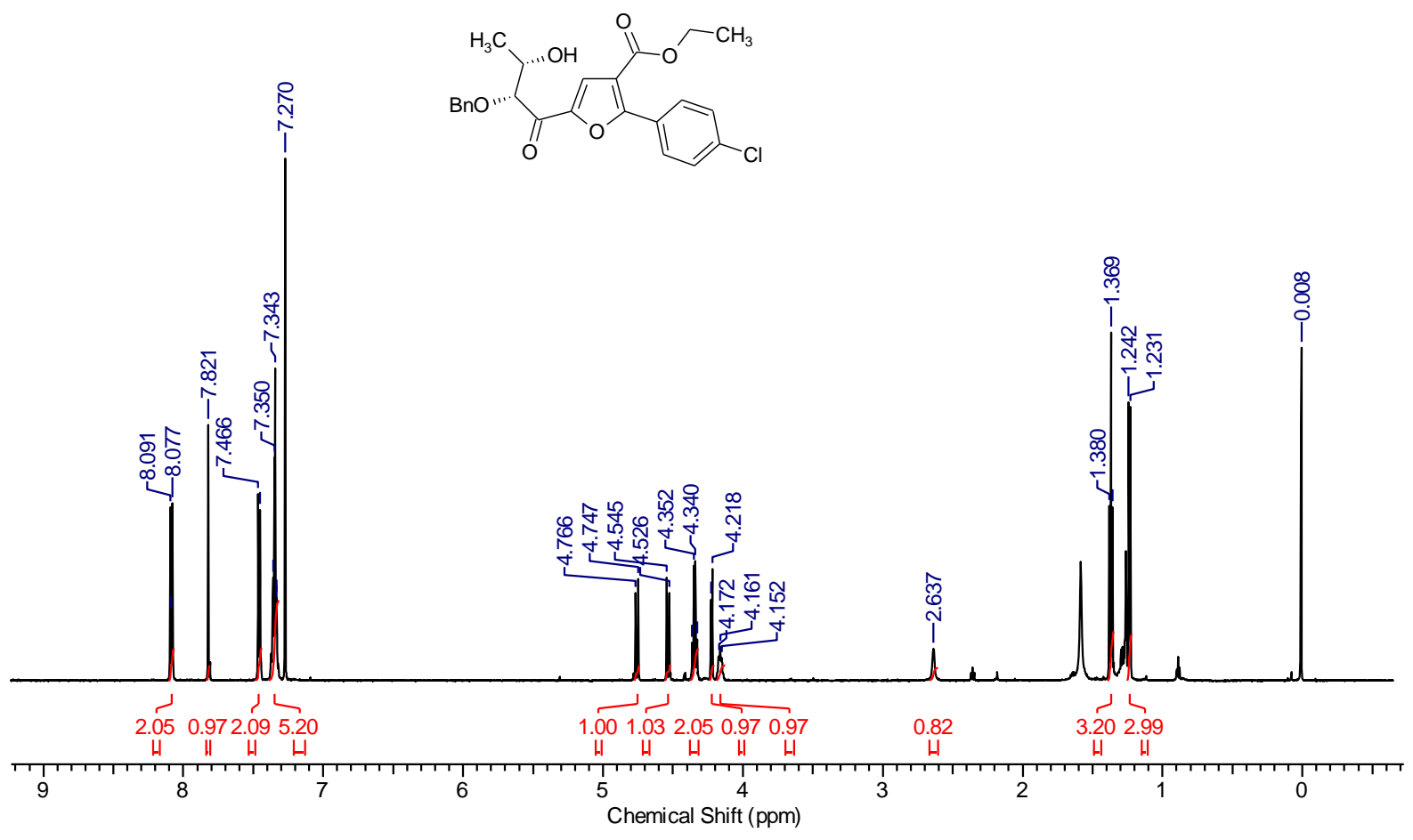

ID-0915.014.001.1r.esp_KM-466-13C.esp

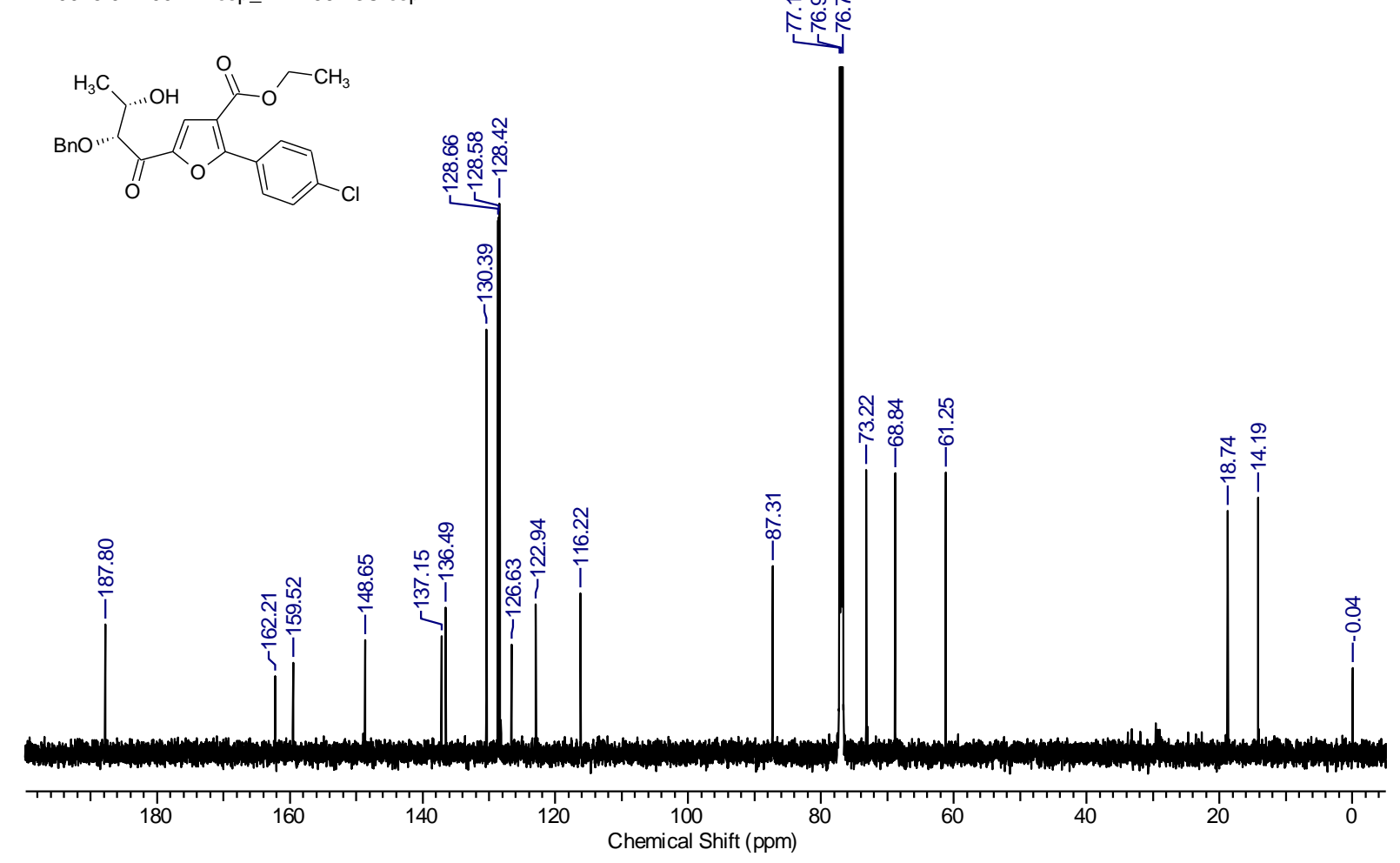




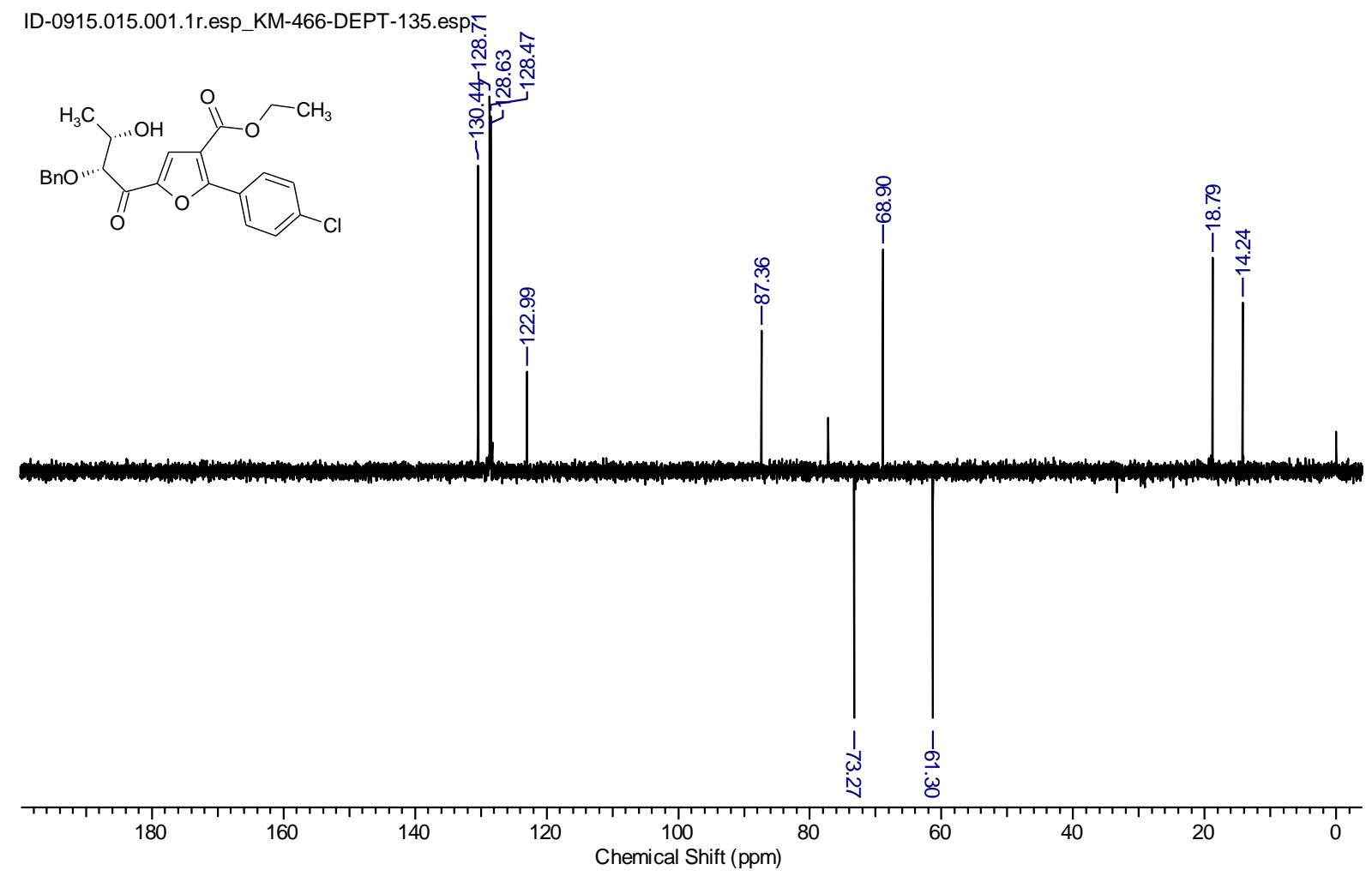

${ }^{1} \mathrm{H},{ }^{13} \mathrm{C}$, DEPT-135 NMR spectra of 3e:

ID-0915.005.001.1r.esp_KM-485-1H.esp<smiles>COC(=O)c1cc(C(=O)[C@@H](O)[C@H](Cc2ccccc2)C(O)O)oc1OC</smiles>

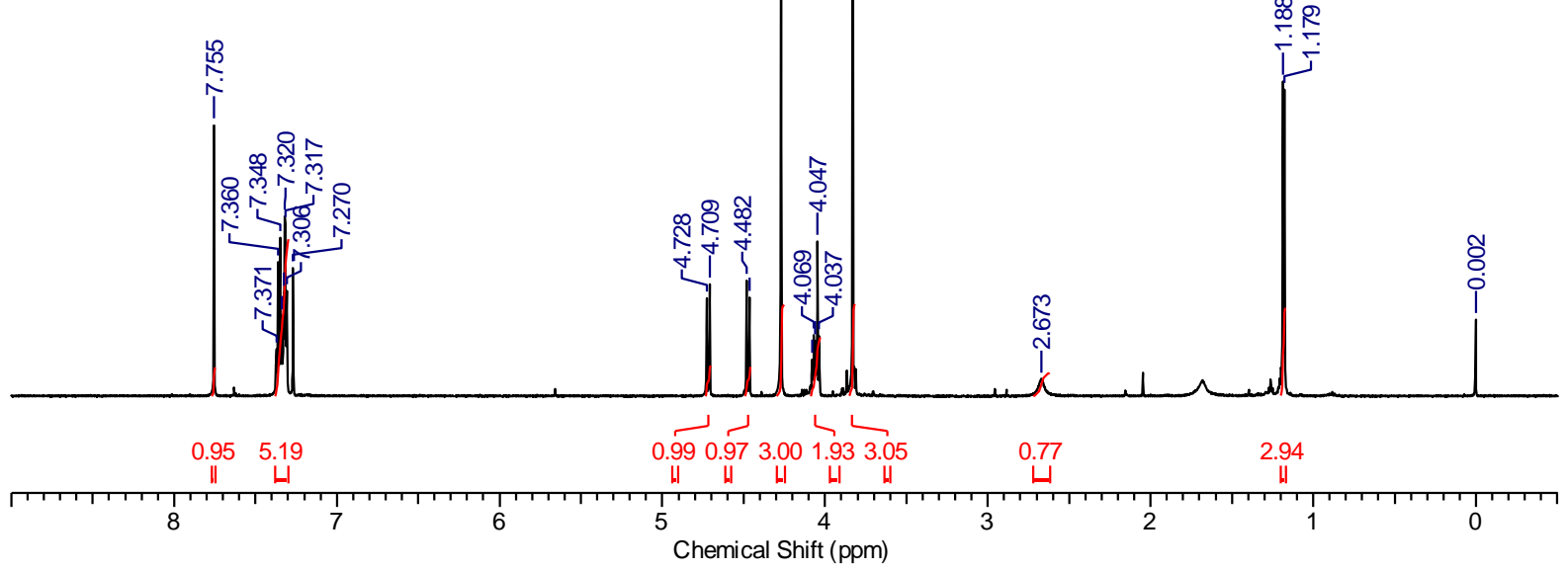



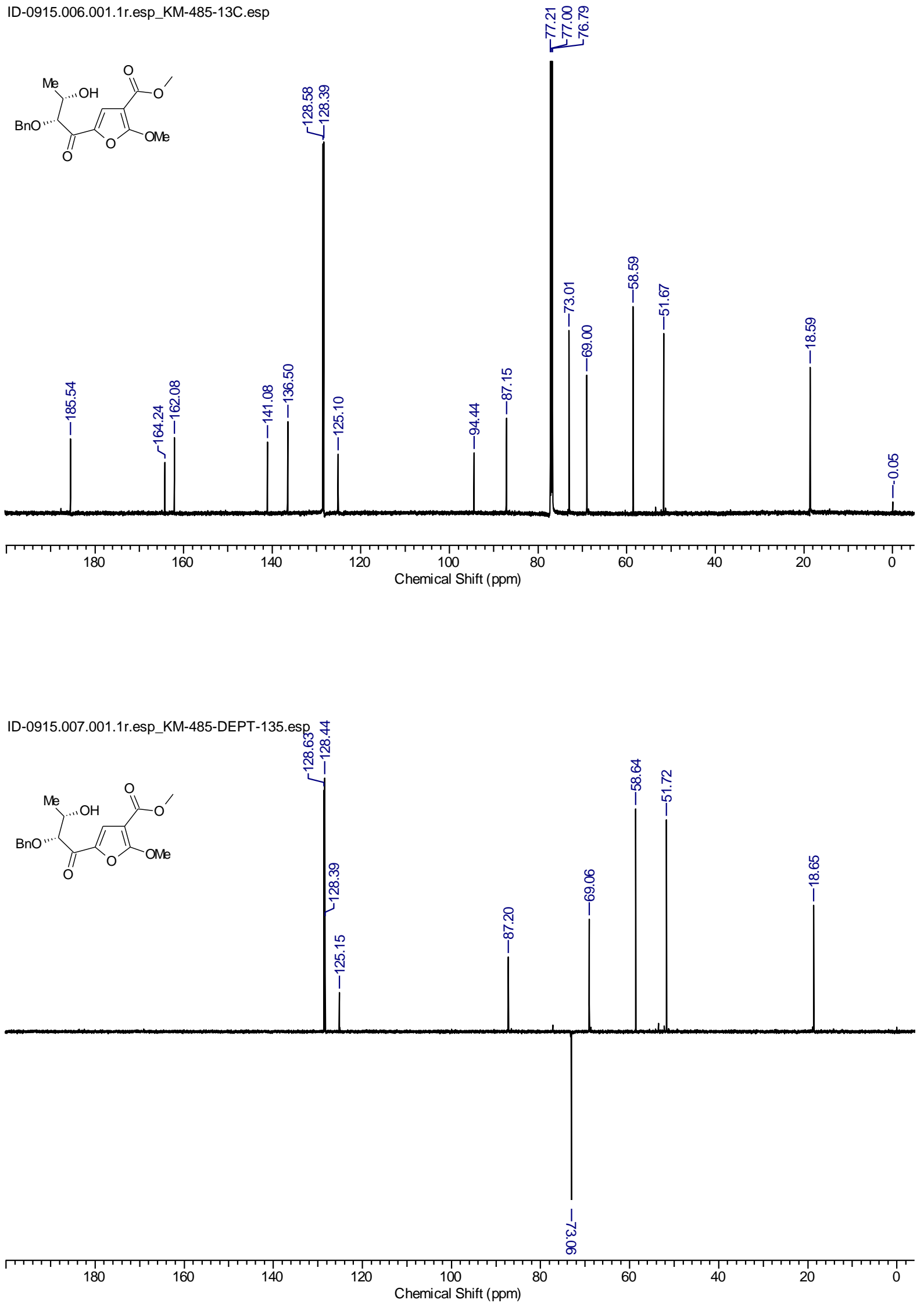


\section{${ }^{1} \mathrm{H},{ }^{13} \mathrm{C}$, DEPT-135 NMR spectra of $3 f$ :}

ID-0815.121.001.1r_KM-483-1H.esp<smiles>CCOC(=O)c1cc(C(=O)[C@@H](O)[C@H](C)O)oc1[N+](=O)[O-]</smiles>

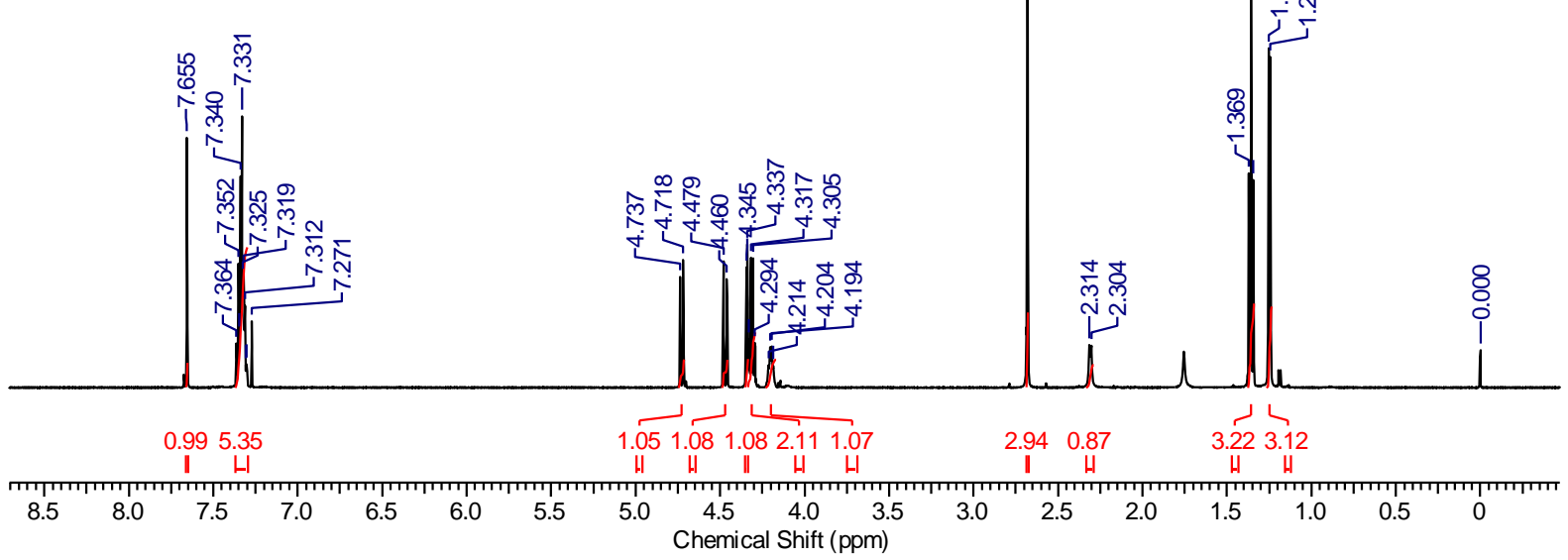

ID-0815.122.001.1r_KM-483-13C.esp<smiles>CCOC(=O)c1cc(C(=O)[C@@H](O)[C@H](C)O)oc1[N+](=O)[O-]</smiles>

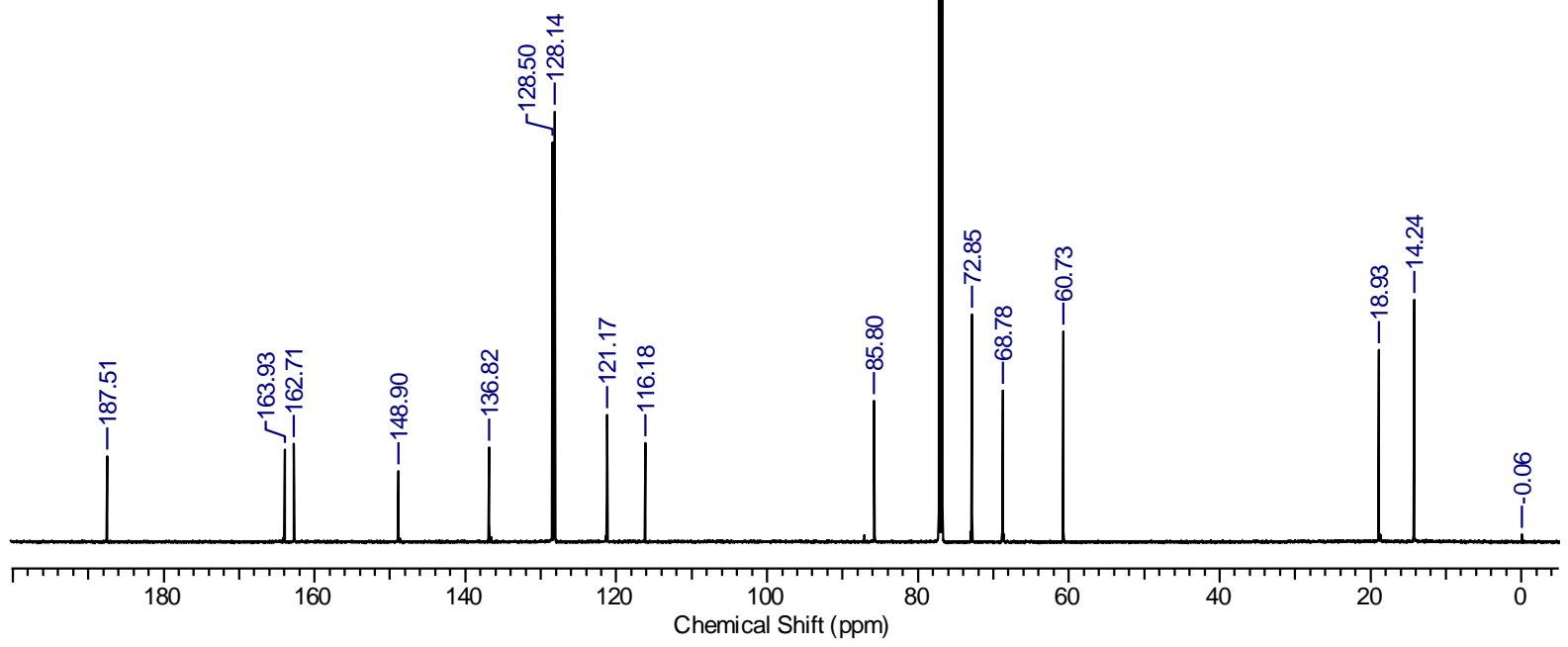




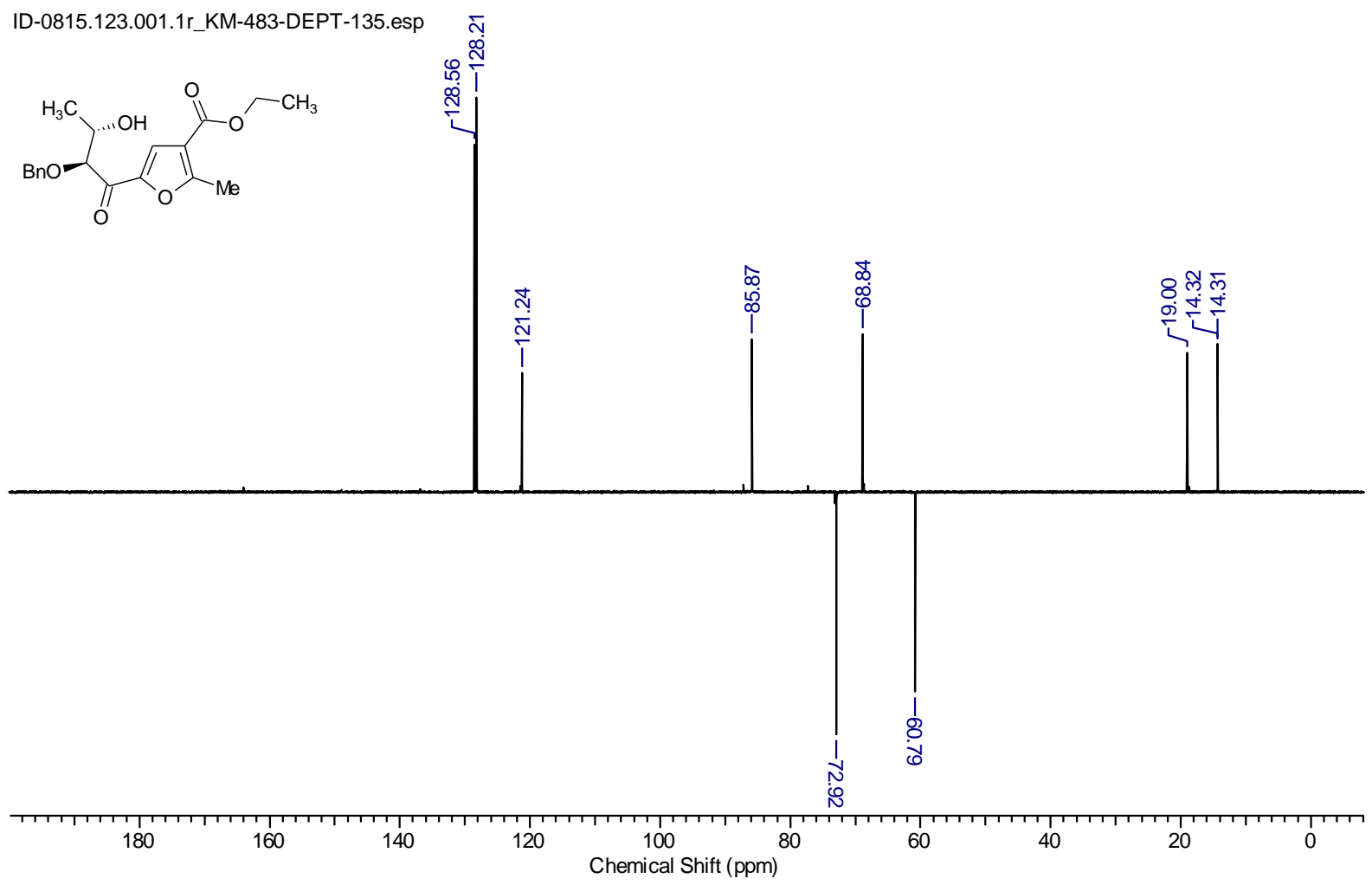

2D (COSY, HMBC, HSQC, and NOESY) NMR spectra of 3f:

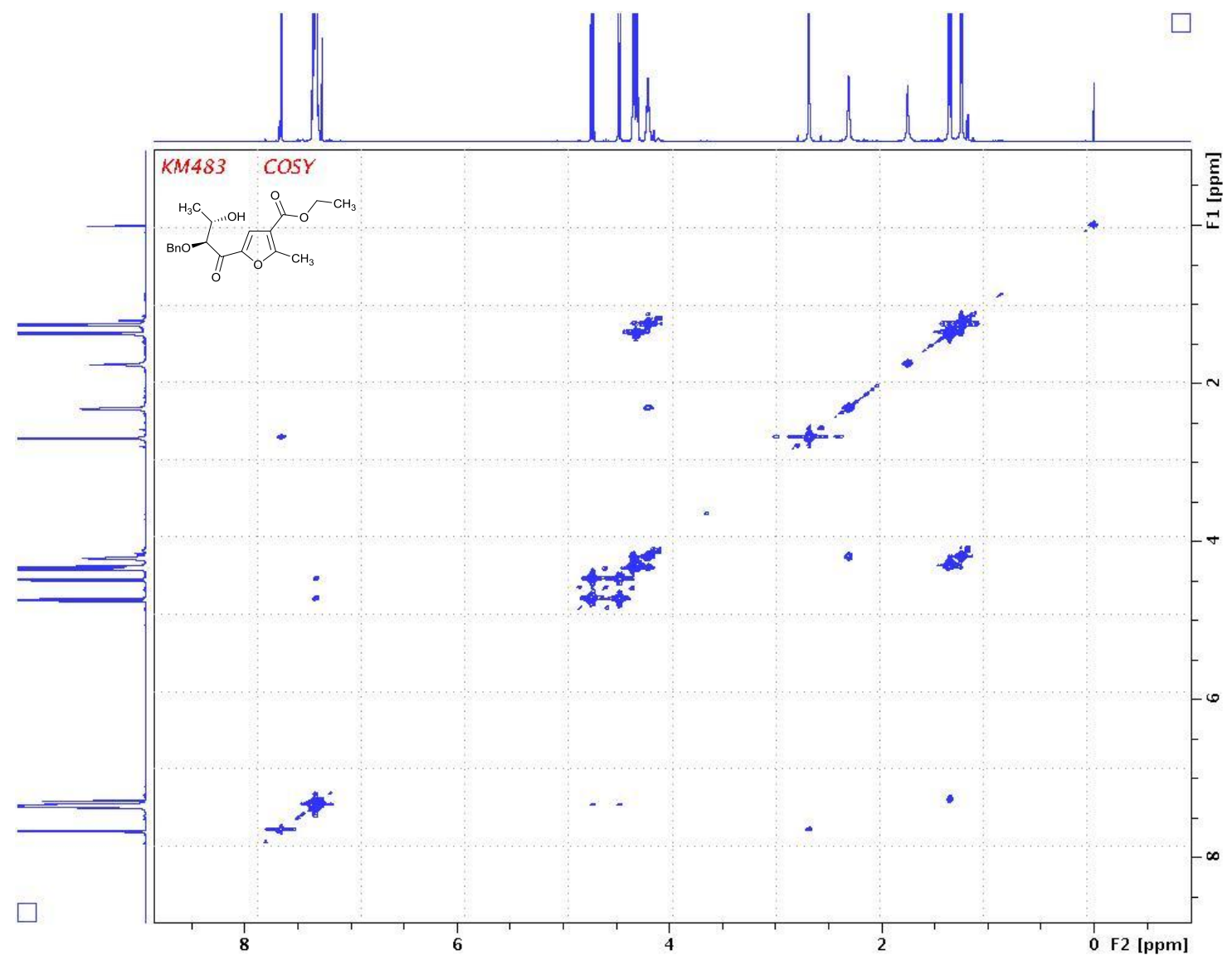



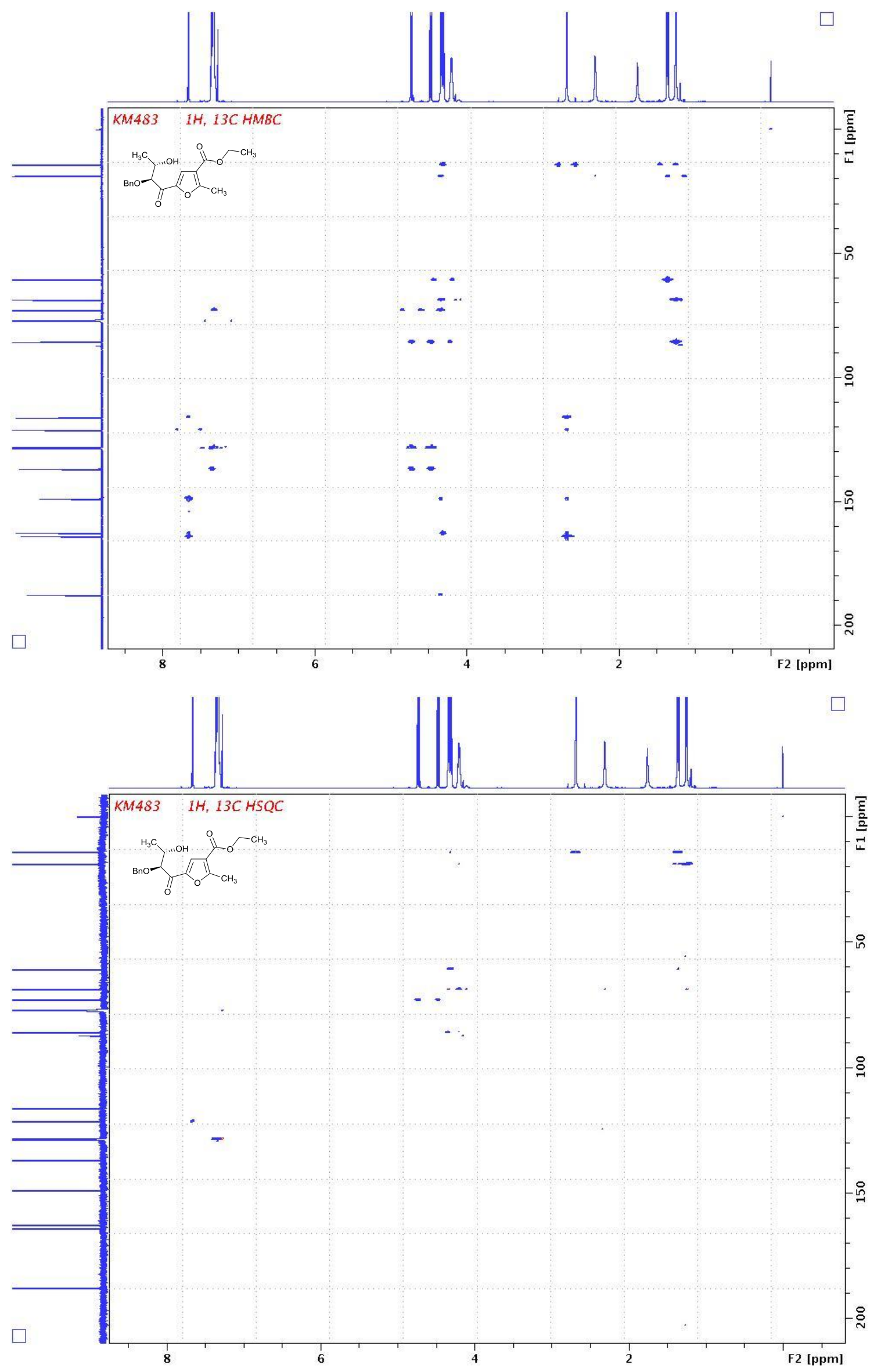


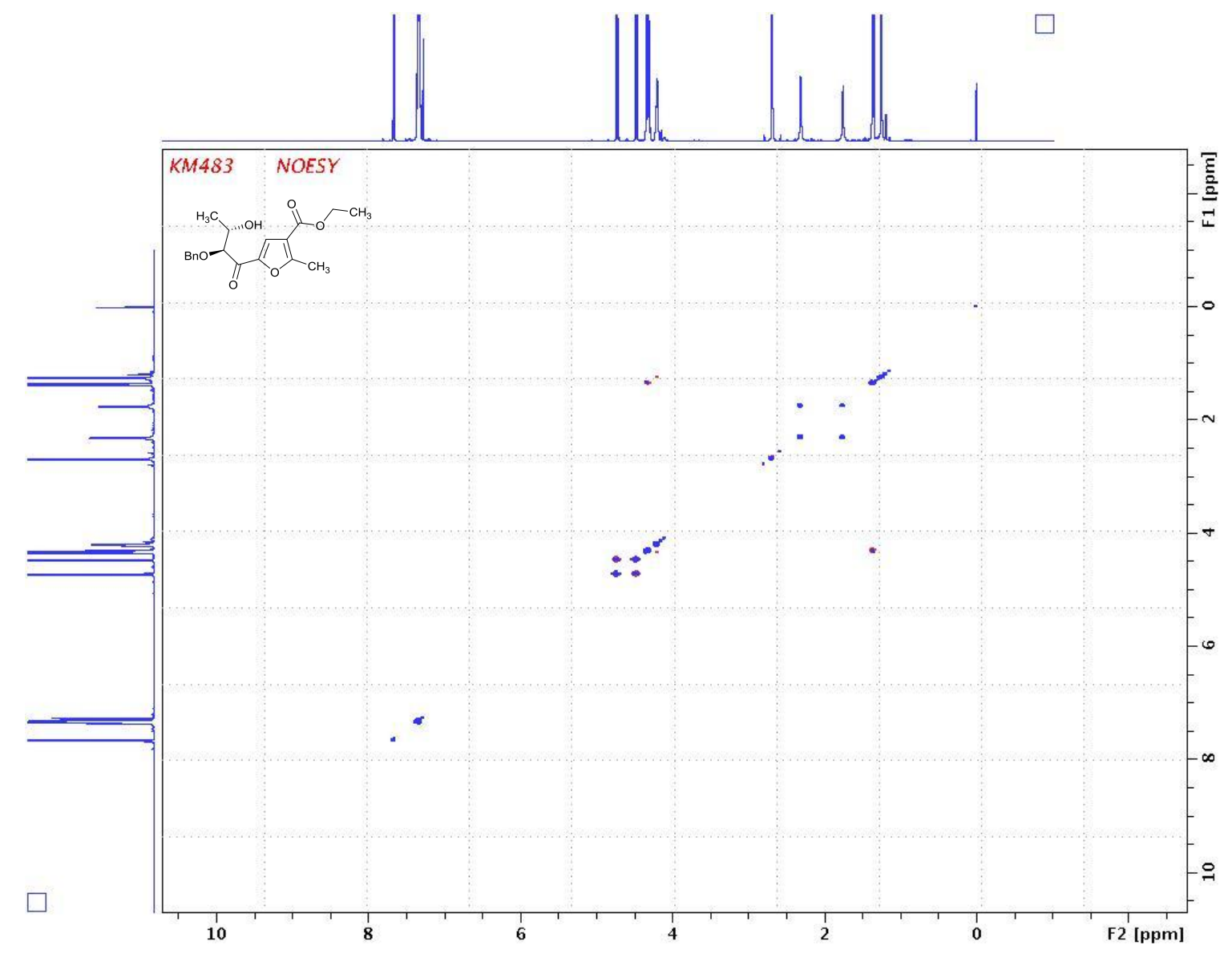

${ }^{1} \mathrm{H},{ }^{13} \mathrm{C}$, DEPT-135 NMR spectra of 3g:

KM-469-1H.001.001.1r.esp_1H.esp

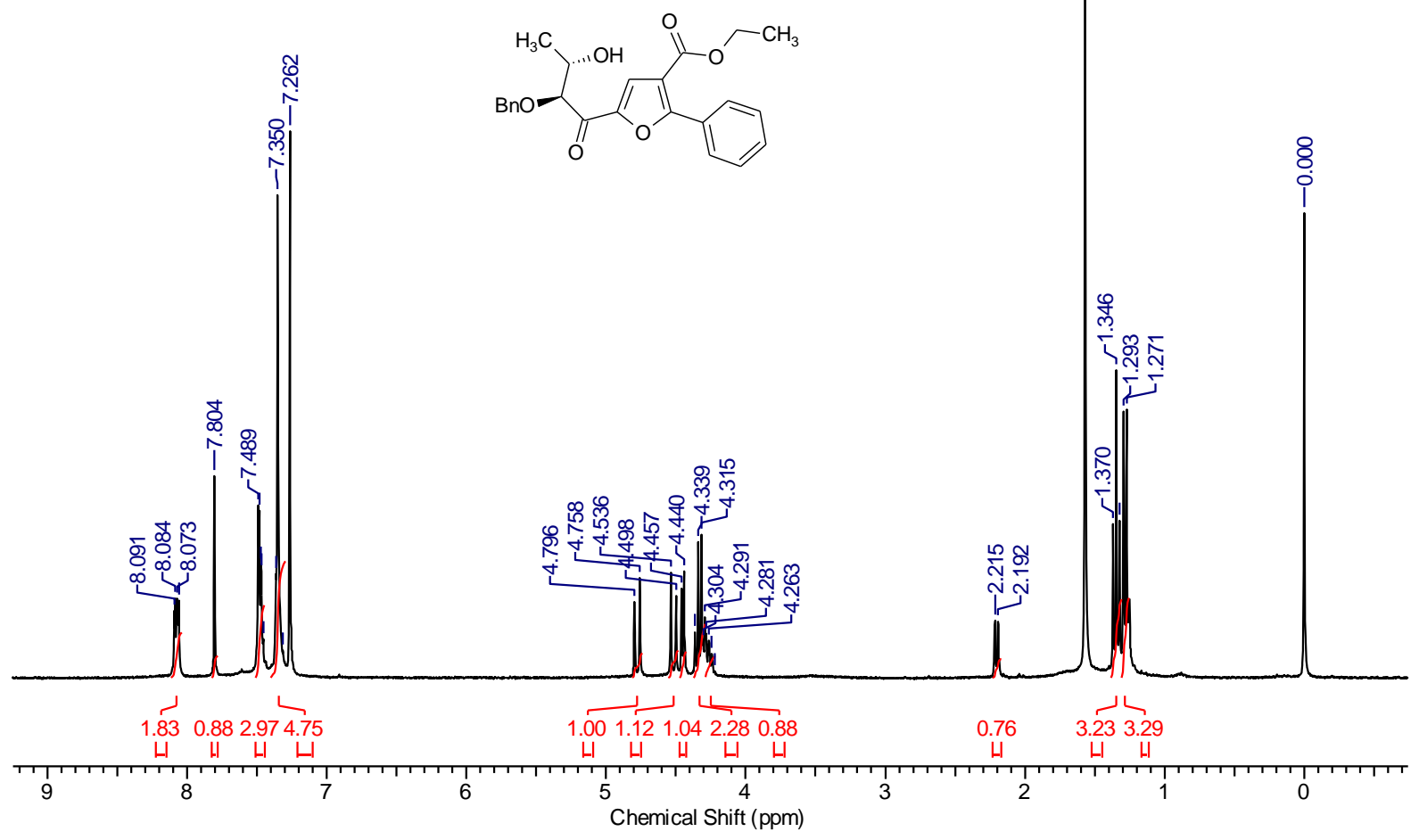



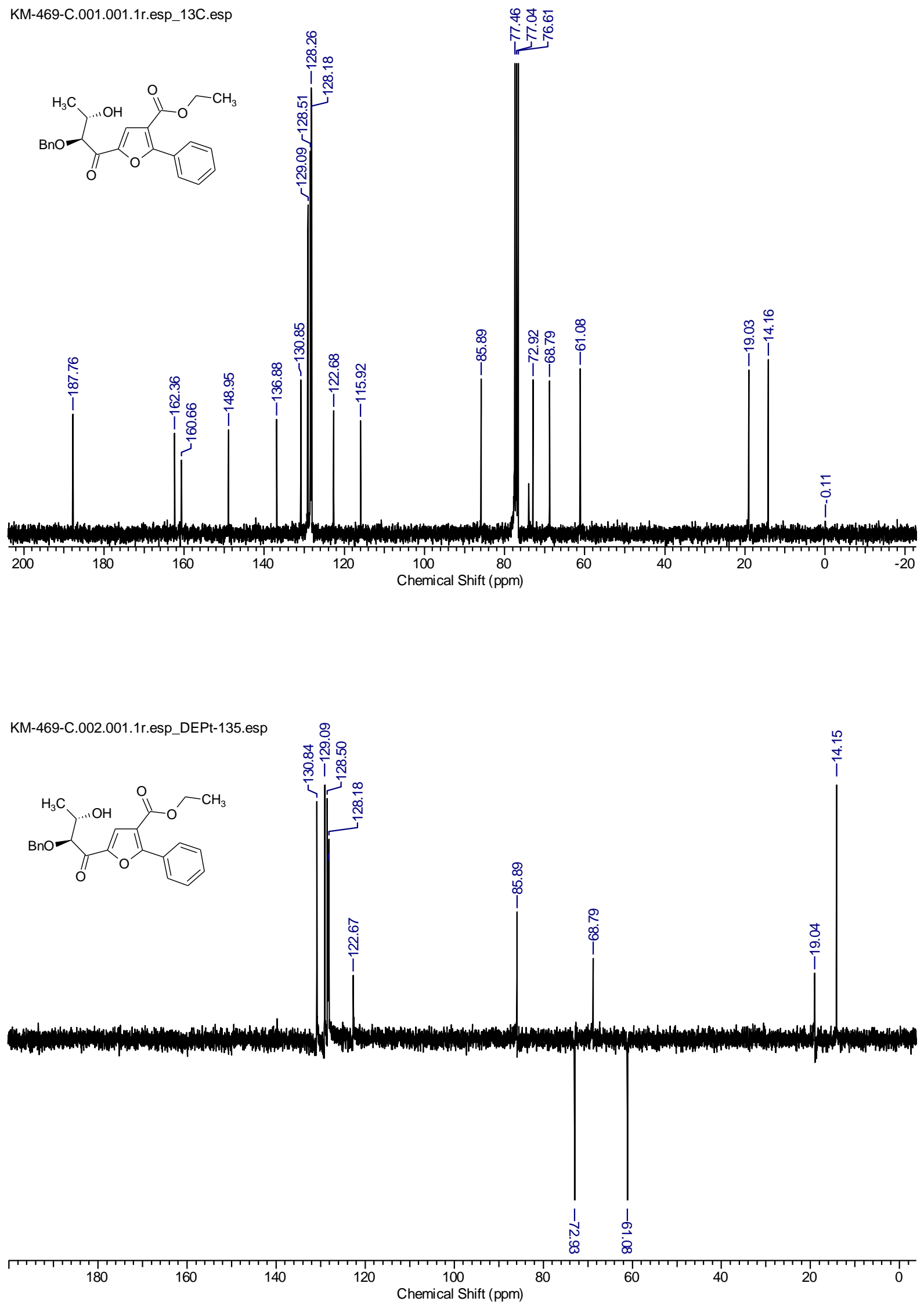


\section{${ }^{1} \mathrm{H},{ }^{13} \mathrm{C}$, DEPT-135 NMR spectra of $3 \mathrm{~h}$ :}

ID-0915.001.001.1R.ESP_KM-484-1H.ESP<smiles>COC(=O)c1cc(C(=O)[C@@H](O)[C@H](C)O)oc1C</smiles>

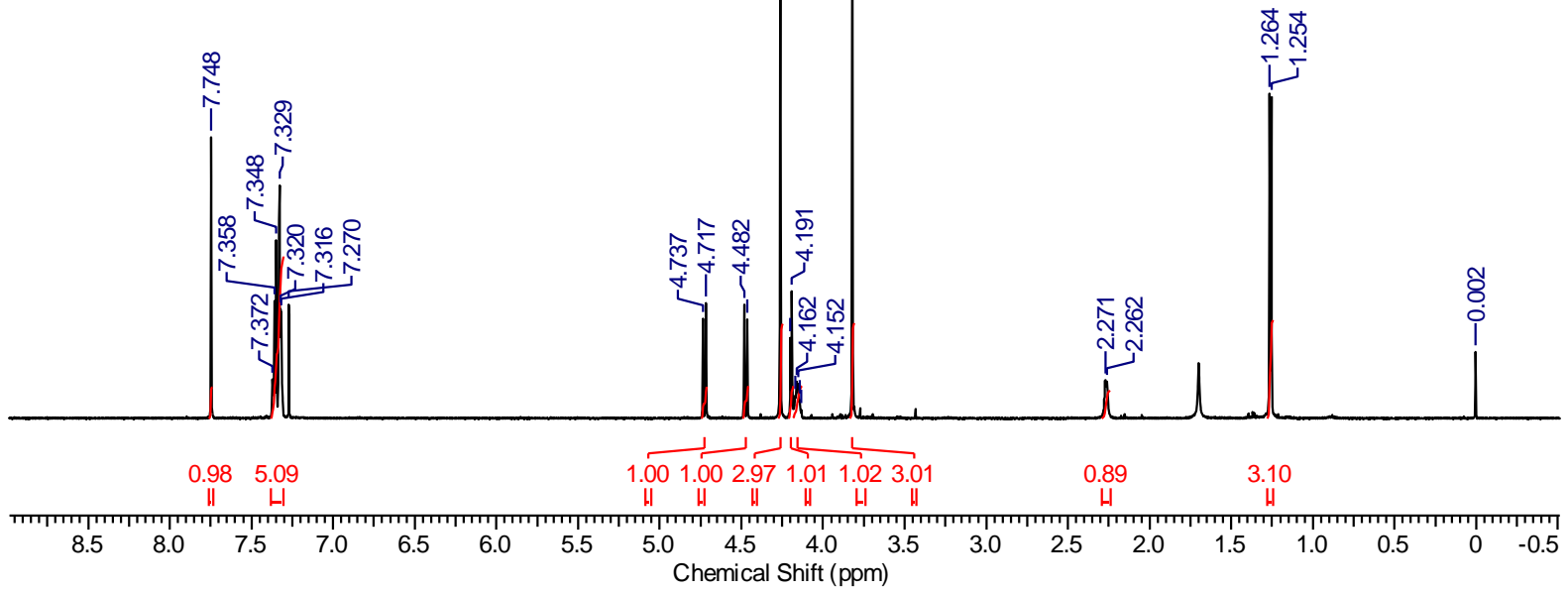

ID-0915.002.001.1R.ESP_KM-484-13C.ESP
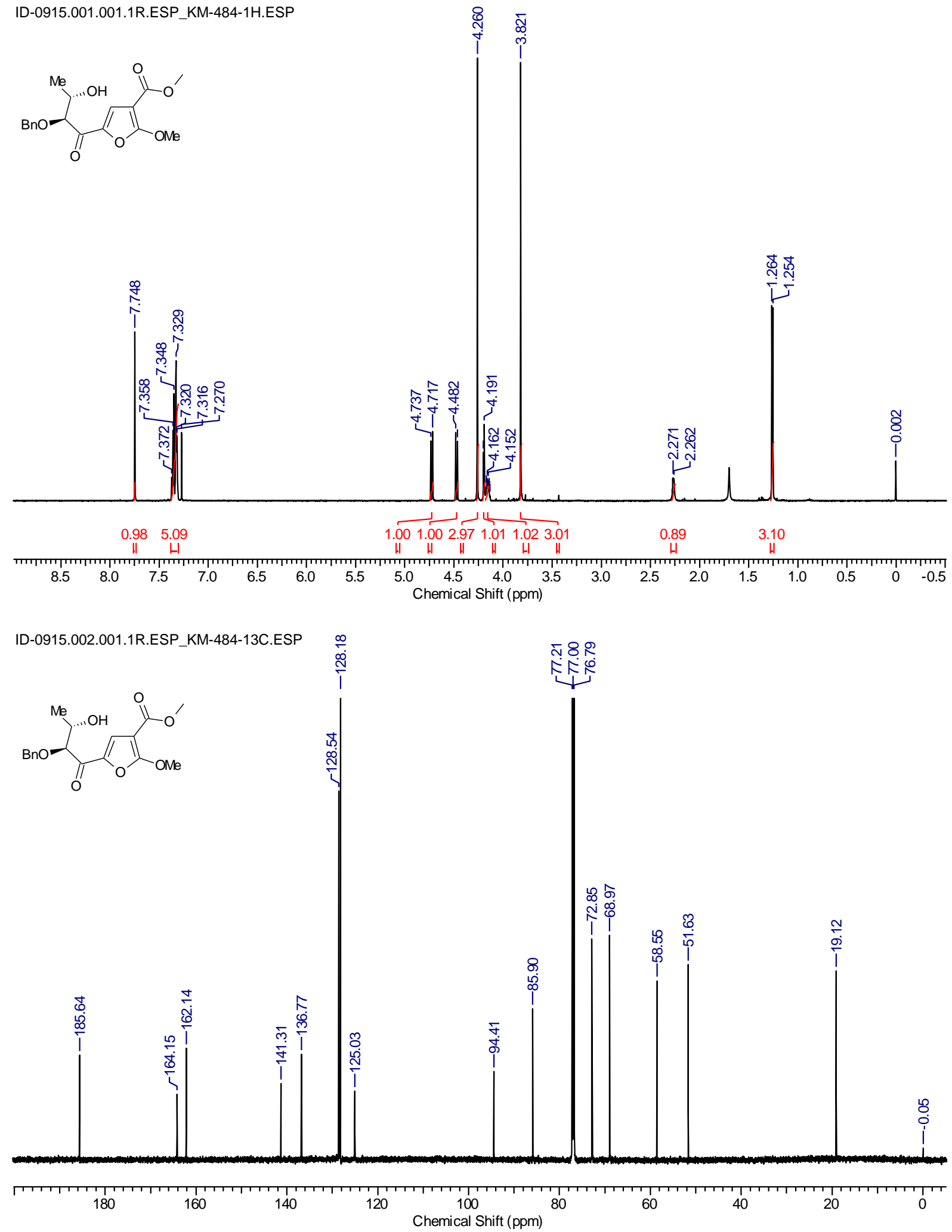


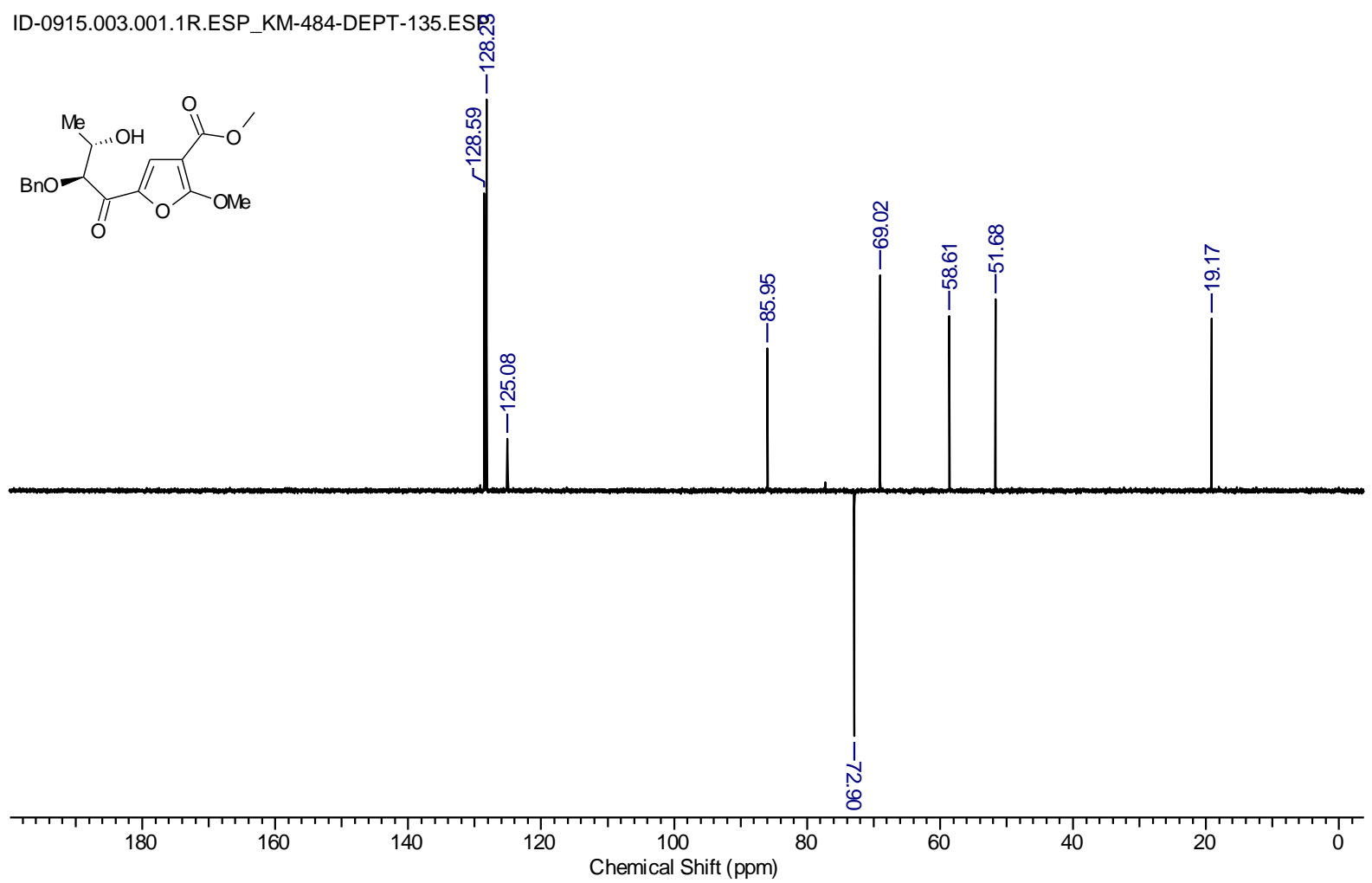

${ }^{1} \mathrm{H},{ }^{13} \mathrm{C}$, DEPT-135 NMR spectra of 3i: 


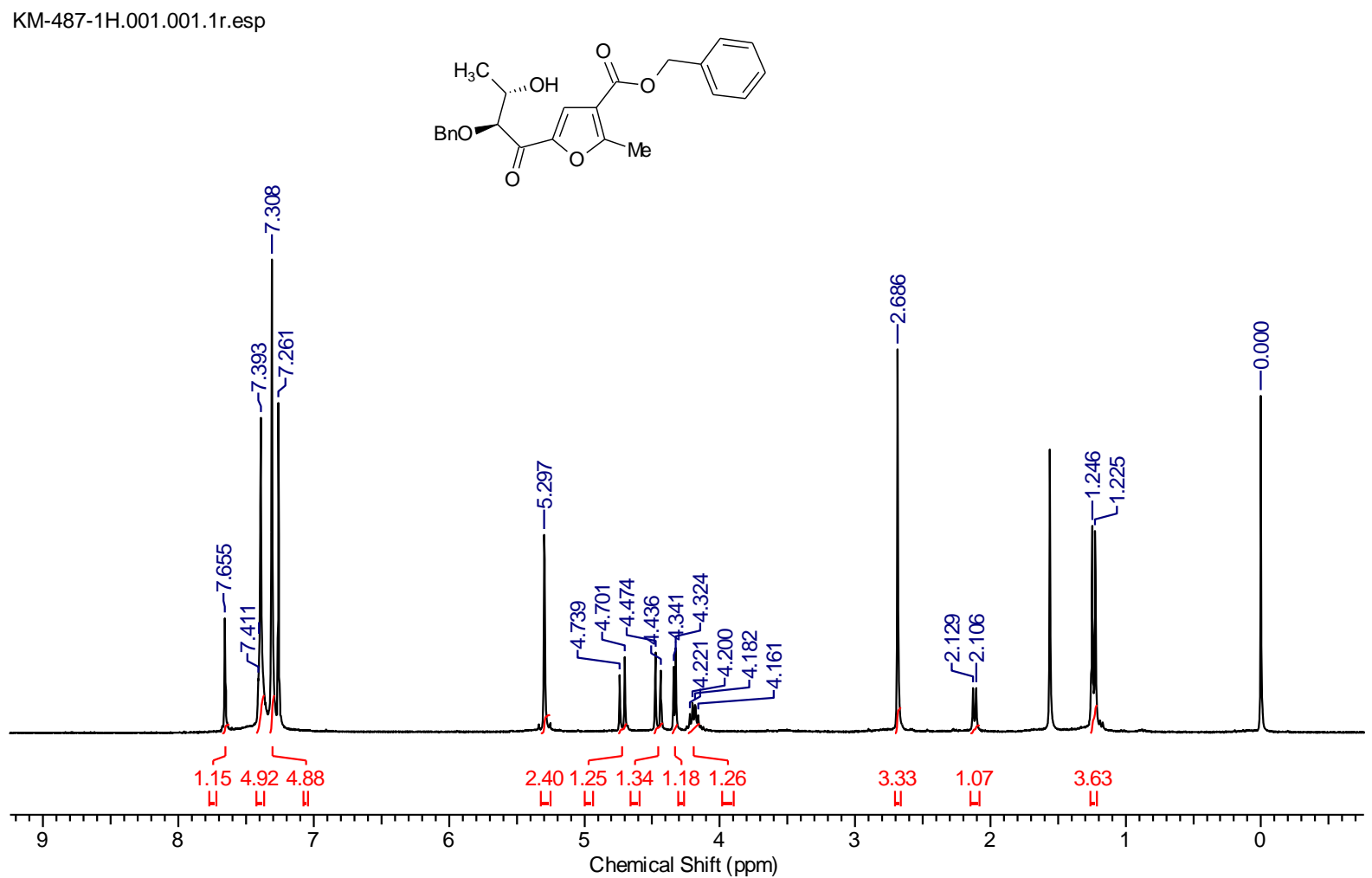

KM-487-C.001.001.1r.esp_KM-487-13C.esp

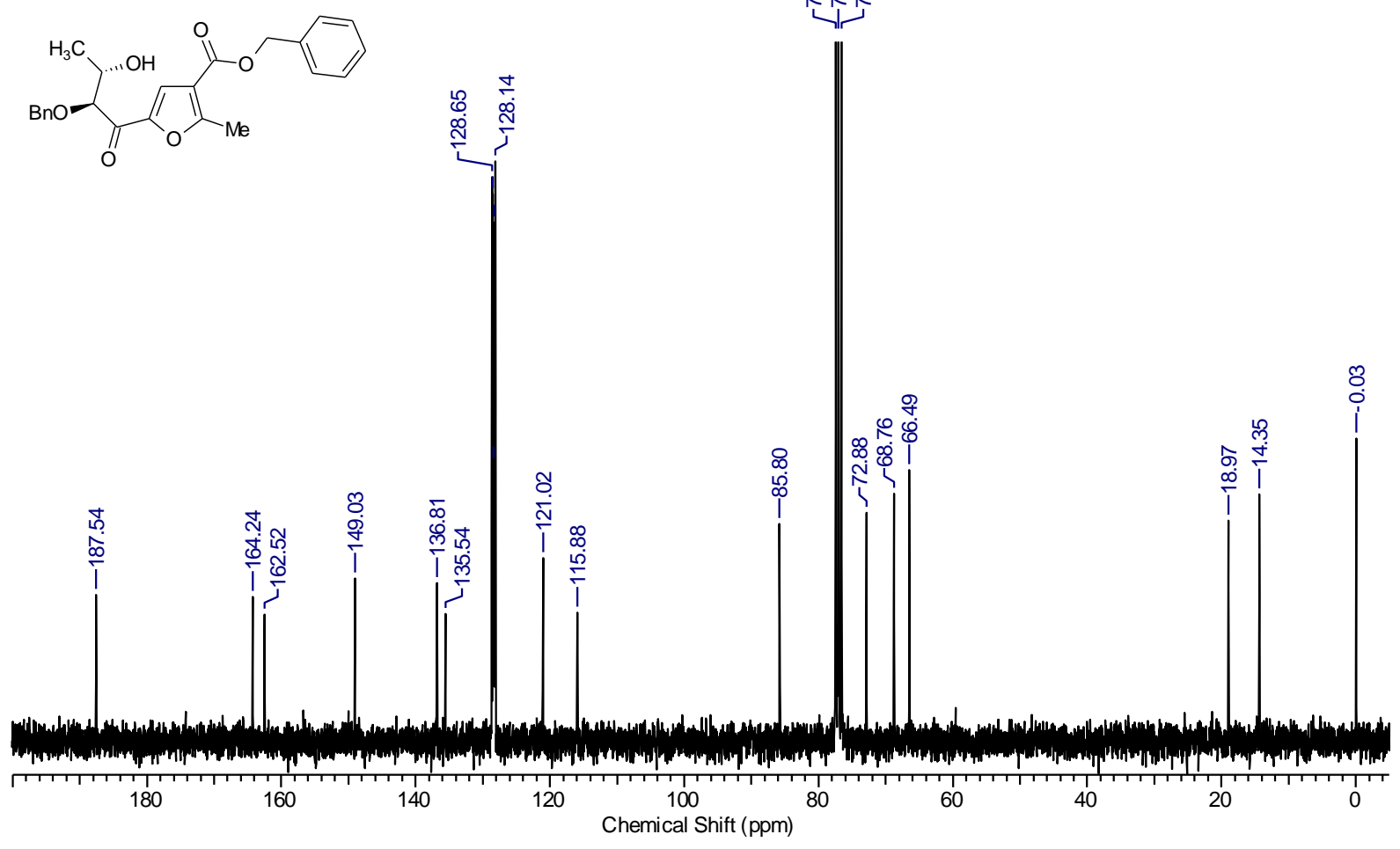


KM-487-C.002.001.1r.esp_KM-487-DEPT-135.esp

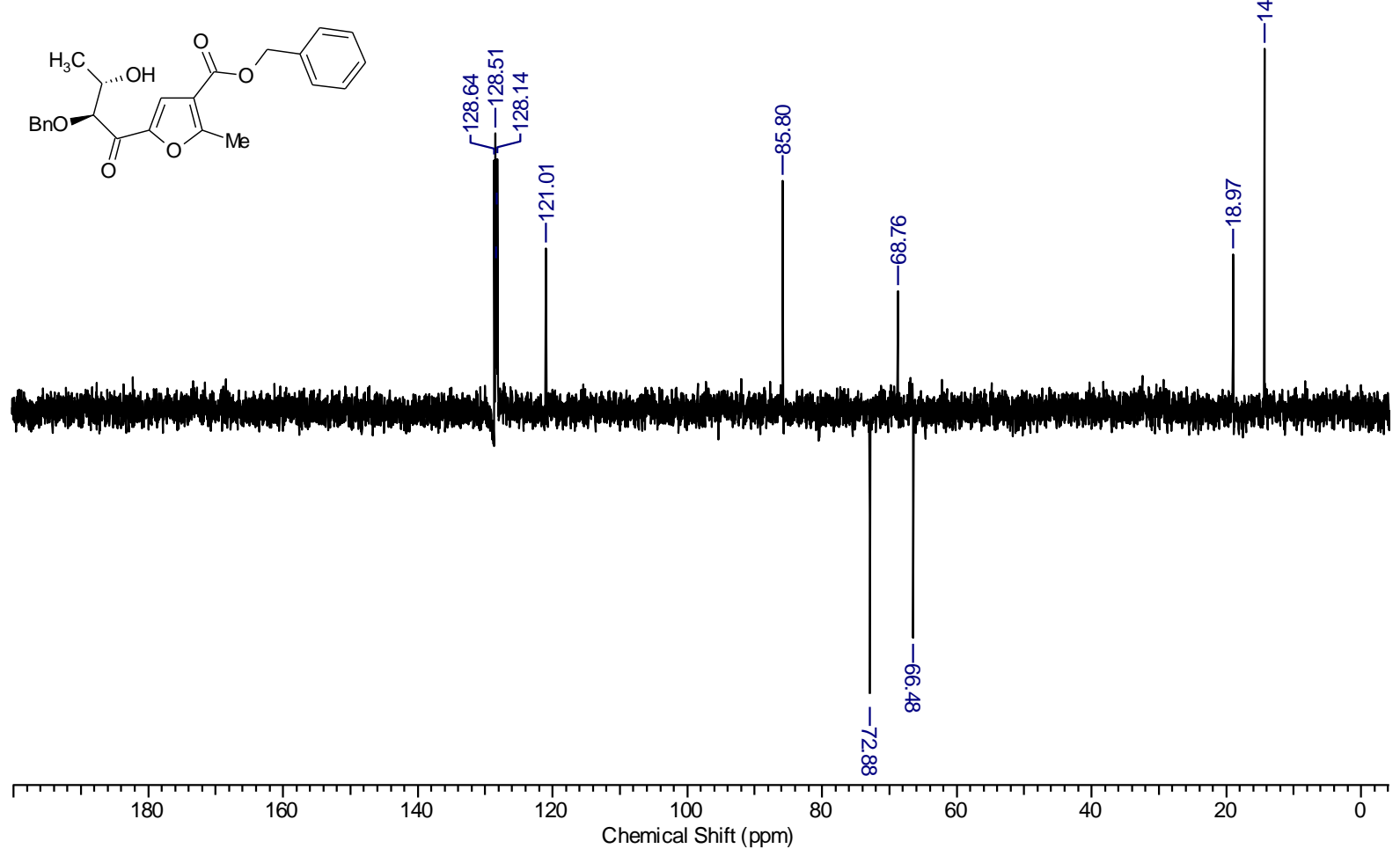

${ }^{1} \mathrm{H},{ }^{13} \mathrm{C}$, DEPT-135 NMR spectra of $3 \mathrm{j}$ :

KM-445-1H.001.001.1r.esp_1H.esp

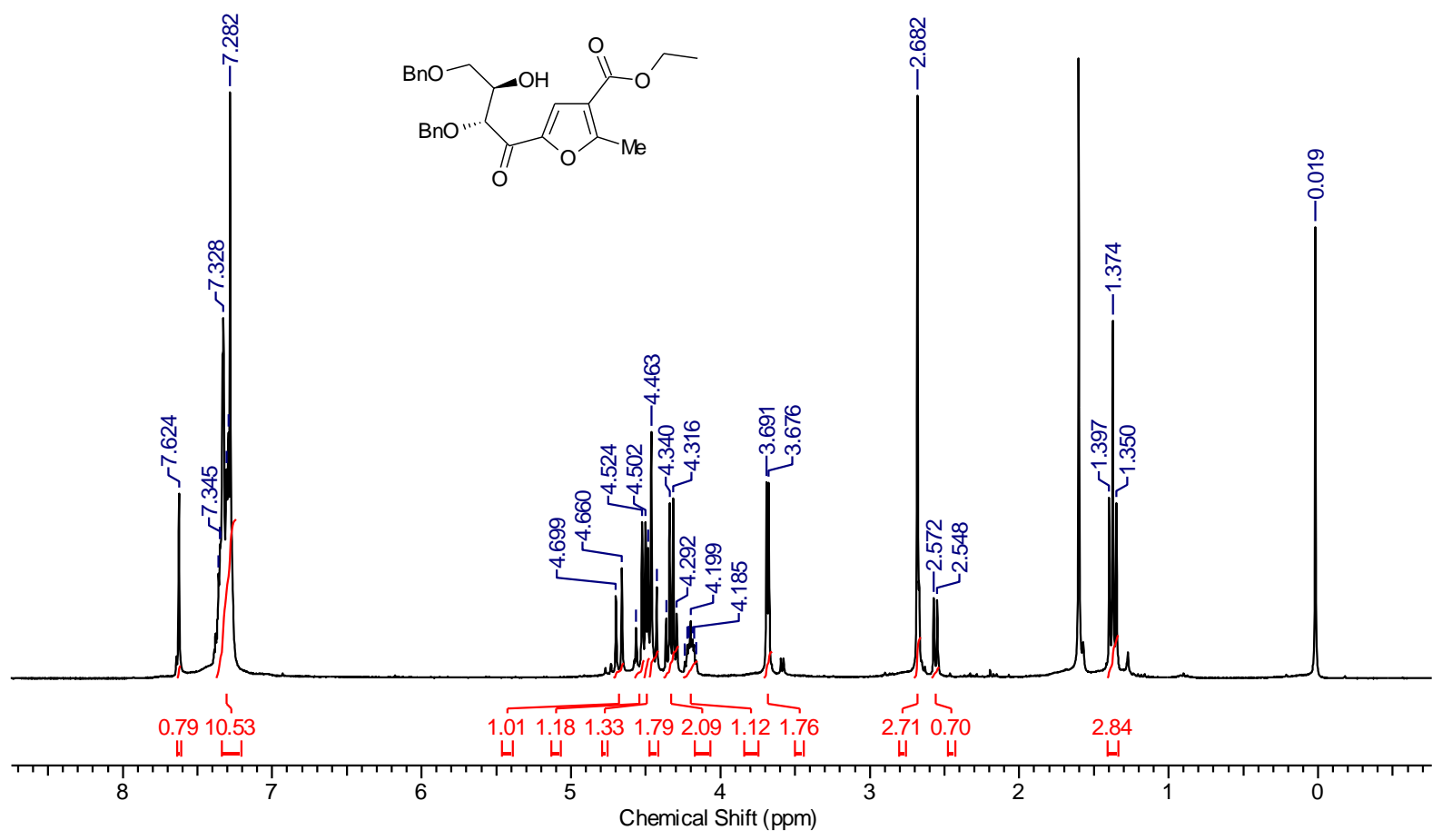



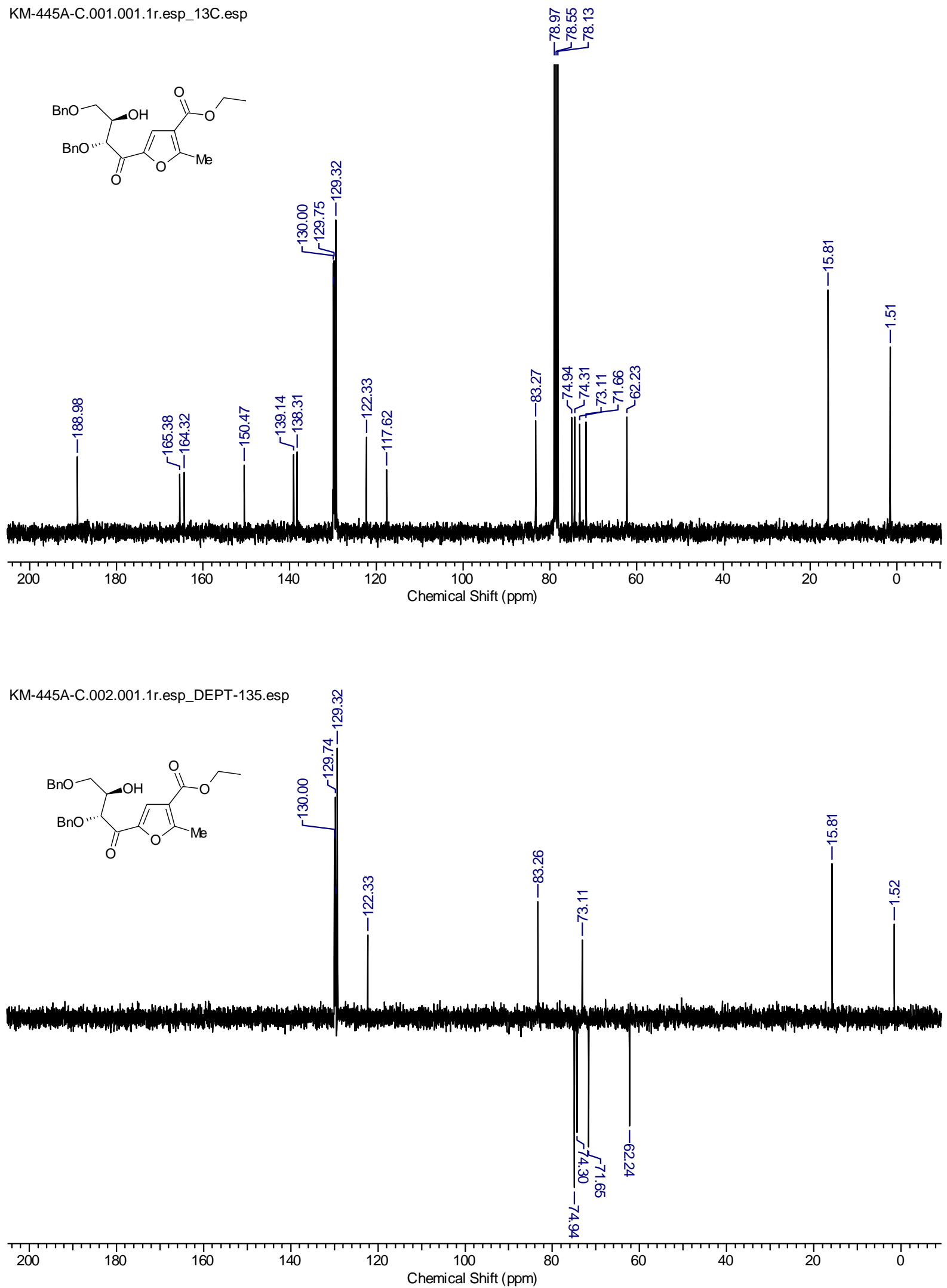


\section{${ }^{1} \mathrm{H},{ }^{13} \mathrm{C}$, DEPT-135 NMR spectra of 3k:}

ID-0815.081.001.1r_KM-458-1H.esp<smiles>COC(=O)c1cc(C(=O)[C@@H](O)[C@H](O)COc2ccccc2)oc1[N+](=O)[O-]</smiles>

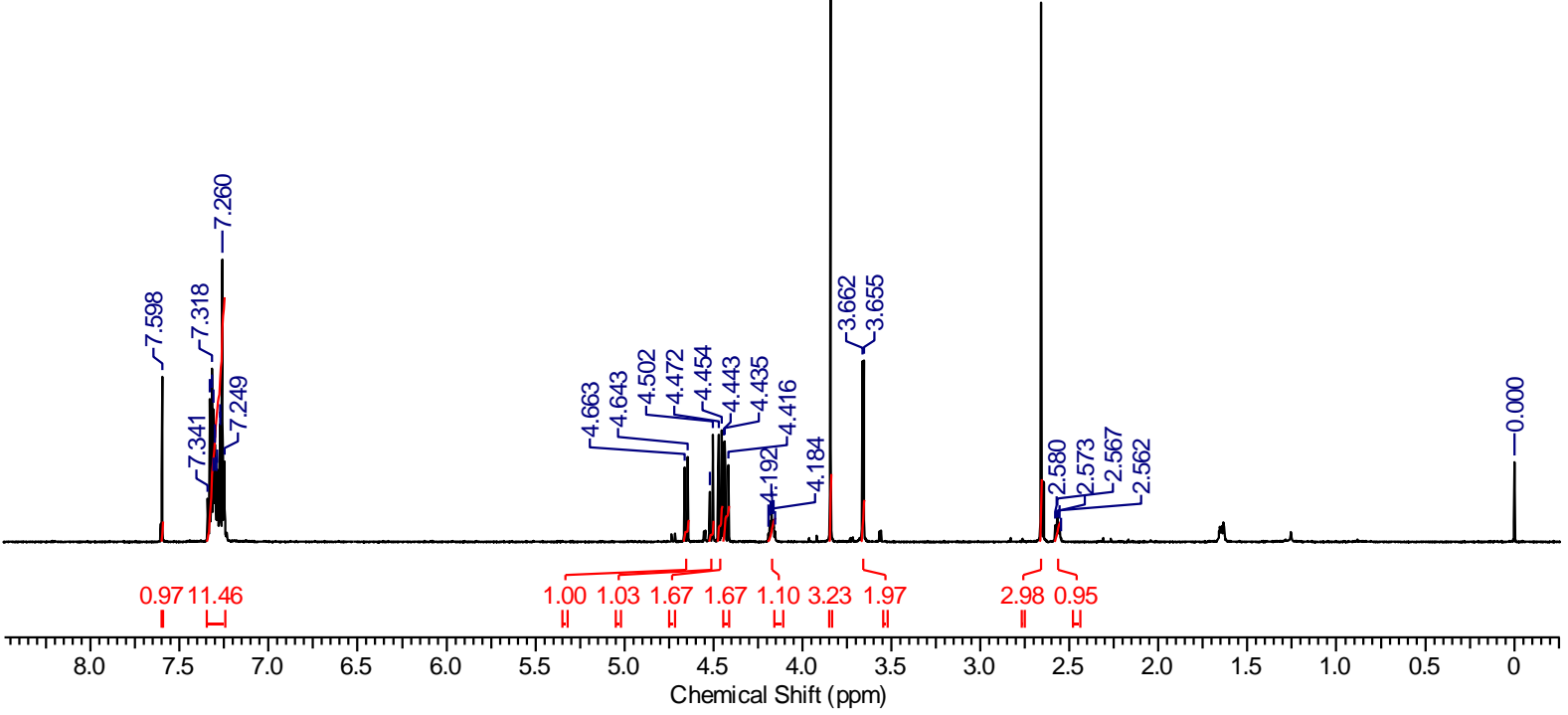

ID-0815.082.001.1r_KM-458-13C.esp<smiles>COC(=O)c1cc(C(=O)[C@@H](O)[C@H](O)COc2ccccc2)oc1[N+](=O)[O-]</smiles>

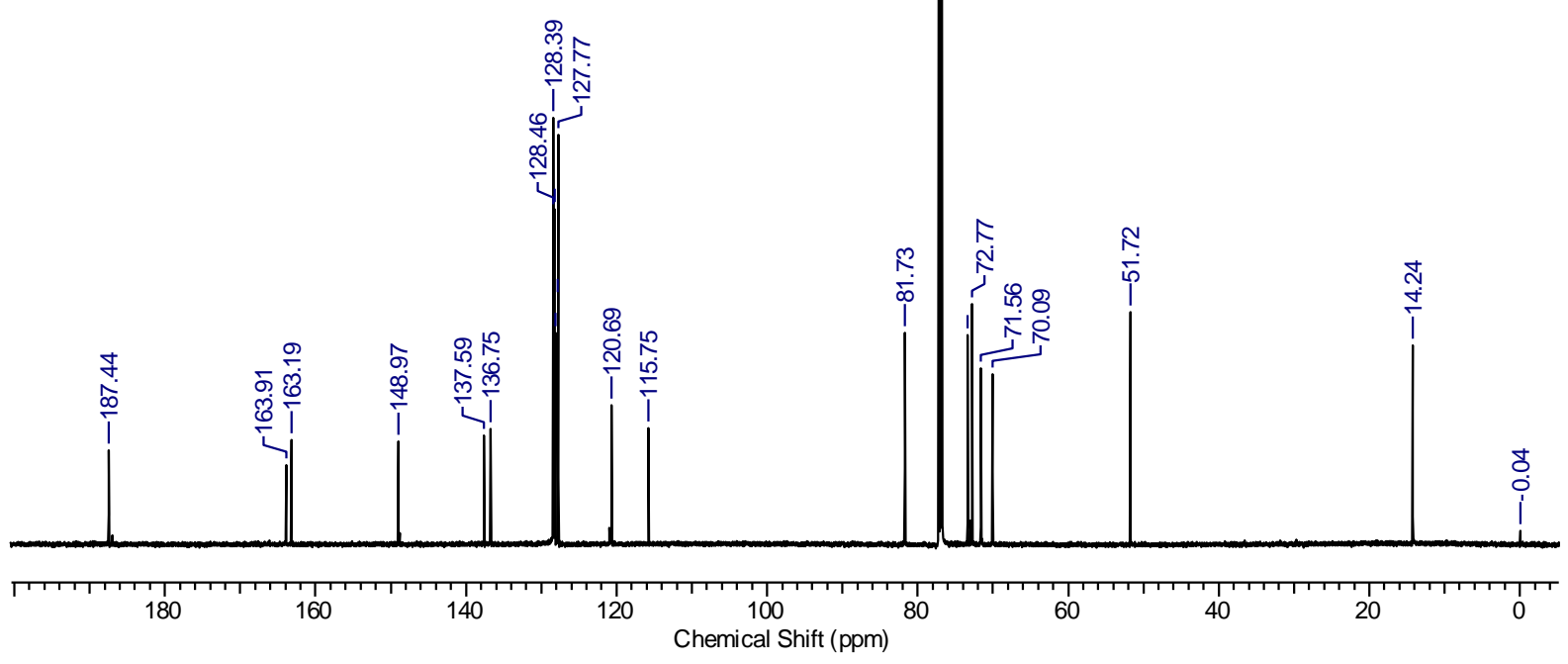




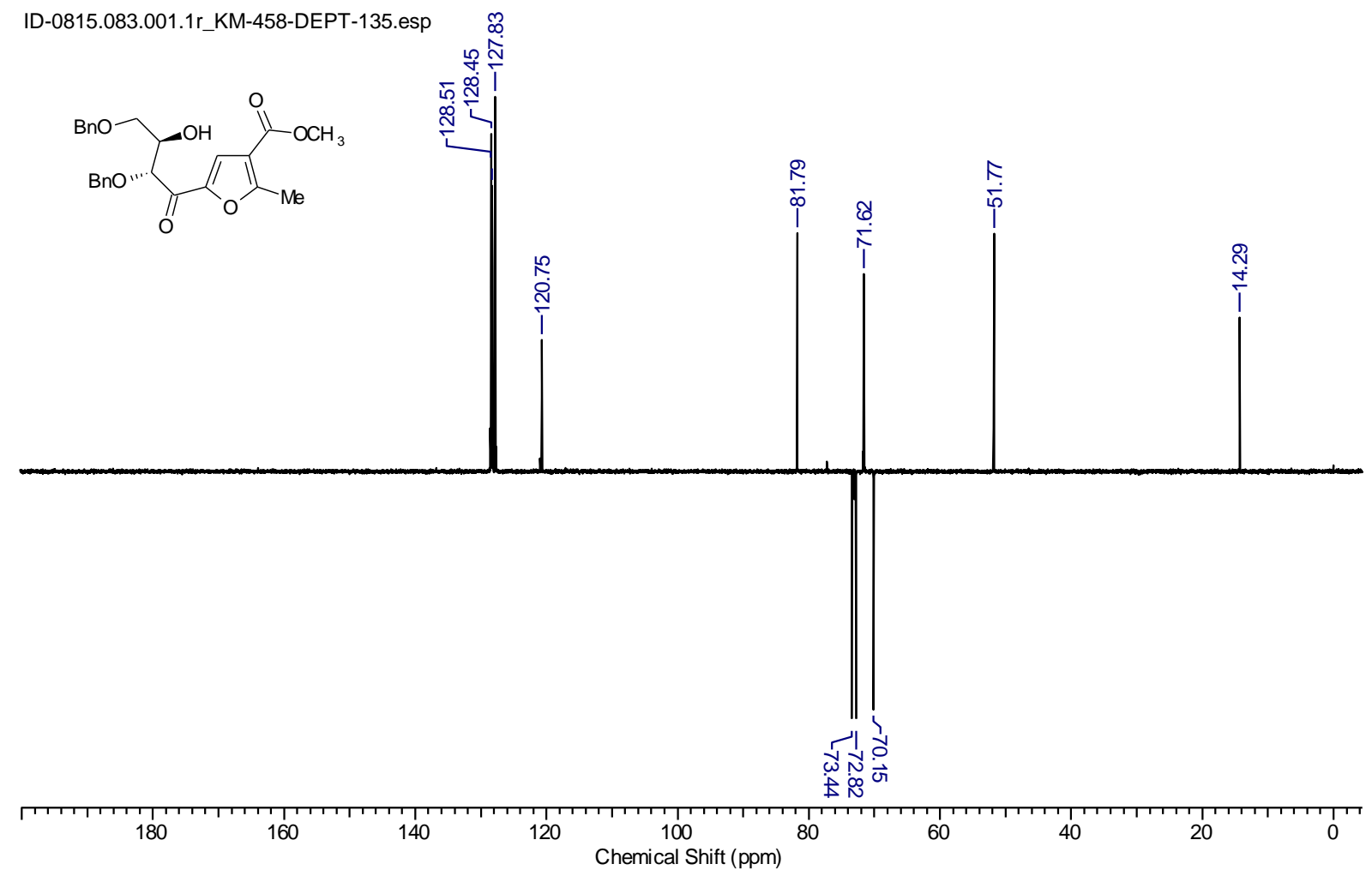

2D (COSY, HMBC, HSQC, and NOESY) NMR spectra of 3k:

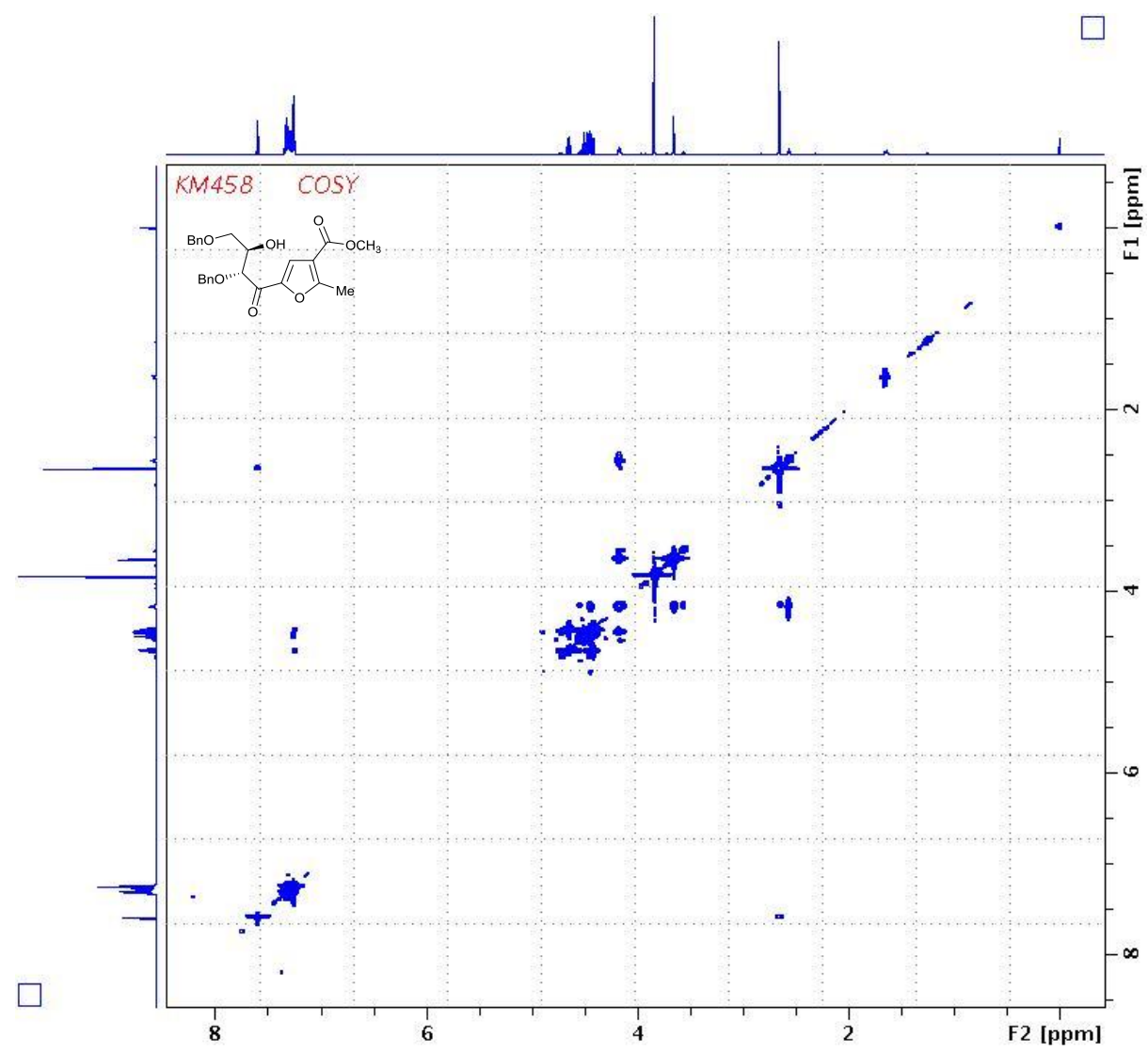



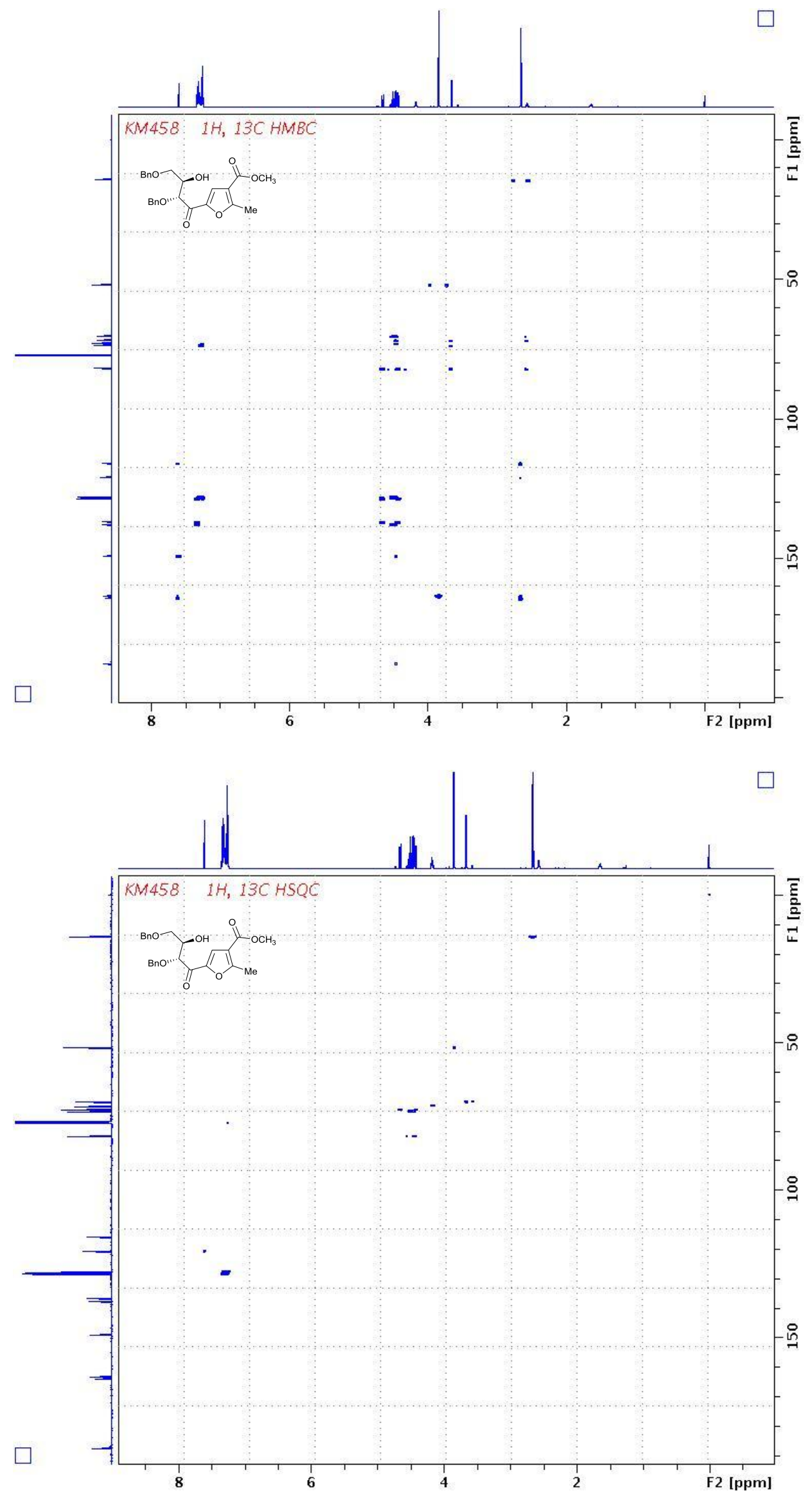


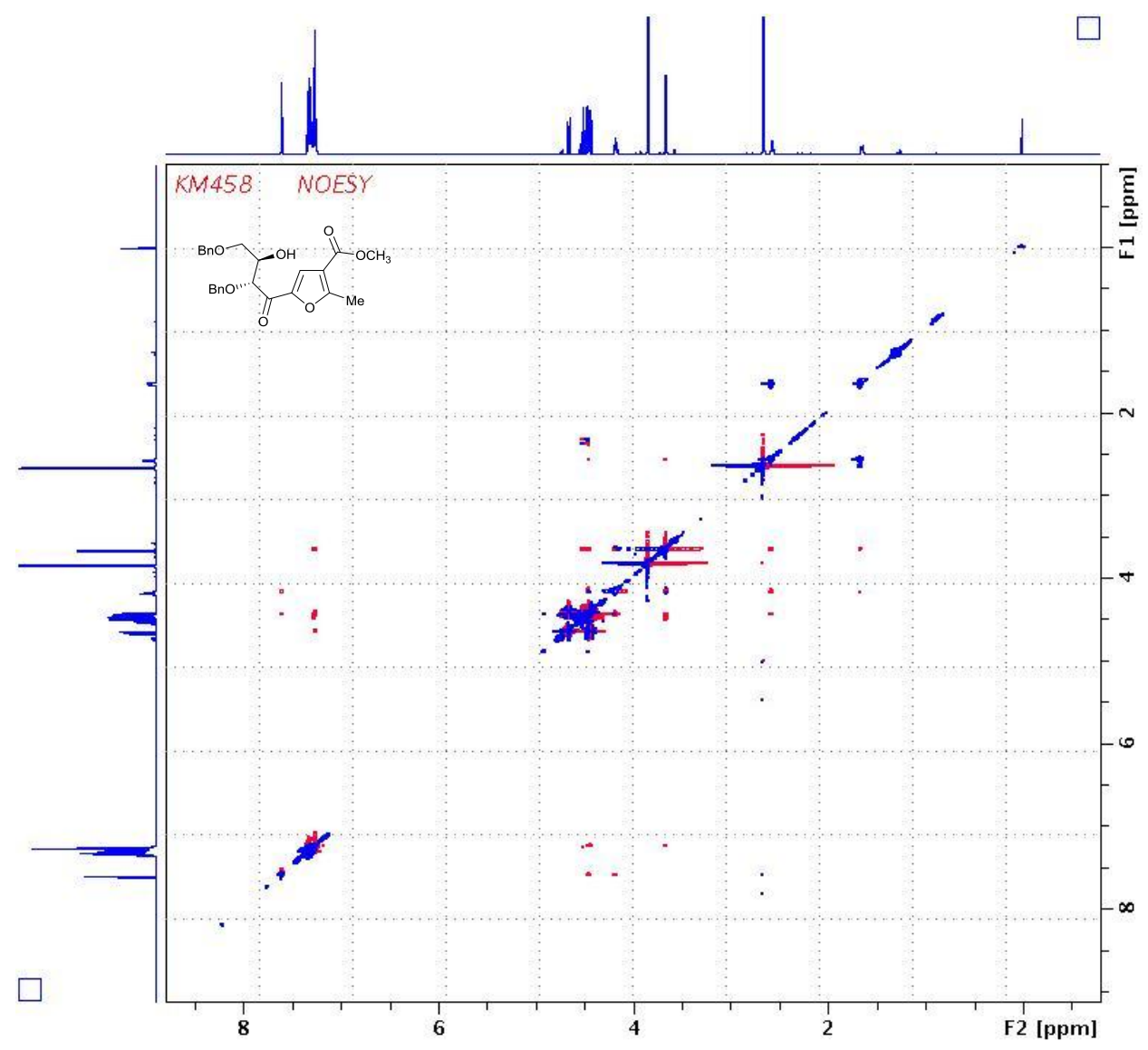

${ }^{1} \mathrm{H},{ }^{13} \mathrm{C}$, DEPT-135 NMR spectra of 3l:

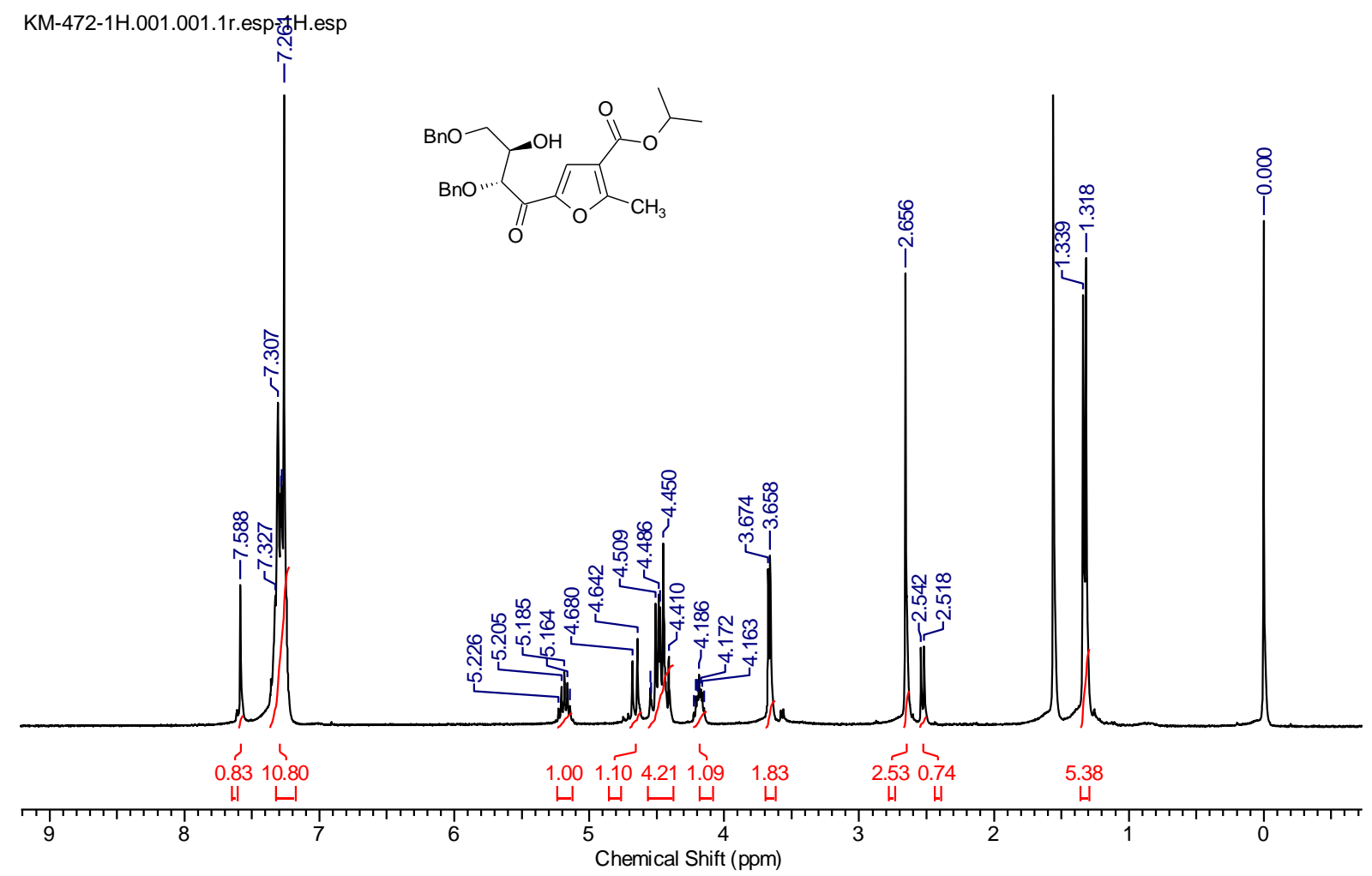




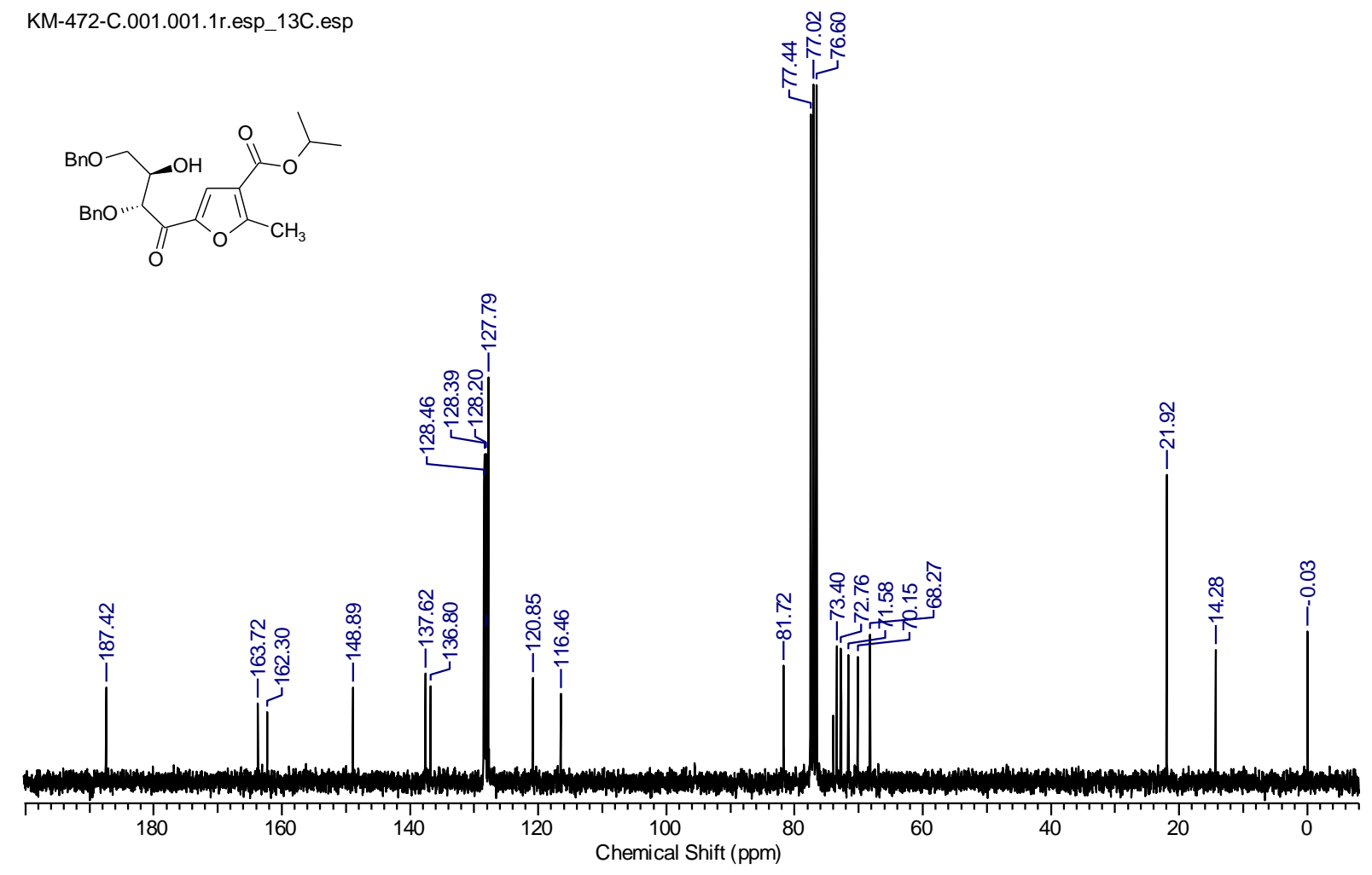

KM-472-C.002.001.1r.esp_DEPT-135.esp
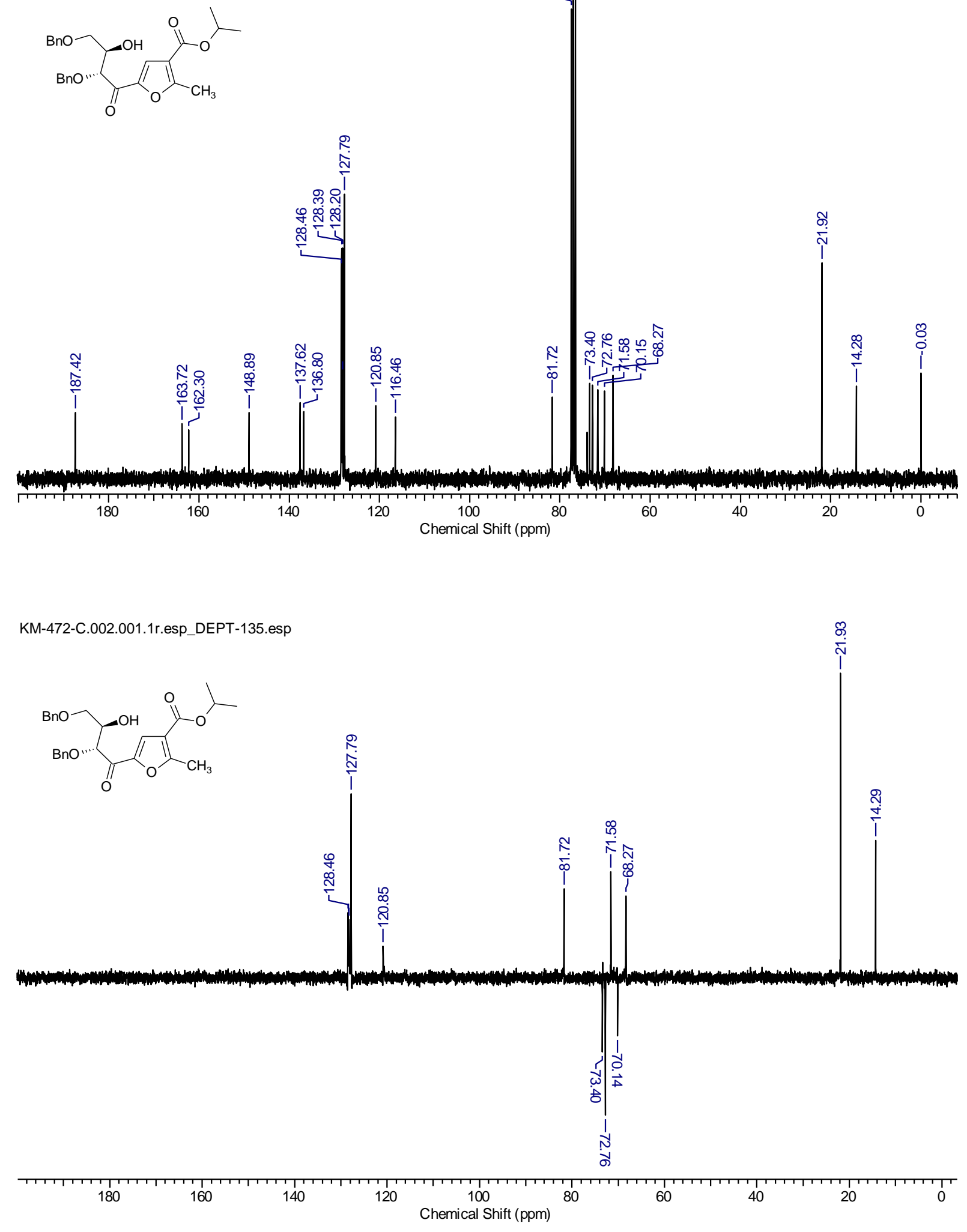
${ }^{1} \mathrm{H},{ }^{13} \mathrm{C}$, DEPT-135 NMR spectra of $3 \mathrm{~m}$ :

KM-454-1H.001.001.1r.esp_1H.esp

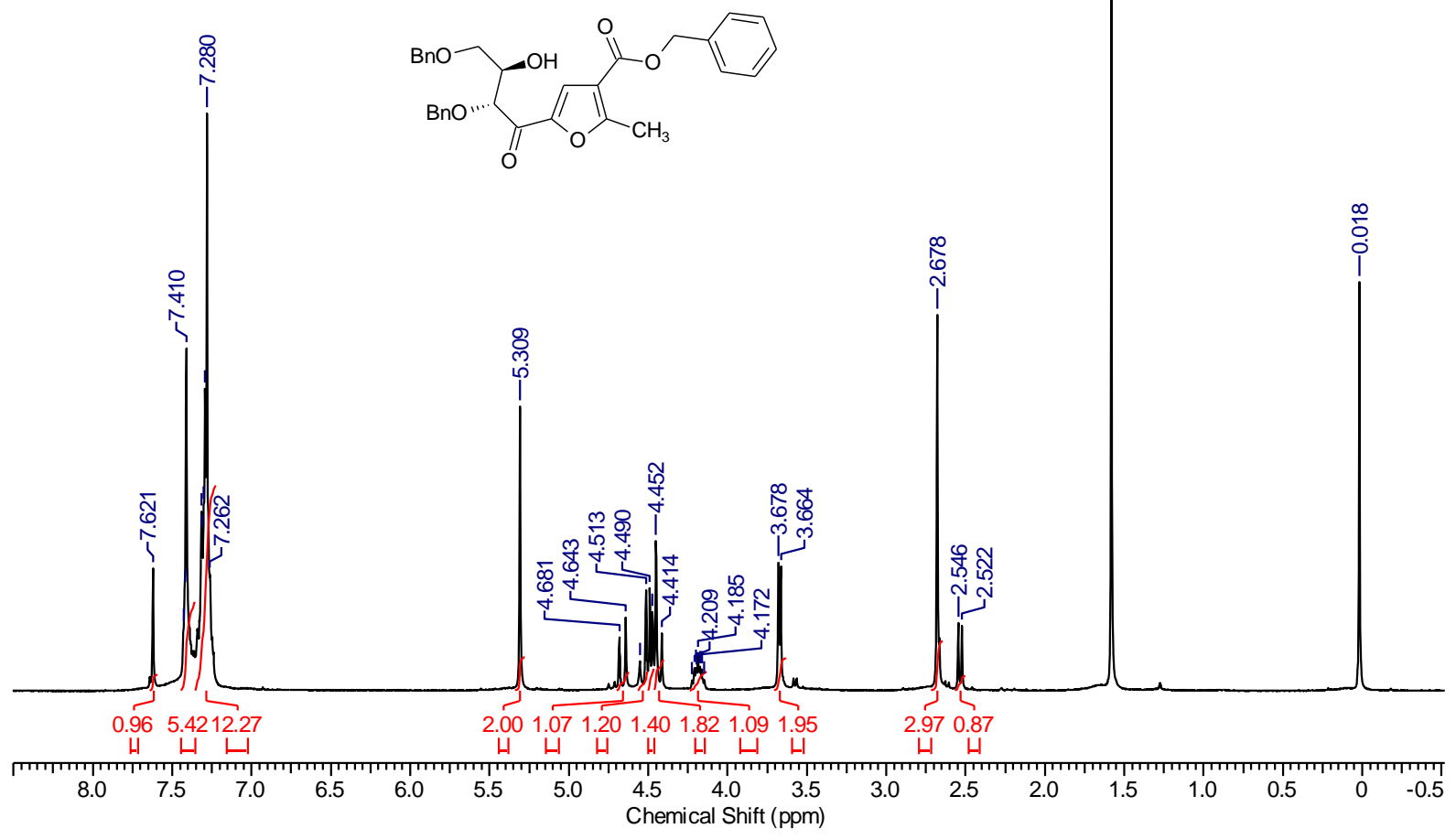

KM-454-C.001.001.1r.esp_13C.esp

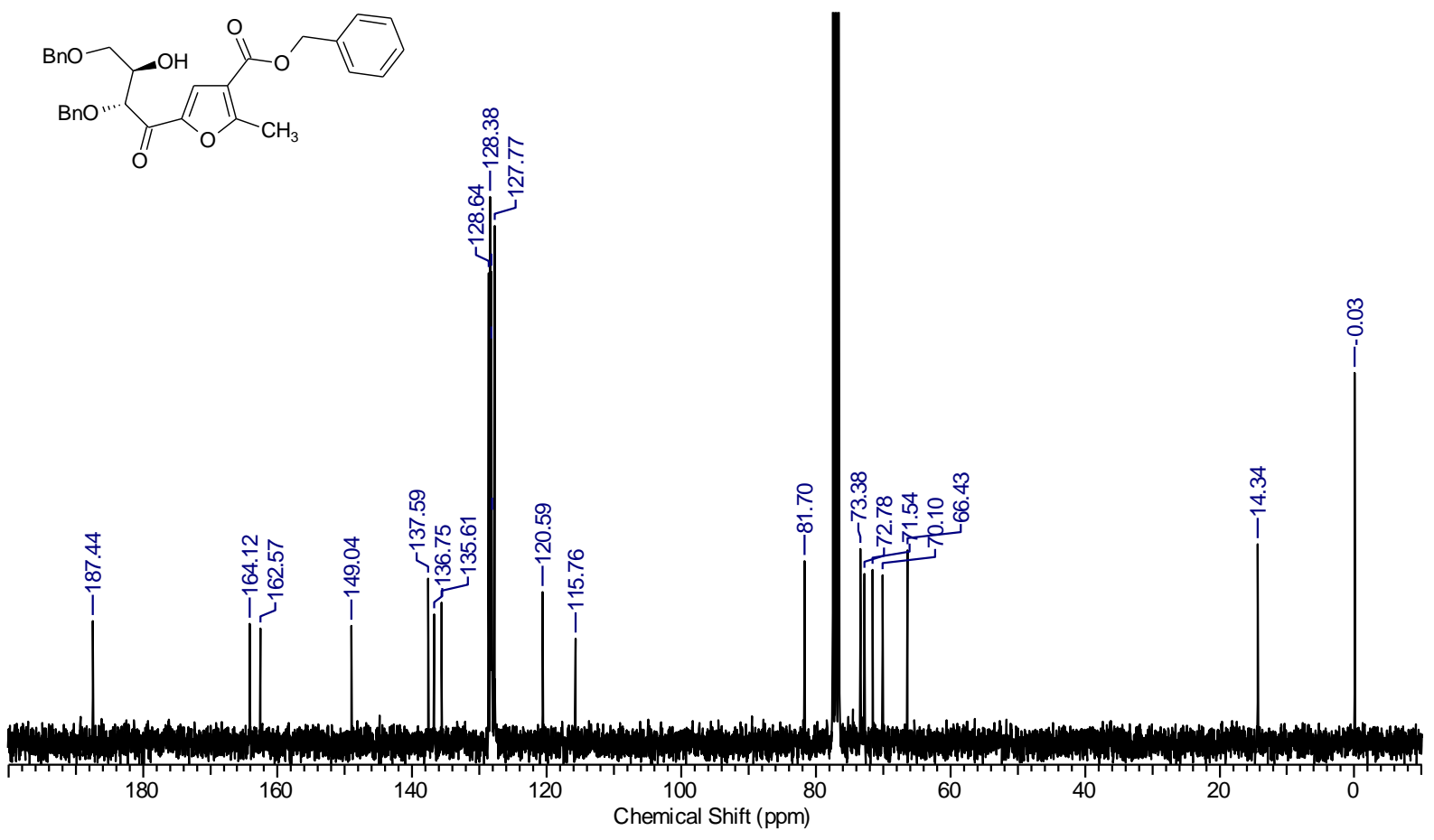




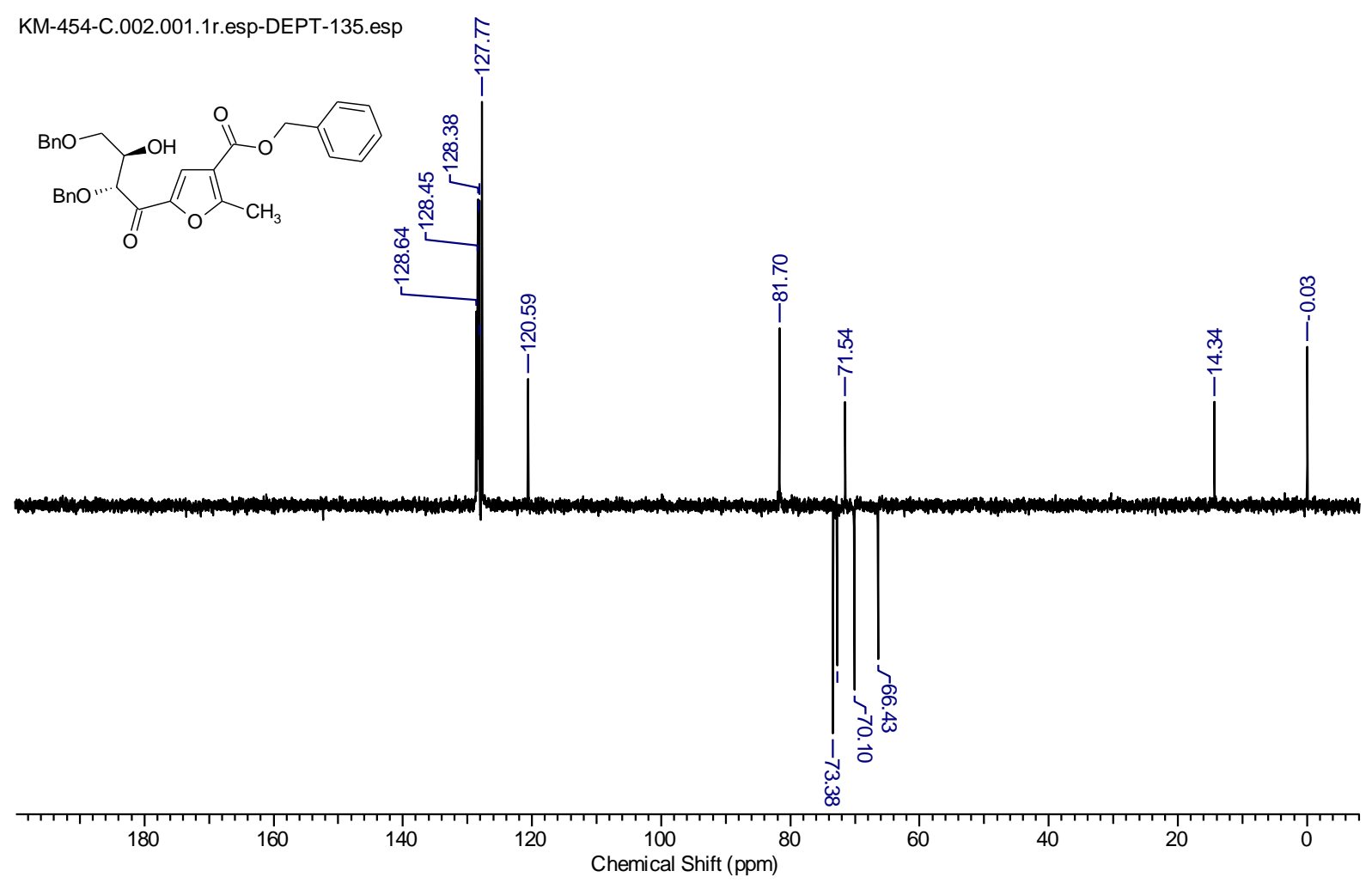

${ }^{1} \mathrm{H},{ }^{13} \mathrm{C}$, DEPT-135 NMR spectra of 3n:

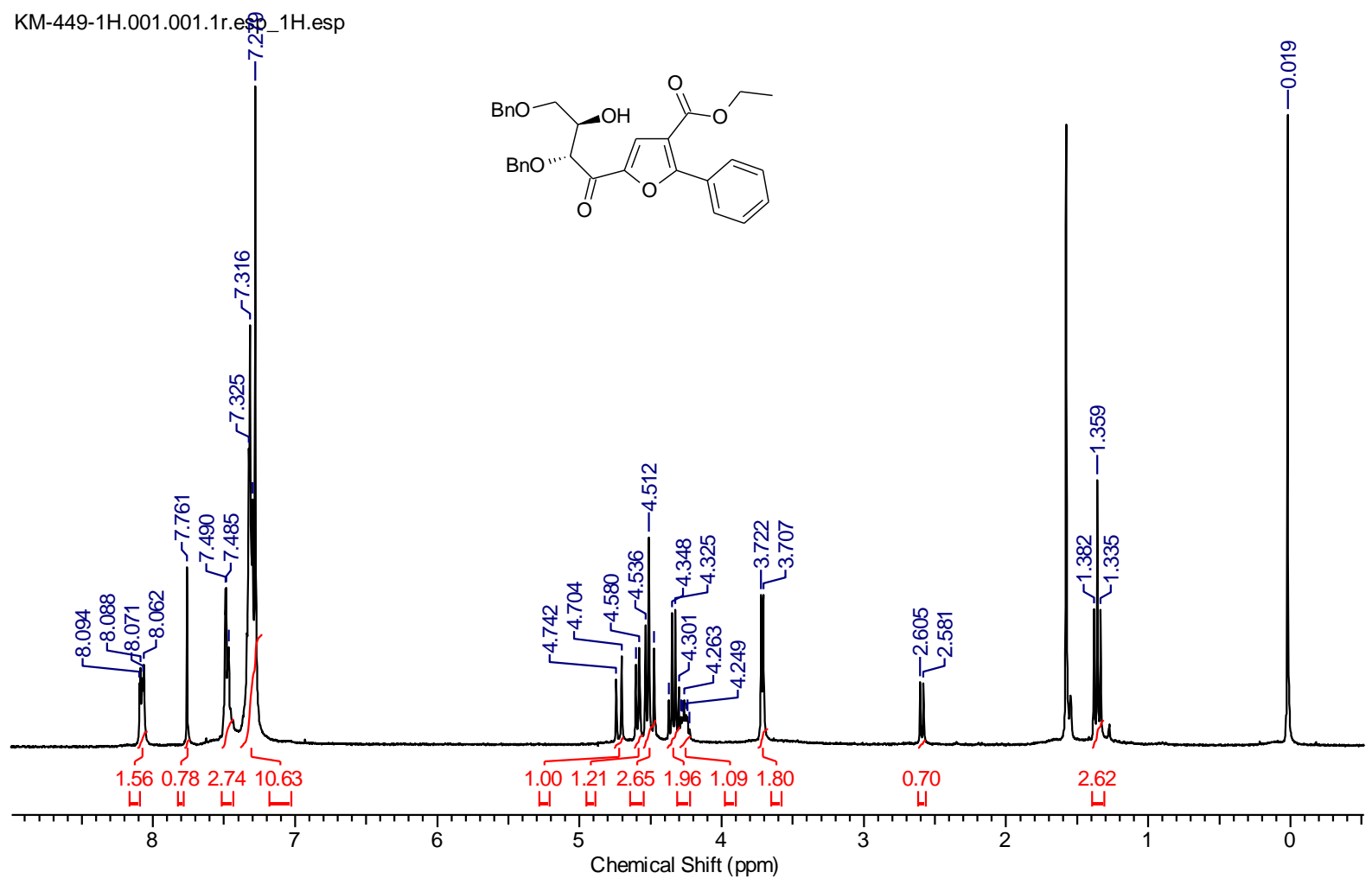

S89 

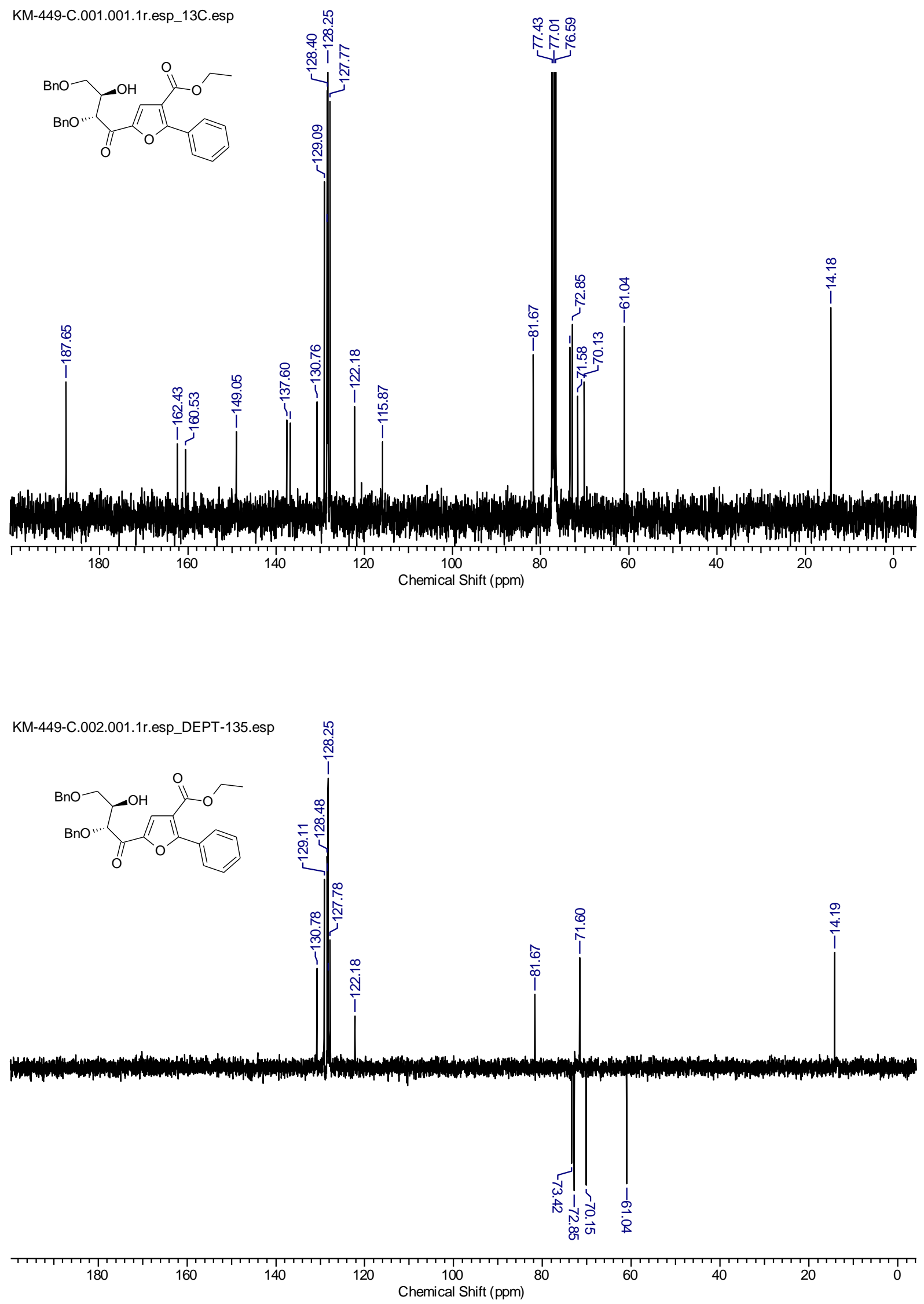

S90 


\section{${ }^{1} \mathrm{H},{ }^{13} \mathrm{C}$, DEPT-135 NMR spectra of 3o:}

KM-447.001.001.1r.esp-1H.esp<smiles>CCOC(=O)c1cc(C(=O)[C@@H](O)[C@H](O)COCc2ccccc2)oc1-c1ccc([N+](=O)[O-])cc1</smiles>
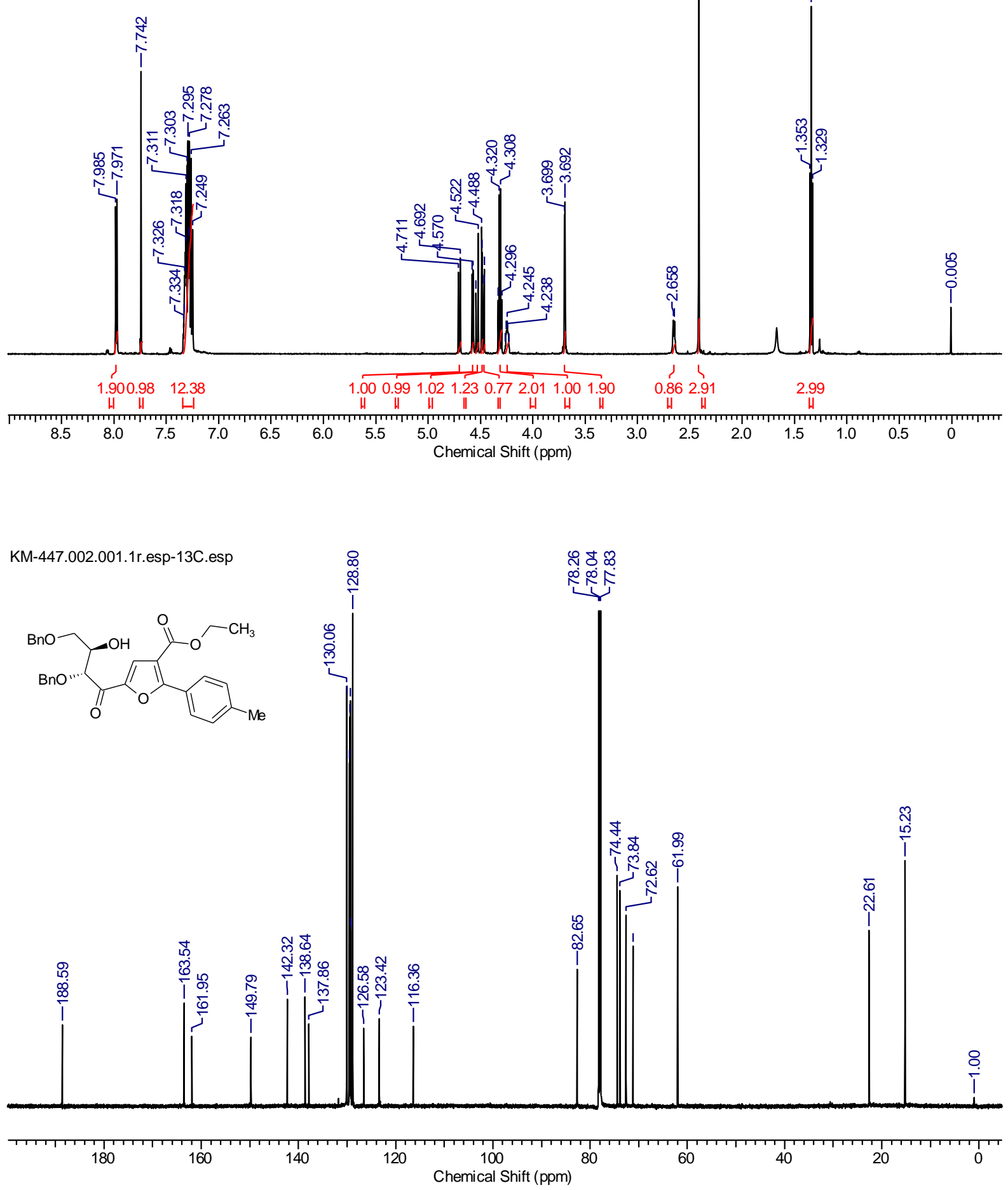


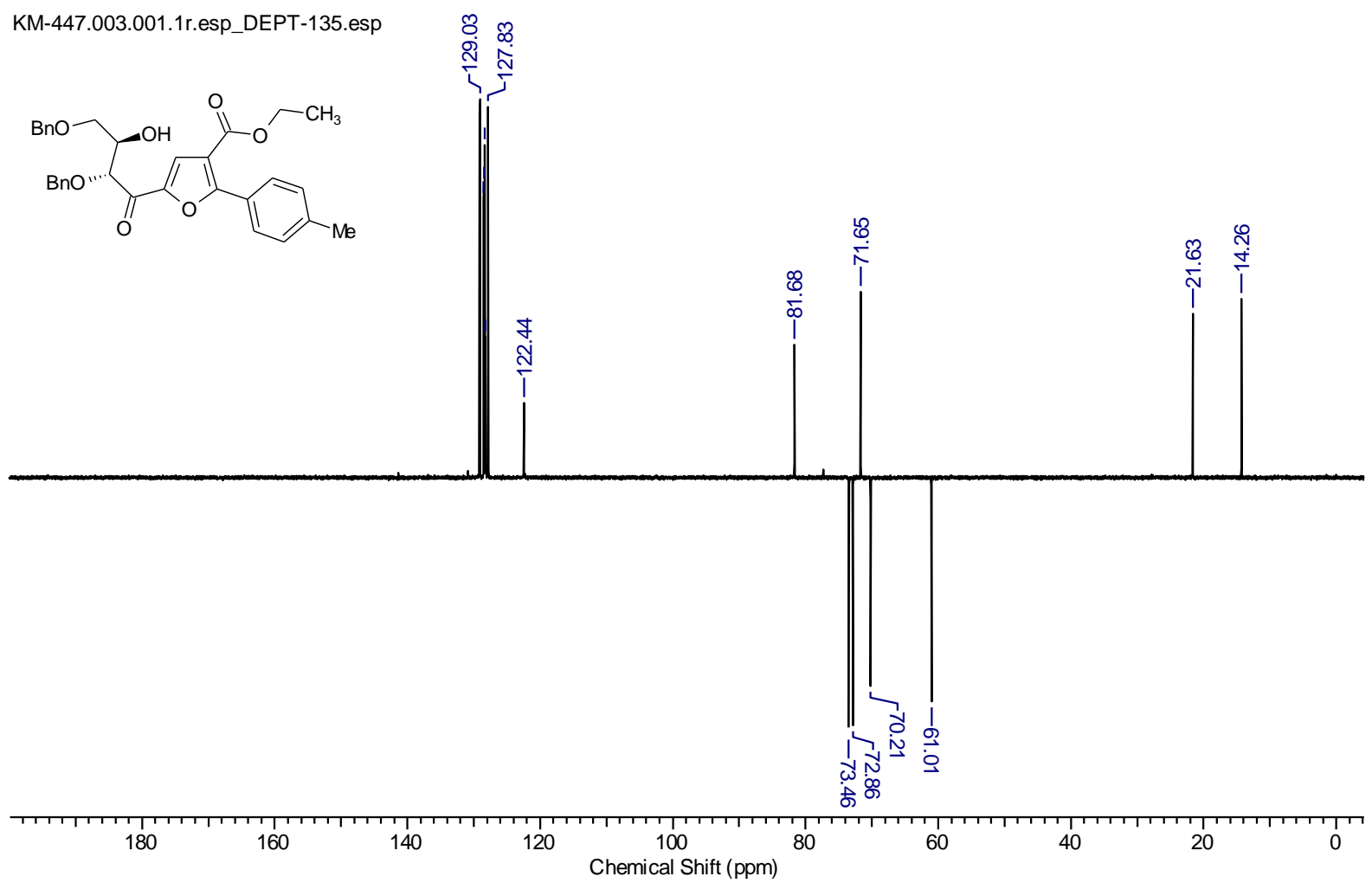

\section{${ }^{1} \mathrm{H},{ }^{13} \mathrm{C}$, DEPT-135 NMR spectra of 3p:}

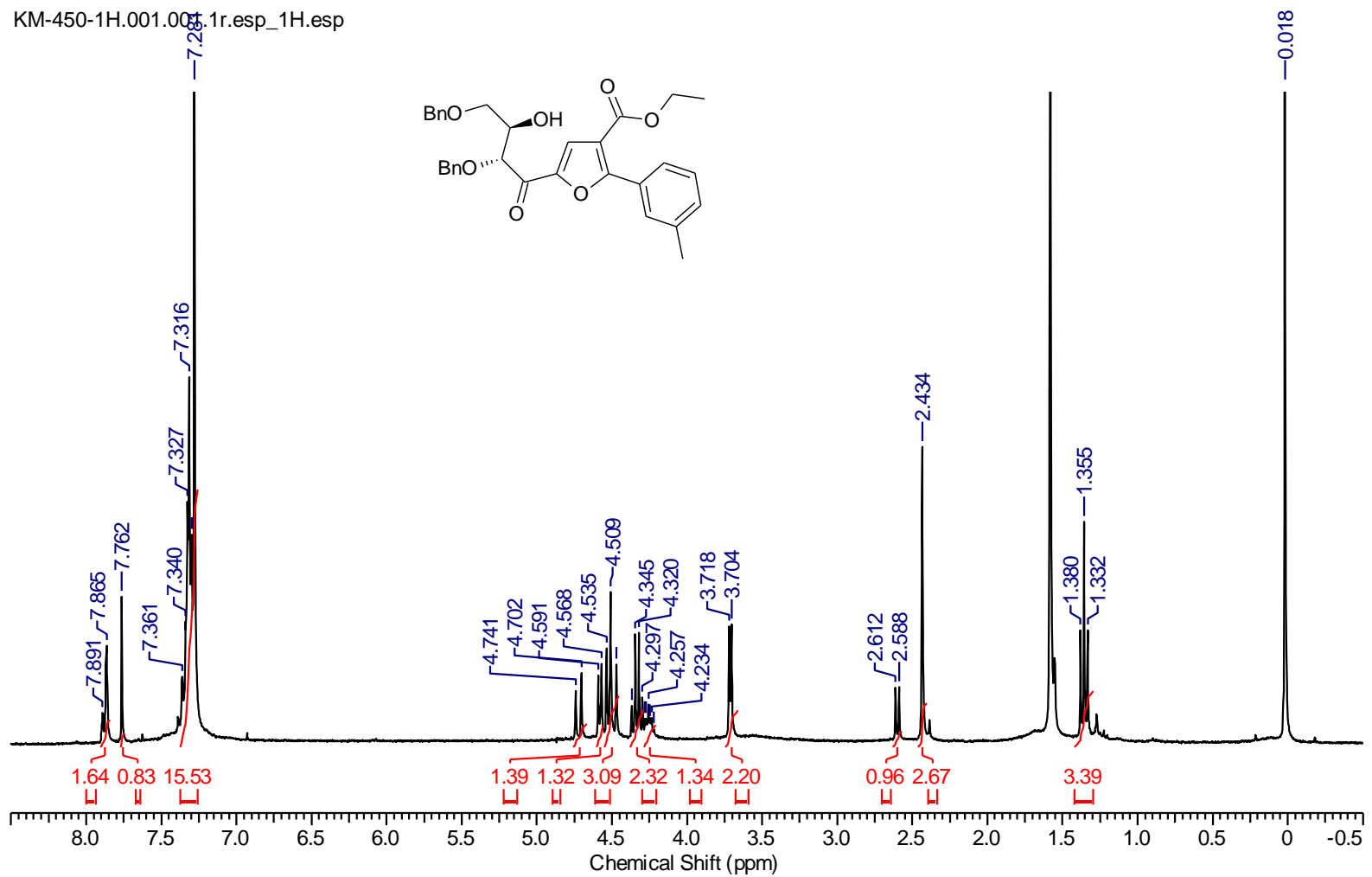



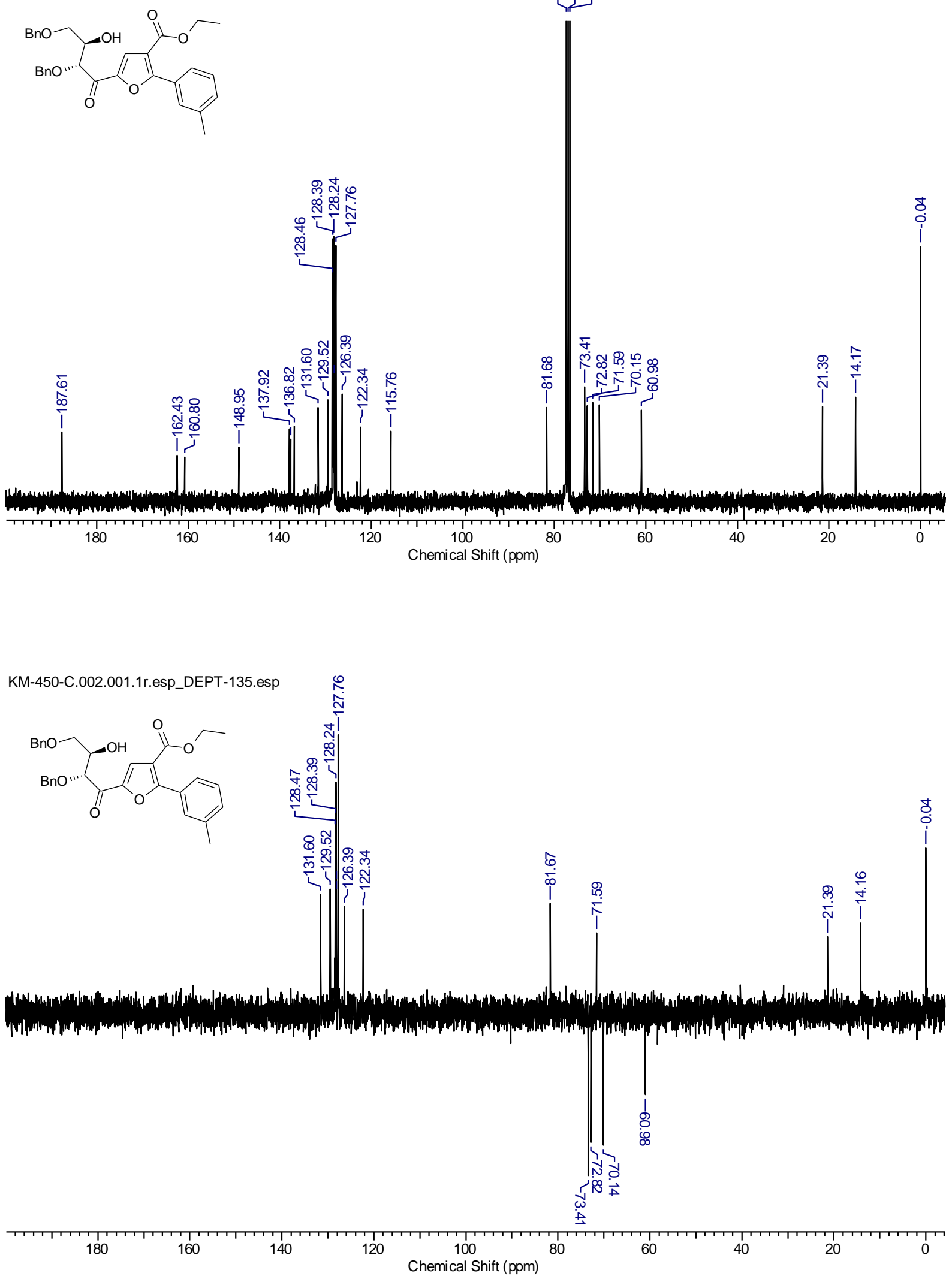
${ }^{1} \mathrm{H},{ }^{13} \mathrm{C}$, DEPT-135 NMR spectra of 3q:
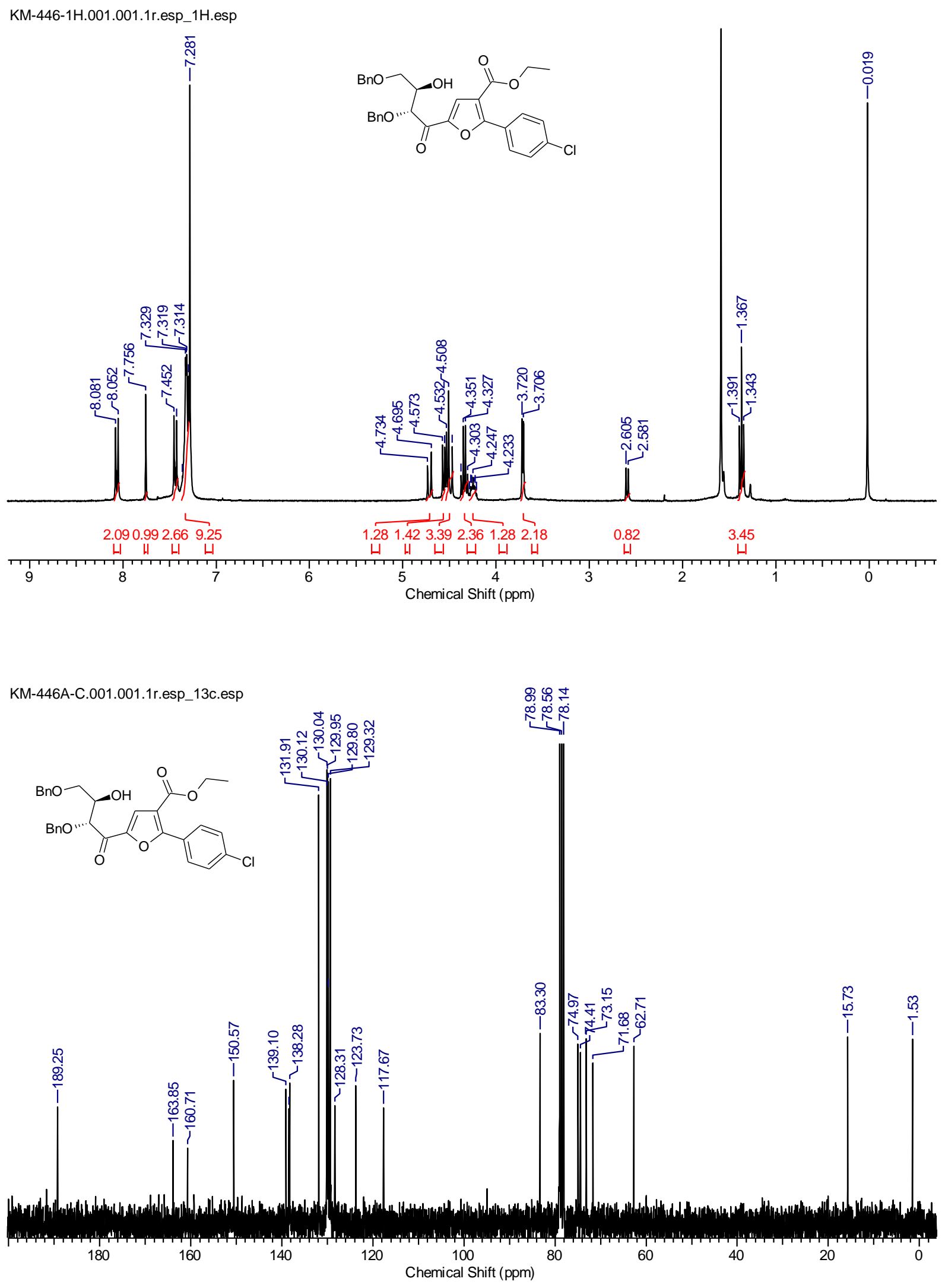

S94 


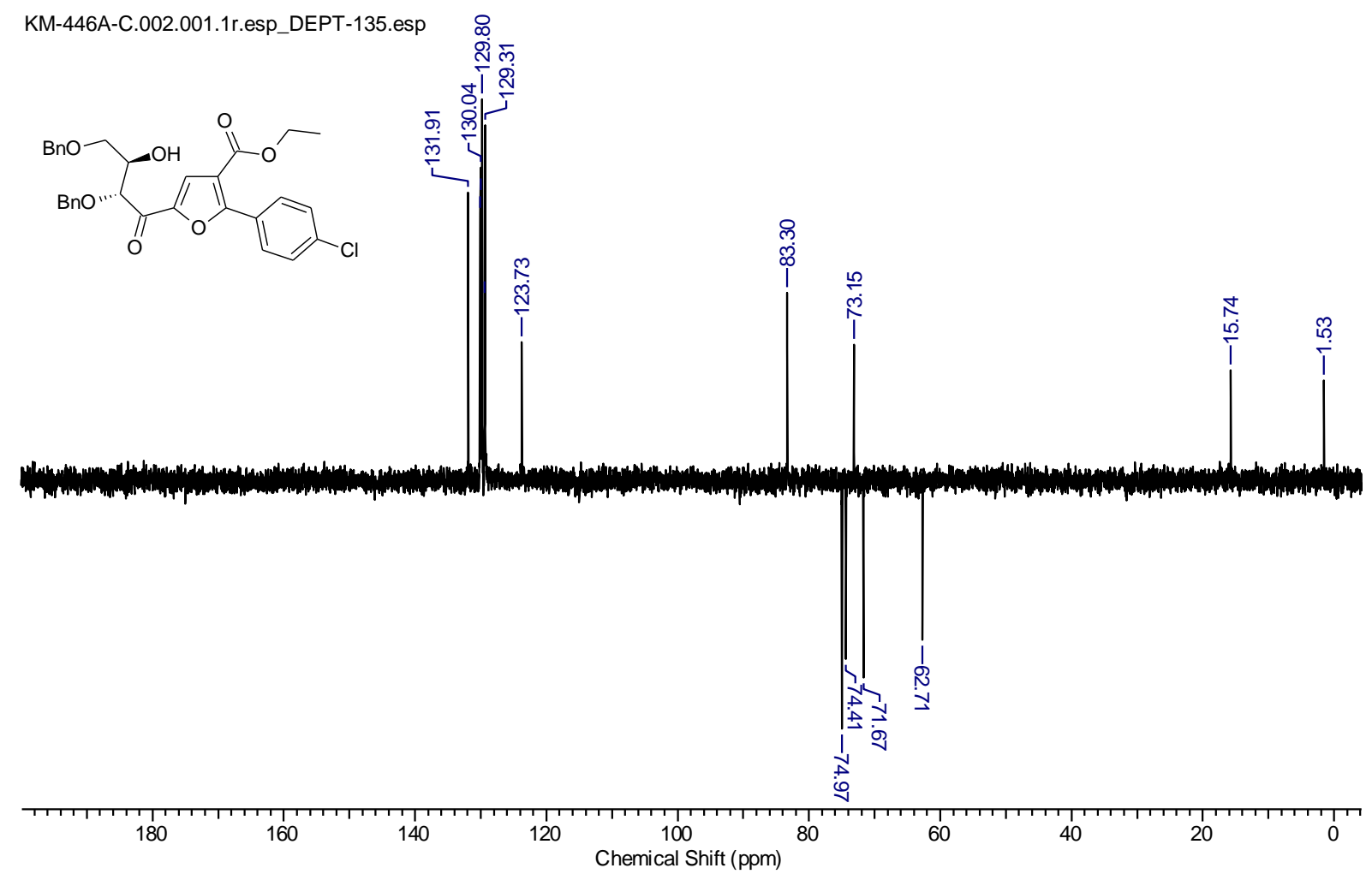

${ }^{1} \mathrm{H},{ }^{13} \mathrm{C}$, DEPT-135 NMR spectra of 3r:

ID-0815.021.001.1r_KM-470-1H.esp<smiles>COC(=O)c1cc(C(=O)[C@@H](O)[C@H](O)COc2ccccc2)oc1OC</smiles>
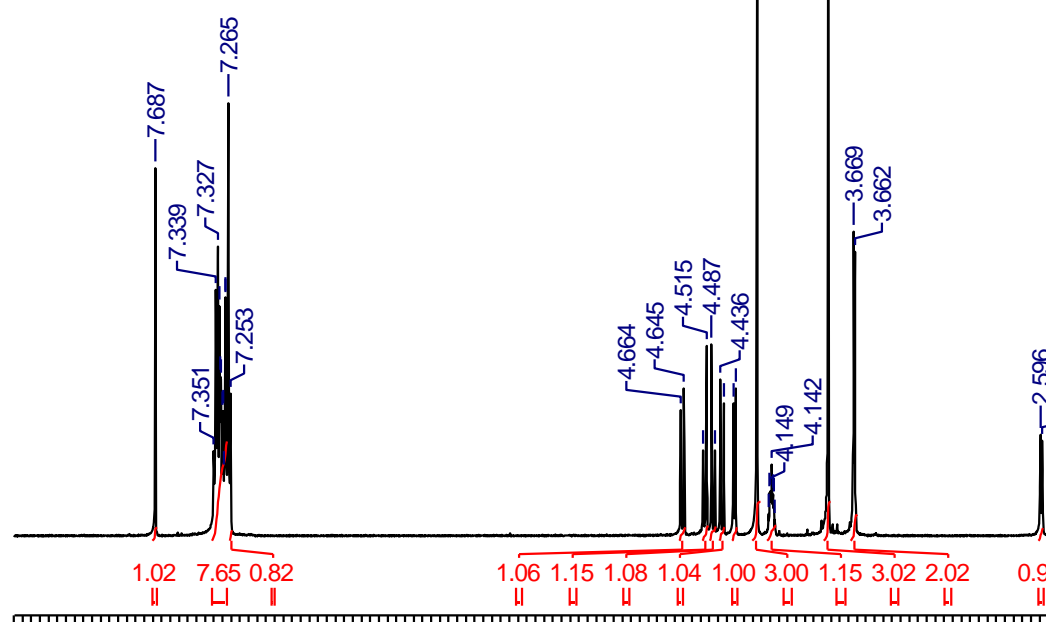

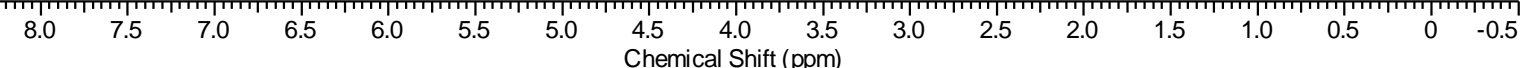



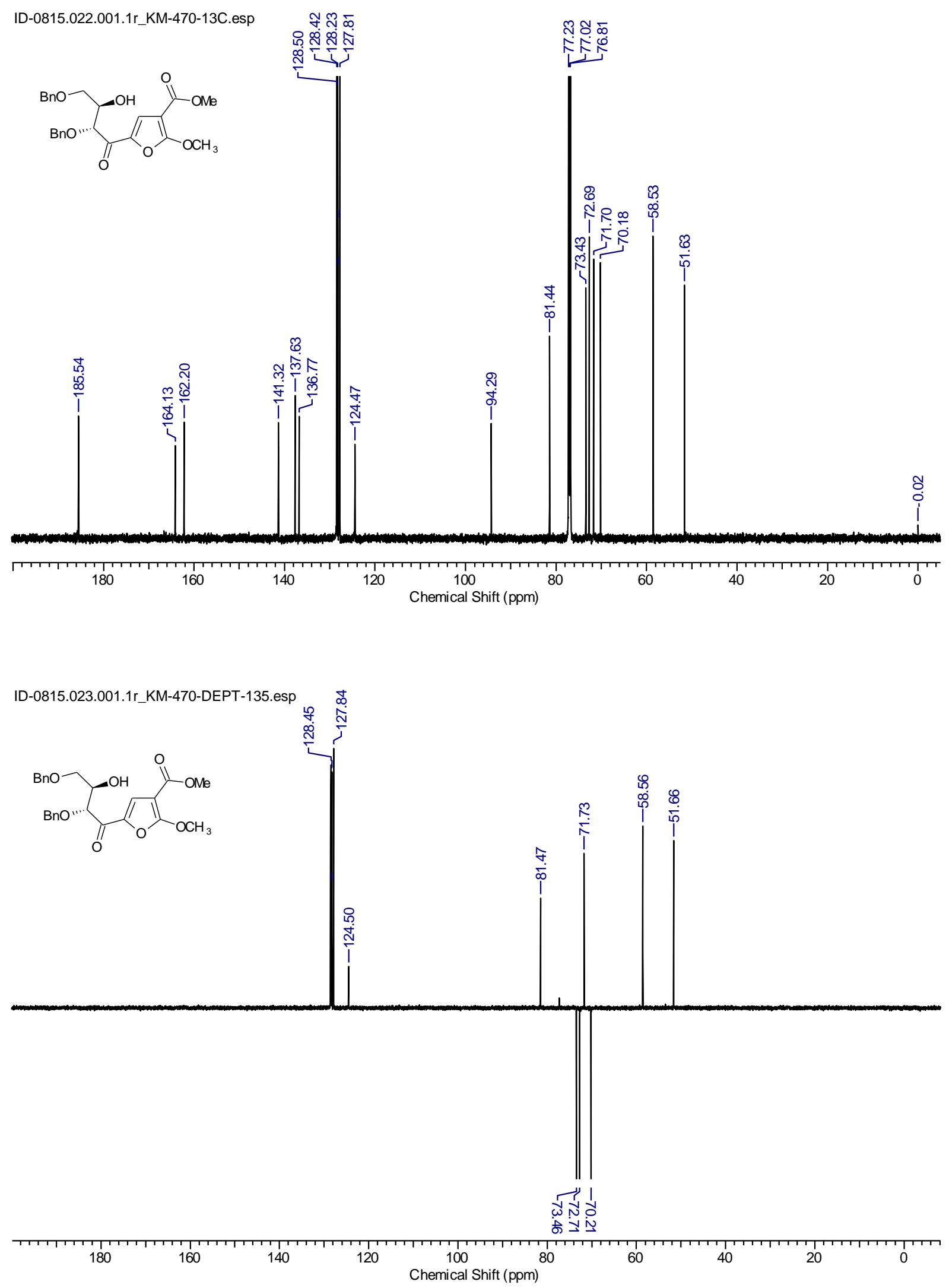

S96 
2D (COSY, HMBC, HSQC, and NOESY) NMR spectra of 3r:
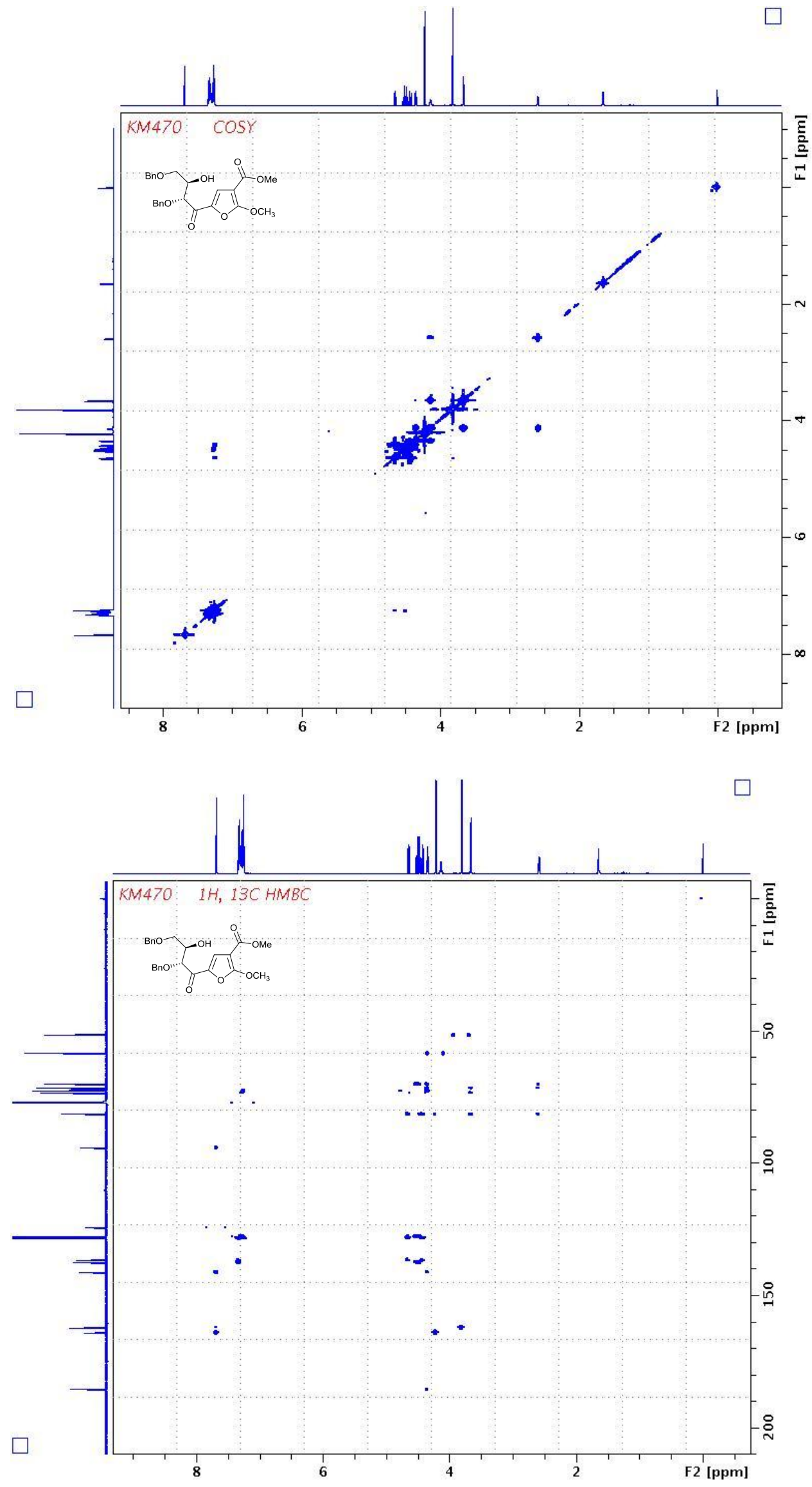

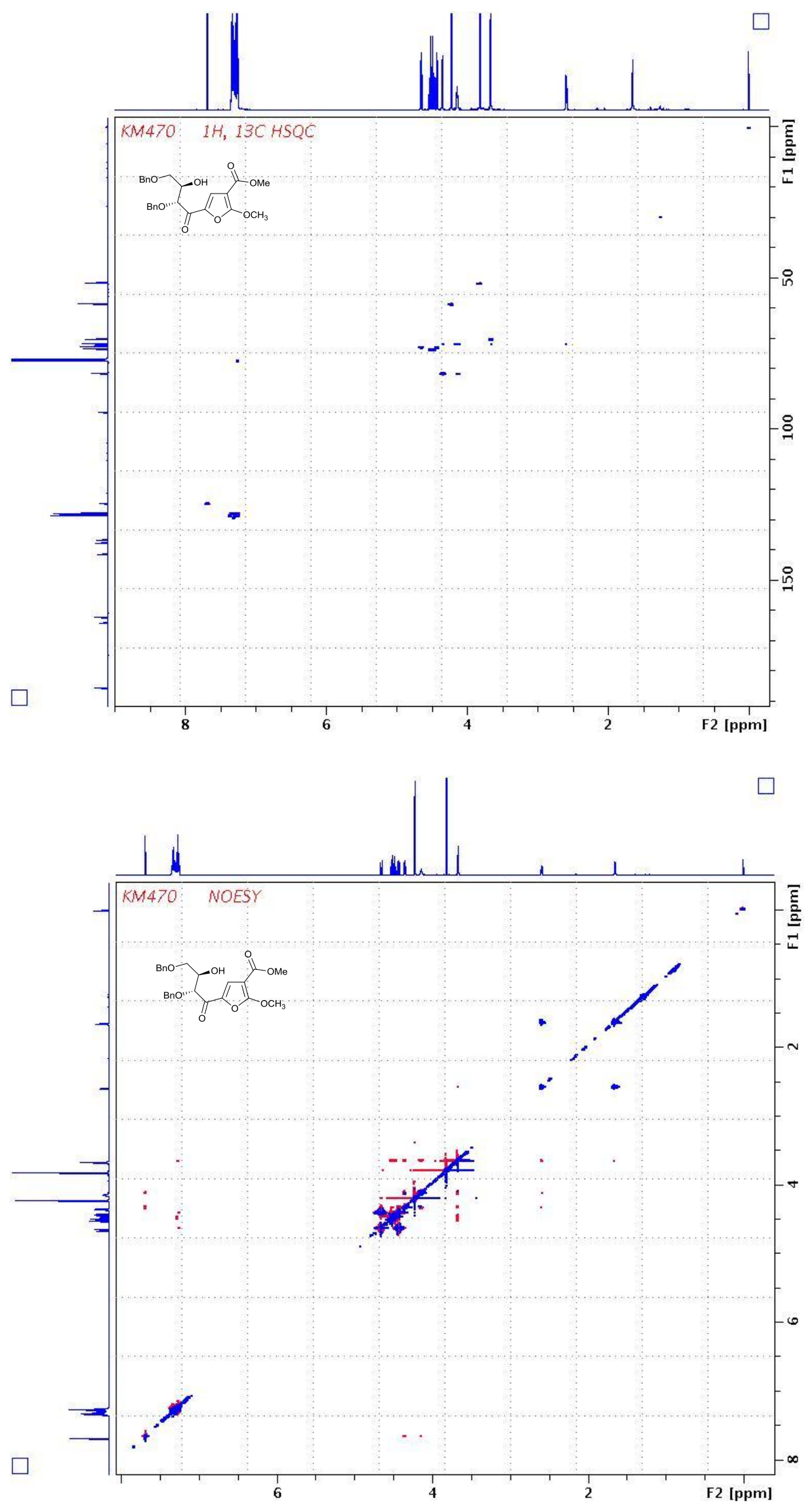


\section{${ }^{1} \mathrm{H},{ }^{13} \mathrm{C}$, DEPT-135 NMR spectra of 3s:}

ID-0915.021.001.1r.esp_KM-490-1H.esp
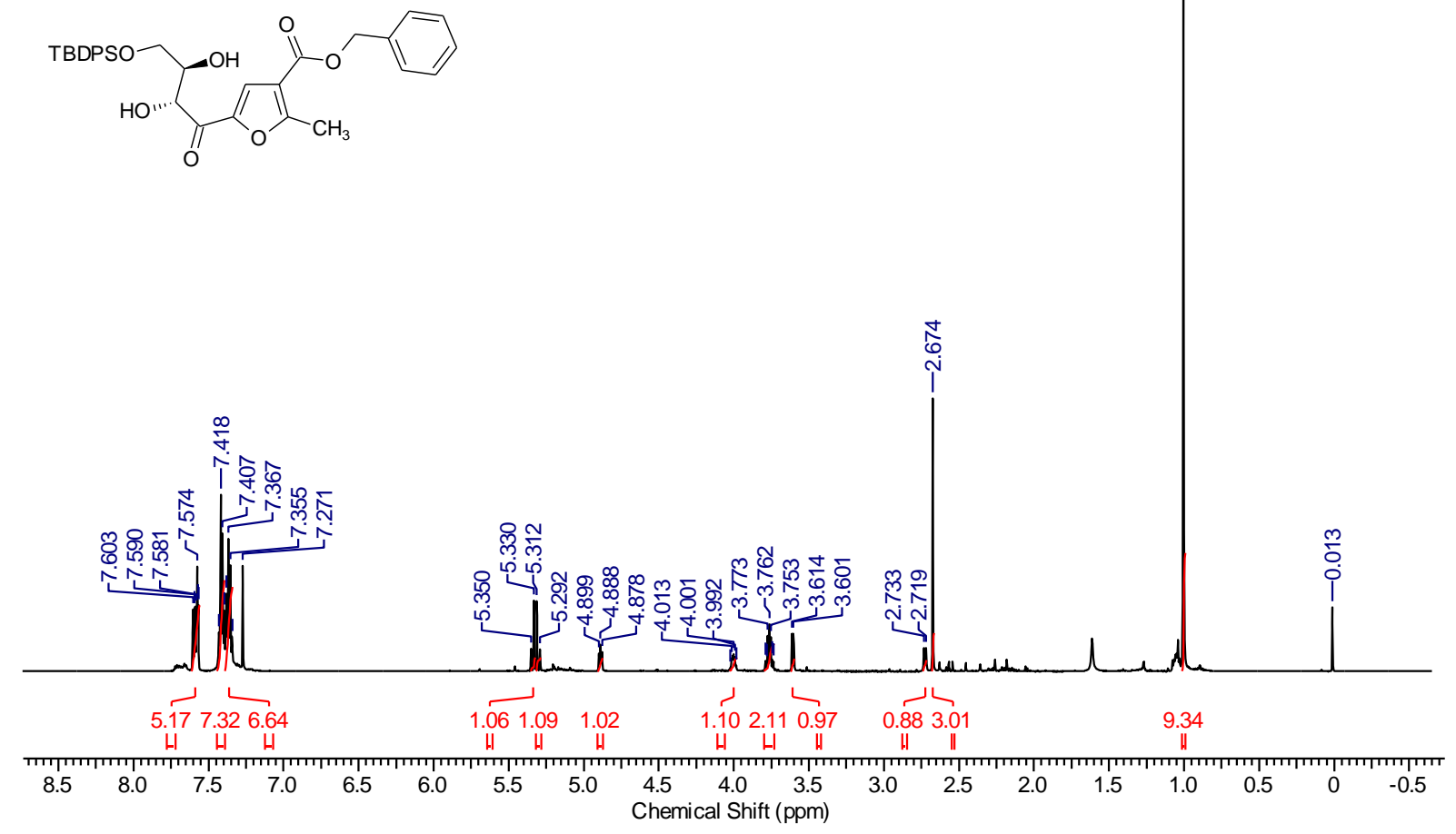

ID-0915.022.001.1r.esp_KM-490-13C.esp
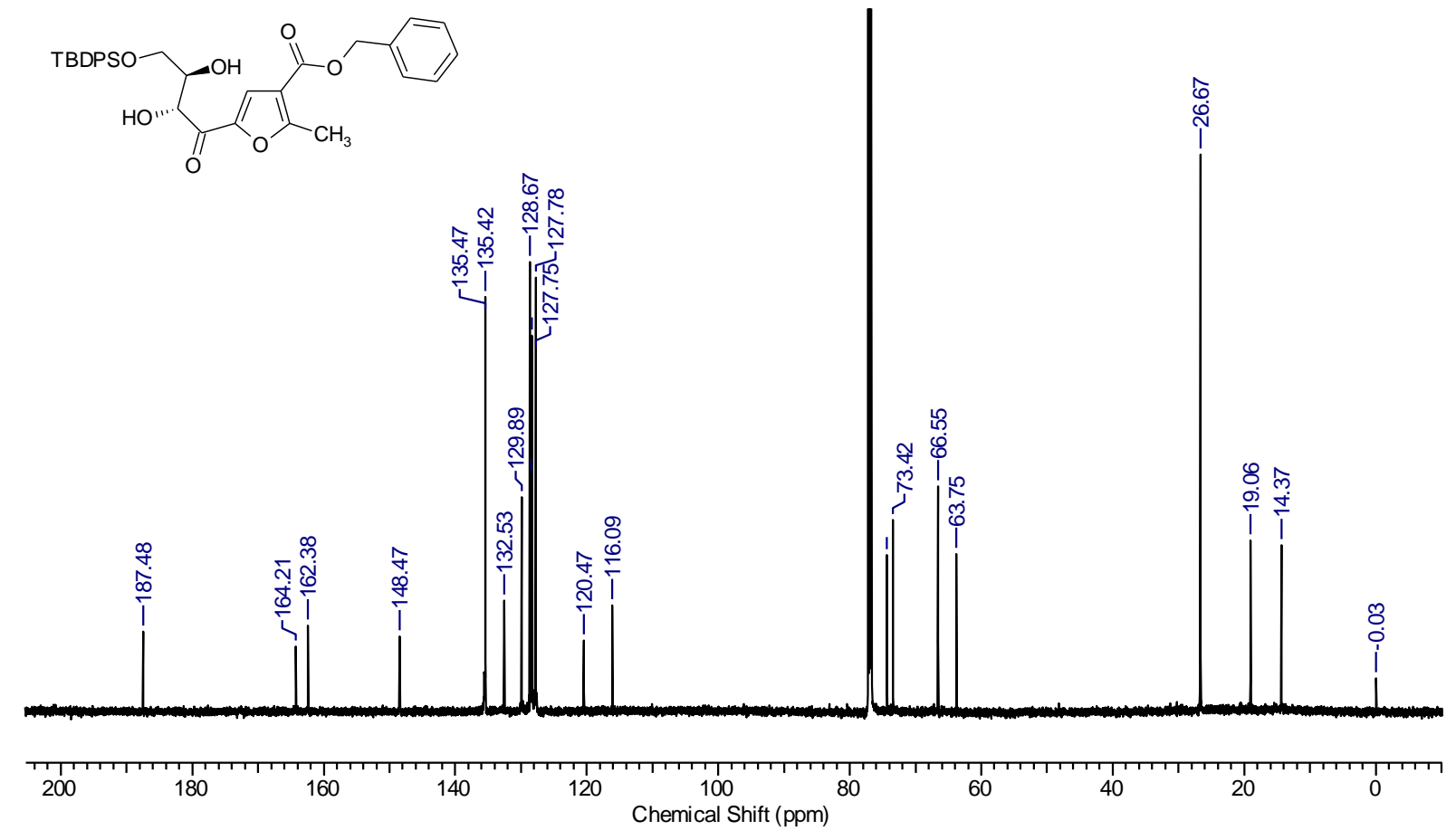


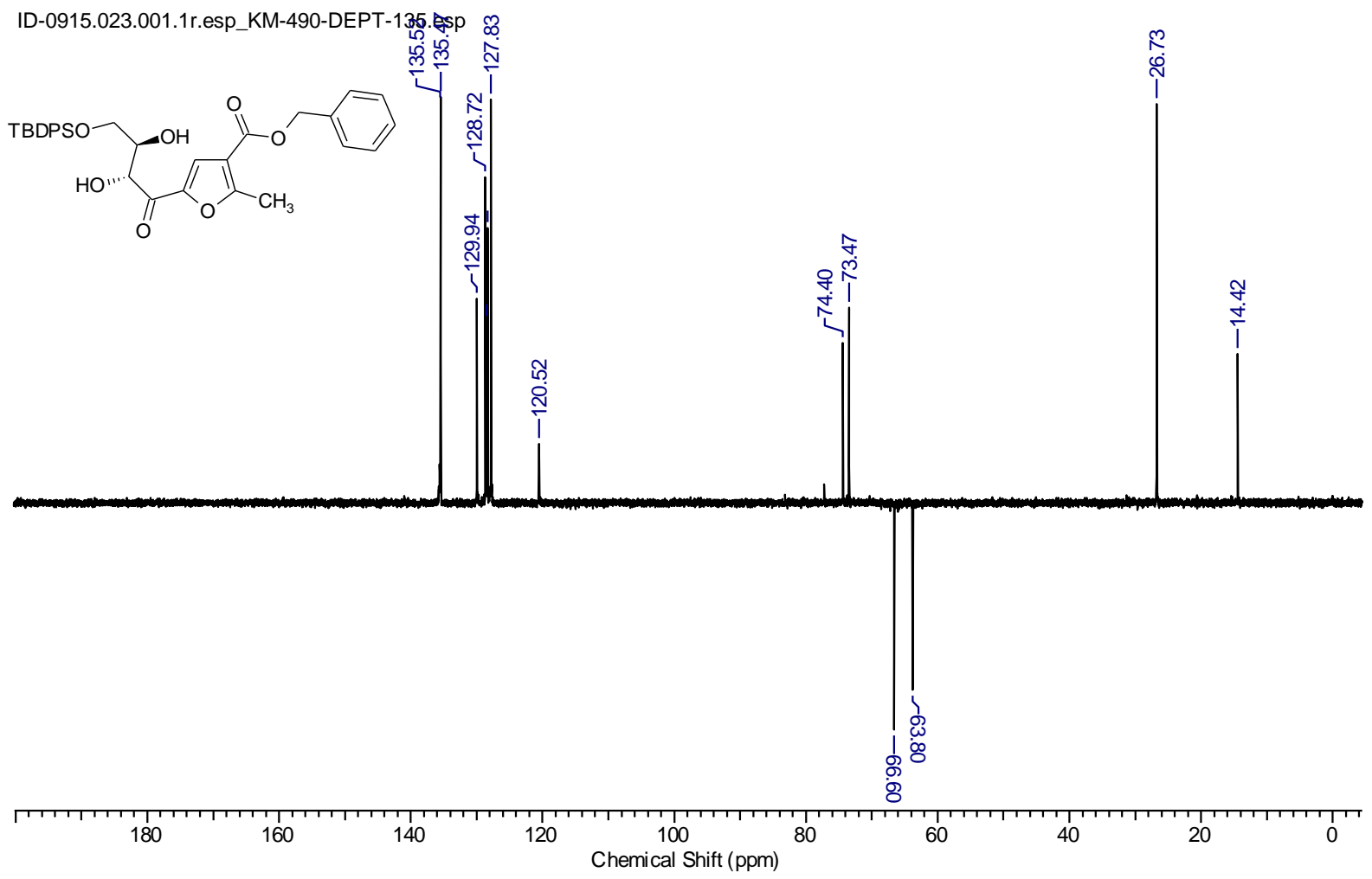

${ }^{1} \mathrm{H},{ }^{13} \mathrm{C}$, DEPT-135 NMR spectra of $3 \mathrm{t}$ and $4 \mathrm{t}$ :

Furan NMR-File.045.001.1r.esp_KM-474-1H.esp<smiles>CC1(C)CC(=O)c2c(C(=O)[C@@H](O)[C@H](O)COCc3ccccc3)coc2C1</smiles>

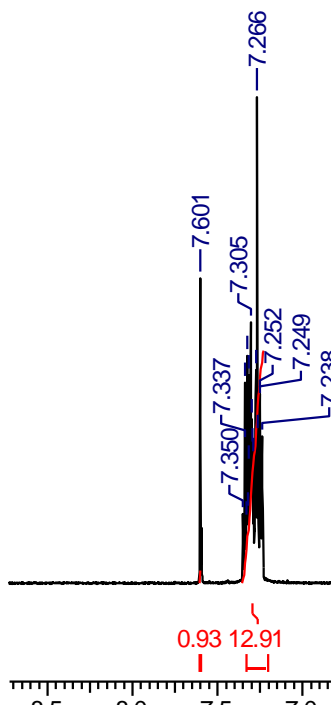

$86: 14$

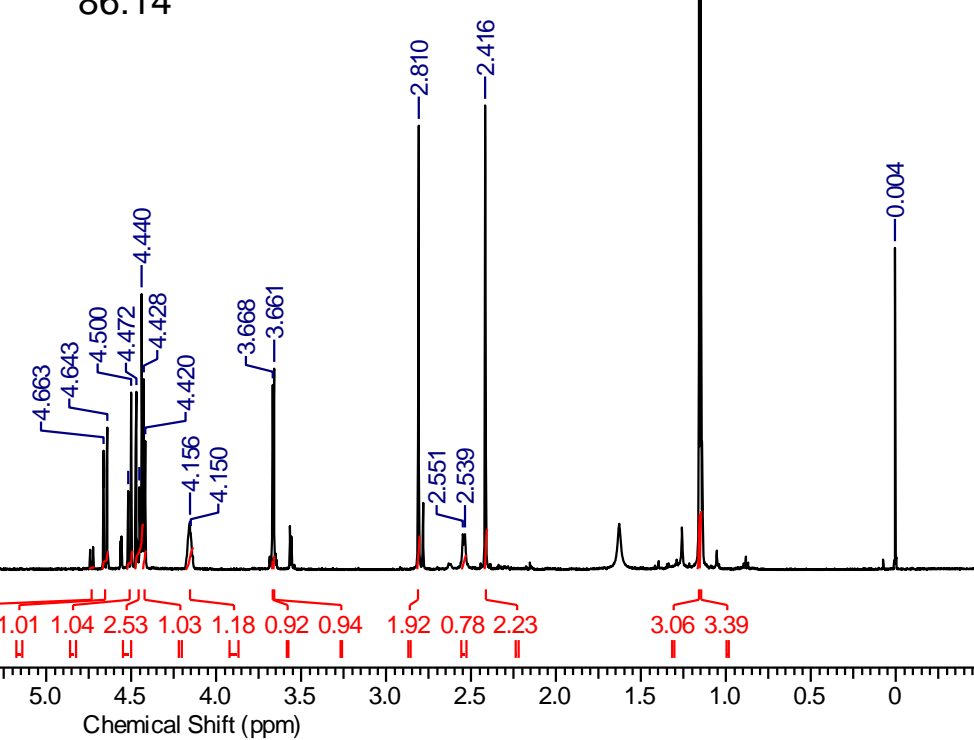



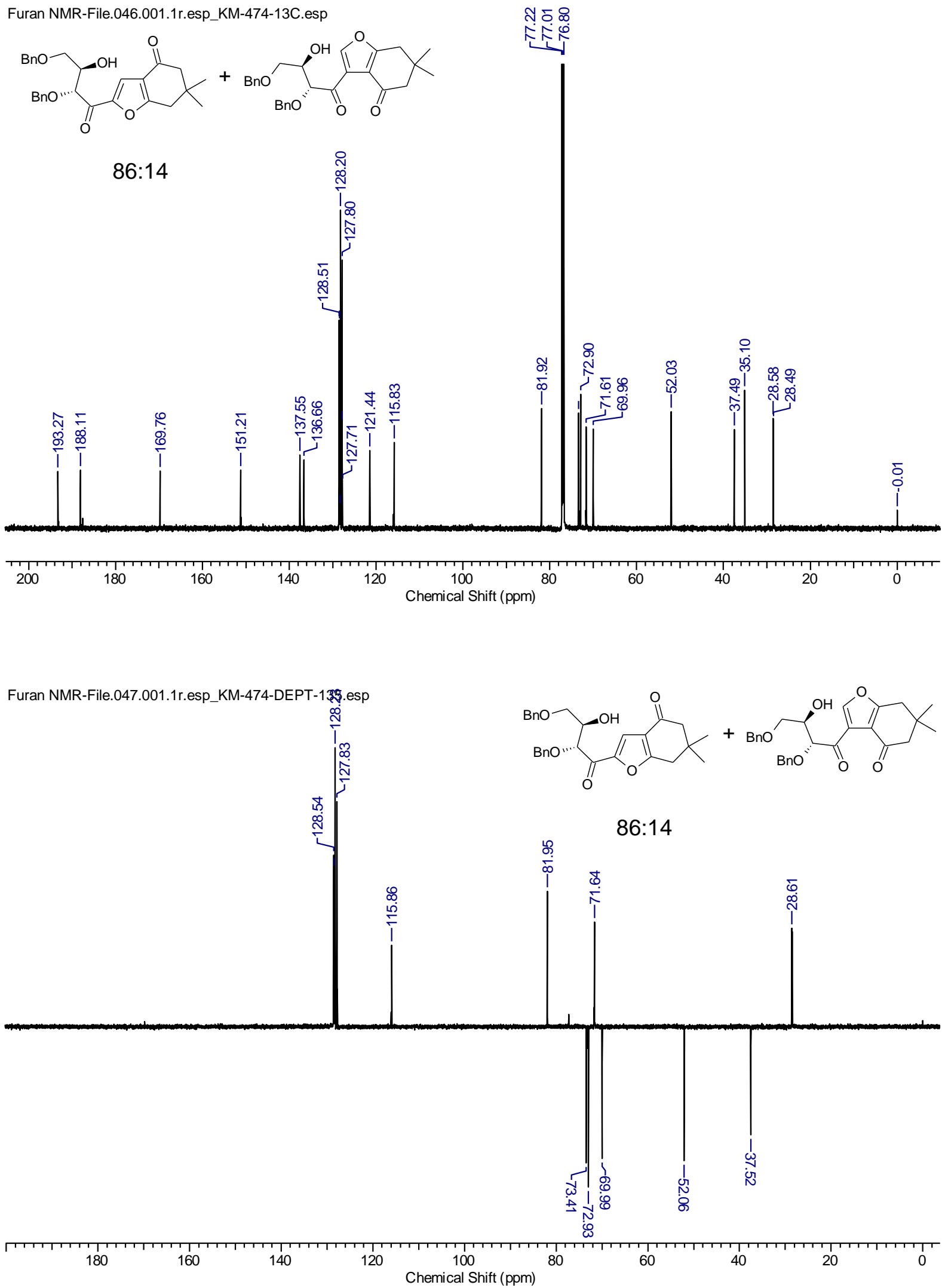


\section{${ }^{1} \mathrm{H},{ }^{13} \mathrm{C}$, DEPT-90 NMR spectra of $3 \mathrm{u}$ and $4 \mathrm{u}$ :}

KM-496-1H.001.001.1r.esp

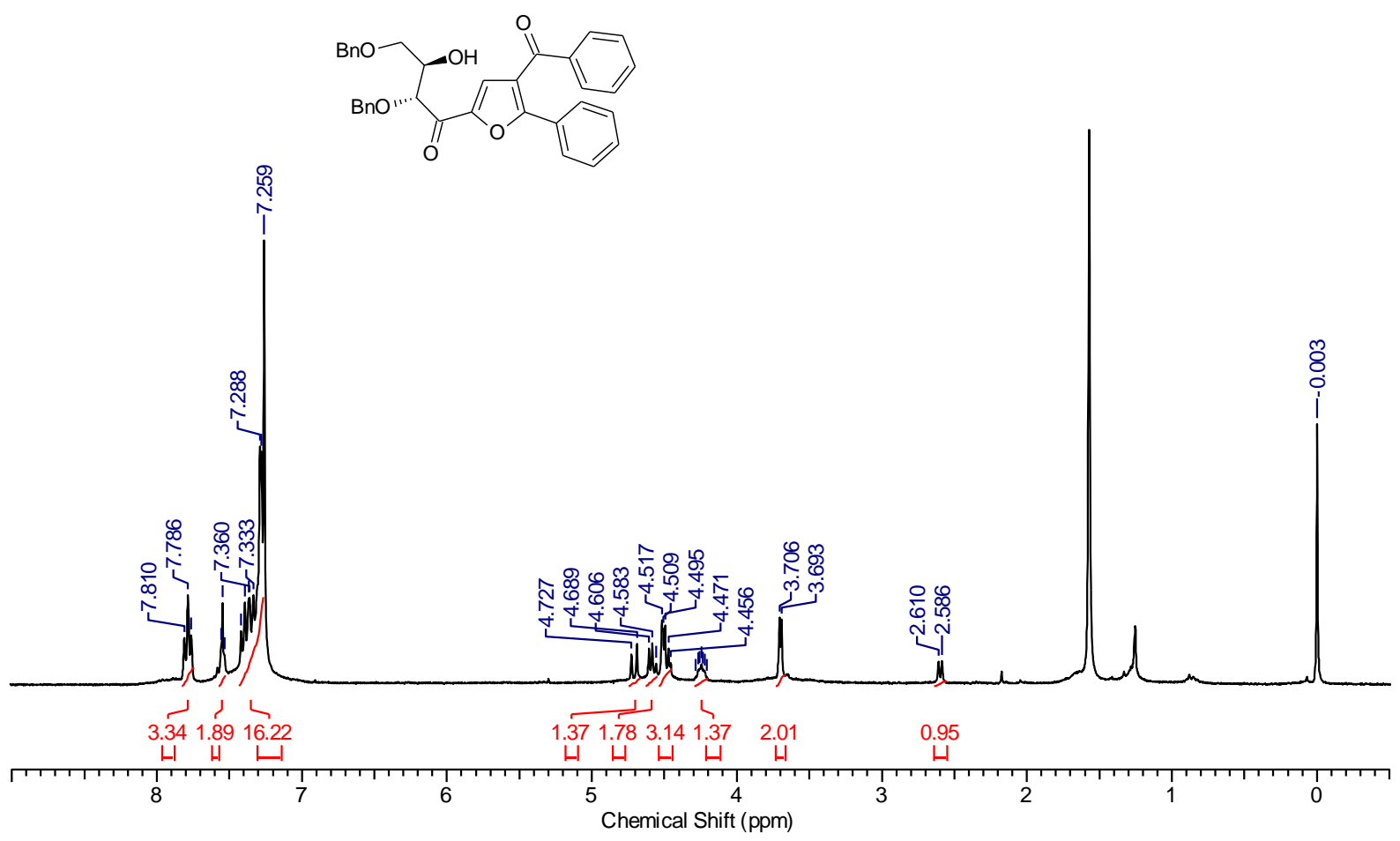

KM-496A-C.001.001.1r.esp
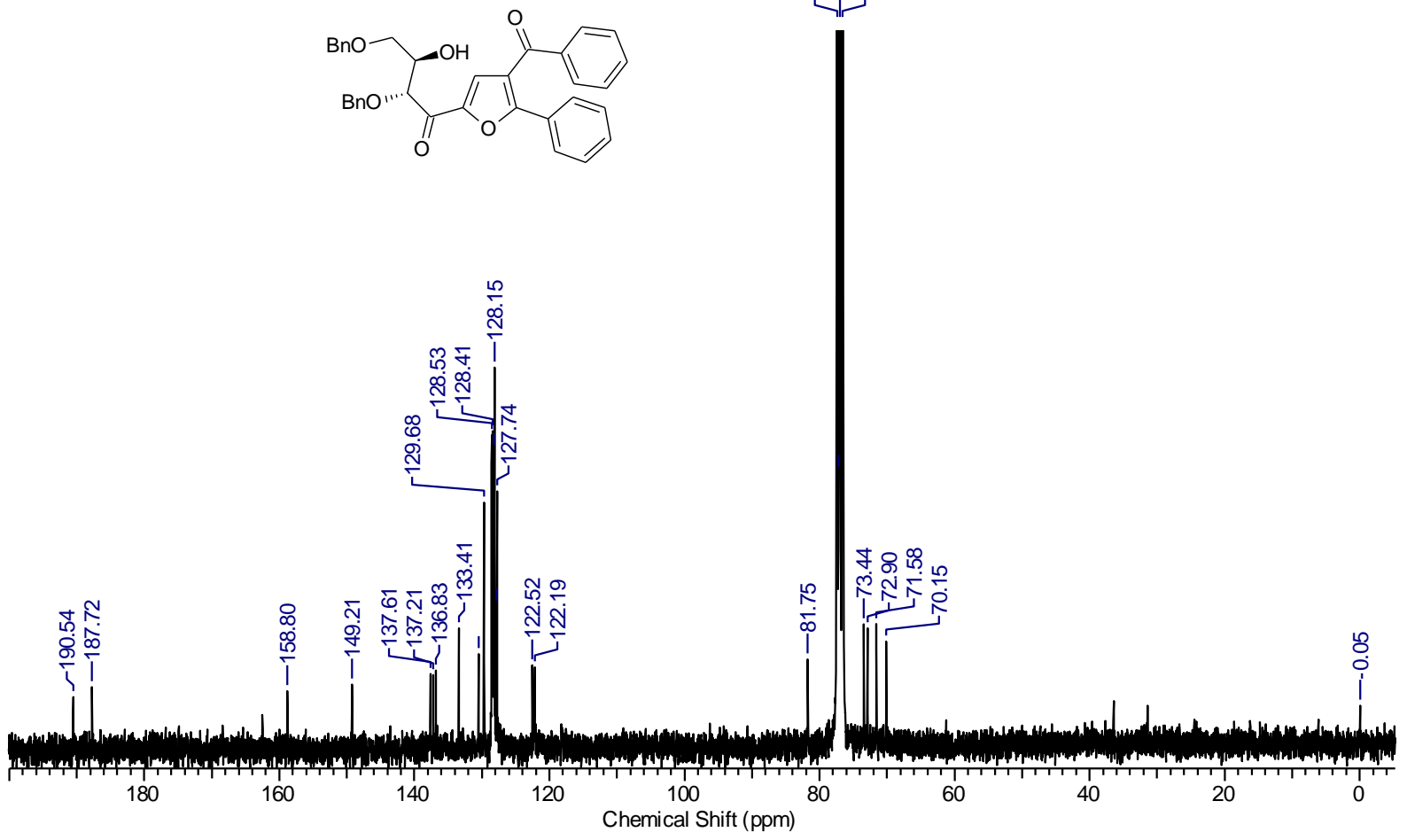


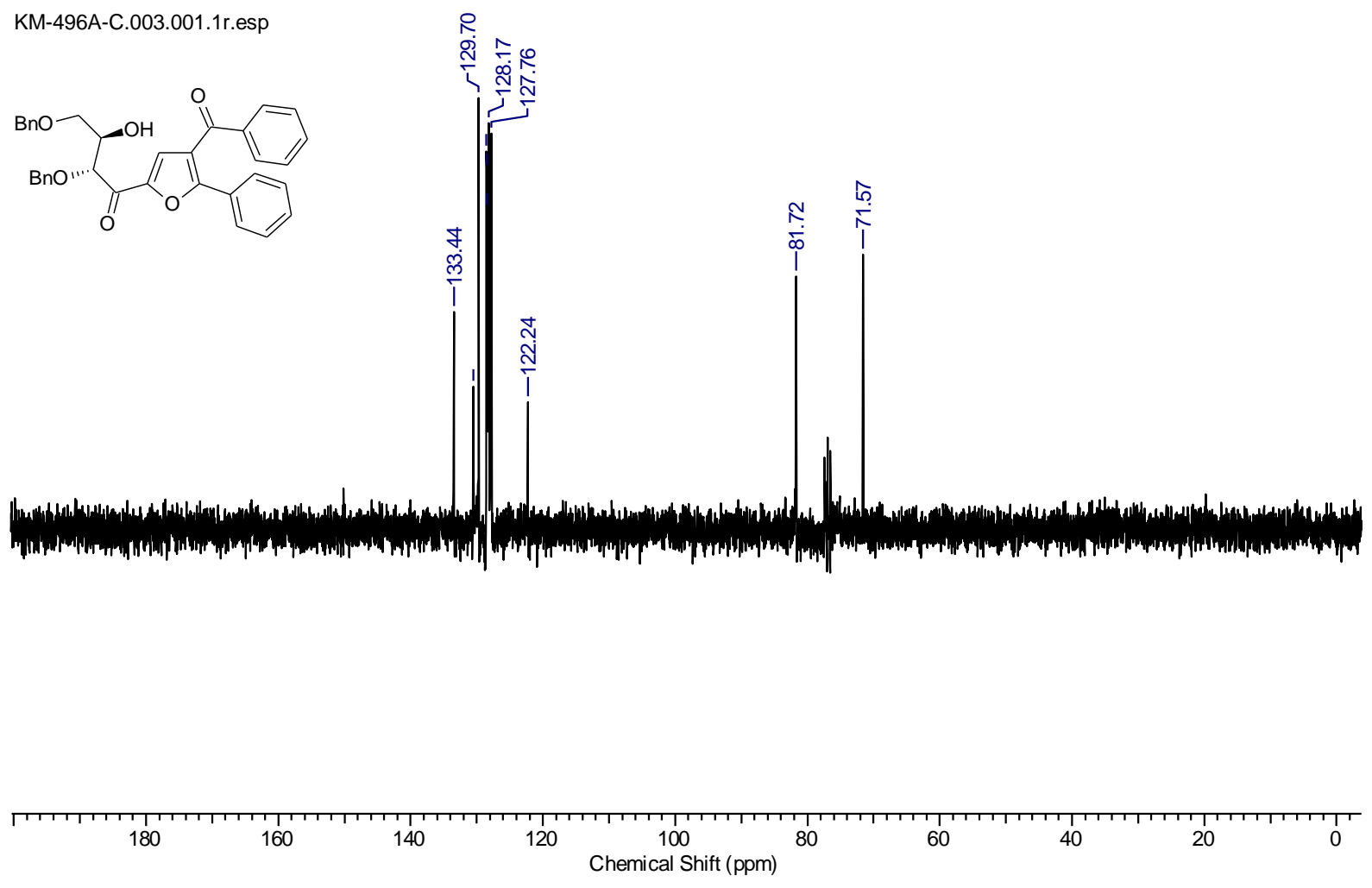

${ }^{1} \mathrm{H},{ }^{13} \mathrm{C}$, DEPT-135 NMR spectra of $3 \mathrm{v}$ and $4 \mathrm{v}$ : 

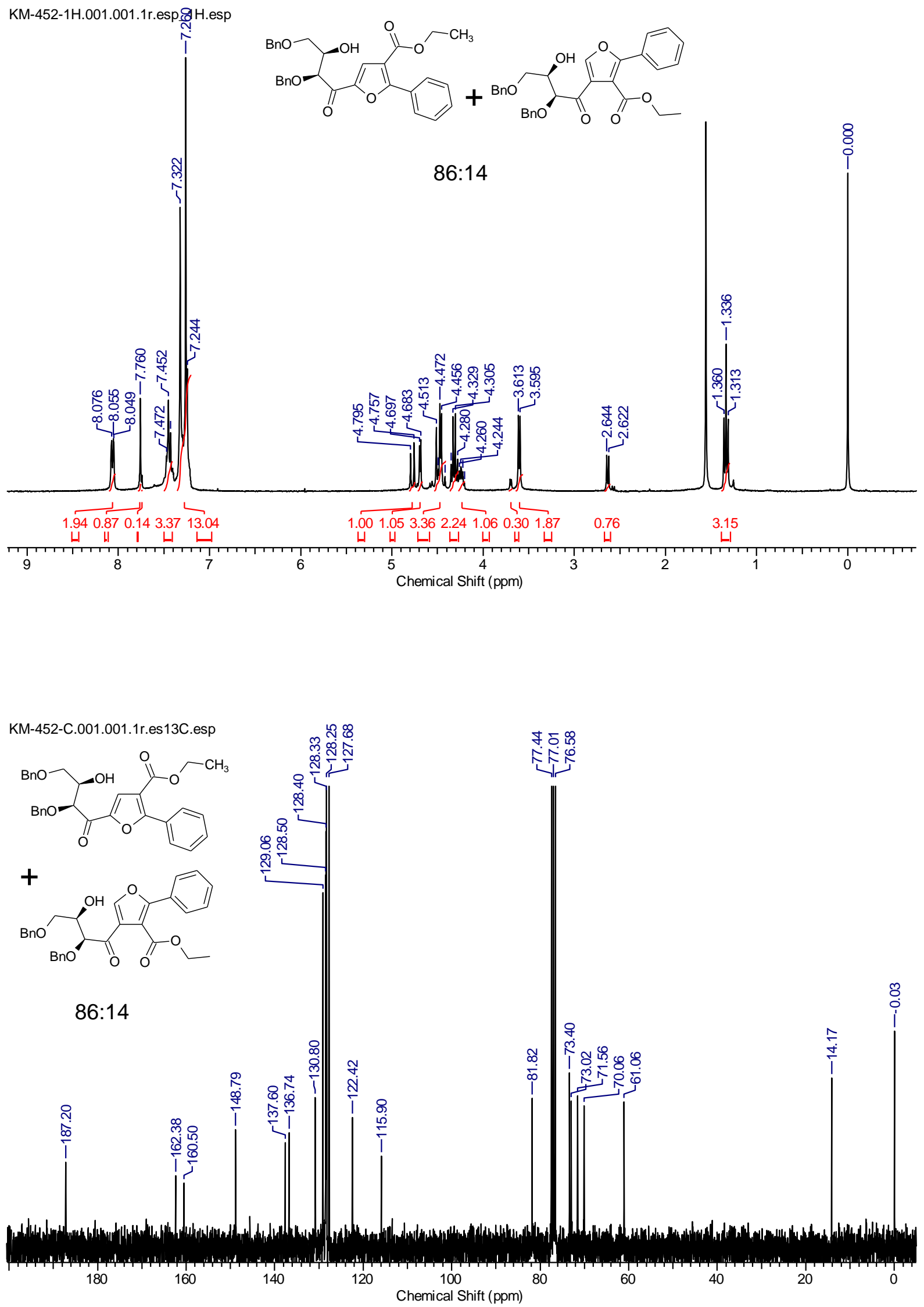


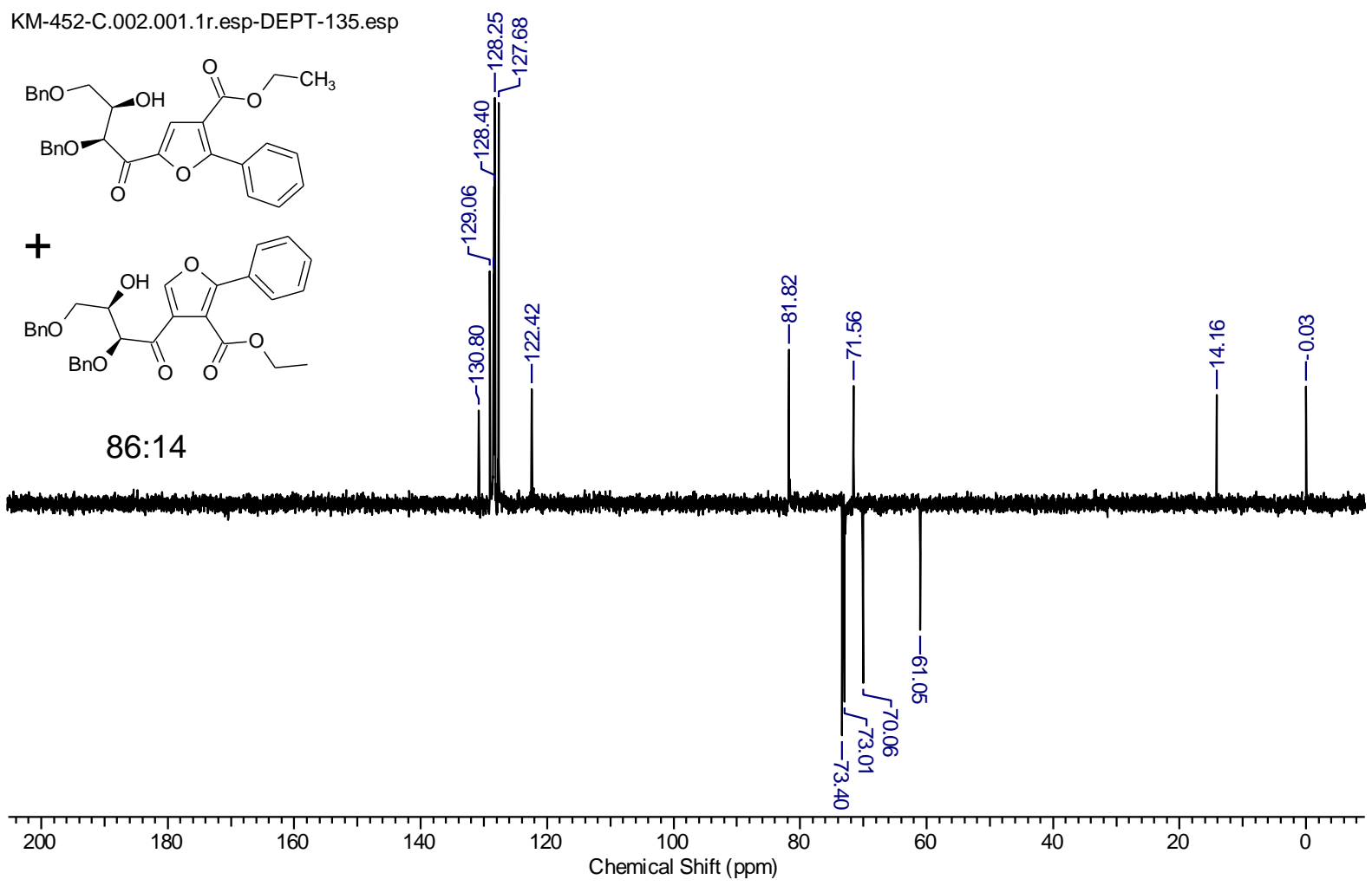

${ }^{1} \mathrm{H},{ }^{13} \mathrm{C}$, DEPT-135 NMR spectra of 3w:

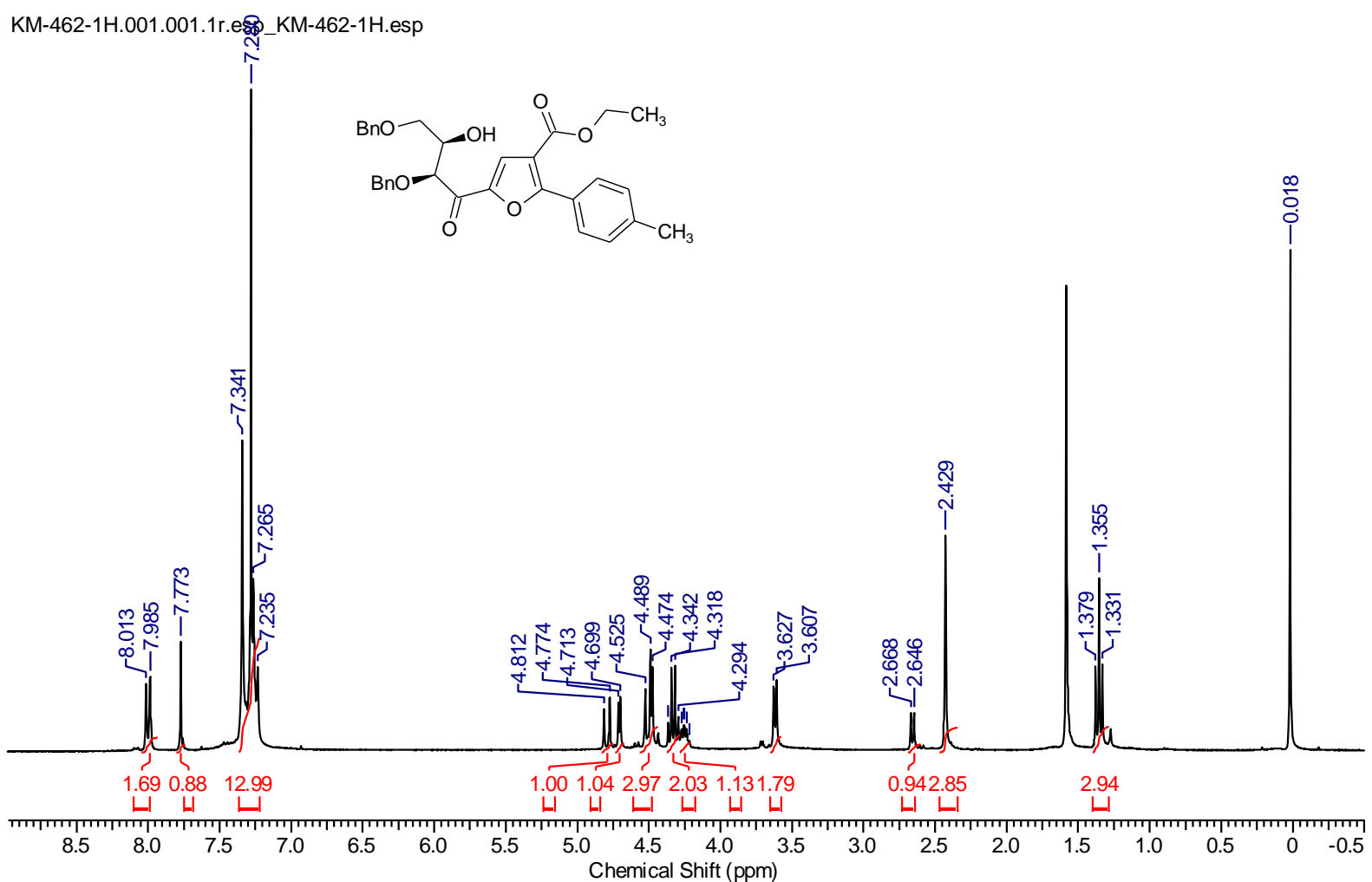



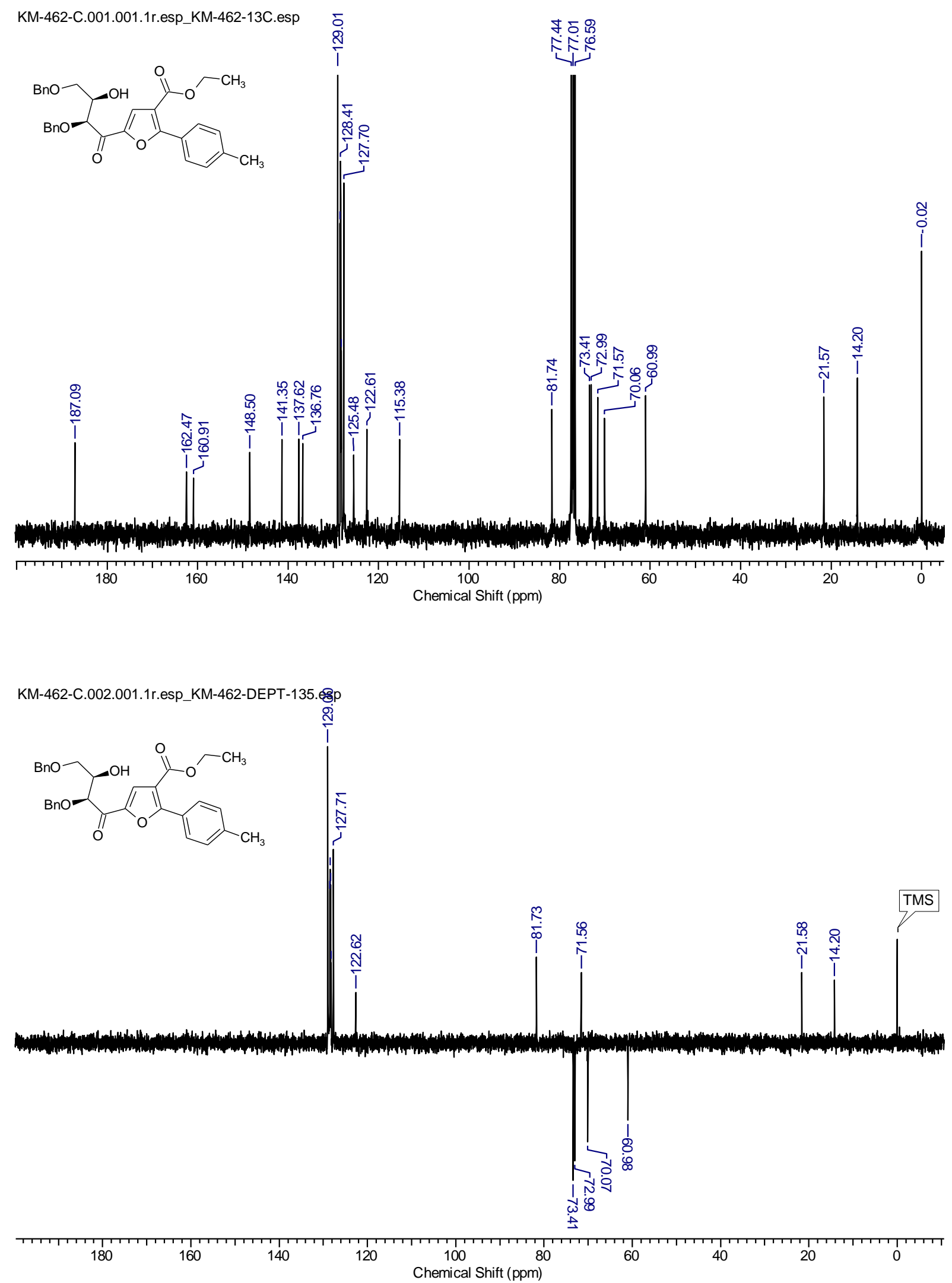
${ }^{1} \mathrm{H},{ }^{13} \mathrm{C}$, DEPT-135 NMR spectra of $3 \mathrm{x}$ and $4 \mathrm{x}$ :
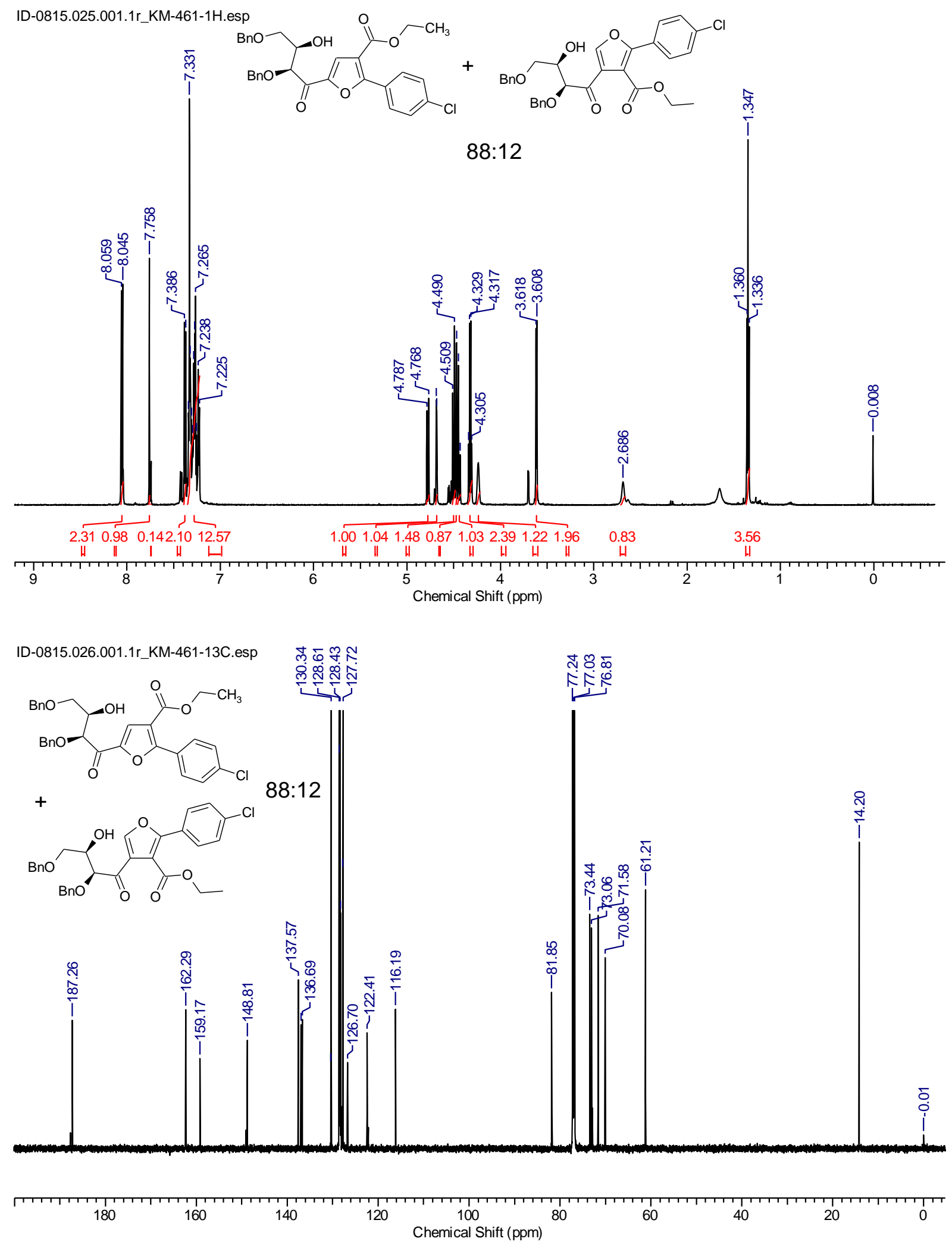


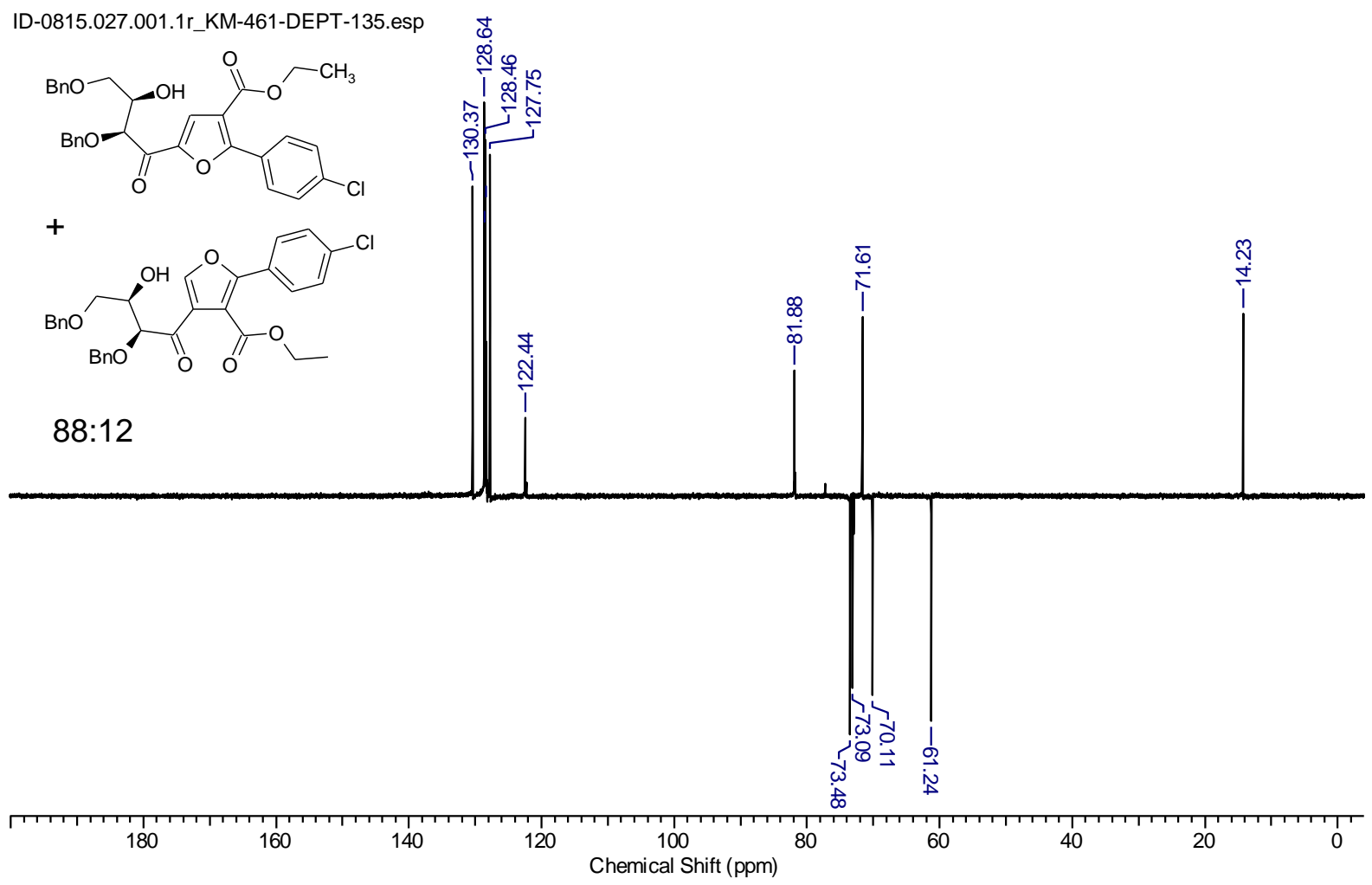

\section{${ }^{1} \mathrm{H},{ }^{13} \mathrm{C}$, DEPT-135 NMR spectra of 3y:}

ID-0815.091.001.1r.esp_KM-467-1H_600.esp<smiles>COC(=O)c1cc(C(=O)C(O)C(O)C(O)COc2ccccc2)oc1OC</smiles>
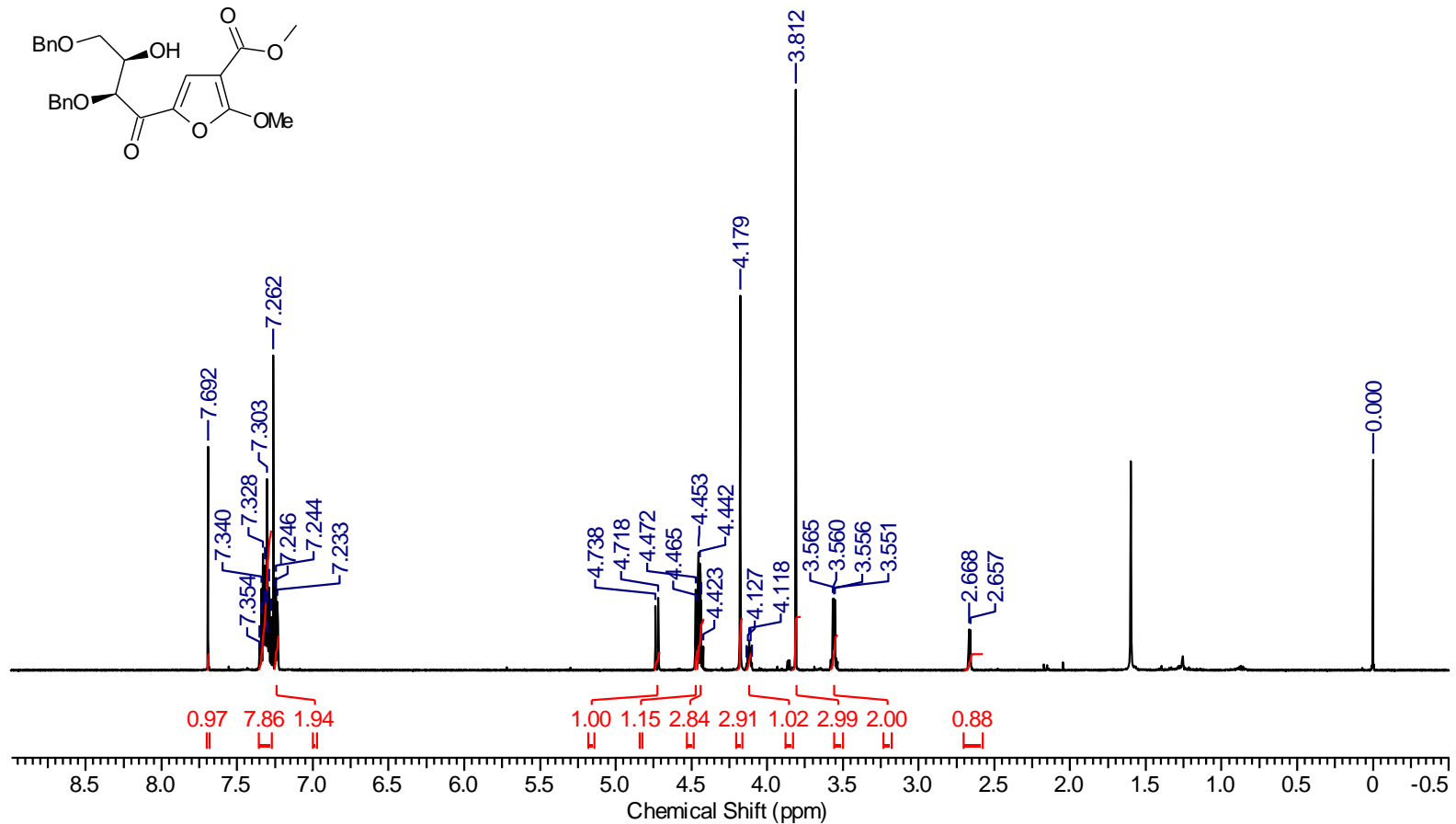

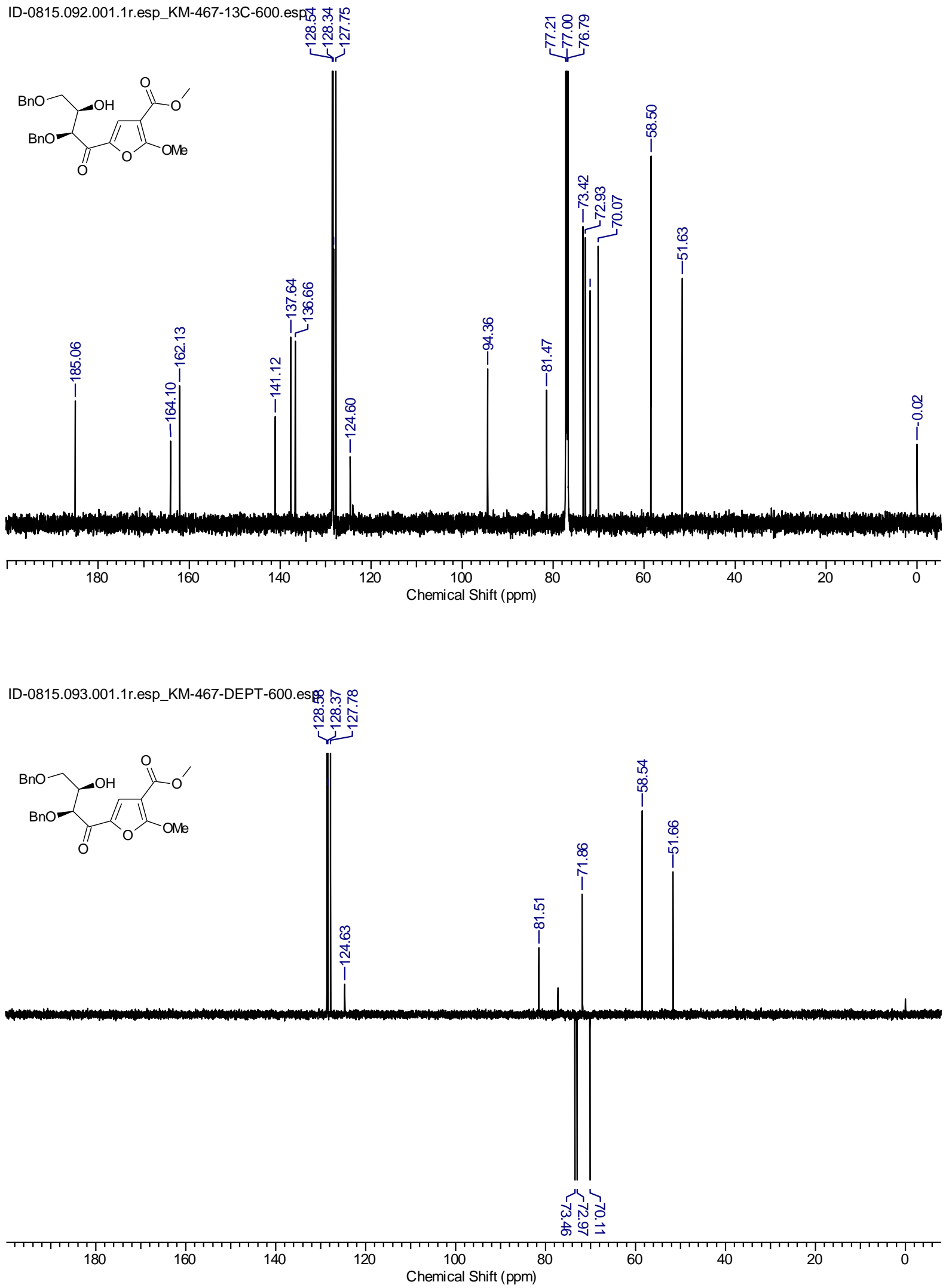
2D (COSY, HMBC, HSQC, and NOESY) NMR spectra of 3y:

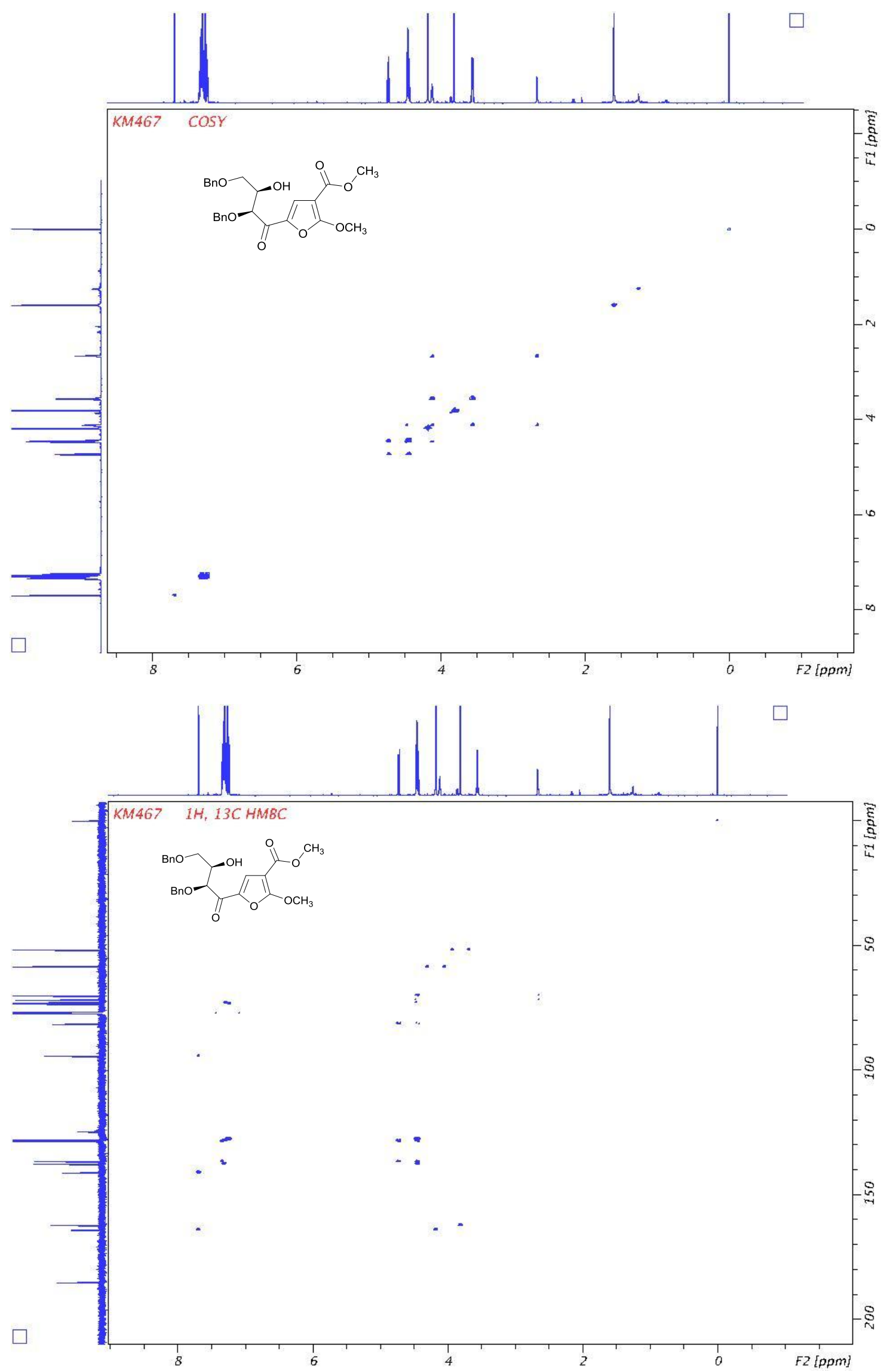

S110 

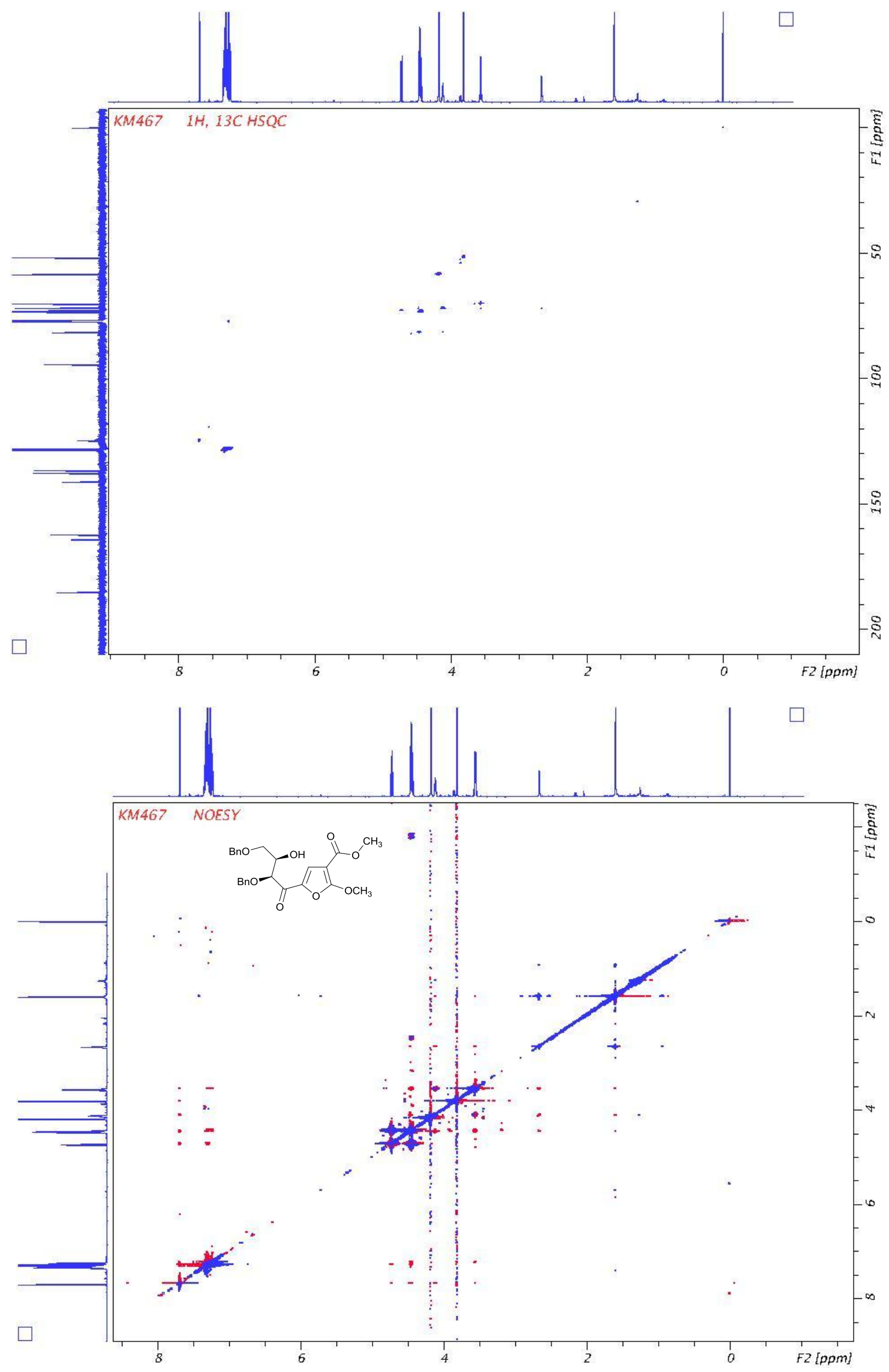
${ }^{1} \mathrm{H},{ }^{13} \mathrm{C}$, DEPT-135 NMR spectra of 3z:

ID-0915.029.001.1r.esp_KM-492-1H.esp<smiles>Cc1oc(C(=O)[C@H]([18OH])CO)cc1C(=O)OCc1ccccc1</smiles>
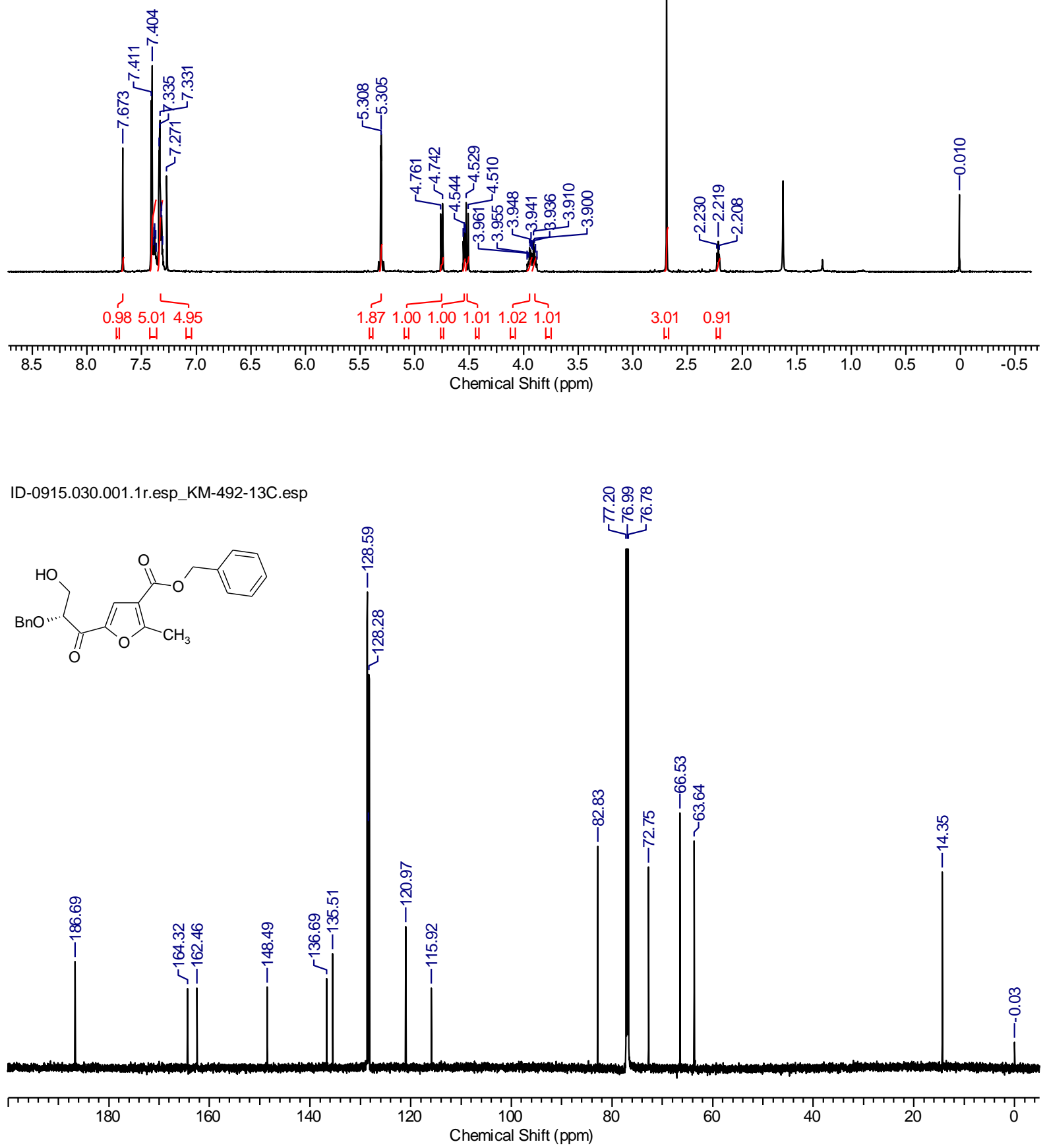


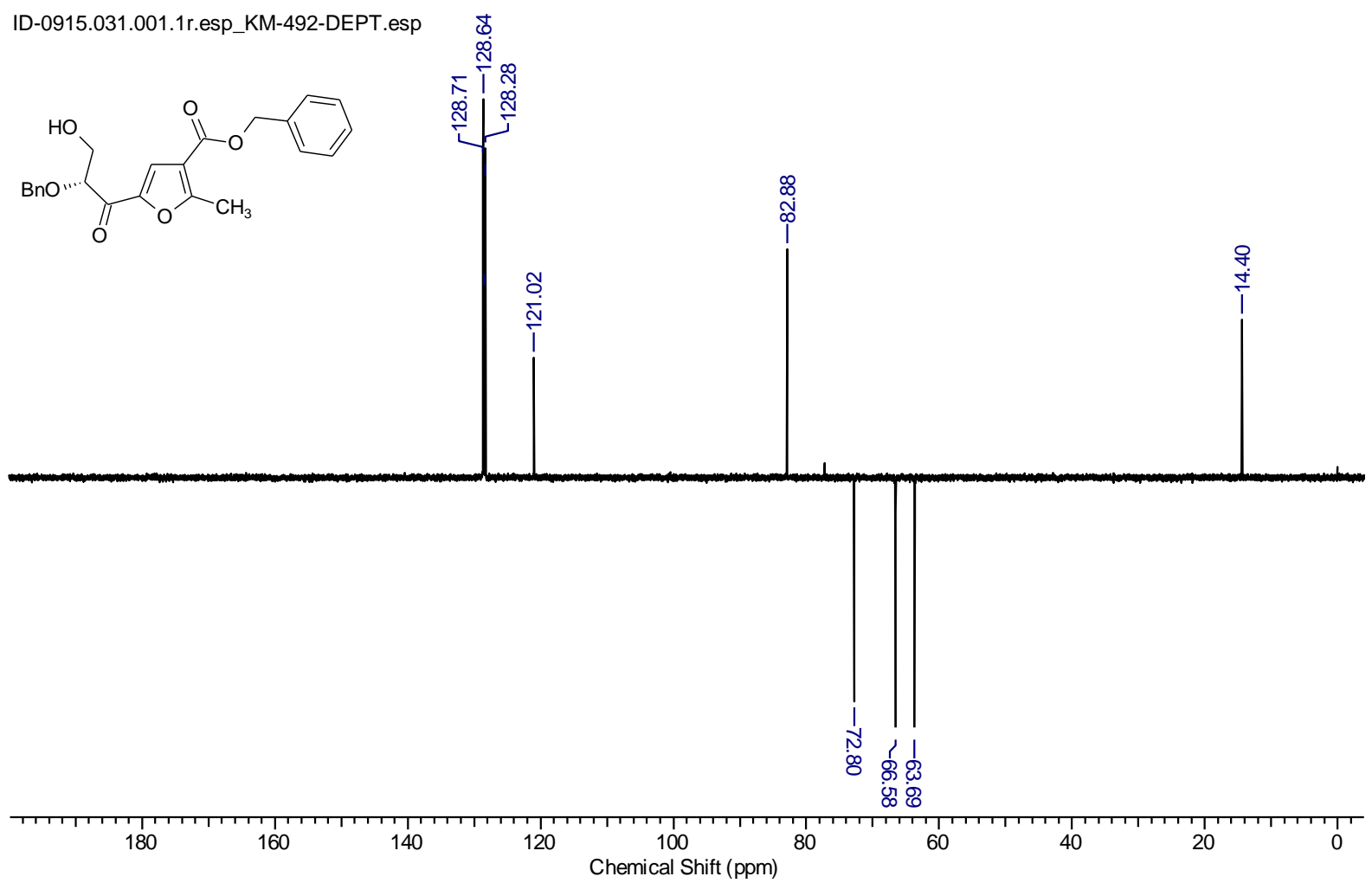

${ }^{1} \mathrm{H},{ }^{13} \mathrm{C}$, DEPT-135 NMR spectra of 5a:

KM-494-1H.esp<smiles>C=C(OCc1ccccc1)C(=O)c1cc(C(=O)OCc2ccccc2)c(C)o1</smiles>
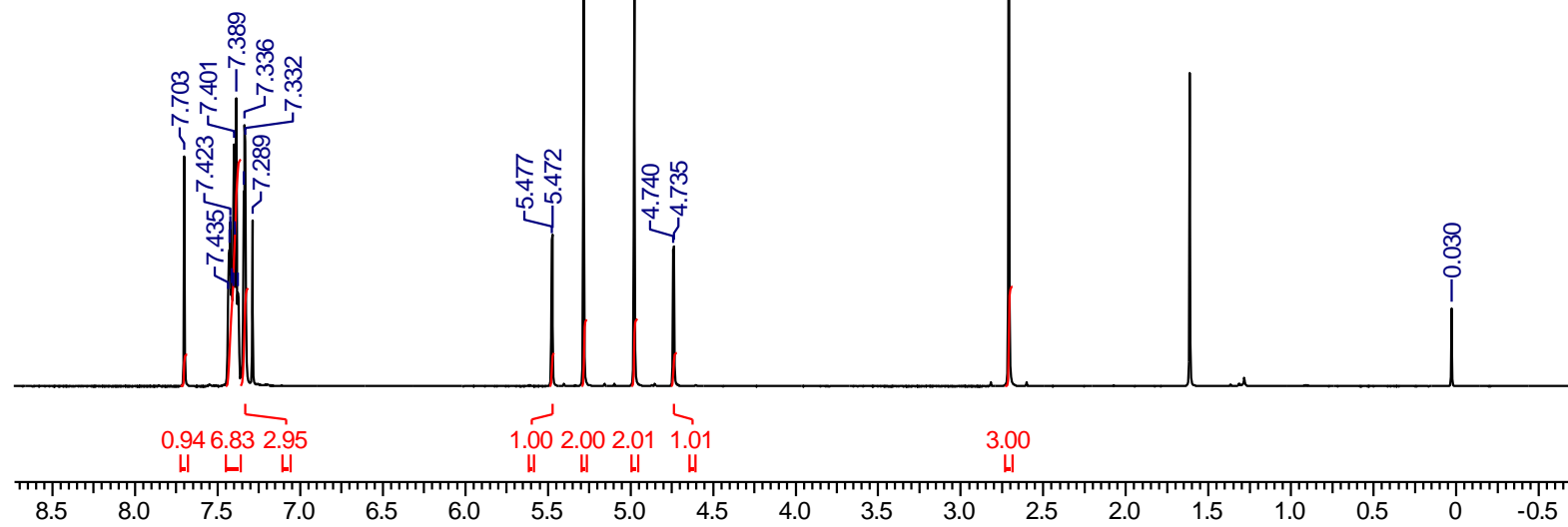

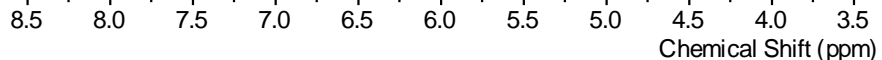



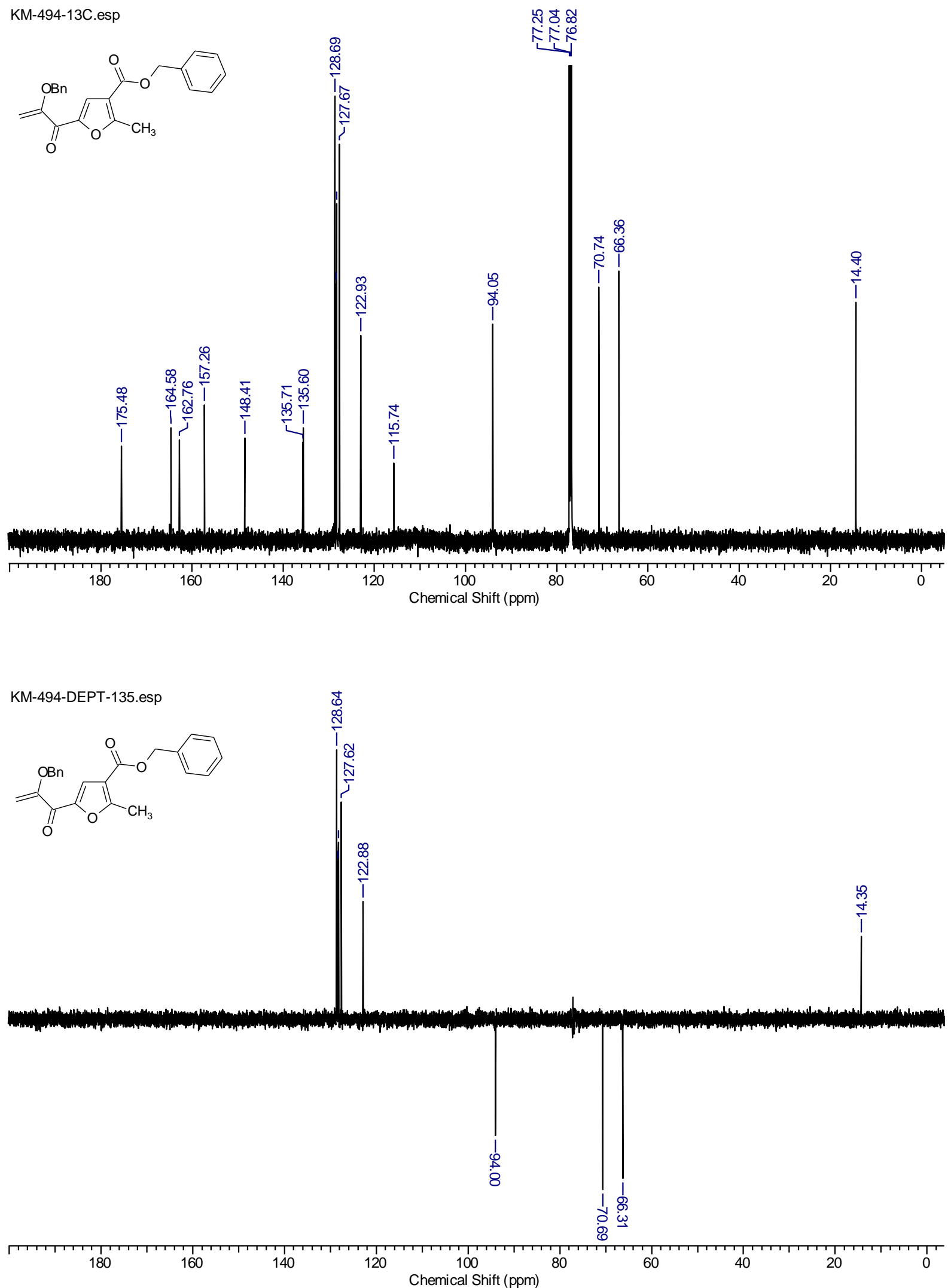
2D (COSY, HMBC, HSQC, and NOESY) NMR spectra of 5a:
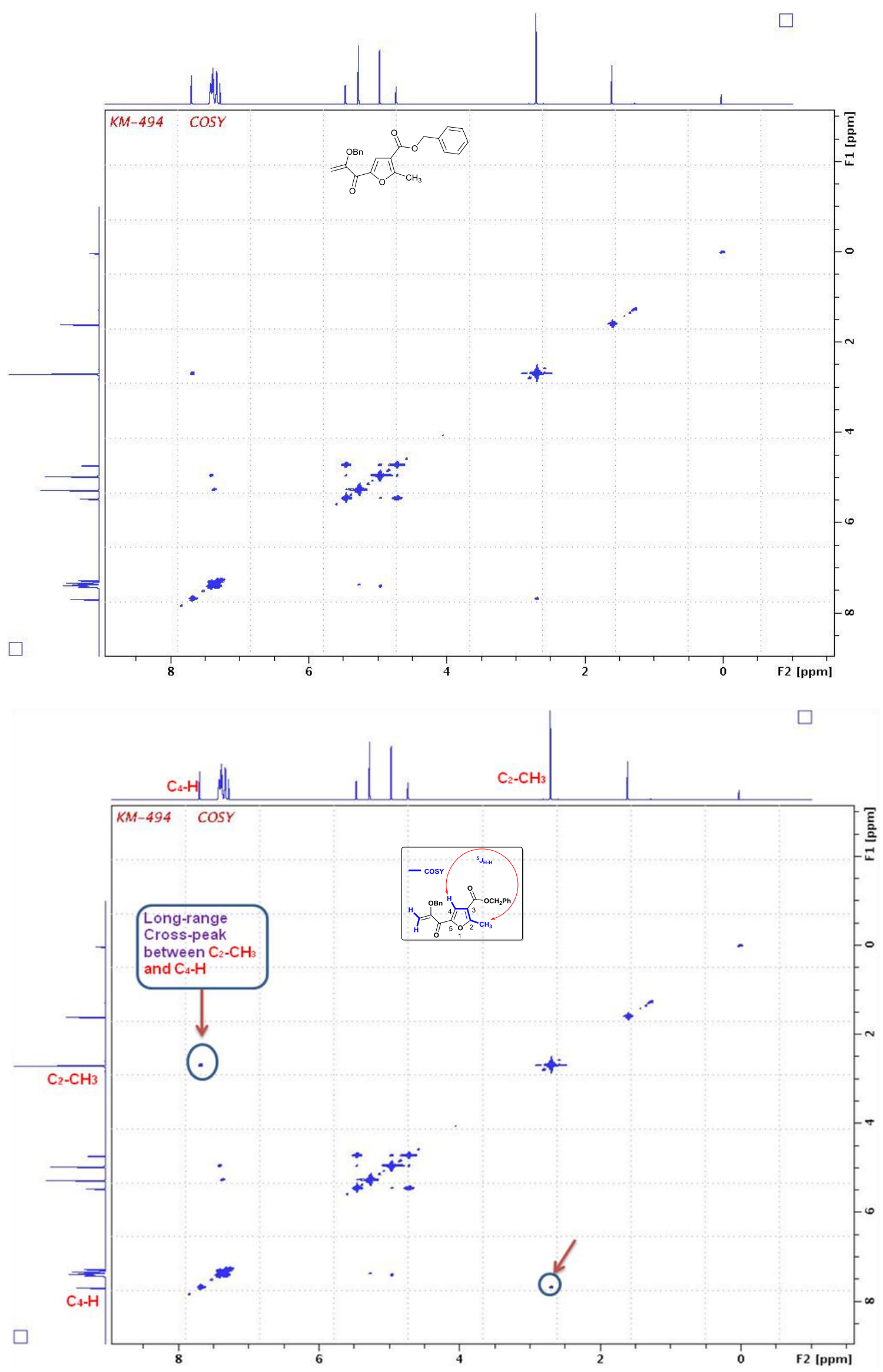

S115 

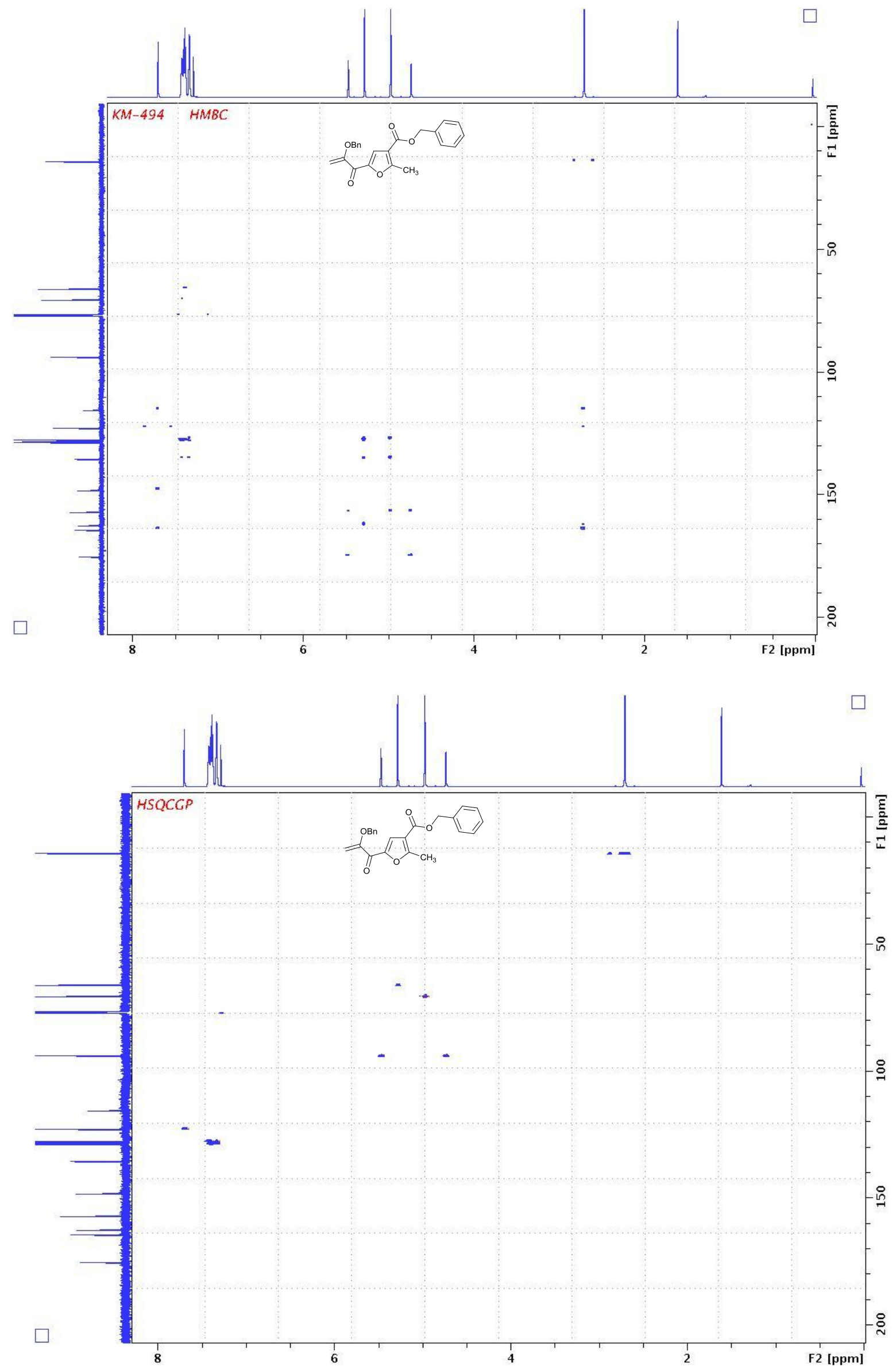


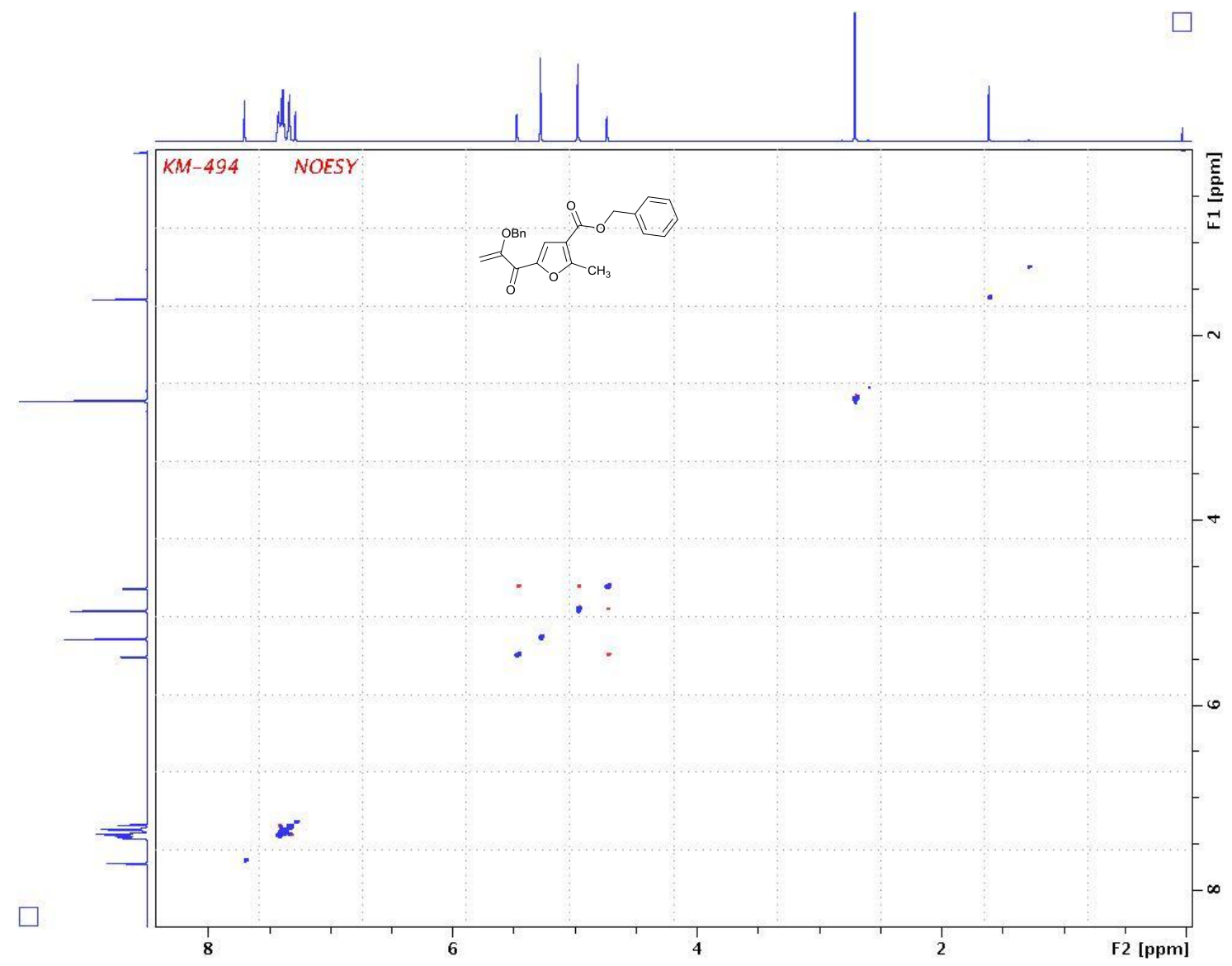

${ }^{1} \mathrm{H},{ }^{13} \mathrm{C}$, DEPT-135 NMR spectra of 5b:

KM-497-1H.001.001.1r.esp_KM-497-1H.esp

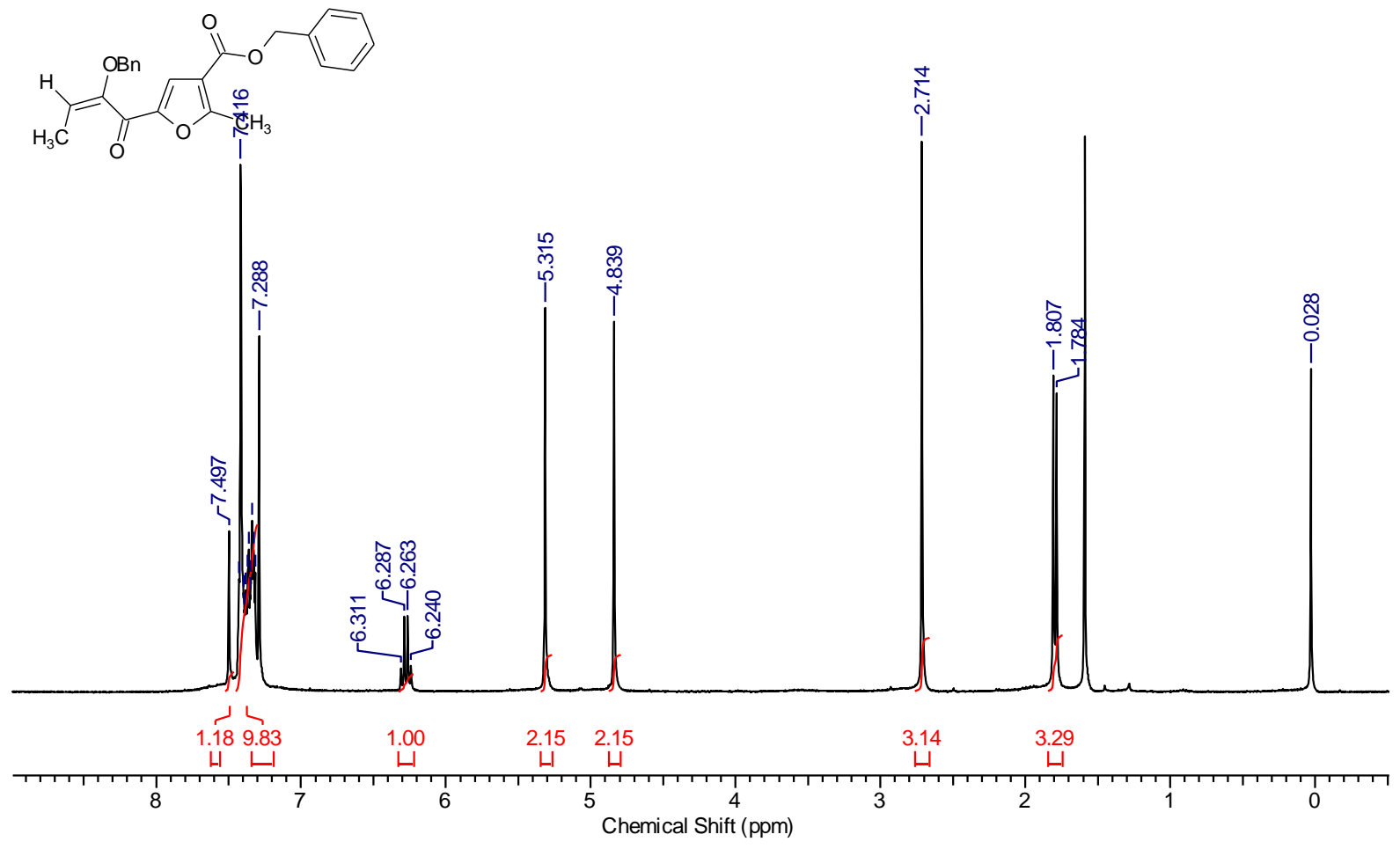



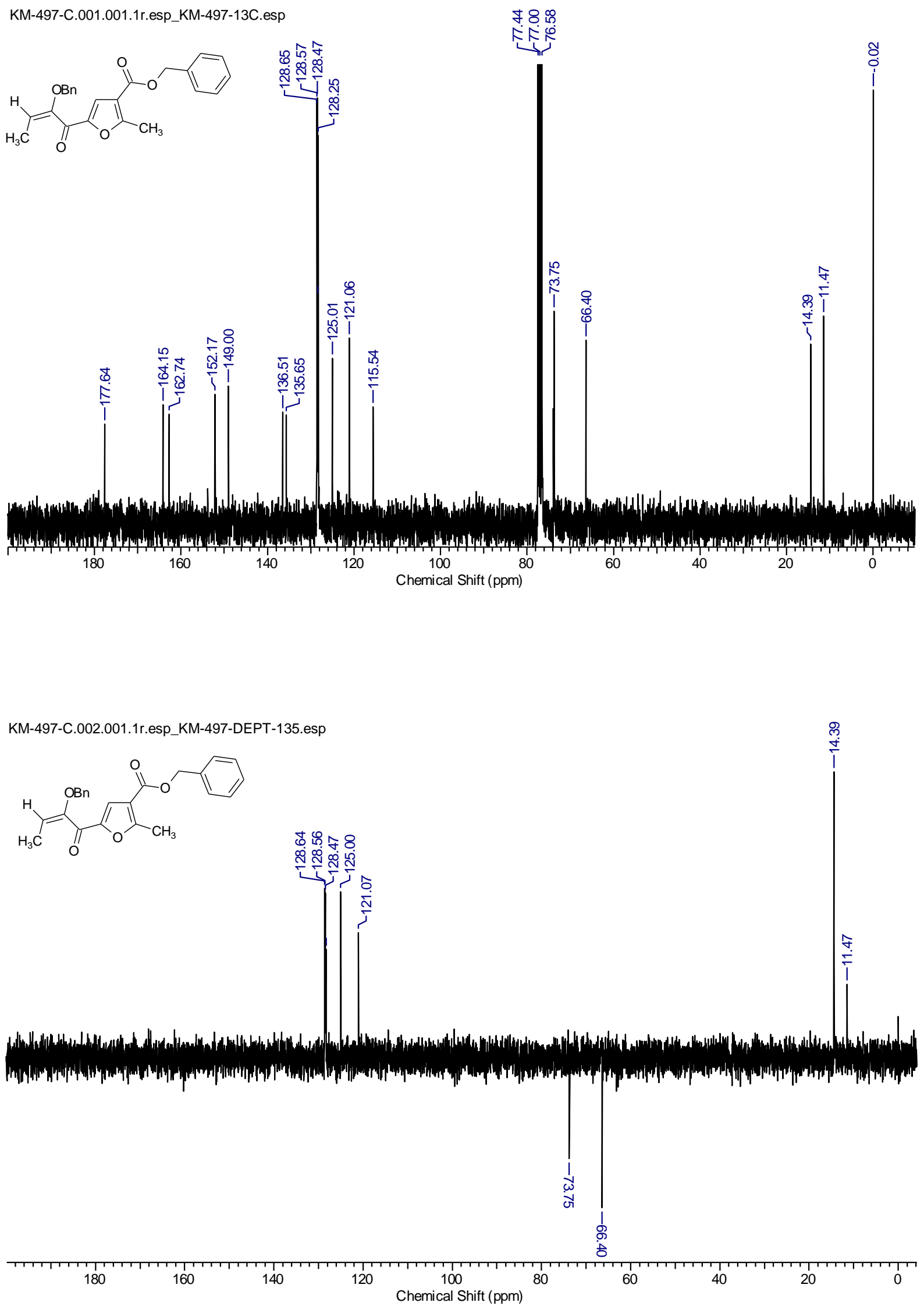